University of Louisville

ThinkIR: The University of Louisville's Institutional Repository

Electronic Theses and Dissertations

$12-2018$

\title{
Effects of hydrogen sulfide in hyperhomocysteinemia-mediated skeletal muscle myopathy.
}

Avisek Majumder

University of Louisville

Follow this and additional works at: https://ir.library.louisville.edu/etd

Part of the Nutritional and Metabolic Diseases Commons

\section{Recommended Citation}

Majumder, Avisek, "Effects of hydrogen sulfide in hyperhomocysteinemia-mediated skeletal muscle myopathy." (2018). Electronic Theses and Dissertations. Paper 3114.

https://doi.org/10.18297/etd/3114

This Doctoral Dissertation is brought to you for free and open access by ThinkIR: The University of Louisville's Institutional Repository. It has been accepted for inclusion in Electronic Theses and Dissertations by an authorized administrator of ThinkIR: The University of Louisville's Institutional Repository. This title appears here courtesy of the author, who has retained all other copyrights. For more information, please contact thinkir@louisville.edu. 


\title{
EFFECTS OF HYDROGEN SULFIDE IN HYPERHOMOCYSTEINEMIA- MEDIATED SKELETAL MUSCLE MYOPATHY
}

\author{
By
}

Avisek Majumder

B. Sc. Vidyasagar University, India, 2008

M.Sc. West Bengal University of Technology, India, 2010

M.S. University of Louisville, USA, 2017

\author{
A Dissertation \\ Submitted to the Faculty of the \\ School of Medicine of the University of Louisville \\ in Partial Fulfillment of the Requirements \\ For the Degree of
}

Doctor of Philosophy

in Biochemistry and Molecular Genetics

Department of Biochemistry and Molecular Genetics

University of Louisville

Louisville, Kentucky

[

December 2018 
Copyright 2018 by Avisek Majumder

All rights reserved 



\title{
EFFECTS OF HYDROGEN SULFIDE IN HYPERHOMOCYSTEINEMIA- MEDIATED SKELETAL MUSCLE MYOPATHY
}

\author{
By \\ Avisek Majumder \\ B. Sc. Vidyasagar University, India, 2008 \\ M.Sc. West Bengal University of Technology, India, 2010 \\ M.S. University of Louisville, USA, 2017
}

A Dissertation Approved on

November $20^{\text {th }}, 2018$

By the following Dissertation Committee

Dr. Suresh C. Tyagi, Ph.D., Mentor

Dr. Barbara J. Clark, Ph.D.

Dr. Claudio Maldonado, Ph.D.

Dr. Marsha Cole, Ph.D.

Dr. Corey Watson, Ph.D. 


\section{DEDICATION}

This dissertation is dedicated to my mother, Mrs. Kaberi Majumder, for supporting and encouraging me throughout my entire life. 


\section{ACKNOWLEDGEMENTS}

I would like to thank my mentor, Dr. Suresh C. Tyagi for his continuous support and encouragement throughout my time in his laboratory. His mentorship and passion for scientific understanding are truly unsurpassed. I would also like to thank him for believing in me during my doctoral program, and it was a privilege to work and learn under his able guidance and constant supervision. I am grateful to Dr. Clark for serving in my Ph.D. thesis committee and for her support during my doctoral studies. I would like to thank Dr. Maldonado for providing valuable insight and advice throughout my research endeavors. I would like to thank Dr. Cole for serving on my committee and for elevating my personal understanding of biochemistry to new heights. I would like to thank Dr. Watson for serving on my committee and for all of his support and advice over the years. I also would like to extend my most sincere gratitude to all of the professors in the Biochemistry \& Molecular Genetics Department and Departments of Physiology for their remarkable academic instruction. I would like to thank the administrative staff at the Department of Physiology as well as Biochemistry \& Molecular Genetics for all of their assistance over the years.

I would like to thank all of the members of my lab for providing a supportive network, a collegial environment and years of friendship. Special thanks to Mrs. Naira Metreveli, Dr. Mahavir Singh, and Dr. Neetu Tyagi for their continuous assistance and advice during my time in the lab. I would like to extend a very special thanks to Dr. Sen for his assistance, guidance, and friendship that have been invaluable for my success as a doctoral student. He has my deepest gratitude.

I would like to thank all of my friends and family for their unwavering support. 
Thanks to my sister, Mrs. Amrita Maji, for her support and encouragement over the years. Very special thanks to my mother, Mrs. Kaberi Majumder, and my father, Mr. Biswanath Majumder, for providing me the unwavering love and support in everything I do. I want to convey to them that they have been an inspiration to me throughout my life; thank you, my dear parents. Lastly, I would like to thank my incredible wife, Rani, for always supporting me during tough times and for making my life better in every way imaginable. 


\title{
ABSTRACT \\ EFFECTS OF HYDROGEN SULFIDE IN HYPERHOMOCYSTEINEMIA-MEDIATED SKELETAL MUSCLE MYOPATHY
}

\author{
Avisek Majumder
}

November $20^{\text {th }}, 2018$

\begin{abstract}
Although hyperhomocysteinemia (HHcy) occurs due to a deficiency in cystathionine- $\beta$-synthase (CBS), causing skeletal muscle myopathy, it is still unclear whether this effect is mediated through oxidative, endoplasmic reticulum (ER) stress, both or something else. Cystathionine $\mathrm{y}$-lyase (CSE) and CBS are the main $\mathrm{H}_{2} \mathrm{~S}$ producing enzymes, which produce Hydrogen sulfide $\left(\mathrm{H}_{2} \mathrm{~S}\right)$ from Hcy in the transsulfuration reaction. $\mathrm{H}_{2} \mathrm{~S}$ is an anti-oxidant compound and patients with a CBS deficiency cannot produce enough $\mathrm{H}_{2} \mathrm{~S}$, thus rendering them vulnerable to excess Hcymediated damage.

Both in-vitro (C2C12 cells) and in-vivo (CBS ${ }^{+/-}$mice) models were used to study HHcy-mediated muscle myopathy, and they were treated with sodium hydrogen sulfide (NaHS, an $\mathrm{H}_{2} \mathrm{~S}$ donor) to mitigate the effect(s) of HHcy in skeletal muscle. To examine whether muscle myopathy is due to poor angiogenesis, we created a hind limb femoral artery ligation (FAL) in $\mathrm{CBS}^{+-}$mouse model and treated them with GYY4137 (a longacting $\mathrm{H}_{2} \mathrm{~S}$ donor compound).
\end{abstract}


Results showed that oxidative stress was reversed by $\mathrm{NaHS}\left(\mathrm{H}_{2} \mathrm{~S}\right.$ donor) in Hcytreated C2C12 cells. ER stress markers (GRP78, ATF6, and pIRE1 $\alpha$ ) were elevated both in-vivo and in-vitro, and NaHS mitigated these effects. JNK-phosphorylation was upregulated in $\mathrm{C} 2 \mathrm{C} 12$ cells after Hcy treatment, but NaHS could not reduce this effect. Apoptosis was induced after Hcy treatment, and it was mitigated by the NaHS supplementation in $\mathrm{C} 2 \mathrm{C} 12$ cells.

HIF1- $\alpha$, VEGF, PPAR-y and p-eNOS expression levels were attenuated in skeletal muscle of $\mathrm{CBS}^{+/-}$mice after 21 days of FAL in comparison to WT mice and were improved via the GYY4137 (another $\mathrm{H}_{2} \mathrm{~S}$ donor compound) treatment. Collateral vessel density and blood flow were significantly reduced in post-FAL $\mathrm{CBS}^{+/-}$mice compared to WT mice, and these effects were ameliorated by the GYY4137 treatment. Plasma nitrite levels were decreased in post-FAL $\mathrm{CBS}^{+/-}$mice compared to WT mice, and these were improved by GYY4137 supplementation.

I also noticed FOXO1-mediated MuRF-1 upregulation, which further degrades MHC-I in $\mathrm{CBS}^{+/-}$mice compared to WT mice, and these effects were improved by $\mathrm{NaHS}$ intervention. Functional studies revealed that NaHS administration improved muscle fatigability in $\mathrm{CBS}^{+/-}$mice.

This work provides evidence that $\mathrm{H}_{2} \mathrm{~S}$ is beneficial in mitigating $\mathrm{HHcy}$-mediated skeletal muscle injury incited by oxidative or ER stress responses and $\mathrm{H}_{2} \mathrm{~S}$ might be employed as a potential therapeutic to alleviate the harmful metabolic effects of $\mathrm{HHcy}$ conditions. 
ACKNOWLEDGEMENTS..................................................................

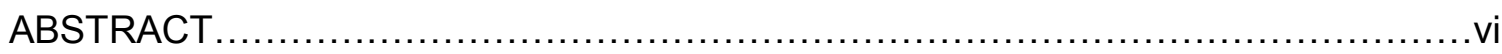

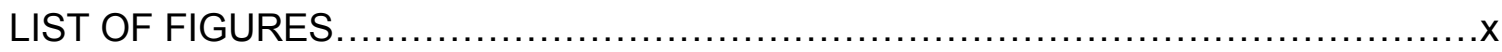

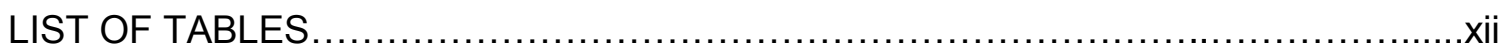

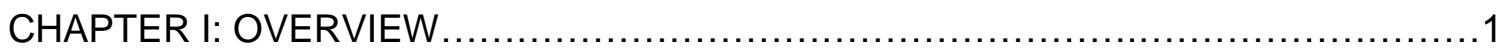

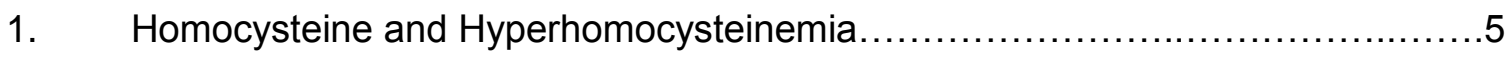

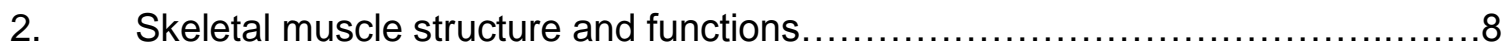

3. Hyperhomocysteinemia-mediated skeletal muscle pathophysiology.............10

3.1. Hyperhomocysteinemia-mediated oxidative/ER-stress responses..............12

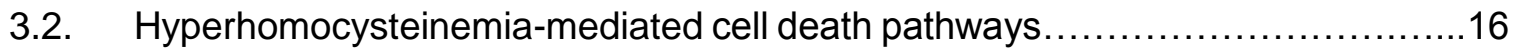

3.3. Hyperhomocysteinemia-mediated dysregulation of angiogenesis...............18

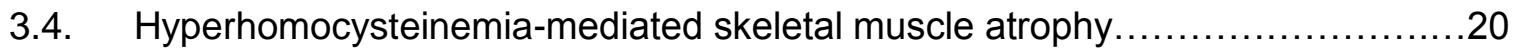

4. Effects of hydrogen sulfide on hyperhomocysteinemia-mediated

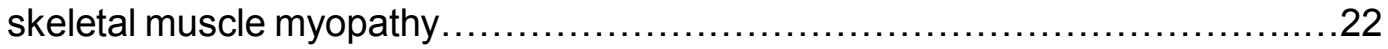

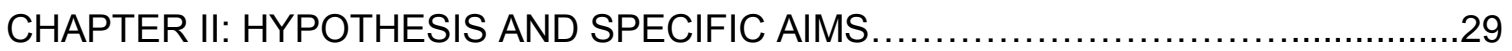

CHAPTER III: HYDROGEN SULFIDE ALLEVIATES HHCY-MEDIATED OXIDATIVE AND ER-STRESS RESPONSES IN SKELETAL MUSCLE

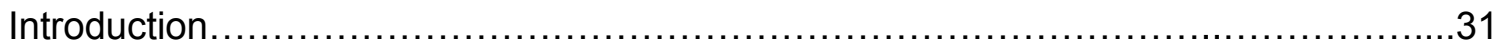

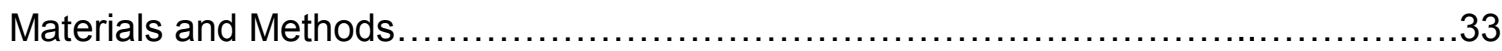




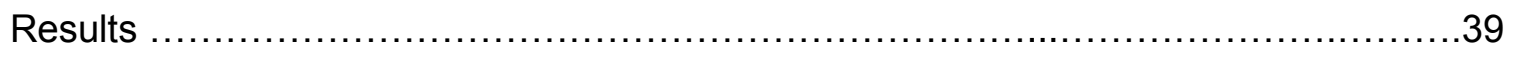

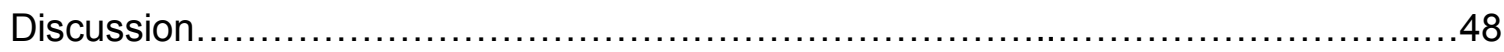

CHAPTER IV: HYDROGEN SULFIDE IMPROVES POST-ISCHEMIC ANGIOGENIC DEFECT IN THE HINDLIMB OF CYSTATHIONINE- $\beta$-SYNTHASE MUTANT MICE

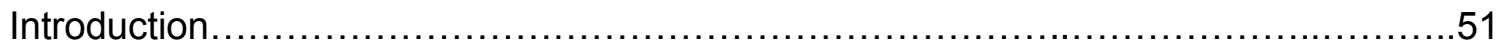

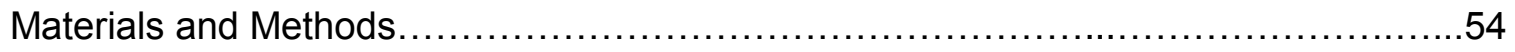

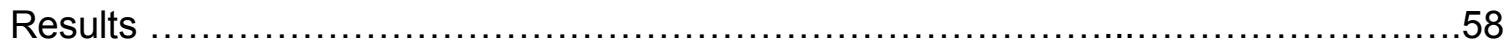

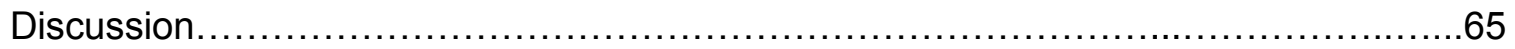

CHAPTER V: HYDROGEN SULFIDE MITIGATES HHCY-MEDIATED SKELETAL MUSCLE ATROPY VIA JNK/FOXO1 AXIS

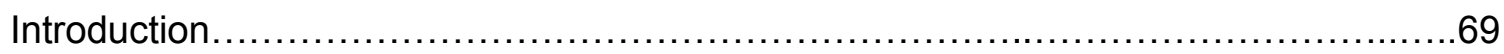

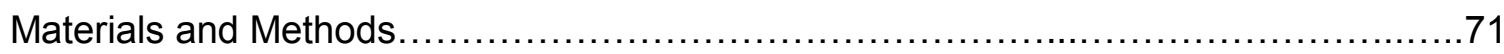

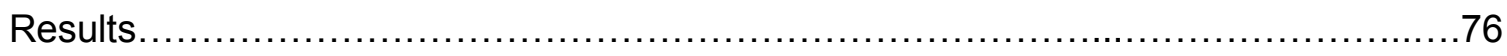

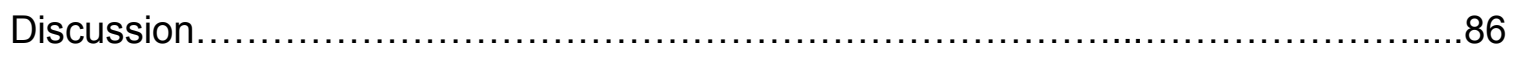

CHAPTER VI: SUMMARY AND FUTURE DIRECTIONS

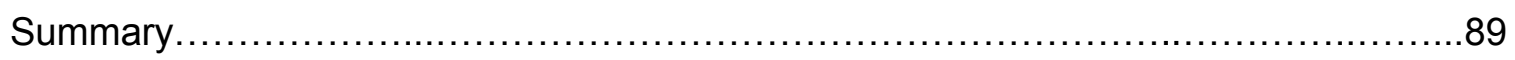

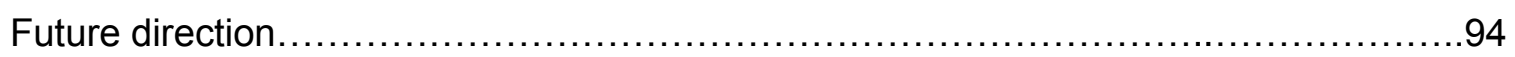

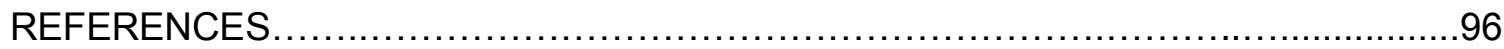

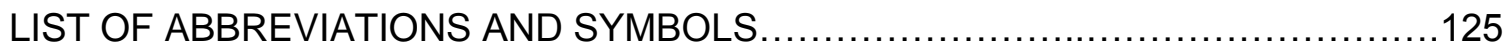

APPENDIX I: Physiological parameters of experimental mice ........................127

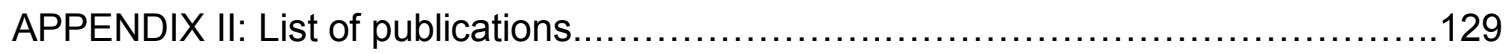

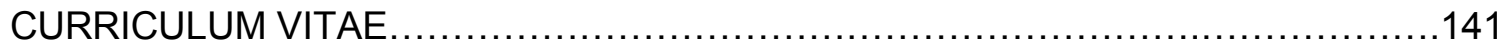




\section{LIST OF FIGURES}

Figure

PAGE

1. Schematic representation of homocysteine metabolism 1

2. A schematic highlighting hyperhomocysteinemia mediated skeletal

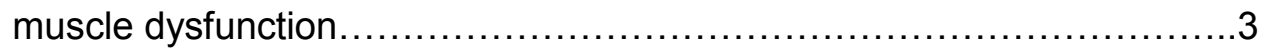

3. Structure of muscle fiber, myofibril and banding pattern..................9

4. Redox imbalance during hyperhomocysteinemia.......................13

5. HHcy-mediated cellular stress responses............................16

6. Hypoxia mediated angiogenesis via VEGF/Akt axis..................19

7. Schematic model of HHcy-induced muscle atrophy via nuclear

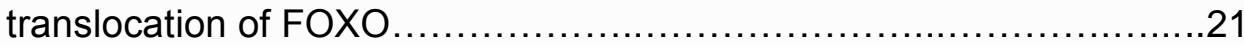

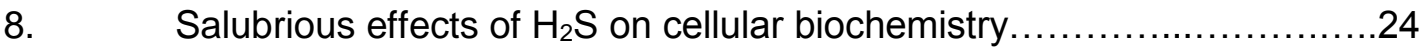

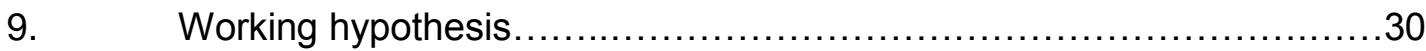

10. NaHS protects against Hcy-induced oxidative stress in $\mathrm{C} 2 \mathrm{C} 12$ cells......40

11A-D. High Hcy mediates ER-stress responses in C2C12 cells.................42

11E-H. High Hcy mediates ER-stress responses in muscle in-vivo..............43

12. High Hcy induces JNK-phosphorylation in both in-vitro and in-vivo........44

13. HHcy induced pro-inflammatory milieu in CBS+Met mice in-vivo...........46

14. Hcy treatment on induces C2C12 Cell Death............................47

15. Phenotypic and genotypic correlations between CBS $^{+/-}$and WT mice....59

16A-B. Effect of GYY4137 on the improvement of angiogenic markers in skeletal muscle of post-FAL mice 
16C-D. Effect of GYY4137 on angiogenic markers in skeletal muscle in sham

mice.

17A-D. GYY4137 supplementation improves neoangiogenic profile in the hindlimb muscle after 21 days of $\mathrm{FAL}$ in $\mathrm{CBS}^{+/}$mice

17E-G. Effect of GYY4137 supplementation on eNOS-phosphorylation and plasma nitrite levels in experimental mice.

18. Genotype-phenotype correlation and HHcy-mediated muscle mass loss. .77

19. NaHS treatment improves muscle fatigability in CBS+Met mice 79

20. HHcy reduces cross-sectional areas (CSAs) of skeletal muscle fibers in $\mathrm{CBS}+$ met mice. 80

21. HHcy-induced fibrosis and collagen deposition in skeletal muscle invivo. .81

22. Sub-cellular localization of FOXO1 in $\mathrm{C} 2 \mathrm{C} 12$ cells. .83

23. Hcy enhanced Atrogin-1 and MuRF-1 expressions in the muscle. .84

24. HHcy mediates elevated expression of MuRF1 and degradation of its targets protein MHC-1 .85

25.

Proposed model. 


\section{LIST OF TABLES}

\section{TABLES}

PAGE

1. List of most prevalent genetic polymorphisms/mutations reported in HHcy

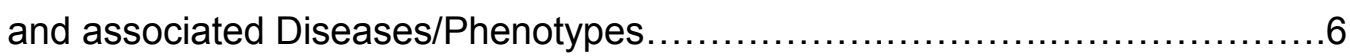

2. List of primers used for RT-qPCR experiments for chapter IV .................36

3. List of primers used for RT-qPCR experiments for chapter $\mathrm{V} \ldots \ldots \ldots \ldots \ldots \ldots \ldots 57$

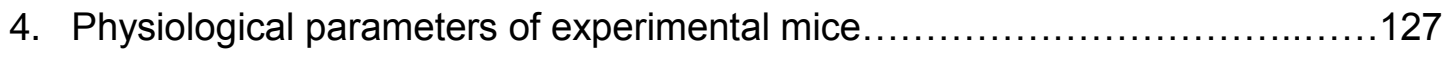

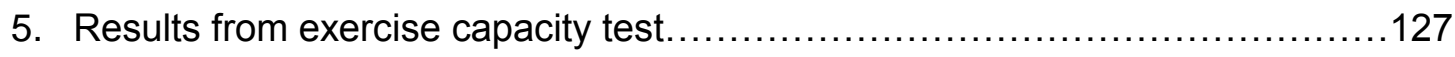




\section{CHAPTER I}

\section{OVERVIEW (1)}

Homocysteine (Hcy) has been studied extensively for over 30 years for its unique involvement in an increasing number of human diseases (2-4). It is generated via methionine cycle (MET-cycle) and its level is controlled by two processes: around $50 \%$ of Hcy goes to the transsulfuration pathway to produce cysteine, and the other half is remethylated back to methionine (MET) via the folate cycle (5-7) (Fig. 1). The normal total plasma Hcy concentration in the body is $5-15 \mu \mathrm{M}$, however, in a diseased condition, i.e., hyperhomocysteinemia (HHcy), total plasma Hcy levels increase (>15 $\mu \mathrm{M})(8)$. HHcy can be classified as moderate $(15-30 \mu \mathrm{M})$, intermediate $(30-100 \mu \mathrm{M})$ and severe $(>100 \mu \mathrm{M})$ $(7,8)$. There are mainly 4 ways humans can develop HHcy: (1) methionine-rich protein diet (9); (2) Vitamin $B_{12} /$ folate deficiency (10); (3) heterozygous/homozygous for cystathionine- $\beta$ synthase (11); and (4) obstruction of renal clearance (12).

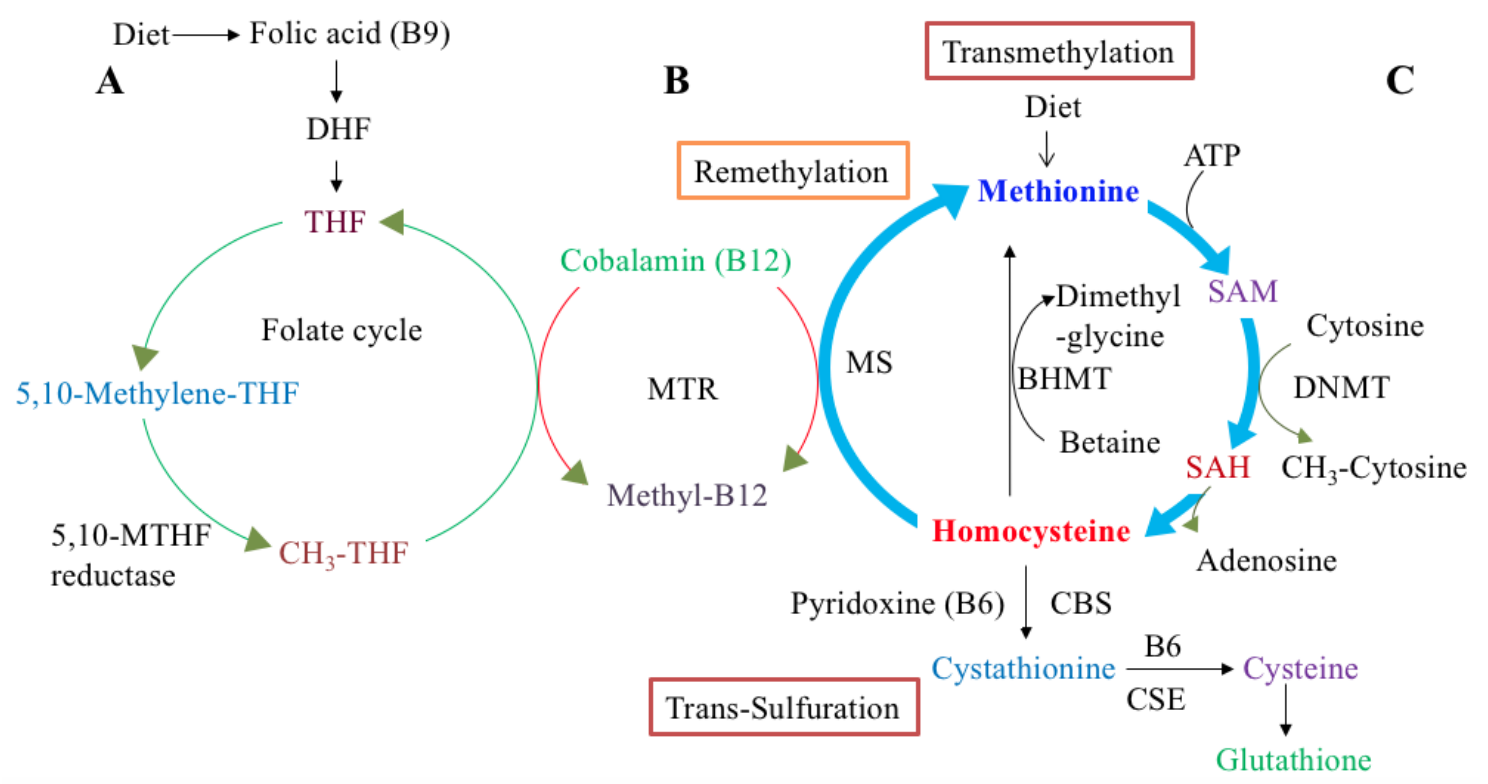


Figure 1: Schematic representation of Hcy metabolism. (A) Dietary methionine is converted to homocysteine (Hcy) through S-adenosyl methionine (SAM) and S-adenosyl homocysteine (SAH) and then back to methionine (MET). Hcy also bifurcates to the transsulfuration pathway where it is converted to cysteine in presence of rate-limiting enzymes cystathionine- $\beta$ synthase (CBS) and cystathionine- $\gamma$ lyase (CSE). Cysteine generated through transsulfuration pathway is further converted to glutathione $(\mathrm{GSH})$; (B) Conversion of cobalamin (Vitamin B12) to methyl-B12 in the presence of methionine synthase reductase (MTR) is necessary for remethylation of 5-methyl- tetrahydrofolate (THF) to THF; (C) Dietary folic acid (Vitamin B9) enters the folate cycle after its conversion first to dihydrofolate (DHF) and then to THF. The 5, 10methyltetrahydrofolate reductase (MTHFR) is a key enzyme that converts 5,10 methylene-THF to 5-methyl-THF.

HHcy has been associated with severe skeletal muscle dysfunction, but the precise mechanism(s) is still unknown $(7,13-20)$. Children born with very high levels of Hcy, i.e., HHcy caused by a lack of functional CBS alleles, show severe dysfunction of the musculoskeletal system and die shortly after birth, but individuals that heterozygous for non-functional CBS mutations can survive (13). Previous studies reported that $\mathrm{HHcy}$ could cause disruption of Z-discs, fiber size, and excessive collagen deposition in muscle $(15,20,21)$, but the pathways that trigger such pathological effects in skeletal muscles are not fully understood. Loss of skeletal muscle mass (muscle wasting) is mediated mainly by three processes: apoptosis, poor vasculature, and atrophy (protein degradation exceeds protein synthesis over time) $(22,23)$. Therefore, identification of the precise molecular mechanism(s) regulating the processes underlying $\mathrm{HHcy}$ is essential to devise proper treatment strategies. Findings from our group and other groups determined that HHcy induces oxidative stress in endothelial cells (24), vascular 
smooth muscle cells (25), liver tissue (26), and brain (27). Oxidative stress has been implicated in many diseases associated with protein misfolding and reduction of efficiency of protein folding pathways (28-33), but the cellular pathways that are involved in these stress-related conditions have not been elucidated. During severe ER-stress conditions, activated IRE1 recruits' TNF receptor-associated factor 2 (TRAF2) and apoptosis signal-regulating kinase 1 (ASK-1), which further activates c-Jun N-terminal kinase (JNK) (34-36) (Fig. 2). However, whether HHcy can compromise cell survival via activation of $\mathrm{JNK}$ is not studied previously. AKT is a major survival factor promoting cell proliferation, and its dysregulation has been detected in muscle wasting (37-39). A previous study showed that activated JNK could induce inhibitory phosphorylation of IRS-1 at Ser ${ }^{307}(40)$. Since Ser ${ }^{307}$ phosphorylation promotes general inhibition of IRS-1 signaling, it suggests that JNK-activation may impair PI3K/Akt axis (41). JNK controls cell response to the harmful extracellular stimulus $(42,43)$. JNK/c-Jun pathway regulates pro-apoptotic and pro-inflammatory gene expressions (44). Whether HHcy mediates oxidative and ER-stress responses, and whether this further induces apoptosis in muscle, has not been studied previously.

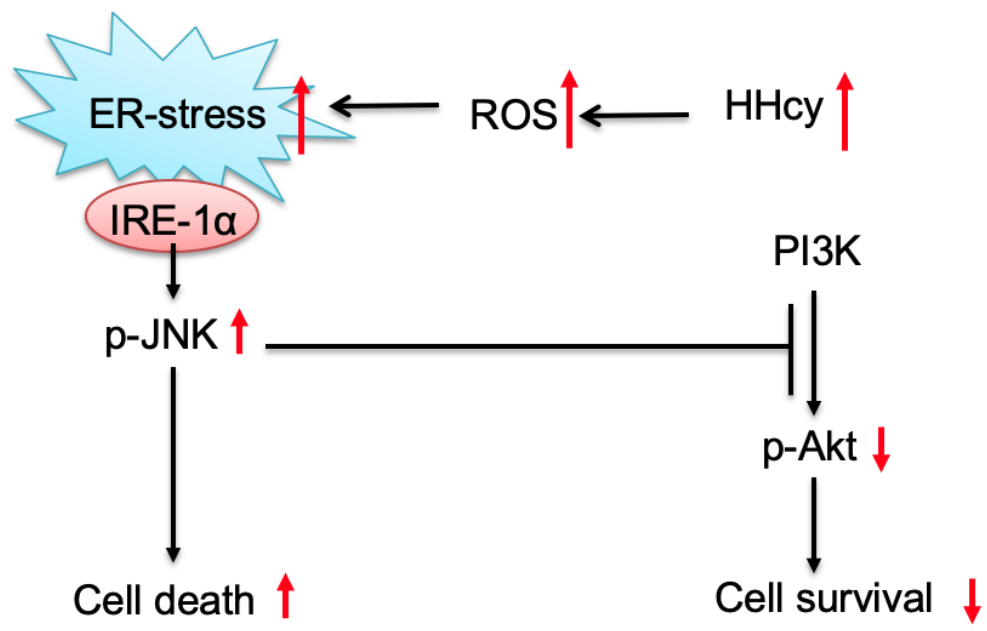

Figure 2: A schematic highlighting hyperhomocysteinemia mediated skeletal muscle dysfunction. HHcy can induce cell death via induction of ER and oxidative stress 
responses that can lead to skeletal muscle cell death via JNK-activation and concurrent impairment of PI3K/AKT survival axis.

$\mathrm{HHcy}$ is implicated in vascular toxicity, causing endothelial cell damage and dysfunction (20,45-47). Although several markers of ER-stress, including CCAATenhancer-binding protein homologous protein (CHOP), have been shown to be increased in a model of skeletal muscle atrophy, whether HHcy mediated ER-stress can play a role in muscle atrophy is still unclear (48). Additionally, several models of atrophy showed that inhibition of PI3K/Akt signaling induces nuclear import of FOXO in regulating Atrogin-1 and MurF-1 expression (49,50). Some studies have already demonstrated that HHcy inhibits Akt activation in endothelial cells (47), but whether this similar mechanism can be attributed to muscle myopathy is yet to be explored (37).

$\mathrm{H}_{2} \mathrm{~S}$ is increasingly being recognized as an important signaling molecule in the cardiovascular and nervous systems via its ability to neutralize a variety of reactive oxygen species (ROS) (51-53), as well as via increased cellular glutathione (GSH) levels through activation/expression of $\gamma$-glutamylcysteine synthetase (54-61). Cystathionine $\gamma^{-}$ lyase (CSE) and CBS are the main $\mathrm{H}_{2} \mathrm{~S}$ producing enzymes, which produce $\mathrm{H}_{2} \mathrm{~S}$ from Hcy in the transsulfuration reaction (62). CBS gene mutation leads to dysfunction in endogenous $\mathrm{H}_{2} \mathrm{~S}$ biosynthesis (63), suggesting that patients with this mutation are more prone to oxidative stress-mediated damages due to malfunction in Hcy metabolism and inefficient $\mathrm{H}_{2} \mathrm{~S}$ biosynthesis (54). CBS is a crucial enzyme in the transsulfuration pathway, and heterozygous CBS deficient $\left(\mathrm{CBS}^{+/-}\right)$mice show mild to severe endogenous elevation of circulatory Hcy levels (64-71) and lower levels of $\mathrm{H}_{2} \mathrm{~S}$ in their blood, which lead to oxidative stress-mediated damage $(72,73)$. Hence, it is important to study the mechanism of exogenous supplementation of $\mathrm{H}_{2} \mathrm{~S}$ as a possible approach to mitigate the effects of HHcy condition in skeletal muscle myopathy.

In this study, I examine the potential mechanisms of HHcy-mediated oxidative 
stress, ER stress, inflammation, apoptosis, angiogenesis, and atrophy in skeletal muscle, and whether $\mathrm{H}_{2} \mathrm{~S}$ has any beneficial role on mitigating these effects.

\section{Homocysteine and Hyperhomocysteinemia}

Homocysteine (Hcy) is a sulfur-containing non-proteinogenic amino acid that is produced during the metabolism of methionine (MET) via MET-cycle (2). Post absorption, MET is converted into S-adenosylmethionine (SAM) by ATP and catalyzed by methionine adenosyltransferase (MAT), leaving it with a transferrable methyl group. This methyl group is donated for cellular methylation requirements and becomes Sadenosyl-homocysteine (SAH). SAH hydrolase is an enzyme that reversibly hydrolyzes SAH and produces Hcy (74). Hcy is present in blood in three different forms: around 1\% as free thiol, $70-80 \%$ as a disulfide-bound to a swath of plasma proteins, and the remaining $20-30 \%$ as a homo/heterodimerize with other thiols (3). Upon the generation of Hcy via the MET-cycle, $50 \%$ goes to the transsulfuration pathway for production of glutathione (GSH, an antioxidant) and the rest is re-methylated back to MET via the folate cycle as shown in Fig. 1 (2,5,6).

In healthy individuals, the generation and elimination of Hcy stays in balance; however, once this homeostasis is disturbed, it leads to HHcy (75). HHcy can be developed via consumption of an excess amount of MET-rich diet, vitamin $B_{12} /$ folate deficiency, the occurrence of heterozygous/homozygous CBS gene mutation, and the obstruction of renal clearance (12). Besides these, genetic mutations in any Hcy metabolism pathway-regulated genes (e.g., 677C >T and 1298A>C in the MTHFR gene) can also lead to HHcy (14,76-78) (Table 1). Other determinants as well, such as age, sex, physical activity, alcohol intake, certain medications, and even different disease conditions can offset the rate of MET-cycle, thereby influencing the corresponding increase in the total Hcy levels in the blood (79). During HHcy, the MET-cycle is 
generally dysregulated (80). This leads to disruption of multiple signaling pathways because it is the only pathway that gives rise to the production of essential methyl groups needed for the subsequent biosynthesis of cellular compounds such as creatine, epinephrine, carnitine, phospholipids, proteins, and polyamines and also the epigenetically governed cellular processes like methylation of DNA, RNA, and histones that are dependent upon Hcy metabolism (7,81). Although HHcy conditions can cause multi-organ pathologies via oxidative and ER-stress responses $(7,20,82-84)$, the precise mechanisms of multi-organ pathology due to $\mathrm{HHcy}$ remain elusive.

\begin{tabular}{|c|c|c|c|c|c|}
\hline \multirow[b]{2}{*}{ Genes } & \multicolumn{2}{|c|}{ Polymorphisms/Mutations } & \multirow[b]{2}{*}{ Condition } & \multirow[b]{2}{*}{$\begin{array}{c}\text { Associated } \\
\text { Diseases/Phenotypes }\end{array}$} & \multirow[b]{2}{*}{ References } \\
\hline & $\begin{array}{l}\text { Nucleotide } \\
\text { change }\end{array}$ & $\begin{array}{l}\text { Amino acid } \\
\text { /splice site } \\
\text { change }\end{array}$ & & & \\
\hline \multirow{3}{*}{ CBS } & T833C & Ile278Thr & HHcy & Stroke & (86) \\
\hline & $844 I N S 68$ & $\begin{array}{c}\text { produces an } \\
\text { alternate splice } \\
\text { site }\end{array}$ & HHcy & $\begin{array}{c}\text { Peripheral artery occlusive } \\
\text { disease }\end{array}$ & (85) \\
\hline & $844 I N S 68$ & $\begin{array}{c}\text { produces an } \\
\text { alternate splice } \\
\text { site }\end{array}$ & HHcy & Thrombosis & (87) \\
\hline \multirow{10}{*}{ MTHFR } & C677T & Ala223Val & HHcy & Retinal vein occlusion & (88) \\
\hline & C677T & Ala223Val & HHcy & Stroke & $(89-92)$ \\
\hline & C677T & Ala223Val & HHcy & Venous thromboembolism & (93) \\
\hline & C677T & Ala223Val & HHcy & Hypertension & $(94-96)$ \\
\hline & C677T & Ala223Val & HHcy & Alzheimer's Disease & (97) \\
\hline & A1298C & Glu429Ala & HHcy & $\begin{array}{c}\text { Cerebral venous sinus } \\
\text { thrombosis }\end{array}$ & $(98-100)$ \\
\hline & C677T & Ala223Val & HHcy & Hyperlipidemia & (101) \\
\hline & C677T & Ala223Val & HHcy & Diabetic nephropathy & $(102-105)$ \\
\hline & C677T & Ala223Val & HHcy & $\begin{array}{l}\text { Cerebral venous } \\
\text { thrombosis }\end{array}$ & $(106)$ \\
\hline & C677T & Ala223Val & HHcy & Parkinson's Disease & $(107,108)$ \\
\hline
\end{tabular}


Table 1. List of most prevalent genetic polymorphisms/mutations reported in HHcy and associated Diseases/Phenotypes.

Metabolism of Hcy depends on several factors, i.e., the level of methionine in the diet, SAM level and the type of cells in which methionine metabolism takes place (109). High SAM level can act as an allosteric inhibitor for methylenetetrahydrofolate reductase (MTHFR). MTHFR catalyzes the conversion of 5,10-MTHF to 5-MTHF, which is a cosubstrate of Hcy in the remethylation reaction (110). Therefore, the presence of high SAM prevents Hcy from entering the remethylation pathway. High SAM level also acts as an allosteric activator CBS the transsulfuration pathway $(111,112)$. Together it appears that the presence of high level of SAM favor Hcy entering to the transsulfuration pathway. However, only a few organs such as the liver, kidney, pancreas, brain, adipose tissue and small intestine express CBS $(113,114)$. Vascular and muscle tissues don't express CBS, so the high SAM lead to transient accumulation of Hcy (82). Hcy metabolism also depends on dietary MET load (115-117). When diet contains a basal methionine level, Hcy cycles through remethylation pathway about 1.5-2.0 times before being directed towards the transsulfuration pathway, but when dietary methionine content is half the basal level, the cycling of Hcy via the remethylation pathway increases 2-fold (118). Conversely, when the dietary MET level is high, Hcy cycling through the remethylation is reduced by about 1.5 -fold (118). High levels of intracellular Hcy exported out into the circulation (119); however, the exact mechanism of Hcy export is not identified yet. Although HHcy is found in $~ 5-7 \%$ of the general population and is associated with increased risk of cardiovascular disease, osteoporosis, skeletal muscle myopathy and other metabolic complications $(7,20,82-84)$, the precise molecular pathways that lead to multi-organ dysfunction remain mostly unexplored. 


\section{Skeletal muscle structure and functions}

Skeletal muscle is the largest organ (roughly 40 to $50 \%$ of the body's total weight in an average human), constitutes $50-75 \%$ of the body's total proteins (120-122). Muscle contains very long multinucleated cells (myocytes) that are bundled up and surrounded by connective tissues (123). Primary functions of skeletal muscle are: 1) production of contractile force that provides breathing, locomotion and postural support; and 2) thermogenesis during periods of cold stress (123). For force production, depending on the primary function of the muscle it consists of either fast twitch (type I) or slow twitch (type II) fibers (123). Type I fibers are used for repetitive, low-intensity functions, such as postural support (123). These fibers provide less force generation but are fatigueresistant and have a higher reliance on oxidative metabolism and therefore have more density of mitochondria and oxidative enzymes (123). Type II fibers are divided into two fiber subsets: type IIx and type Ila (123). Type IIx fibers are very glycolytic and produce high contractile force but are susceptible to fatigue due to a lower mitochondrial density (123). Type lla fibers are an intermediate fiber type (123). They provide a moderate contractile force and are more fatigue-resistant than Type Ilx fibers (123).

Myocytes, unlike most other cell types, contain multiple nuclei and are striated because of contractile segments called sarcomeres (123). Within each muscle fiber, a structure called myofibril that contains the contractile protein of the muscle called actin and myosin (123). Actin filament consists of two additional proteins known as troponin and tropomyosin, which are used for contractile regulation (123). The striated regions, called sarcomeres, are within the myofibrils and consist of areas of myosin/actin overlap, as well as an area where myosin does not overlap with actin, called the $\mathrm{H}$ zone (123) (Fig. 3). Sarcomeres are divided by a wall of structural proteins, which makes up a region of the sarcomere called the $Z$ line (123). Based on "sliding filament theory," during 
contraction, the required energy comes from the de-phosphorylation of adenosine triphosphate (ATP), wherein myosin heads "cock" form cross-bridges with actin and then pivot inward towards the $\mathrm{H}$ zone (124), that ultimately decreases the sarcomere length from one $Z$ line to another $Z$ line (Fig. 3) (125).
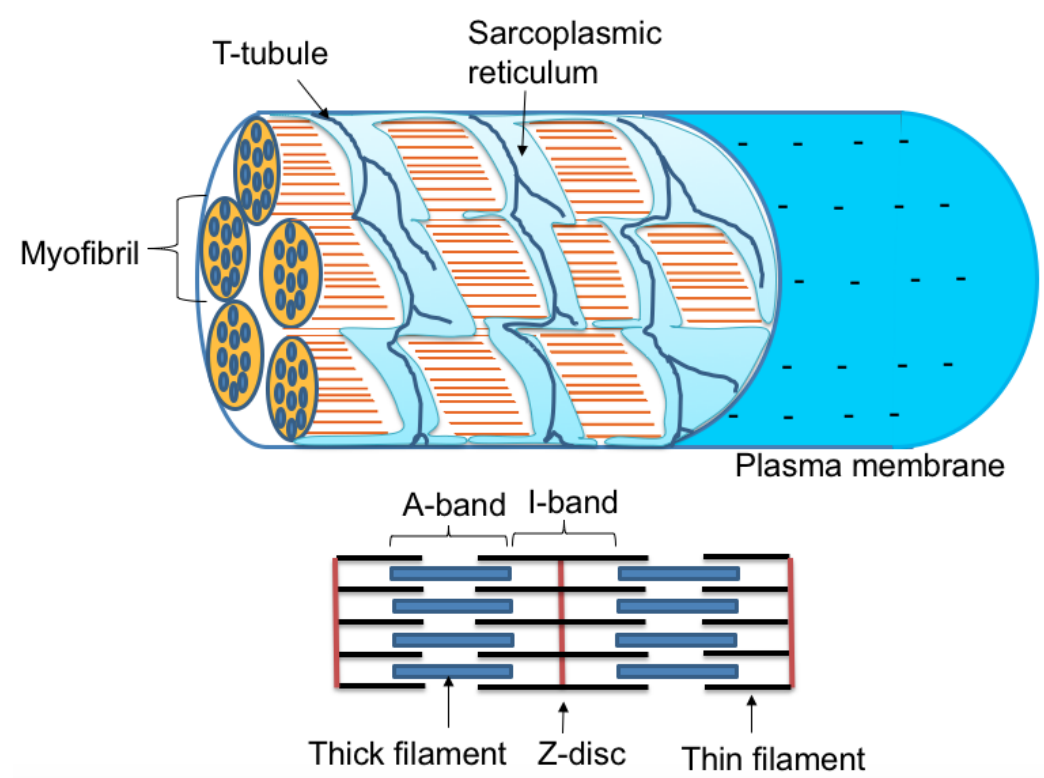

Figure 3: Structure of a muscle fiber, organization of myofibril, and banding pattern.

Surrounding each myofibril is a network of channels that serve as a storage site for calcium, which is crucial for the regulation of muscle contraction (126). This structure is called the sarcoplasmic reticulum (126). The transverse tubules are another set of channels that pass through the muscle fiber (126). Each myocyte is connected to a branch of a motor neuron through a neuromuscular junction serving as the site of electrochemical transmission (127). Collectively, all the myocytes that are innervated by a single motor neuron are termed as a motor unit (127). Upon generation of an action potential high enough to cause contraction, the neural impulse is transmitted to the myocyte through the neuromuscular junction and is propagated down the transverse tubules to the sarcoplasmic reticulum, causing $\mathrm{Ca}^{++}$to be released (128). $\mathrm{Ca}^{++}$binds to the troponin molecule, which creates a shift in tropomyosin position (128). This allows for 
exposure of the actin active sites and myosin-actin cross bridge formation, resulting in contraction (128).

In adults, repair of degenerated muscles relies on a small population of skeletal muscle stem cells known as satellite cells (129). Satellite cells, a population of quiescent muscle precursor cells, reside beneath the basal lamina, providing the predominant source of additional myonuclei for muscle growth $(130,131)$. Once activated, satellite cells give rise to myoblasts that proliferate, differentiate, and fuse to form new muscle fibers or to repair damaged muscle fibers $(132,133)$. The availability of a pool of myoblasts for myogenesis is essential for the development of muscle (134). The decreased number of muscle fibers could be due to the reduced myoblast proliferation or cytotoxicity (134). Moreover, in most cases, the apoptosis of myoblasts serves as a physiological behavior to remove excess myoblasts during myogenesis or muscle regeneration, while inappropriate apoptosis will pathologically lead to degeneration that is associated with various muscular dystrophies and atrophies $(135,136)$. Therefore, identification of the molecular mechanism(s) of how high Hcy induced myoblast cytotoxicity is essential in understanding skeletal muscle growth, disease, and regeneration during HHcy condition.

\section{Hyperhomocysteinemia-mediated skeletal muscle pathophysiology}

$\mathrm{HHcy}$ is a known risk factor for vascular diseases (137); however, several studies also reported that $\mathrm{HHcy}$ leads to skeletal muscle weakness and functional impairment $(5,15,20,21,46,66,82)$. Children born with severe homocystinuria due to CBS deficiency exhibit reduced body weight, skeletal muscle myopathy, and die teenage years (7) $(5,15,20,21,46,66,82)$. Previous studies from our group showed that genetically modified HHcy mice (heterozygous for CBS gene deficiency) have lower body weights and less fatigue resistance and produce less contractile force, contain lower muscle ATP 
levels, low dystrophin, and mitochondrial transcription factor A (mtTFA) (82). Similarly, in a study, Kanwar et al. [1976] found that HHcy can cause focal fragmentation, disruption, and smearing of the Z-discs and disorganization of the myofilaments in the skeletal muscles (15). This study also showed that HHcy could reduce cellular metabolic activity and induce energy imbalance in gastrocnemius rat skeletal muscle by decreasing the activity of pyruvate kinase, creatine kinase, and noticed increased activity of succinate dehydrogenase (18). Similarly, Veeranki et al. [2015] showed that Hcy affects fatty acid oxidation, affecting energy metabolism in skeletal muscle via PGC-1a specific protein nitrotyrosylation and a concomitant reduction in association with PPAR-y (82). On the other hand, many neurological disorders like amyotrophic lateral sclerosis (ALS) and multiple sclerosis affect muscle degeneration, and these medical conditions are also connected to HHcy (16). HHcy significantly lowers physical functions by deteriorating skeletal muscle functions in older compared to age-matched healthy subjects (138), which suggests that aging is one of the potential risk factors to be considered in this process (139).

Disorders, such as muscular dystrophy, sarcopenia, and immobilization, have been extensively researched. However, there is not enough data available considering the effects of HHcy on these conditions. Swart et al. [2013] demonstrated that in elderly individuals ( 75.6 years of age), plasma Hcy levels had a strong inverse correlation with grip strength and functional capabilities (140). High Hcy levels resulted in poor grip strength in men and lowered functional ability including walking, climbing stairs and rising from a chair (140). In heterozygous CBS-deficient mice $\left(\mathrm{CBS}^{+/}{ }^{+-}\right.$, a model of mild to moderate $\mathrm{HHcy}$, it was observed that 28 days post-induction of hindlimb ischemia $\mathrm{CBS}^{+/-}$ mice displayed blunted hindlimb perfusion and lower collateral blood vessel development when compared to WT mice (46). This study also found that $\mathrm{CBS}^{+/-}$mice had lower vascular endothelial growth factor (VEGF), hypoxia-inducible factor (HIF1- $\alpha$ ) 
and peroxisome proliferator-activated receptor coactivator 1- $\alpha$ (PGC1- $\alpha)$ after 28 days of ischemic recovery when compared to controls (46). Below, I have summarized the findings relevant to oxidative/ER-stress, apoptosis, atrophy and poor angiogenesis caused by HHcy that might contribute to skeletal muscle dysfunction.

\subsection{HHcy-mediated oxidative/ER-stress responses in skeletal muscle}

During metabolic processes, a certain amount of pro-oxidative reactive oxygen species (ROS) are formed. The intracellular environment is predominantly a reducing environment due to the presence of enzymes like glutathione peroxidase (GPx), superoxide dismutase (SOD), and catalase (CAT), which break down ROS into water and oxygen. There are also a series of built-in redox defense systems that inactivate ROS, which include reduced glutathione $(G S H)$, hydrogen sulfide $\left(\mathrm{H}_{2} \mathrm{~S}\right)$, nicotinamide adenine dinucleotide - hydrogen (reduced) (NADH), thioredoxin, and free radical scavengers such as Vitamins $C$ and $E(141-143)$. Oxidative stress occurs when there is an imbalance between free radical production and antioxidant capacity in a given cell. Previous studies revealed that Hcy contains a -SH group like other thiols (RSH) which can undergo oxidation to form a disulfide bond (RSSR) even at physiological pH in the presence of metal catalysts and molecular oxygen $\left[\mathrm{O}_{2}{ }^{\circ}\right]$ (21) (Fig. 4). Further, Hcy can also produce hydrogen peroxide $\left(\mathrm{H}_{2} \mathrm{O}_{2}\right.$, a pro-oxidant molecule) during metal-catalyzed oxidation step and peroxynitrite (ONOO', a potent oxidant) in the presence of nitric oxide (NO) and superoxide anion $\left(\mathrm{O}_{2}^{-}\right)$(144). Findings from our group and others demonstrated HHcy effects on oxidative stress in cardiac microvascular endothelial cells (24), vascular smooth muscle cells (25), and the liver tissue (26). Although these phenomena have been studied in multiple tissue types, whether HHcy exerts its detrimental effects on muscle through similar mechanisms is not known. 


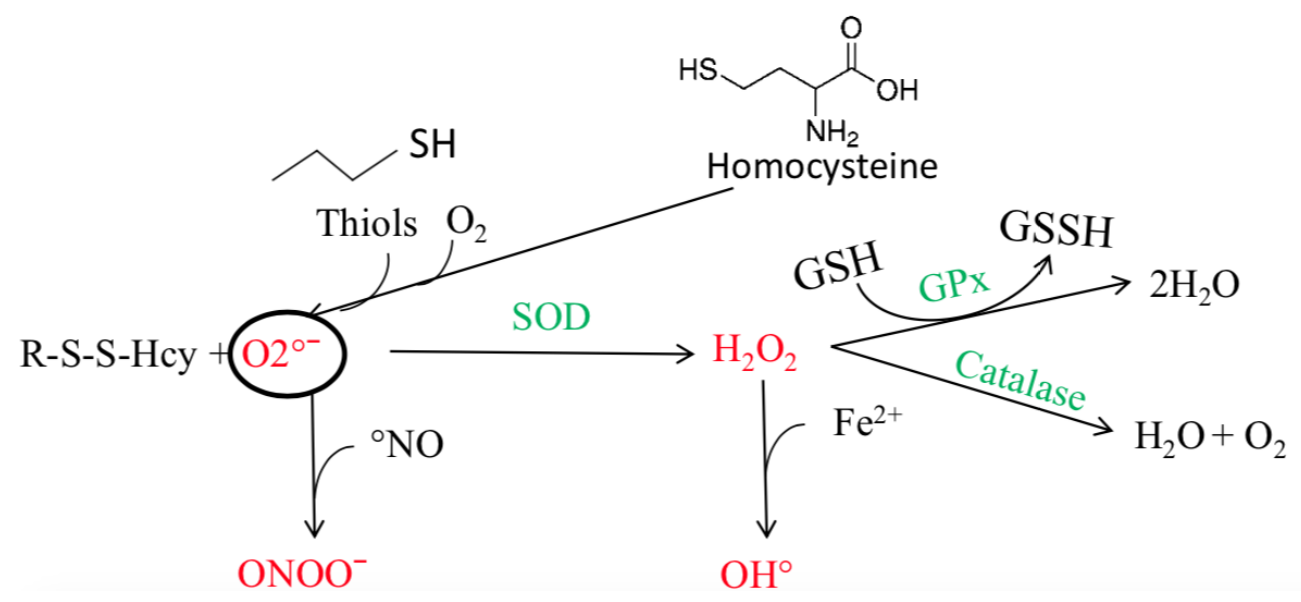

Figure 4: Redox imbalance during hyperhomocysteinemia: (A) Homocysteine can homo/hetero-dimerize in the presence of transition metal catalysts and molecular oxygen to form superoxide radicals that are converted to $\mathrm{H}_{2} \mathrm{O}_{2}$ by superoxide dismutase (SOD) and which are in turn neutralized to $\mathrm{H}_{2} \mathrm{O}$ and $\mathrm{O}_{2}$ by glutathione peroxidase (GPx) and catalase (CAT).

Several studies have reported that HHcy creates oxidative stress that promotes apoptosis, inflammation, insulin resistance, and dysregulation of lipid metabolism in different organs $(5,7,145,146)$. Moreover, increased ROS level due to HHcy can be involved in lipid peroxidation, protein denaturation, and DNA damage, which ultimately damage cellular components; modulates gene expression, alters cell signaling pathways, and energy imbalance (147). Besides, Hcy can also form Hcy-thiolactone and acetylates free amino groups in proteins, which further intensify the exaggerated oxidative stress condition (146). DiBello et al. [2010] showed that proteins (PRDX1, PRDX2, and HSP90AA) which are produced as a response to oxidative stress were significantly upregulated in the liver during HHcy (148). One study showed that production of ROS was ameliorated by PPAR-y activation in ECs (146), as reported previously from our laboratory indicating that PPARY was reduced in HHcy condition 
(148), suggesting that PPAR-y can be used as a potential target to mitigate oxidative stress during HHcy. HHcy lead to oxidative stress and that interferes with different cellular signaling pathways in various organs; therefore, it suggests that HHcy may have similar pathological effect in muscle tissue.

Oxidative stress has been implicated in many diseases associated with protein misfolding pathways (28-33). Indeed, a study by Malhotra et al. [2008] showed that antioxidants reduce endoplasmic reticulum (ER) stress and improve protein secretion in Chinese hamster ovary (CHO)-H9 cells (149). Similarly, a study using differential display analysis in HUVECs found that GRP78 (78-kDa glucose-regulated protein) was upregulated in Hcy-treated HUVECs compared to controls (150). GRP78 is a chaperone protein belonging to the HSP70 family and predominantly resides in the lumen of the ER (151). HHcy can induce ER-stress (33), but which pathways get affected by this condition is poorly understood. It is known that after translation protein folding occurs inside ER, but during stress conditions, misfolded proteins accumulate inside the ERlumen and induce unfolded protein response (UPR) (152). UPR increases transcription of chaperone genes to facilitate protein folding, helps to attenuate the translation process, and finally, it degrades misfolded proteins accumulated in ER (153-155). In the mammalian system, UPR is mediated through inositol-requiring protein 1 (IRE1), PRKRlike ER kinase (PERK), and activating transcription factor 6 (ATF6) (156). IRE1 promotes X-box binding protein 1 (XBP1) mRNA splicing, which finally activates ER chaperones (e. g. BiP/GRP78, EDEM, and ERdj4). PERK phosphorylates eukaryotic translation initiation factor $2 \alpha$ (elF2 $\alpha)$, and that stops translation. ATF6 regulates the expression of chaperone genes and ER-associated degradation (ERAD) genes (157159). Several studies showed that HHcy could exacerbate ER-stress by reducing the efficiency of protein folding pathways and increasing the production of misfolded proteins (28). ER-stress might play a key role in mediating adverse effects during the HHcy 
condition in muscle (160).

In the mammalian system, there are two isoforms of IRE1: IRE1 $\alpha$ and IRE1 $\beta$. Both of these proteins are transmembrane proteins and contain a Ser/Thr kinase domain and an endoribonuclease domain $(161,162)$. In a stress-free environment, IRE1 $\alpha$ remains in an inactivated form bound with GRP78; however, in stress condition, it dissociates from GRP78 and gets auto-phosphorylated (163). Then the endonuclease activity of activated IRE1 $\alpha$ cleaves a $26 b p$ intron from ubiquitously expressed XBP1u mRNA. Removal of this intron causes a frameshift in the XBP1 coding sequence, resulting in the translation of XBP-1s isoform (152). XBP-1s encodes a specific basic leucine zipper-containing transcription factor, known as X-Box Binding Protein-1 (XBP1) to promote cell survival (163-165). However, during severe ER-stress conditions, IRE1 $\alpha$ recruits' TNF receptor-associated factor 2 (TRAF2) and apoptosis signal-regulating kinase 1 (ASK-1), then activates C-Jun N-terminal kinase (JNK) and induces apoptosis (34-36). Although many studies also reported bidirectional regulation of ER-stress in apoptosis through JNK activation, and concomitant attenuation of cell proliferation and protein synthesis via PI3K/AKT axis (166-168), it is unknown whether HHcy exerts its detrimental effect in muscle through this similar mechanism pathway (Fig. 5). 
(A)

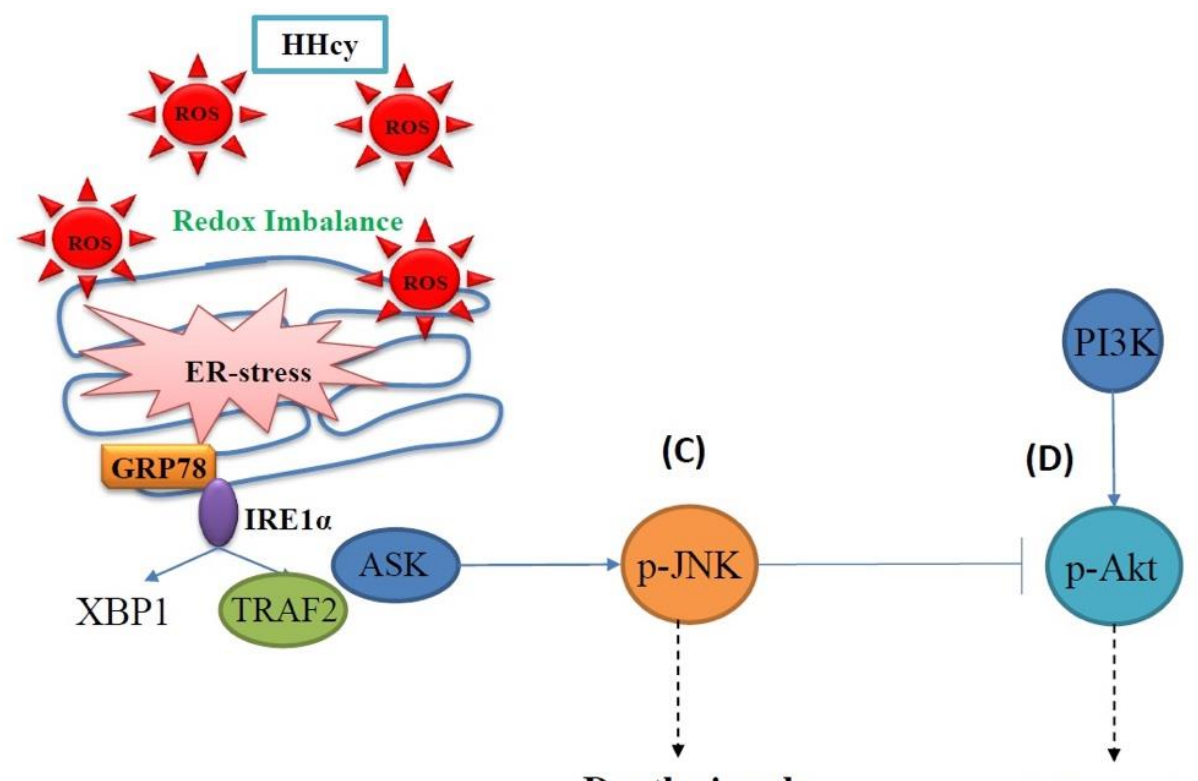

Death signals

Cell Survival

Figure 5: HHcy-mediated cellular stress responses. (A) HHcy-mediated excessive production ROS impaired biogenesis, as well as protein folding process leading to ERstress, (B) Activation of IRE1a during ER-stress response, causes XBP1 mRNA splicing. During severe ER-stress condition activated IRE1 $\alpha$ recruits' TNF receptor-associated factor-2 (TRAF2) along with apoptosis signal-regulating kinase-1 (ASK1), which further activate c-Jun N-terminal kinase (JNK). (C) Activated JNK phosphorylates c-Jun, that recruit activator protein-1 (AP-1) to the promoter sequences of various pro-apoptotic and pro-inflammatory genes and induce cell deaths. (D) JNK is also involved in the phosphorylation of IRS-1 at Ser ${ }^{307}$ which in turn inhibits IRS-1 signaling thus impairing the PI3K/AKT axis and interfere with cell survival.

\subsection{HHcy-mediated cell death pathways}

Apoptosis is programmed cell death and is required for normal development; however, a very high amount of apoptotic cell death due to environmental stress can be detrimental (169). Excessive apoptosis is observed in many well-known disease states such as ischemic heart disease, AIDS, Alzheimer's, and Parkinson's, etc. (170-172). 
Apoptosis occurs in 2 phases; namely, the death decision phase and execution phase. Death decision phase is controlled by two proteins, such as pro-apoptotic (Bax and Bak) and anti-apoptotic proteins (BCL-XL and BCL-2). The execution phase is regulated by caspases (proteolytic enzymes), responsible for the execution of apoptosis after the death decision is confirmed (173). Unlike necrosis, apoptosis occurs at a single cell level (173). Studies showed that an increase in oxidative stress could lead to caspase activation (174-177). This oxidative stress-mediated apoptosis was further supported by other studies demonstrating that anti-oxidants such as $\mathrm{N}$-acetylcysteine (NAC) treatment could block apoptosis in a similar way that caspase inhibitors do $(178,179)$. Several mechanisms(s) have been proposed pointing to the fact that oxidative stress generated by HHcy can lead to multiorgan pathologies $(5,7,145,146)$. This argues that stimulus no other than redox imbalance during HHcy is capable of inducing apoptotic cell death. Even though findings have suggested that HHcy could be causal in a wide range of disorders including skeletal muscle myopathy; however, whether Hcy mediates skeletal muscle myopathy via inducing different types of cell deaths (apoptotic, necrotic and pyroptotic) are needed to identify.

Studies showed that Hcy induced apoptotic cell death in ECs $(180,181)$. A previous finding revealed that Hcy causes apoptosis in HUVECs via activation of UPR, which was mediated through IRE1 activation (182). Similarly, another study suggested that Hcy can induce trophoblast cell death during embryonic development (183). Hcy was also found to induce apoptosis in bone marrow mesenchymal stem cells (BMSCs) via oxidative stress mediated JNK activation (184). Folate deficiency, which is one of the causes of HHcy found to induce apoptotic cell death in RINm5F pancreatic Islet $\beta$-cells, through oxidative-nitrosative stress (185)(186). Mesangial cell apoptosis via inducing ROS and p38-MAPK activation had also been reported (187). Hcy induces apoptosis via upregulation of DNA damage response in neurons; this process is mediated through 
PARP activation, and p53 induction (188). Our lab using genetically engineered heterozygous $\mathrm{CBS}$ deficiency mouse model $\left(\mathrm{CBS}^{+-}\right)$reported that $\mathrm{HHcy}$ causes cell death via upregulation of mitochondria-mediated cell death pathway (BAX, caspase-9, and caspase-3) (189). Hydrogen sulfide $\left(\mathrm{H}_{2} \mathrm{~S}\right)$ is known to be involved in the modulation of many physiological pathways. A study found that $\mathrm{H}_{2} \mathrm{~S}$-releasing sildenafil (ACS6) treatment could protect HHcy-mediated apoptosis in PC12 cells (190).

\subsection{HHcy-mediated dysregulation of angiogenesis}

Although many studies have been conducted to identify the possible mechanism(s) of skeletal muscle myopathy, fewer studies have demonstrated the potential risk factors that inhibit skeletal muscle adaptability in response to chronic ischemia. Poor angiogenic capacity is known to play a vital role in skeletal muscle adaptability $(191,192)$. Lack of revascularization is already reported in elderly frailty and in many metabolic disorders, where metabolic factors and associated signaling pathways are found to be involved in a variety of skeletal muscle dysfunctions (193). Besides these, $\mathrm{HHcy}$ is well-studied in the cardiovascular system where it has been shown to increase oxidative stress in vascular tissue and cause dysfunction of ECs $(194,195)$.

The purpose of capillaries is to serve as the interface for delivery of oxygen and removal of metabolites to/from tissues. Angiogenesis is a physiological process where new blood vessels form from the pre-existing ones (191). Angiogenesis plays a vital role during tissue injury/wound repair, endometrial cycle, and muscle adaptation to stress/exercise (196). Previous studies demonstrated that HHcy impairs angiogenesis in multiple tissues (197-201). During angiogenesis, the main angiogenic signal is vascular endothelial growth factor (VEGF). VEGF production is mainly up-regulated in a tissue under hypoxic conditions via inducing hypoxia inducing factor $1 \alpha(\mathrm{HIF} 1 \alpha)$ and that plays 
a pivotal role in angiogenesis. VEGF binds to VEGF receptor (VEGFR) on ECs' surface which goes through promotes ECs' growth and migration towards the source of VEGF (202). Upon VEGF binding to VEGFR, PI3K is also activated leading to activation of the protein kinase Akt, which in turn phosphorylates eNOS and increases NO production during angiogenesis as shown in Fig. 6. Akt activation can also promote endothelial proliferation and cell survival.

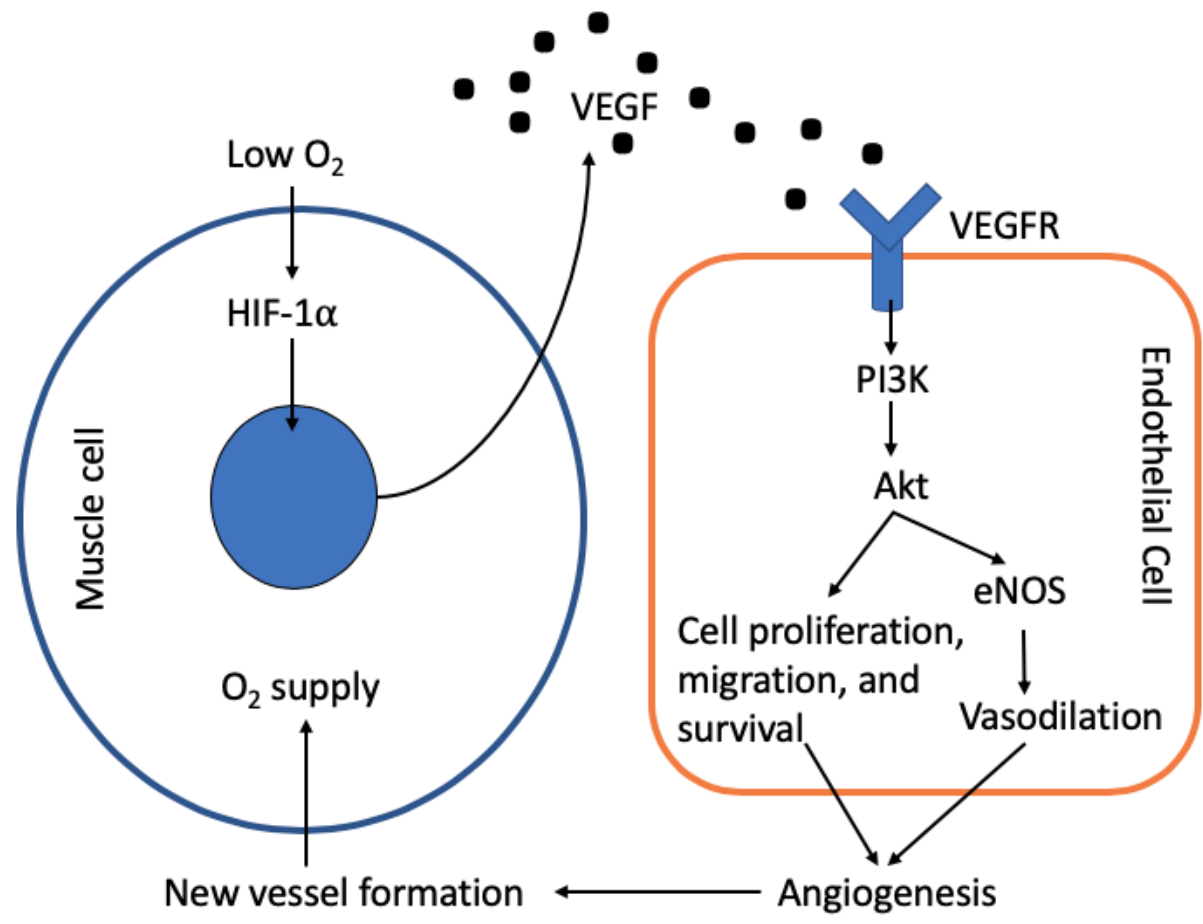

Figure 6: Hypoxia mediated angiogenesis via VEGF/Akt axis. Under hypoxic condition, VEGF production is up-regulated in tissue under hypoxic conditions via inducing hypoxia inducing factor $1 \alpha(\mathrm{HIF} 1 \alpha)$ and that plays a pivotal role in angiogenesis via activating multiple angiogenic signaling cascades for endothelial cell proliferation, migration, and survival.

An early study showed that Hcy treatment on chicken embryos (embryonic day 3.5) caused inhibition of early extraembryonic vascular development, altered 
composition of the vascular beds, and reduced VEGF-A and VEGFR-2 expression (200). Similarly, when HUVEC cells were treated with Hcy, it reduced cell proliferation and viability, induced a G1/S arrest, and attenuated the cell migration and tube-like formation on Matrigel (201). This study showed that this effect is mediated through VEGF/VEGFR, Akt, and ERK1/2 inhibition (201) as shown in Fig. 6. Similarly, a study demonstrated that Hcy inhibits angiogenesis by preventing proliferation and migration of ECs in a dosedependent manner (199). In an in-vivo rat model, it was reported that HHcy impaired ischemia-induced angiogenesis and collateral vessel formation, which is partly mediated through the reduction in bioactivity of endogenous NO (197). Several groups showed that Hcy-mediated defective angiogenesis is largely due to decreased GPx expression and a consequent increase in oxidative stress, leading to endothelial progenitor cell dysfunction $(203,204)$, reduction of the bioavailability of NO $(205,206)$ and dysregulation of matrix metalloproteinases (MMPs) $(207,208)$.

Previous studies showed that high Hcy can be toxic to endothelial cells and can induce endothelium dysfunction may be via hypomethylation of specific genes (209212). It also noticed that Hcy affects ECs via inhibiting cyclin A (213). When they transduced cyclin A in ECs, the inhibitory effect of Hcy was rescued. These studies have been conducted in an in-vitro system; however, there is much less evidence reported for the in-vivo system. As Akt has been associated to involved in ECs proliferation, migration and survival during angiogenesis and HHcy found to inhibit Akt activation in ECs, however, whether these two processes potentiate muscle atrophy is not studied yet $(37,45)$.

\subsection{HHcy-mediated skeletal muscle atrophy}

As mentioned earlier, skeletal muscle is the largest organ in our body, containing $50-75 \%$ of the body's proteins. Loss of skeletal muscle mass is commonly referred to as 
muscle atrophy. Several E3 ubiquitin ligases such as MuRF1, MAFBx (Atrogin-1), Nedd4.1, TRAF6, and MUSA1 have been identified to mediate proteolytic degradation of both thick and thin filament proteins in skeletal muscle atrophy $(214,215)$. Forkhead Box (FOX) proteins are a group of transcription factors with a conserved DNA binding domain and are responsible for regulating a number of E3-ubiquitin ligases upon a variety of cellular stress responses (216-219). Studies showed that in the absence of growth factors or in the presence of external stress (oxidative/ER-stress), PI3K/Akt pathway activation decreases and JNK activation increases; which promote nuclear localization of Foxo $(216,220-223)$ as shown in Fig. 7 . Although nuclear localization of FOXO stimulates MuRF-1 and Atrogin-1 expression, and that induces muscle atrophy (224), however, whether HHcy also causes skeletal muscle atrophy via this similar mechanism was not studied (Fig. 7).

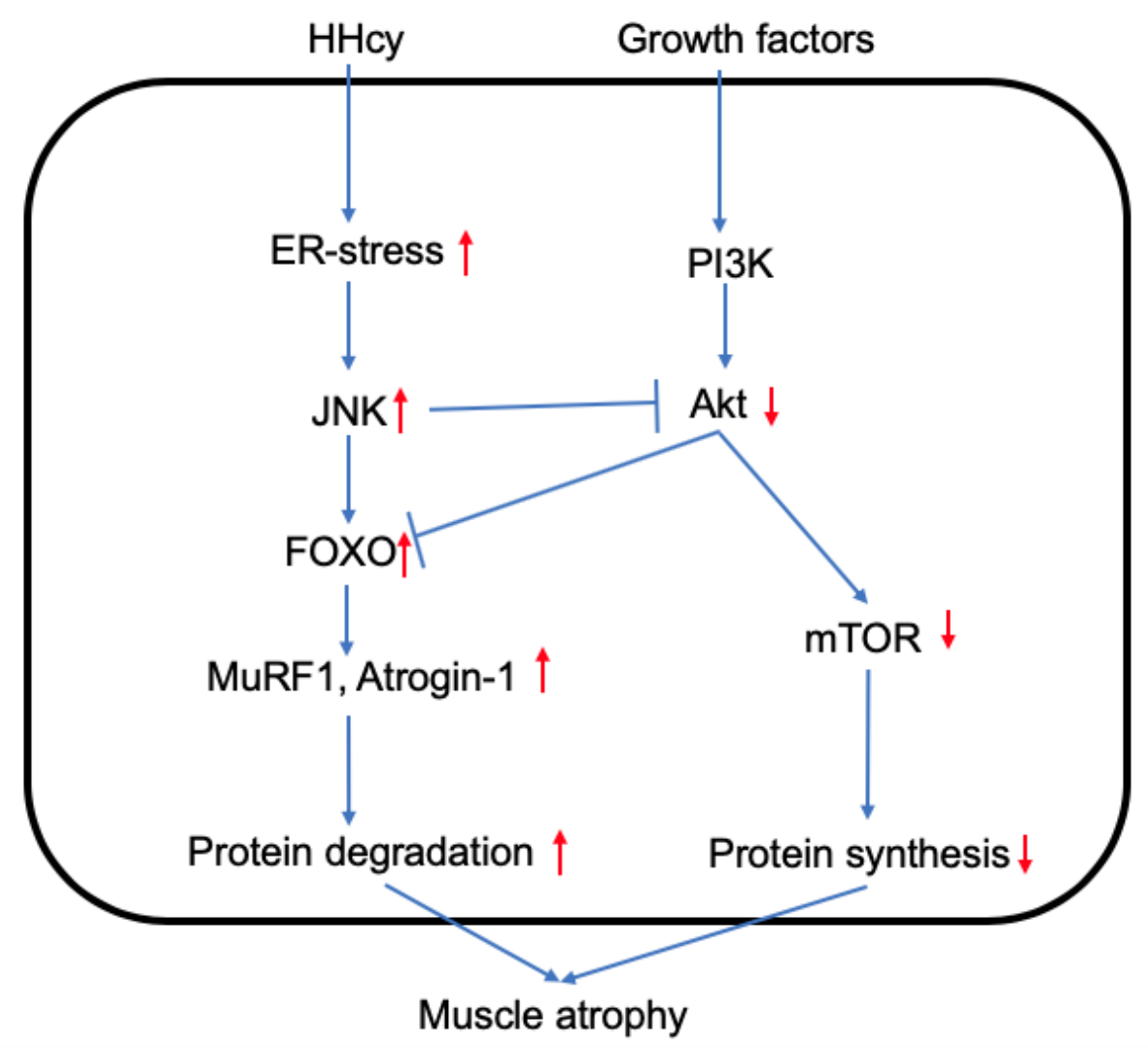

Figure 7: Schematic model of HHcy-induced muscle atrophy via nuclear translocation 
of FOXO. In HHcy condition, JNK activation may be upregulated and Akt-activation may be downregulated, which promotes nuclear localization of FOXO and reduced activation of mammalian target of rapamycin (mTOR). Nuclear localization promotes upregulation of MuRF-1 and Atrogin-1, which finally degrade muscle proteins. Whereas reduced activation of mTOR reduced protein synthesis. Muscle atrophy causes, when protein degradation exceeds over protein synthesis over time.

It has been reported that HHcy is associated with a decline in physical performance and skeletal muscle dysfunction in genetically engineered CBS deficient mice $\left(\mathrm{CBS}^{+-}\right)(5,20,82)$. Skeletal muscle dysfunction and poor physical performance can result from a reduction of muscle mass and/or structural and metabolic alteration (Fig. 7) $(139,225,226)$. Indeed, a study found that cystathionine $\gamma$-Lyase-deficient mice have lethal myopathy and oxidative injury, wherein dietary cysteine supplementation could mitigate this effect (84). Another group reported patients with HHcy due to methyltetrahydrofolate reductase (MTHFR) mutations (C677T and A1298C) showed poor survival and muscle pain in these patients (227). Similarly, a study from our group concluded that vasodilation in skeletal muscle arterioles is decreased during HHcy due to reduced expression of gap junction proteins such as connexins 37,40 and 43 , and increased expression of myostatin in the skeletal muscle (228).

\section{Effect of hydrogen sulfide on hyperhomocysteinemia-mediated skeletal muscle myopathy}

Hydrogen sulfide $\left(\mathrm{H}_{2} \mathrm{~S}\right)$ is increasingly being recognized as a novel endogenous gasotransmitter like nitric oxide (NO) and carbon monoxide (CO) $(54,59,229)$. Being a potent anti-apoptotic/anti-necrotic $(230,231)$, anti-inflammatory $(232)$ and cytoprotective agent (233), $\mathrm{H}_{2} \mathrm{~S}$ plays crucial roles in physiological homeostasis wherein it reduces 
oxidative damage $(51,234)$. As mentioned earlier, high SAM level can act as an allosteric inhibitor for MTHFR in the remethylation pathway (110) and allosteric activator for CBS in the transsulfuration pathway $(111,112)$. Together these results suggest that the presence of a high level of SAM favors Hcy entering into the transsulfuration pathway; however, in patients with CBS mutation, a high SAM level builds up (due to MET load) leading to transient accumulation of $\mathrm{Hcy}$ and reduction of $\mathrm{H}_{2} \mathrm{~S}$ levels. The endogenous $\mathrm{H}_{2} \mathrm{~S}$ production ranges from 1-10 pmoles per second per mg protein (235). Patients with CBS mutation have a high amount of Hcy and tend to produce a lesser amount of $\mathrm{H}_{2} \mathrm{~S}$ $(63,236)$, suggesting that these patients are likely more prone to oxidative stressmediated damage (54). Studies revealed that endogenous $\mathrm{H}_{2} \mathrm{~S}$ could modulate multiple signaling pathways, upregulate endogenous antioxidant systems and exert additive effects with known antioxidants (234), suggesting that exogenous supplementation of $\mathrm{H}_{2} \mathrm{~S}$ could be employed as a beneficial strategy to improve redox imbalance owing to HHcy (Fig. 8). 


\section{(A) Generation of endogenous $\mathrm{H}_{2} \mathrm{~S}$}

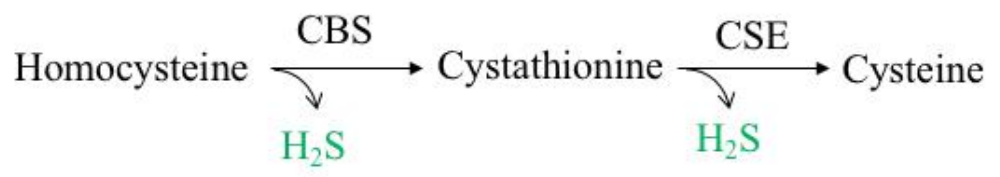

(B) $\mathrm{H}_{2} \mathrm{~S}$ serves as a scavenger of free superoxide radicals

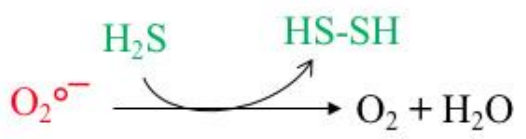

\section{(C) Reduction of proteins' disulfide bonds by $\mathrm{H}_{2} \mathrm{~S}$}

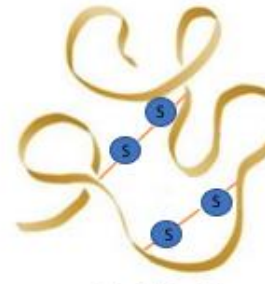

Oxidized

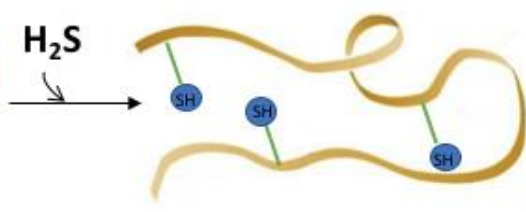

Reduced

Figure 8: Salubrious effects of $\mathrm{H}_{2} \mathrm{~S}$ on cellular biochemistry. (A) Being an important antioxidant molecule $\mathrm{H}_{2} \mathrm{~S}$ helps synthesize cysteine which gets converted into GSH via CBS and CSE enzymatic pathways. (B) $\mathrm{H}_{2} \mathrm{~S}$ is a potent scavenger for a variety of reactive oxygen species (ROS). (C) $\mathrm{H}_{2} \mathrm{~S}$ is known to reduce disulfide bonds in proteins and thereby it may reverse the homocysteinylation of proteins during HHcy conditions.

\subsection{The effect of Hydrogen sulfide on oxidative/ER-stress responses}

Multiple studies demonstrated the cytoprotective effects of $\mathrm{H}_{2} \mathrm{~S}$ in different in-vitro models, all relating to its ability to neutralize a variety of reactive species $(25,237,238)$ and reduction of a disulfide bond in proteins $(54,239)$ (Fig. 8). $\mathrm{H}_{2} \mathrm{~S}$ in water dissociates into $\mathrm{H}^{+}, \mathrm{HS}^{-}$, and $\mathrm{S}^{2-}$ ions. $\mathrm{HS}^{-}$has a capacity to scavenge $\mathrm{ROS} . \mathrm{H}_{2} \mathrm{~S}$ itself has also been recognized to be a reducing agent, as it can react directly with and quench the superoxide anion $\left(\mathrm{O}^{2-}\right)(240,241)$ and free radicals like peroxynitrite $(242)$ as well as 
other ROS in-vitro. Micro concentrations of $\mathrm{H}_{2} \mathrm{~S}$ generated from Na2S/NaHS was found to neutralize free oxyradicals (243), peroxynitrite (237), hypochlorous acid (238) and homocysteine (25) in in-vitro condition. There is no sulfide receptor in mammalian cells that is responsible for the biological actions of sulfide, hence sulfide, as a thiol with strong reducing activities, may also be a redox-controlling molecule similar to other small thiols, such as cysteine and GSH $(237,244)$. A study by Kimura et al. (2004) using primary cultures of neurons, found that $\mathrm{H}_{2} \mathrm{~S}$ increases the cellular GSH levels by enhancing the activity of gamma-glutamylcysteine synthetase and up-regulating cystine transport (244). Similarly, another study reported that $100 \mu \mathrm{M}$ NaHS induces glutamate uptake by assisting glial glutamate transporter-1 (GLT-1) and enhances cysteine transport and GSH synthesis (245). In support of this effect, multiple studies demonstrated that $\mathrm{H}_{2} \mathrm{~S}$ induces the cellular GSH in the brain (246), spinal cord (247), heart (248), lung (249), kidney (250), liver (249), and gastrointestinal tract $(251,252)$. Moreover, recent reports suggested that $\mathrm{H}_{2} \mathrm{~S}$ could attenuate cellular oxidative stress by improving activities of CAT (248,253-255) and GPx (256-258).

Many studies showed that $\mathrm{H}_{2} \mathrm{~S}$ attenuates ER-stress in numerous tissue types. A study found that $\mathrm{H}_{2} \mathrm{~S}$ attenuated HHcy-induced cardiomyocytic ER-stress in rats (259). Whereas, Li et al. (2015) demonstrated that $\mathrm{H}_{2} \mathrm{~S}$ protected against myocardial ischemia/reperfusion injury in rats by inhibiting ER-stress (260). Likewise, another study also reported that $\mathrm{H}_{2} \mathrm{~S}$ exerts its protection against the neurotoxicity of formaldehyde by overcoming ER-stress in PC12 cells (261).

\subsection{The effect of Hydrogen sulfide on cell death pathways}

In addition to an anti-oxidant effect of $\mathrm{H}_{2} \mathrm{~S}$, several studies also proposed that it has the ability to reduces cell death in various tissue types. An in-vitro study using nucleus pulposus (NP) from patients with lumbar disc herniation showed that exogenous 
$\mathrm{H}_{2} \mathrm{~S}$ donor attenuated hypoxia (generated by $\mathrm{CoCl}_{2}$ treatment) induced apoptosis in primary NP cells (262). Similarly, a study using a rat model found that $\mathrm{H}_{2} \mathrm{~S}$ mitigates cigarette smoke-induced apoptosis via reducing ER-stress (263). $\mathrm{H}_{2} \mathrm{~S}$ was also reported to decrease Hcy-mediated ER-stress and apoptosis in the hippocampus of rats by enhancing the BDNF-TrkB pathway (264). In support of this, NaHS supplementation was shown to significantly inhibit hypoxia-induced neuronal apoptosis through inhibition of $\mathrm{ROS}$ (mainly $\mathrm{H}_{2} \mathrm{O}_{2}$ )-activated $\mathrm{Ca}^{2+}$ signaling pathway in mouse hippocampal neurons (265). In another study, it was observed that $\mathrm{H}_{2} \mathrm{~S}$ attenuated gentamicin-induced apoptosis via reducing ROS moiety in normal rat kidney-52E cells; they found the protein levels of Bax, Caspase-3, and cleaved-caspase-3 were decreased while the expression of Bcl-2 was increased (266). Similarly, $\mathrm{NaHS}\left(\mathrm{H}_{2} \mathrm{~S}\right.$ donor) administration significantly inhibited the early phosphorylation of JNK and decreased the number of apoptotic cells, lowered cytochrome $\mathrm{C}$ release and enhanced $\mathrm{Bcl}-2$ expression in cardiomyocyte after ischemia-reperfusion (I/Re) injury (267). $\mathrm{H}_{2} \mathrm{~S}$ also attenuated 1methyl-4-phenylpyridinium ion (MPP (+))-induced apoptosis in PC12 cells by attenuating an overproduction of intracellular ROS (268). Exogenous $\mathrm{NaHS}\left(\mathrm{H}_{2} \mathrm{~S}\right.$ donor) also shown to ameliorate early brain injury after subarachnoid hemorrhage (SAH) by inhibiting neuronal apoptosis (268).

\subsection{The effects of Hydrogen sulfide on angiogenic defect}

It has been reported that $\mathrm{H}_{2} \mathrm{~S}$ is also involved in vasodilation, vascular protection, regulation of blood pressure and many other functions $(54,57-59,61)$. Like other gasotransmitters ( $\mathrm{CO}$ and $\mathrm{NO}$ ), $\mathrm{H}_{2} \mathrm{~S}$ can rapidly travel to vascular tissues without any transporter exerting a host of biological responses. Two independent groups reported using either transformed endothelial cell line (RF/6A) or HUVECs that $\mathrm{H}_{2} \mathrm{~S}$ induces proliferation and migration of these cells $(269,270)$. The pro-angiogenic effect of $\mathrm{H}_{2} \mathrm{~S}$ is 
further supported by the increase in tube-like structure formation in ECs in invitro Matrigel assays $(269,270)$. $\mathrm{H}_{2} \mathrm{~S}$ exerts angiogenic effects via releasing VEGF in both SMCs (smooth muscle cells) and ECs $(271,272)$. Some in-vivo studies demonstrated that $\mathrm{H}_{2} \mathrm{~S}$ induced a concentration-dependent increase in the length and complexity of the vascular network $(269,270)$. When ECs were exposed to $\mathrm{H}_{2} \mathrm{~S}$ donors, it activated multiple signaling pathways, such as PI-3K/Akt and MAPK pathways during neovascularization $(269,270)$. Further evidence for $\mathrm{H}_{2} \mathrm{~S}$-mediated VEGF induction during angiogenesis was reported in an ex-vivo mouse aorta sprouting assay, where the VEGFinduced angiogenesis was markedly suppressed in aortic rings of the CSE deficient mice $\left(\mathrm{CSE}^{--}\right)(270)$. In an alternative pathway, exposure to $\mathrm{H}_{2} \mathrm{~S}$ increased calcium levels in ECs, which led to eNOS activation and increased NO release during angiogenesis (273275).

\subsection{The effects of Hydrogen sulfide on muscle atrophy}

Skeletal muscles are now considered to be an endocrine organ, synthesizing and secreting a variety of bioactive molecules including inflammatory cytokines, growth factors, adipokines, carnitine and more recently $\mathrm{H}_{2} \mathrm{~S}$ (276). In this context, $\mathrm{H}_{2} \mathrm{~S}$ appears to protect against low oxygen and nutrient supply as well as ischemic injury in multiple organs including skeletal muscles (276-278). In agreement with this view, it was hypothesized that dysregulation of $\mathrm{H}_{2} \mathrm{~S}$ metabolism is involved in chronic fatigue syndrome (CFS), also called myalgic encephalomyelitis (279). One of the cellular targets of $\mathrm{H}_{2} \mathrm{~S}$ is potassium ( $\left.\mathrm{K}_{\text {ATP }}\right)$ channel $(280,281)$. $\mathrm{K}_{\text {ATP }}$ channel activation has been suggested to be involved in the prevention of calcium overload and preservation of myofibre integrity during exercise, recovery from muscle fatigue, rather than in normal muscle contractility and excitability $(282,283)$. Similarly, a significant decrease in $\mathrm{H}_{2} \mathrm{~S}$ content in muscle fibers together with a reduction in SOD1 expression was presented in 
a rat model of skeletal muscle ischemia-reperfusion (I-R) injury (276). 


\section{CHAPTER II \\ HYPOTHESIS AND SPECIFIC AIMS}

Key Objective

The objective of this study was to determine the effects of

hyperhomocysteinemia on skeletal muscle myopathy and investigate if $\mathrm{H}_{2} \mathrm{~S}$ can attenuate these effects.

Hypothesis

The central hypothesis of this proposal is that HHcy causes skeletal muscle myopathy by inducing apoptosis, inflicting muscle atrophy and compromising angiogenesis via JNK-activation and concomitant impairment of PI3K/AKT axis, and $\mathrm{H}_{2} \mathrm{~S}$ mitigates these effects (Fig. 9).

Specific Aim\#1: To determine whether HHcy induces ER-stress and apoptosis via JNKphosphorylation, effect(s) of $\mathrm{H}_{2} \mathrm{~S}$ treatment in mitigating this induction.

Specific Aim\#2: To determine whether the Hcy-activated JNK downregulates angiogenic pathways via inhibiting AKT-phosphorylation and $\mathrm{H}_{2} \mathrm{~S}$ treatment ameliorates these pathway(s).

Specific Aim\#3: To determine whether Hcy instigates muscle atrophy via up-regulation of E3 ubiquitinligases through JNK/AKT axis and $\mathrm{H}_{2} \mathrm{~S}$ attenuates muscle atrophy 


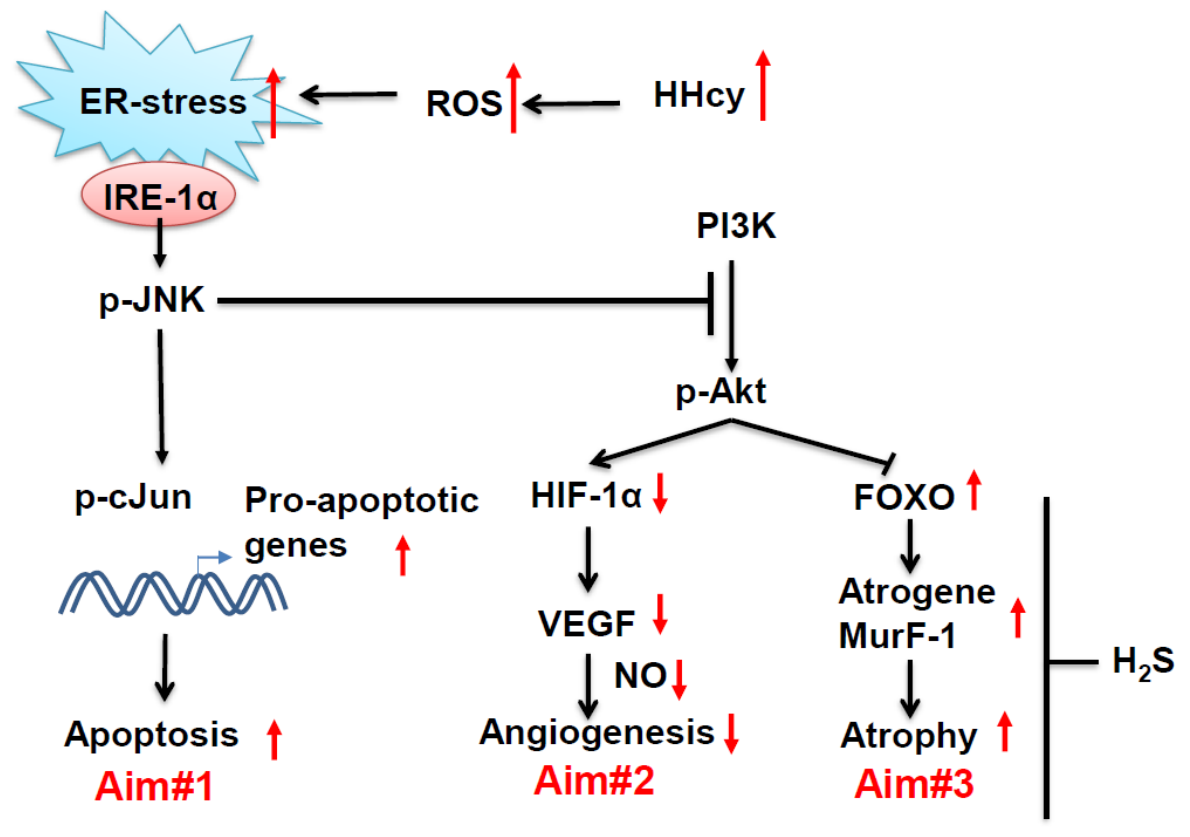

Figure 9: A basic model of my hypothesis 


\section{CHAPTER III}

HYDROGEN SULFIDE ALLEVIATES HHCY-MEDIATED OXIDATIVE AND ER-STRESS

RESPONSES IN SKELETAL MUSCLE ${ }^{(284)}$

\section{INTRODUCTION}

Homocysteine (Hcy) is a sulfur-containing non-proteinogenic amino acid that is generated during methionine metabolism via the methionine cycle (MET-cycle) (285). In healthy subjects, synthesis and elimination of Hcy are balanced; however, if Hcy metabolism is disturbed, then its plasma levels are elevated, leading to hyperhomocysteinemia (HHcy) $(20,82-84,286)$. Although children born with functional CBS deficiency show severe HHcy and die in their teenage years $(13,15,20)$, the molecular mechanism(s) how HHcy triggers such pathological effects in skeletal muscle is not fully understood.

Previous studies revealed that Hcy contains a -SH group like thiols $(\mathrm{RSH})$ which can undergo oxidation to form a disulfide (RSSR) even at physiological $\mathrm{pH}$ in the presence of metal catalysts and molecular oxygen $\left[\mathrm{O}_{2}{ }^{\circ}\right](21)$. Further, Hcy can also produce hydrogen peroxide $\left(\mathrm{H}_{2} \mathrm{O}_{2}\right.$, a pro-oxidant molecule) during metal-catalyzed oxidation step and peroxynitrite ( $\mathrm{ONOO}^{-}$, a powerful oxidant) in the presence of nitric oxide (NO) and superoxide anion $\left(\mathrm{O}_{2}^{-}\right)(144)$. Although these phenomena have been studied in multiple tissue types, whether HHcy exerts its detrimental effects on muscle through these mechanisms is not yet elucidated. Oxidative stress has been implicated in many diseases associated with protein misfolding (29-31). Likewise, studies have also reported that HHcy could induce ER-stress in hepatocytes as well as in vascular 
endothelial and aortic smooth muscle cells, but the cellular pathways that are involved in these stress-related conditions are not adequately studied $(32,33)$. It is well-known that after translation, the protein folding occurs inside the ER, but during stress conditions, misfolded proteins can accumulate inside ER-lumen inducing the unfolded protein response (UPR) (152). In mammals, there are three branches of UPR: inositol-requiring enzyme-1 (IRE1); PRKR-like ER kinase (PERK); and activating transcription factor6 (ATF6) $(153,154,156)$. During severe ER-stress conditions, activated IRE1 $\alpha$ recruits' TNF receptor-associated factor-2 (TRAF2) and apoptosis signal-regulating kinase-1 (ASK1), which further activate c-Jun N-terminal kinase (JNK) (34-36). Activation of JNK phosphorylates c-Jun at Ser63 and 73 residues in $\mathrm{NH}_{2}$-terminal $(287,288)$. Upon activation, the phosphorylated JNK translocate to the nucleus where it phosphorylates cJun $(289,290)$. JNK along with c-Jun makes up the activator protein-1 (AP-1) transcription factor, which involved in the transcription of some pro-apoptotic and proinflammatory genes (291-296). JNK also regulates maturation and activity of T cells in addition to the synthesis of pro-inflammatory cytokines, such as interleukin-2 (IL-2), IL-6 and TNF- $\alpha$ (297-299). Whether HHcy can compromise muscle survival via JNK activation is not currently known.

Chronic systemic inflammation and apoptosis are the important driver for muscle wasting, that can be dysregulated by HHcy condition (300). These cytokines work synergistically promoting muscle atrophy due to the cross-talk between inflammatory cells and organs, resulting in reduced protein synthesis and increased protein degradation, and ultimately leading to muscle loss and functional impairment. Therefore, identification of the precise molecular mechanism(s) as to how these cytokines are regulated during HHcy is essential to devise future preventive strategies.

$\mathrm{H}_{2} \mathrm{~S}$ is increasingly being recognized as an important signaling molecule in the cardiovascular and nervous systems via its ability to neutralize a variety of reactive 
oxygen species (ROS) $(25,237,238)$ and to reduce disulfide bonds $(54,239)$.

Cystathionine $\mathrm{y}$-lyase (CSE) and CBS can irreversibly remove Hcy by converting it into $\mathrm{H}_{2} \mathrm{~S}$, since patients with CBS lack $\mathrm{H}_{2} \mathrm{~S}$, making them vulnerable to oxidative stressmediated damages (54). Hence, the purpose of my study was to understand the effect(s) of HHcy-mediated oxidative and ER-stress responses in muscle and the beneficial effects of an $\mathrm{H}_{2} \mathrm{~S}$ donor ( $\mathrm{NaHS}$ ) employing both in-vitro ( $\mathrm{C} 2 \mathrm{C} 12$ cells) and in-vivo model (CBS+/-) systems. My results indicate that $\mathrm{H}_{2} \mathrm{~S}$ could be developed as a potential therapeutic target in various forms of musclulopathies wherein HHcy is linked with metabolic dysfunction.

\section{MATERIALS AND METHODS}

Animal maintenance and diet protocol. Male wild-type (WT, C57BL/6J) and $\mathrm{CBS}^{+/-}$(B6.129P2-Cbstm1Unc/J 002853) mice were purchased from the Jackson Laboratory (Bar Harbor, ME) (71). All animals were 8-12 weeks-old and maintained in $12: 12 \mathrm{~h}$ light-dark cycle with regular mouse chow diet in the animal facility of the University of Louisville. All animal protocols and care were carried out according to the guidelines of National Institute of Health (NIH Pub. No. 86-23, revised 1985) and were approved by the Institutional Animal Care and Use Committee (IACUC) of the University of Louisville. Animals were divided into 4 experimental groups: (1) Wild-type C57BJ/L6 mice (WT); (2) $\mathrm{CBS}^{+/-}$heterozygous mice fed with methionine (CBS+Met); (3) NaHS-supplemented wild-type mice (WT+NaHS); (4) NaHS-supplemented $\mathrm{CBS}^{+/-}+$Met mice (CBS+Met+NaHS). Mice were treated with $\mathrm{NaHS}$ for 8 weeks $(30 \mu \mathrm{M} / \mathrm{kg} / \mathrm{day}, \mathrm{I} . \mathrm{P}$.) and fed with a methionine-enriched and low-folate, low Vitamin B6, and low Vitamin B12 diet (Cat. No. TD 97345; Harlan Teklad, Madison, WI, USA) (301-304). WT mice were given $0.9 \%$ normal saline (vehicle control) and fed with normal chow (Purina Farmer's Exchange, Framingham, MA, USA). 
Rationale for choosing the experimental mice. Homozygous for CBS knock out (KO) mice $\left(\mathrm{CBS}^{-/}\right)$mice die shortly after their birth, whereas heterozygous for CBS KO mice $\left(\mathrm{CBS}^{+/}\right)$survive but they show low Hcy levels in their blood (5-10 $\mu \mathrm{M}$, Fig. 15D). So, in the present study, I treated $\mathrm{CBS}^{+/-}$mice with high met diet to examine the effects of HHcy condition (>40 $\mu \mathrm{M}$, Fig. 16F) on skeletal muscle (301-304). Genotyping analysis of the heterozygous CBS+/- mouse. After purchasing, mice were cross-bred, yielding around $10 \% \mathrm{CBS}^{-/-}, 60 \% \mathrm{CBS}^{+/-}$, and $25 \% \mathrm{CBS}^{+/+}$. For genotyping, tail samples were collected, genotypic analysis was performed using PCR by targeted disruption of the CBS gene. The PCR products were run on $1.2 \%$ agarose gel (prepared in TAE buffer, $\mathrm{pH}$ 8.4) in the presence of ethidium bromide and the images recorded in a gel documentation system (305). $\mathrm{CBS}^{+/-}$heterozygote gene-positive mice produced two bands (450 and $308 \mathrm{bp}$ ), while $\mathrm{CBS}^{+/+}$(WT) mice represented only one band (308 bp).

Reagents and antibodies. Dulbecco's Modified Eagle's Medium (DMEM), fetal bovine serum (FBS) were purchased from American Type Culture Collection (Manassas, VA, USA) and trypsin EDTA was from VWR (Radnor, PA, USA). ECL reagent and polyvinylidenedifluoride (PVDF) membrane were from Bio-Rad (Hercules, CA, USA). Dihydroethidium (DHE) was purchased from Thermo Fisher Scientific (Waltham, MA, USA). All other reagents and chemicals were ordered from Sigma-Aldrich or available highest grade.

The antibodies for GRP78 (Cat. No.\# sc-13968), IRE1a (Cat. No.\# sc-20790), ATF6 (Cat. No.\# sc-22799), X-box binding protein (XBP1) (Cat. No.\# sc-7160), rabbit anti-mouse (Cat. No.\# sc-358914), mouse anti-rabbit (Cat. No.\# sc-2357), and mouse anti-goat (Cat. No.\# sc-2354) were ordered from Santa Cruz Biotechnology (Dallas, TX, U.S.A). The antibody for GAPDH (Cat. No.\# MAB374) was ordered from EMD Millipore (Burlington, MA, USA). Rest of the antibodies for p-IRE1a (S724) (Cat. No.\# ab48187), 
p-JNK (T183/Y185) (Cat. No.\# ab4821), t-JNK (Cat. No.\# ab85139), p-cJun (S73) (Cat. No.\# ab30620), and t-cJun (Cat. No.\# ab32137) were ordered from Abcam (Cambridge, MA, USA), and used for Western blots analysis as per the manufacturers' protocols. Cell culture and treatments. The $\mathrm{C} 2 \mathrm{C} 12$ cells (immortalized mouse myoblast cell line, ATCC) were cultured in Corning® T-75 flasks in ATCC-formulated DMEM (catalog No. 30-2002) supplemented with $10 \% \mathrm{FBS}, 0.1 \%$ of penicillin and streptomycin at $37^{\circ} \mathrm{C}$ with $5 \% \mathrm{CO}_{2}$. The $\mathrm{C} 2 \mathrm{C} 12$ cells were grown to $80 \%$ confluence and were plated for 4 different experimental groups: Group 1: CT (PBS as vehicle control); Group 2: Hcy (1mM); Group 3: Hcy+NaHS $(250 \mu M)$; and Group 4: NaHS. In this study, we used 1mM Hcy and $250 \mu \mathrm{M}$ NaHS concentrations for the individual treatments $(20,246)$. A stock solution of Hcy, NaHS were prepared by directly dissolving in basal DMEM medium (serum-free media). Following $24 \mathrm{~h}$ of treatment as mentioned earlier, cells were processed for qPCR, Western blot, DHE-staining, and other biochemical analysis.

Total RNA extraction. Total RNA was extracted from muscle samples and cells using a Trizol method (306). In brief, first equal volume of Trizol was added in the sample and incubated for $10 \mathrm{mins}$ in $25^{\circ} \mathrm{C}$. Then $200 \mu \mathrm{l}$ of chloroform was added and incubated for $10 \mathrm{mins}$ on ice. After that, samples were centrifuged at $12000 \mathrm{~g}$ for $15 \mathrm{mins}$ at $4^{\circ} \mathrm{C}$ and carefully collected the top layer in a new Eppendorf tube containing 500uL of prechilled isopropanol. Then samples were centrifuged at $12000 \mathrm{~g}$ for $20 \mathrm{mins}$ at $4^{\circ} \mathrm{C}$ and pellets were collected in DEPC water. Total RNA quality was determined by NanoDrop ND1000, and RNA with high purity (260/280 2.00 and 260/230 2.00) was used for q-PCR. Reverse transcription and real-time quantitative PCR (RT-qPCR). Reverse transcription was performed according to the manufacturer's protocol using high-capacity cDNA reverse transcription kit from Applied Biosystems (Foster City, CA, USA) for the primer sequences listed in Table 2. For RT-qPCR, SYBR green-based kit was used to measure the relative expression of each mRNA specific primers. Briefly, 3 steps cycling protocol 
was performed using $20 \mathrm{ng}$ of cDNA template in a $20 \mu \mathrm{L}$ reaction volume under the following conditions: denaturation at $95^{\circ} \mathrm{C}$ for $15 \mathrm{~min}$ followed by 40 cycles of $94^{\circ} \mathrm{C}$ for $15 \mathrm{~s}, 55^{\circ} \mathrm{C}$ for $30 \mathrm{~s}$, and $70{ }^{\circ} \mathrm{C}$ for $34 \mathrm{~s}$ in which fluorescence was acquired and detected by Roche LightCycler® 96 Real-Time PCR System (Roche Diagnostics, IN, USA). Following RT-qPCR, analysis of melt curve was performed to validate the specific generation of the expected PCR product. GAPDH was used as an endogenous control (Quanta Biosciences, Beverly, MA, USA).

\begin{tabular}{|c|c|c|}
\hline Genes & Forward Primers & Reverse Primers \\
\hline GRP78 & 5'-ATTGGTGGCCGTTAAGAATG-3' & 5'-CAGTGTTGTCTCGGCCAGTA-3' \\
\hline ERN1 & 5'-CCCAAATGTGATCCGCTACT-3' & 5'-TTGAGAGAATGCAGGTGTGC-3' \\
\hline ATF6 & 5'-GGCCAGACTGTTTTGCTCTC-3' & 5'-CCCATACTTCTGGTGGCACT-3' \\
\hline XBP1 & 5'-TGAATGGCCCTTAGCATTTC-3' & 5'-CACAGAACAGGACGCTGTGT-3 \\
\hline
\end{tabular}

Table 2. List of primers used for RT-qPCR experiments for chapter IV.

Western blotting. All protein expressions of both tissues and cells were assessed by Western blotting (307). Briefly, all protein lysates were made in RIPA buffer, which was supplemented with PMSF (1 mM), Na-orthovanadate $(1 \mathrm{mM})$ and a protease inhibitor cocktail (10 $\mu \mathrm{l} / \mathrm{ml}$ of lysis buffer). The protein samples were estimated by Bradford assay. Equal amounts of protein $(50 \mu \mathrm{g})$ were resolved on SDS-PAGE $(8 \%, 10 \%, 12 \%)$ and transferred onto a polyvinylidene difluoride (PVDF) membrane. The blots were visualized using ECL Luminata Forte (Millipore, Temecula, CA, USA) in a Bio-Rad ChemiDoc system. The band intensity was normalized to GAPDH for all the proteins and quantified using the Bio-Rad's Image Lab software (Bio-Rad, Hercules, CA, USA). 
Total Homocysteine measurement. The total thcy levels were measured from plasma of experimental mice using homocysteine assay kit (Crystal Chem, IL, USA) as per manufacturer's instructions.

Intracellular ROS imaging by confocal scanning microscopy. The cell permeable fluorescent dye dihydroethidium (DHE) was used to detect intracellular ROS (72). In brief, after treatment $\mathrm{C} 2 \mathrm{C} 12$ cells were incubated in DHE $(10 \mu \mathrm{M} / \mathrm{L})$ for 20 minutes in a humidified chamber at room temperature in the dark. At the end of the incubation, cells were washed with PBS and fluorescence images were scanned using a laser confocal microscope (Olympus FluoView1000, Pittsburgh, PA, USA) (72). Fluorescent intensity was quantified using ImageJ software (https://imagej.nih.gov/ii)). Assessment of lipid peroxidation. Malondialdehyde (MDA), a metabolite of lipid peroxidation and an indicator of oxidative stress was measured as the previously described method by Okhawa et al. (308). Briefly, after treatment, the cells were washed with PBS, followed by sonication (5 Amps/ 5 mins) and centrifugation at $13000 \mathrm{rpm}$ for 10 minutes. To the supernatant $100 \mu \mathrm{l}$ of $8.1 \%$ SDS, $20 \%$ acetic acid, and $0.8 \%$ thiobarbituric acid were added. The samples were incubated at $95^{\circ} \mathrm{C}$ for 60 minutes, and then $100 \mu$ l of deionized $\mathrm{H}_{2} \mathrm{O}$ and $100 \mu$ l of 1 -butanol were added. After spinning at 4000 rpm for 10 minutes, the top layer was collected in 96 wells plate and read at a wavelength of $532 \mathrm{~nm}$. The OD values were plotted against the known standard to determine the intracellular malondialdehyde levels.

Assessment of hydrogen peroxide and total ROS. $\mathrm{H}_{2} \mathrm{O}_{2}$ was measured from cells by Amplex red assay kit (Invitrogen, Cat no. A22188) according to the manufacturer protocol. Whereas, 2', 7'-dichlorofluorescein diacetate (2',7'-DCFDA) assay kit (Abcam, Cat no.-ab113851) was used for total cellular ROS detection. In brief, after treatment cells were washed two times with PBS and incubated with a $10 \mu \mathrm{M}$ DCFDA probe for 15 mins in serum-free media. Final fluorescence intensity was measured in a microplate 
reader $(E x, E m=485,535 n m)$.

Measurement of GSH/GSSG ratio. Assessment of GSH vs. GSSG ratio was carried out in all 4 treatment groups using GSH/GSSG ratio detection kit (Abcam, Cat no.-

ab138881) according to the manufacturer protocol.

Assessment of pro-inflammatory cytokines. Multi-analyte enzyme-linked immunosorbent assay (ELISA) kit (Qiagen, Germantown, MD, USA) was used following the

manufacturer's protocol to measure all the pro-inflammatory cytokines (IL1 $\alpha$, IL2, IL4, IL6, IL12A, IFN- $\gamma$, and TNF- $\alpha$ ).

Cell viability assay. Cell viability was determined by MTT assay (309). In brief, after treatment as described above, all media was aspirated and $100 \mu \mathrm{l}$ MTT [3-(4,5dimethylthiazol-2-yl)-2,5-diphenyltetrazolium bromide] reagent solution was added in each well after diluting MTT reagent $(5 \mathrm{mg} / \mathrm{mL})$ in basal media. The cells were then incubated for $4 \mathrm{~h}$ at $37^{\circ} \mathrm{C}$ and then washed with PBS. After that, $100 \mu \mathrm{l}$ of DMSO was added to each well to dissolve the cell membrane and bring formazan into the solution. The absorbance values were taken at $570 \mathrm{~nm}$ by Spectramax 3000 spectrophotometer (Molecular Devices, Sunnyvale, CA, USA).

Flow cytometry. C2C12 cells were grown in 12 well plates until $70 \%$ confluence and treated as mentioned above. After $48 \mathrm{~h}$, the cells were harvested with $150 \mu \mathrm{l}$ trypsinEDTA (Corning) and $600 \mu$ I DMEM complete medium to neutralize the trypsin. The cells were collected in a $1.5 \mathrm{ml}$ Eppendorf tube and spun at $2000 \mathrm{rpm}$ for 5 minutes, and the supernatant was discarded. Then cells were washed 2 times with complete DMEM media to remove any traces of trypsin and then resuspended in $1 \mathrm{x}$ binding buffer (Sigma apoptosis kit: Cat \# APOAF). The cells were then stained with Annexin V and Propidium lodide (PI) according to manufacturer's instructions and subjected to flow cytometric analysis (Accuri cytometers Inc. Ann Arbor, MI, USA).

Statistics. All values are expressed as mean \pm s.e.m. The overall difference between the 
experimental groups was determined by one-way or two-way ANOVA, and a Tukey's post hoc analysis was run when overall statistically significant difference occurred in group means. Whereas, unpaired t-test was used for comparison between two groups. The threshold for significance was set at $p<0.05$, and a minimum of 3 biological replicates were used for each experiment. For all statistical calculation, GraphPad Prism (Ver 7, GraphPad Software) was used.

\section{RESULTS}

Hcy induces oxidative stress in C2C12 cells. DHE-staining results showed that Hcy significantly induced ROS in $\mathrm{C} 2 \mathrm{C} 12$ cells as compared to vehicle controls (PBS treated), whereas, NaHS treatment mitigated the effects of Hcy (Fig. 10A). To detect intracellular total ROS levels, I used DCFDA fluorescent probe (an indicator for superoxide-, peroxide- and peroxynitrite-mediated oxidative chemistry). A similar finding was also observed in the levels of total ROS in Hcy treated cells compared to controls as measured by a fluorometric method (Fig. 10B). Hcy treatment showed significant induction of $\mathrm{H}_{2} \mathrm{O}_{2}$ production in $\mathrm{C} 2 \mathrm{C} 12$ cells, and it was reduced by $\mathrm{NaHS}$ (Fig. 10C). Similarly, malondialdehyde levels (a marker of lipid peroxidation) was significantly increased upon Hcy treatment in $\mathrm{C} 2 \mathrm{C} 12$ cells, and NaHS reversed this effect (Fig. 10D). Furthermore, a significant reduction in GSH/GSSG ratios in Hcy treated C2C12 cells as compared to controls, whereas NaHS treatment was found to successfully attenuate this effect (Fig. 10E). 

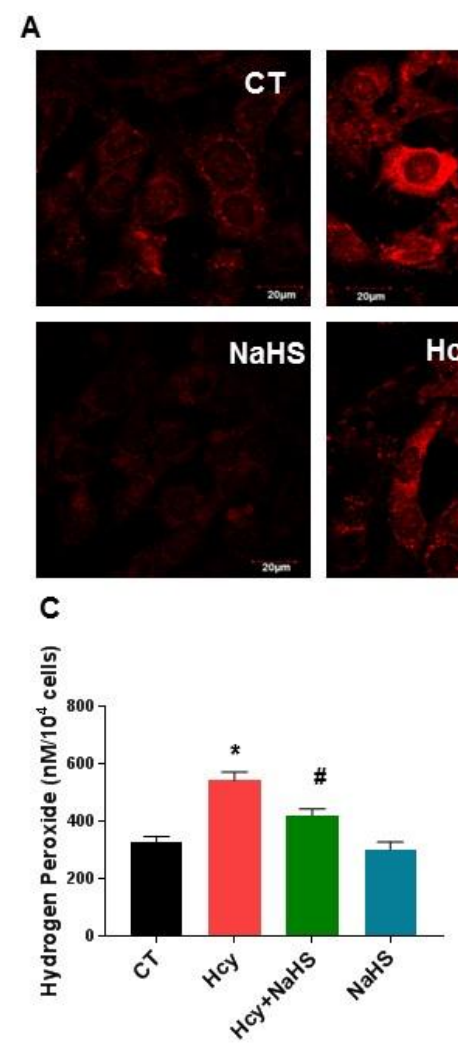

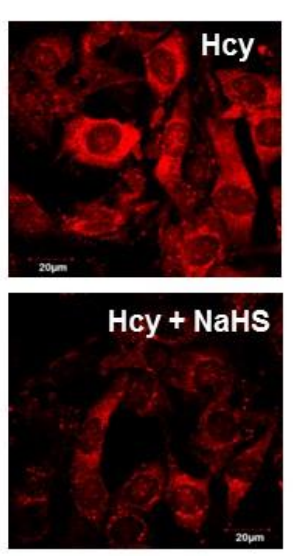

D

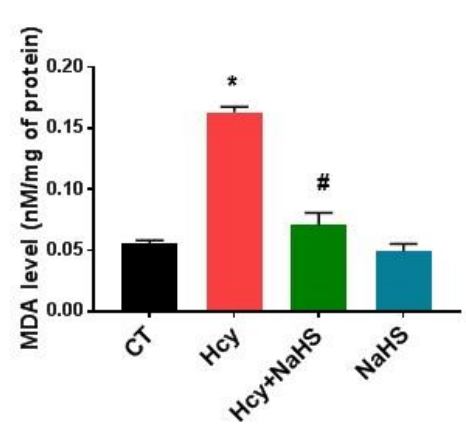

B
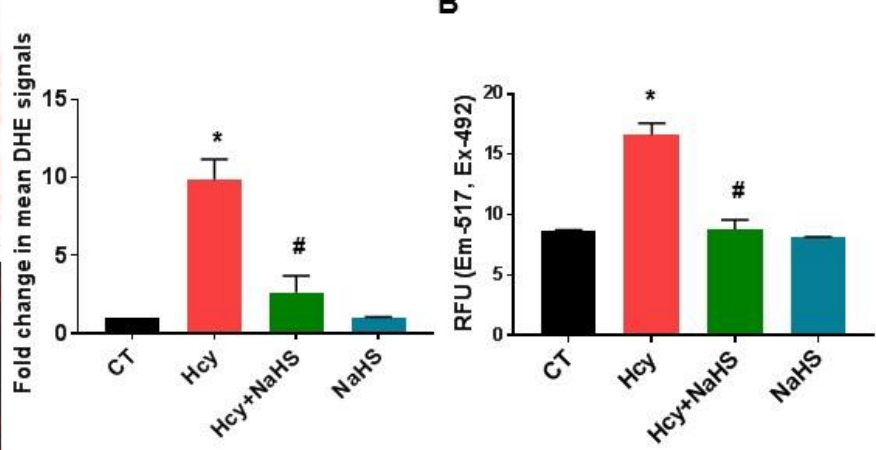

E

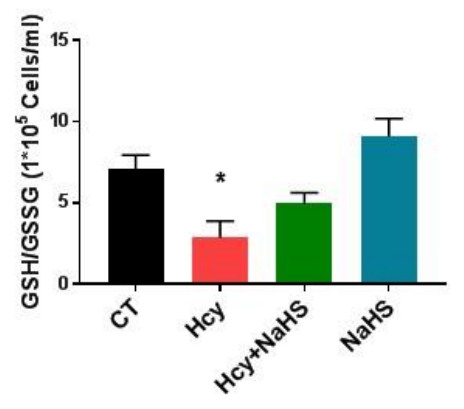

Figure 10: NaHS protects against Hcy-induced oxidative stress in C2C12 cells (immortalized mouse myoblast cell line). (A) $1 \mathrm{mM} \mathrm{Hcy}$ treatment for $24 \mathrm{~h}$ resulted in the generation of cellular oxidative stress in $\mathrm{C} 2 \mathrm{C} 12$ cells as detected by dihydroethidium (DHE)-staining. Images captured using confocal microscope are on the left and their quantification on the right (under $60 x$, bar=20 $\mu m$ ), where $n=3$ technical replicates; $(B)$ Hcy treatment induces total ROS levels as detected by 2', $7^{\prime}-\mathrm{H}_{2}$ DCFDA (2',7' dichlorofluorescin diacetate) probe, where $n=4$ technical replicates; (C) Hcy exposure induces $\mathrm{H}_{2} \mathrm{O}_{2}$ production measured by Amplex red assay kit, $\mathrm{n}=3$ technical replicates; (D) Hcy treatment induces lipid peroxidation measured by Malondialdehyde (MDA) assay, where $n=3$ technical replicates; (E) Hcy treatment reduces GSH vs. GSSG ratio, where $n=3-4$ technical replicates. The overall difference between the experimental groups was determined by one-way, and a Tukey's post hoc analysis was run when 
overall statistically significant difference occurred in group means. Data are shown as mean \pm s.e.m. and ${ }^{*} p<0.05$ vs. CT and ${ }^{\#} p<0.05$ vs. Hcy.

HHcy induces ER-stress responses in skeletal muscle. To examine whether HHcy induces ER-stress was via redox imbalance mechanism, I performed Western blot analyses on samples from both in-vitro and in-vivo models. Results showed that Hcy significantly induced ER-stress markers such as GRP78, ATF6 and p-IRE1 $\alpha$ and that the effect was mitigated by NaHS in C2C12 cells as shown in Fig. 11A and 11B. However, I did not notice any significant changes in XBP1 protein levels after Hcy treatment (Fig. $11 \mathrm{~A}$ and $11 \mathrm{~B})$. In addition, the ratios of $\mathrm{p}-\mathrm{IRE} 1 \alpha / \mathrm{t}-\mathrm{IRE} 1 \alpha$ were found to be significantly induced in $\mathrm{C} 2 \mathrm{C} 12$ cells after Hcy treatment compared to controls, whereas NaHS supplementation attenuated this effect (Fig. 11C). In the qPCR analysis, I noticed mRNA levels of GRP78, ATF6, IRE1 $\alpha$, and XBP1 were also increased significantly in post Hcy treatment of $\mathrm{C} 2 \mathrm{C} 12$ cells compared with vehicle controls, these effects were also mitigated by NaHS (Fig. 11D). In addition, my in-vivo model revealed similar type of changes in Western blots and qPCR data from the protein samples collected from hindlimbs gastrocnemius muscle (Fig. 11E-H). 

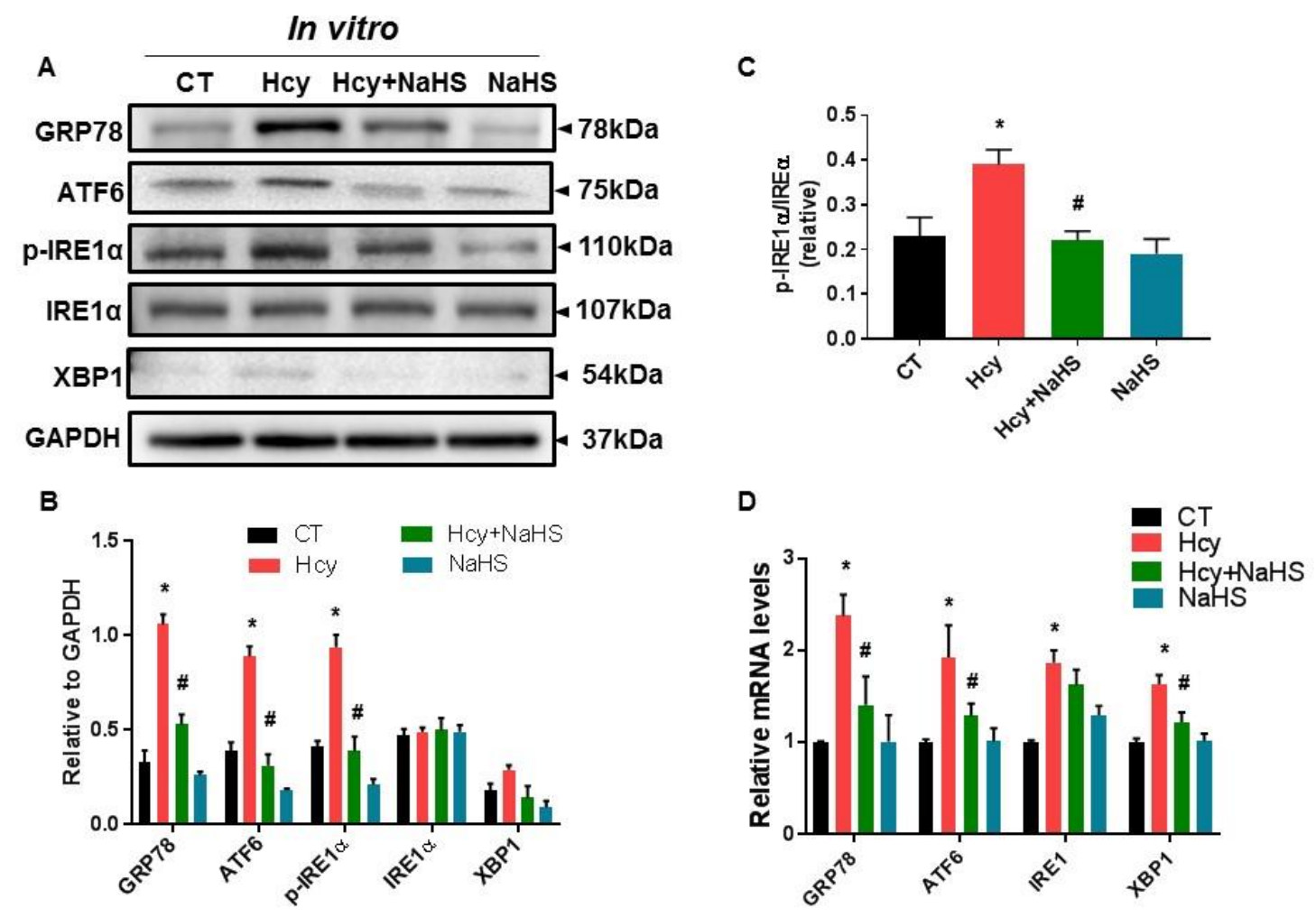

Figure 11A-D: High Hcy mediates ER-stress response in muscle cells in-vitro. (A) Western blots analysis showing Hcy treatment induces ER-stress markers, such as GRP78, ATF6, p-IRE1 $\alpha$ (S724), t-IRE $\alpha$ and XBP1, whereas NaHS treatment mitigates this effect, where $n=3$ technical replicates; (B) Densitometric measurement of ER-stress markers from above Western blots images are shown in the bottom, where $n=3$ technical replicates; (C) Quantification of p-IRE1 $\alpha / t-I R E \alpha$ ratio from Western blots images are shown on the right, where $n=3$ technical replicates; (D) Hcy induced expression mRNA levels of GRP78, ATF6, IRE1 $\alpha$ and XBP1 in C2C12 cells (log transformed), where $n=3$ technical replicates. The overall difference between the experimental groups was determined by one-way or two-way ANOVA, and a Tukey's post hoc analysis was run when overall statistically significant difference occurred in group means. Data are shown as mean \pm s.e.m, and ${ }^{*} p<0.05$ vs. CT and ${ }^{*} p<0.05$ vs. Hcy. 


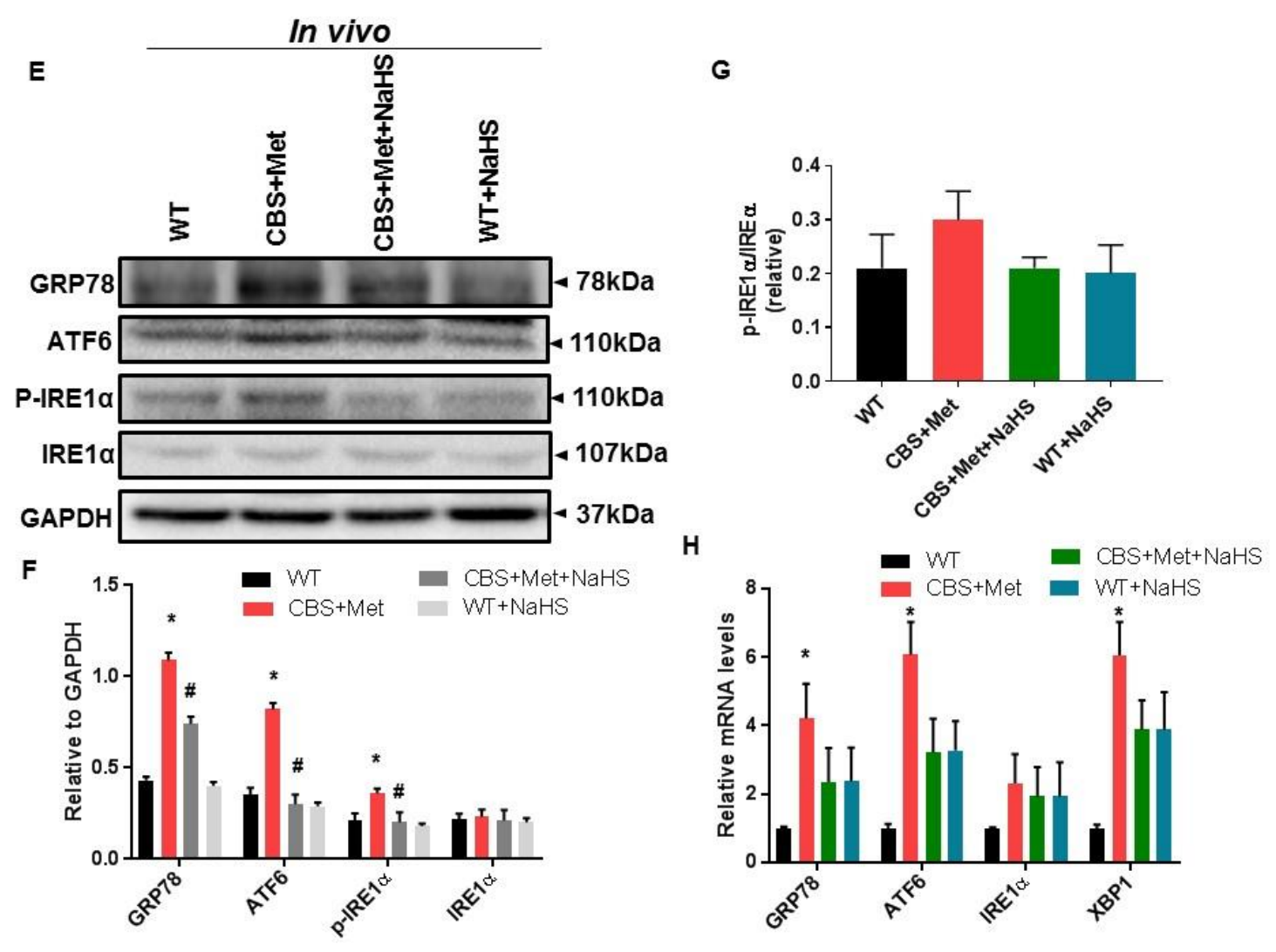

Figure 11E-H: High Hcy mediates ER-stress response in muscle in-vivo. (E) Western blots analysis showing induced expression of ER-stress markers, such as GRP78, ATF6, and p-IRE1 $\alpha$ (S724), t-IREa in CBS+Met mice compared to WT mice; whereas NaHS treatment mitigates this effect, where mice number $(n)=4$ in individual group; $(F)$ Densitometric quantification of Western blots of above ER-stress markers shown on the bottom, where mice number $(n)=4$ in individual group; $(G)$ Densitometric analysis of pIRE1a/t-IREa ratio from Western blots is shown on the right, where mice number $(n)=4$ in individual group; $(\mathrm{H})$ The q-PCR analysis shows that mRNA levels GRP78, ATF6, IRE1a, and XBP1 (log-transformed data) were increased in skeletal muscle of CBS+Met mice compared to WT mice, where mice number $(n)=4$ in individual group. The overall difference between the experimental groups was determined by one-way or two-way ANOVA, and a Tukey's post hoc analysis was run when overall statistically significant 
difference occurred in group means. Data are shown as mean \pm s.e.m and ${ }^{*} \mathrm{p}<0.05$ vs. WT and ${ }^{\#} p<0.05$ vs. CBS+Met.

Hcy enhanced JNK-phosphorylation. To confirm whether high oxidative and ER-stress responses can induce JNK-phosphorylation during HHcy condition, I did Western blotting for p-JNK. I found that Hcy significantly induced phosphorylation of JNK in comparison with controls; however, I did not find a concomitant reduction in JNKphosphorylation via NaHS treatment as shown in Fig. 12A and 12B. To study whether JNK-phosphorylation is mediated via ER-stress mechanism(s), I also treated cells with tunicamycin (as a known ER-stress inducer). Results revealed that JNK-phosphorylation was inhibited by SP600125 (JNK-inhibitor) and induced by tunicamycin (positive control). Similar results were observed in phospho-c-Jun levels (a downstream target of JNK).
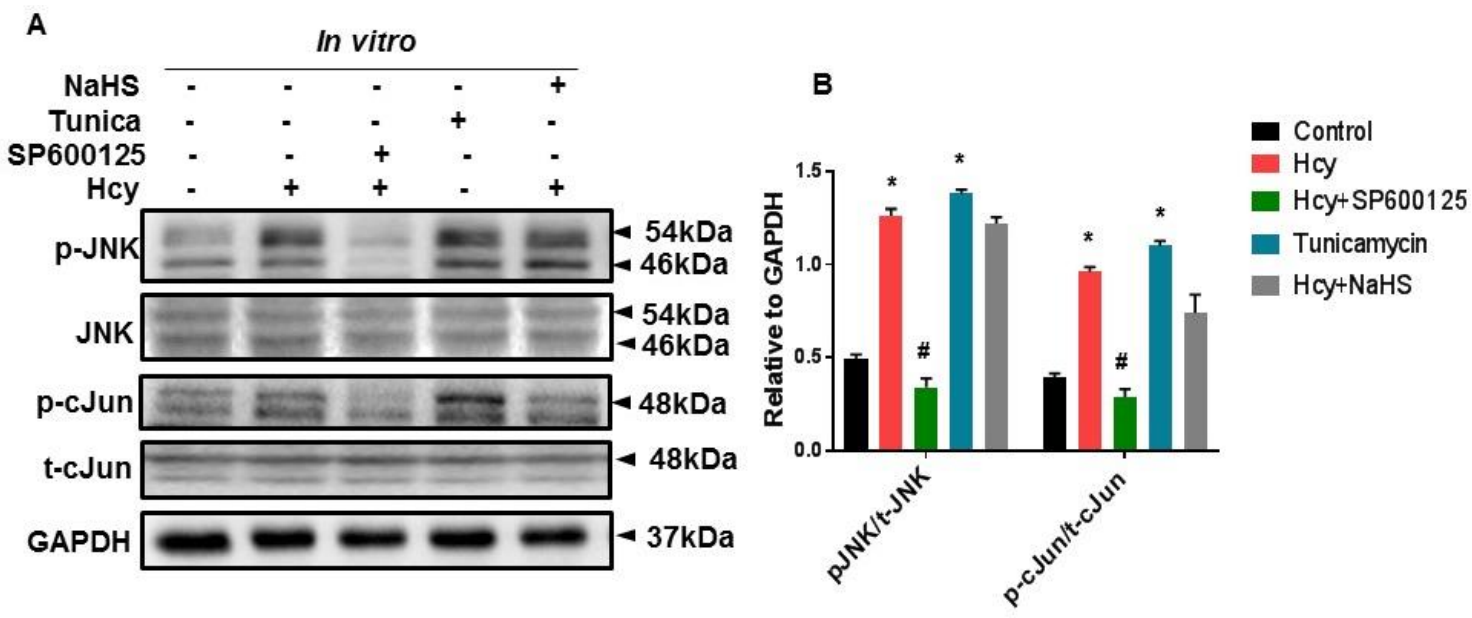


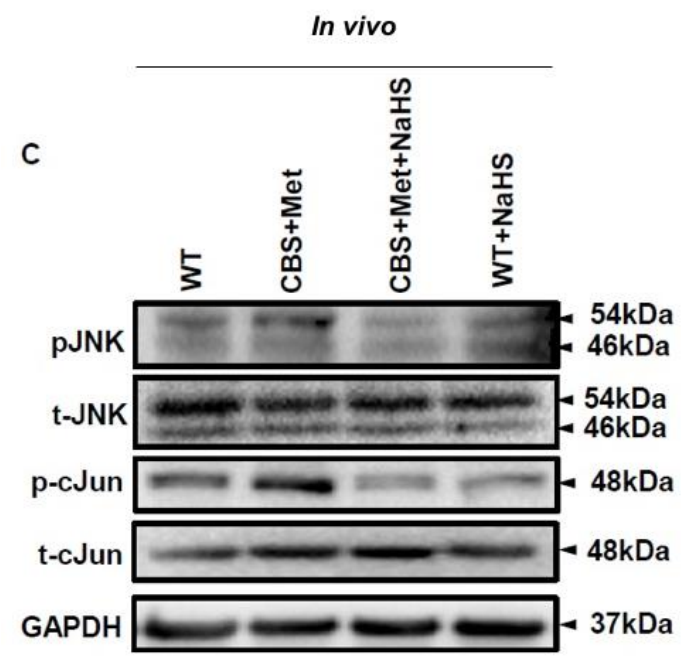

D

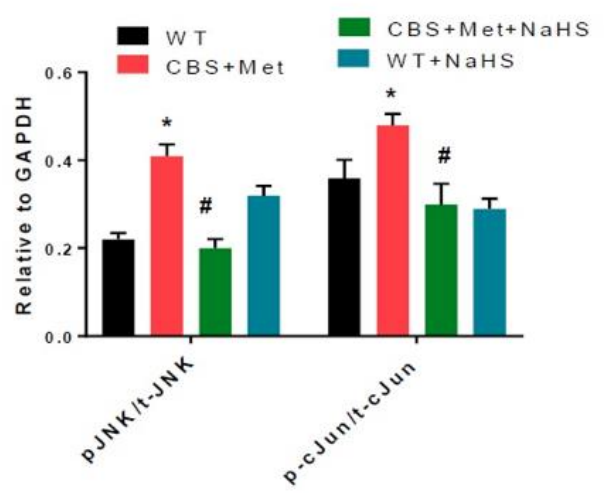

Figure 12. High Hcy induces JNK-phosphorylation in both in-vitro and in-vivo models. (A) Western blots data showing Hcy mediated JNK-phosphorylation and induced protein expression of p-cJun (S73) in C2C12 cells, where SP600125 was used as a JNK inhibitor, and Tunicamice was used as a positive control, where $n=3$ technical replicates; (B) Densitometric measurements of pJNK/t-JNK, and p-cJun/t-cJun ratios from Western blots images are shown on the right, where $n=3$ technical replicates. The interaction between multiple groups was determined by one-way or two-way ANOVA, including a Tukey's post hoc analysis when significant interaction occurred. Data are shown as mean \pm s.e.m., and ${ }^{*} p<0.05$ vs. CT and ${ }^{\#} p<0.05$ vs. Hcy; (C) Western blot data showing Hcy mediated JNK-phosphorylation and induced protein expression of $p$ cJun (S73) in CBS+Met mice compared to WT mice, where mice number $(n)=4$ in individual group; (D) Densitometric measurements of pJNK/t-JNK and p-cJun/t-cJun ratios from Western blots images are shown on the right, where mice number $(n)=4$ in individual group. The overall difference between the experimental groups was determined by one-way or two-way ANOVA, and a Tukey's post hoc analysis was run when overall statistically significant difference occurred in group means. Data are shown as mean \pm s.e.m. and ${ }^{*} p<0.05$ vs. WT and ${ }^{*} p<0.05$ vs. CBS+Met. 
Similarly, I noticed JNK-phosphorylation was induced in skeletal muscle of CBS+Met mice compared to WT mice, and interestingly this effect was mitigated via NaHS supplementation (Fig. 12C and 12D).

HHcy induced pro-inflammatory milieu in CBS+Met mice. To identify weather HHcymediated oxidative and ER-stress responses could modulate pro-inflammatory cytokines via JNK/cJun axis, I measured levels of these cytokines in plasma samples collected from experimental mice. Results showed that IL1 $\alpha$, IL2, IL4, IL6, IL12A, IFN- $\gamma$, and TNF$\alpha$, were elevated in CBS+Met in comparison with WT mice (Fig. 13). IL6 and TNF- $\alpha$ levels were found to be significantly induced in plasma of CBS+Met as compared to the WT mice.

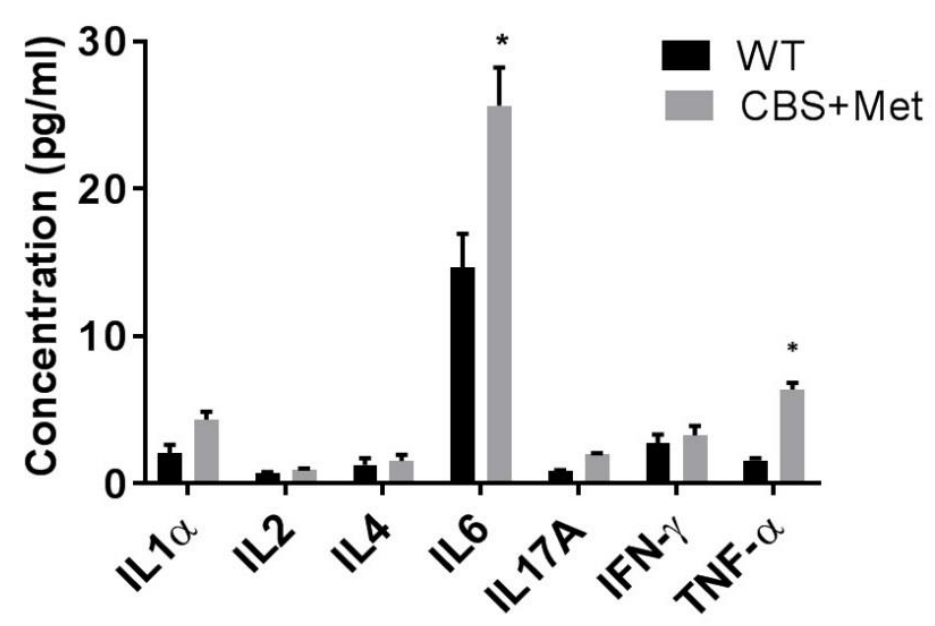

Figure 13. HHcy induced pro-inflammatory milieu in CBS+Met mice in-vivo. The levels of inflammatory cytokines such as IL1 $\alpha$, IL2, IL4, IL6, IL12A, IFN- - , and TNF- $\alpha$ were elevated in the plasma of CBS+Met mice in comparison to WT mice. The difference between these two groups were determined by unpaired t-test. Data are shown as mean \pm s.e.m. and where mice number $(n)=4$ in individual group and ${ }^{*} p<0.05$ vs. WT. 
Hcy induced cell death involves apoptosis. First, to check the cell viability after Hcy treatment, I performed MTT assay. Results showed that Hcy significantly reduced cell viability after $24 \mathrm{~h}$ and $48 \mathrm{~h}$. Although I did not find significant improvement of cell viability in Hcy treated $\mathrm{C} 2 \mathrm{C} 12$ cells after $12 \mathrm{~h}$ and $24 \mathrm{~h}$ of NaHS treatment, I noticed NaHS improved cell viability significantly after 48 h (Fig. 14A). To identify weather HHcymediated oxidative and ER-stress responses could induce apoptosis via JNK/cJun axis, I also did flow cytometry analysis after staining with Annexin $\mathrm{V}$ and propidium iodide ( $\mathrm{PI})$. Similarly, I found Hcy induced apoptosis in $\mathrm{C} 2 \mathrm{C} 12$ cells as compared to controls after 48 h, whereas NaHS treatment substantially mitigated this effect as shown in Fig. 14B and 14C.

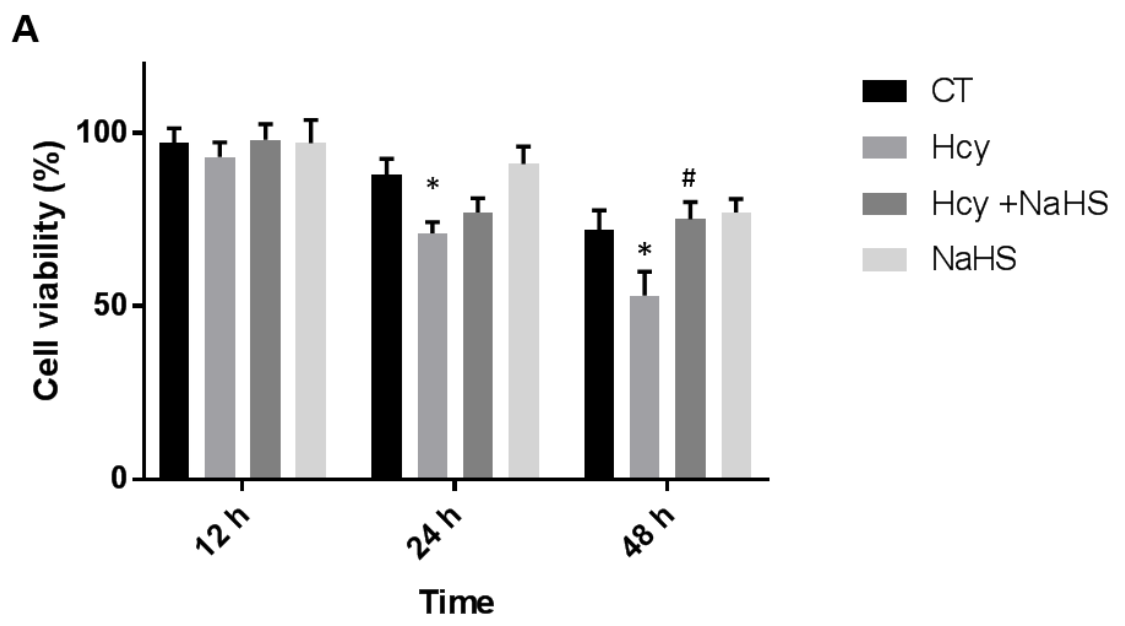


B
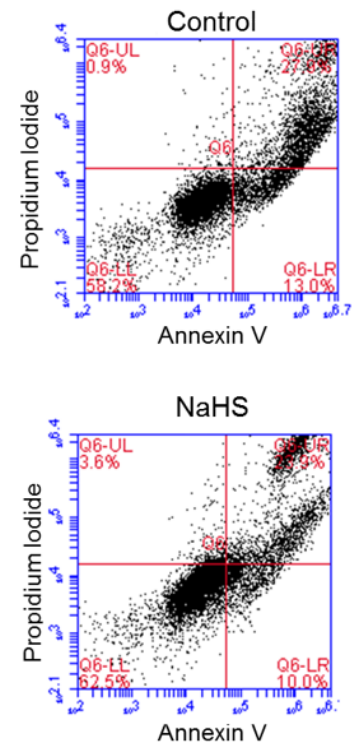
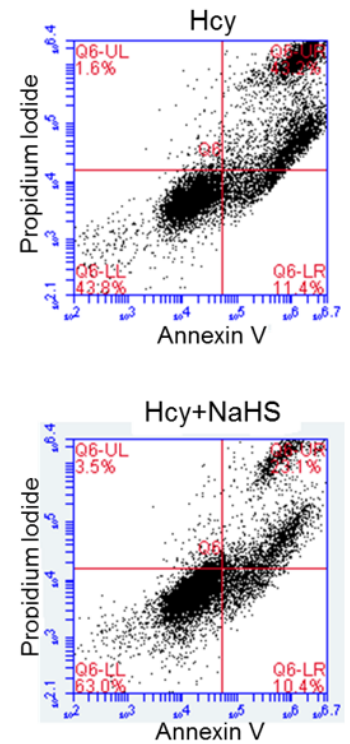

C

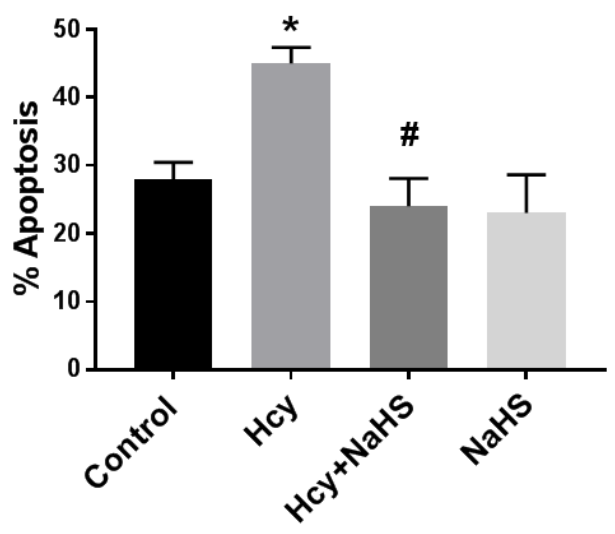

Treatments

Figure 14. NaHS protects against Hcy-induced C2C12 cells death. (A) Hcy treatment induces $\mathrm{C} 2 \mathrm{C} 12$ cell death after $24 \mathrm{~h}$ and $48 \mathrm{~h}$. Cells were treated in 4 experimental groups (CT, Hcy, Hcy+NaHS, and NaHS) for $12 \mathrm{~h}, 24 \mathrm{~h}$ and $48 \mathrm{~h}$ before being subjected to MTT assay, where $n=4$ technical replicates; (C) Flow cytometry for studying $\mathrm{C} 2 \mathrm{C} 12$ cell death by apoptosis. Representative images of PI and Annexin V expression on C2C12 cells surface, where $n=4$ technical replicates; (D) Quantification of apoptotic cells were determined by total percentage of cells which were Annexin $\mathrm{V}^{+}$and $\mathrm{PI}^{+}$, where $\mathrm{n}=4$ technical replicates. The overall difference between the experimental groups was determined by one-way or two-way ANOVA, and a Tukey's post hoc analysis was run when overall statistically significant difference occurred in group means. Data are shown as mean \pm s.e.m., and ${ }^{*} p<0.05$ vs. CT and ${ }^{*} p<0.05$ vs. Hcy.

\section{DISCUSSION}

Previously studies reported that HHcy was detrimental to skeletal muscle (20); however, molecular mechanisms underlying the detrimental effects of HHcy on muscles were not precisely studied. To my knowledge, this is the first study elaborating the 
mechanistic roles of Hcy during oxidative and ER-stress responses, which can potentiate skeletal muscle dysfunction via apoptosis, and inflammation through JNKphosphorylation.

Previous findings demonstrated the effects of HHcy on oxidative stress in cardiac microvascular endothelial cells (145), vascular smooth muscle cells (25), and liver tissue (26). The results from the present study add to the growing body of evidence that $\mathrm{HHcy}$ can, in fact, induce pathological changes in muscles via inducing ROS moieties. In this study, I also noticed lipid peroxidation and $\mathrm{H}_{2} \mathrm{O}_{2}$ levels are equally elevated, and GSH/GSSG ratio was reduced by Hcy in $\mathrm{C} 2 \mathrm{C} 12$ cells. NaHS treatment was found to improve all these conditions (Fig. 10). These results suggest that HHcy in CBS mice due to heterozygous CBS deficiency is equally detrimental to skeletal muscle compared to WT mice, which may result from higher ROS generation.

Although oxidative stress has been implicated in many diseases encompassing protein misfolding and the disruption of protein folding pathways (28-31), to my knowledge this is the first study showing that HHcy could also induce ER-stress response in skeletal muscle (Fig. 11). This finding also corroborates the previous observations showing that HHcy mediates higher ER-stress responses in endothelial cells $(150,310,311)$. A study by Malhotra et al. indicated that antioxidants reduced ERstress leading to improved protein secretion (149). Similarly, I also noticed that NaHS treatment has a beneficial effect towards mitigating ER-stress response in skeletal muscle (Fig. 11).

JNK pathway is known to control cellular response to harmful extracellular stimuli $(42,43,312)$. Whether Hcy-mediated oxidative and ER-stress responses can also induce similar effects in skeletal muscle was not studied previously. I found JNK phosphorylation was upregulated in $\mathrm{C} 2 \mathrm{C} 12$ cells after Hcy treatment, but NaHS could not reduce this effect. Whereas, my in-vivo data showed that JNK-phosphorylation was 
successfully mitigated via NaHS intervention. Since I only studied a single readout (24 hrs post-HHcy treatment) in in vitro settings, I may have missed an acute Hcy-mediated effect on the JNK-phosphorylation. In the in-vivo CBS model, I was able to observe the continuous or prolonged effect of the NaHS mediated phospho-JNK status. I noticed the apoptosis rate was induced in $\mathrm{C} 2 \mathrm{C} 12$ cells after Hcy treatment and it was mitigated by the NaHS supplementation (Fig. 14). Furthermore, reduction of apoptosis after NaHS treatment confirmed the ability of $\mathrm{H}_{2} \mathrm{~S}$ to alleviate the effects of Hcy. Moreover, this study shows that HHcy-mediated upregulation of pro-inflammatory cytokines (TNF- $\alpha$, IL-1, and IL-6) in plasma of CBS+Met mice compared to WT mice (Fig. 13). My results are also in agreement with inflammatory bowel disease conditions wherein JNK-upregulation plays a vital role (312). Oudi and colleagues reported a similar inflammatory response in acute coronary syndrome (ACS) patients, where tHcy, HsCRP, IL-6 and TNFa were significantly elevated (313). In addition to these findings, studies from other groups have reported that HHcy causes cardiovascular disease via increasing IL-1ra and IL-6 levels $(314,315)$. Furthermore, it's possible that during inflammation immune cells (CD8+ T lymphocytes) may cause myocyte degeneration (autoimmune responses) in the muscle $(14,316)$.

HHcy is known to cause hypermethylation of genes (7); however, I did not test the possibility of methylation status that may and may not be a factor in HHcy mediated apoptosis and inflammatory responses. From this study it is not still confirmed whether NaHS mediating its effects via oxidative stress mechanisms or something else and whether other antioxidants (like $\mathrm{N}$-acetylcysteine) has similar effects like NaHS. Further work is required that might throw light to explore the possible molecular mechanism(s) of the actions of $\mathrm{H}_{2} \mathrm{~S}$ and whether it could be developed as a potential therapeutic target for treating skeletal muscle dysfunction and related metabolic disorders. 


\section{CHAPTER IV}

\section{HYDROGEN SULFIDE IMPROVES POST-ISCHEMIC ANGIOGENIC DEFECT IN THE HINDLIMB OF CYSTATHIONINE- $\beta$-SYNTHASE MUTANT MICE (317)}

\section{Introduction:}

Homocysteine (Hcy) has been studied extensively in the last 30 years for its unique involvement in an increasing number of human diseases (2-4). The Hcy level is controlled by two major processes: around $50 \%$ of Hcy enters the transsulfuration pathway to produce cysteine, and the other half is re-methylated back to methionine via the folate 1-carbon cycle $(5,6)$. Normal total plasma Hcy concentration in our body ranges from 5-15 $\mu \mathrm{M}$; however, in a diseased condition, such as in hyperhomocysteinemia (HHcy), plasma total Hcy levels are increased (>15 $\mu \mathrm{M})(8)$. HHcy can be classified as moderate $(15-30 \mu \mathrm{M})$, intermediate $(30-100 \mu \mathrm{M})$ and severe $(>100 \mu \mathrm{M})(8)$. Notably, there are 4 ways people can develop HHcy: (1) consumption of a methionine-rich protein diet; (2) vitamin $\mathrm{B}_{12} /$ folate deficiency; (3) presence of heterozygous/homozygous status for cystathionine- $\beta$ synthase $\left(\mathrm{CBS}^{+/} / \mathrm{CBS}^{-/}\right)$; and (4) obstruction of renal clearance (12). Apart from these factors, genetic variants in Hcy metabolism enzymes such as $677 \mathrm{C}>\mathrm{T}$ and $1298 \mathrm{~A}>\mathrm{C}$ in the MTHFR gene can also lead to HHcy $(14,76-78)$. There are also several other drivers like age, sex, physical activity, alcohol intake, certain medications and different disease conditions 
(such as type 2-diabetes) that can also modulate the functioning methionine cycle, leading to an increased total Hcy concentration in the blood (79). HHcy has been associated with severe skeletal muscle dysfunction (7,13-20), but the precise mechanism(s) is still unknown. Previous studies found that HHcy causes endothelial cell (EC) injury (209), inhibition of EC proliferation (318), reduction of bioavailability of vasoregulatory mediators (such as NO and endothelin) (319), and induction of oxidative/ER-stress $(24,33,320)$. How HHcy reduces neovascularization in the skeletal muscle is not precisely known.

The growth of new blood vessels from the preexisting vascular network is known as angiogenesis. When oxygen supply is low in a tissue or organ, it activates HIF1a, which sends a signal to the nearest blood vessel, activating eNOS and producing NO needed for vasodilation. VEGF increases permeability and separates pericytes leading to the degradation of the basement membrane, thereby activating metalloproteases such as MMP-2 and 9 (321). These changes lead to EC proliferation and a concomitant migration in order to form new blood vessels (322). In recent years, it has been shown that PPAR-y might be involved in angiogenesis via growth factors and cytokines that in turn stimulate migration, proliferation, and survival of these ECs (323).

PPAR-y belongs to the nuclear hormone receptor superfamily; when specific ligands bind to the ligand-binding domain of PPAR- $\mathrm{y}$, a conformational change releases the bound corepressors $(324,325)$. This allows co-activators like PGC-1 $\alpha$ and other coactivators to be recruited to the PPAR-y responsive gene promoters thereby promoting the PPAR- $y-$ mediated transcription $(81,326,327)$. HHcy reduces PPAR- $\gamma$ expression in ECs (148). Studies have shown that PPAR-y could regulate angiogenesis via upregulating VEGF (323) and that can further activate eNOS (328). Although, previous works showed that HHcy impaired neoangiogenic growth in muscle via reduction of HIF1 $\alpha$ and VEGF levels, whether PPAR-y plays any role in this process had 
not been studied (46).

$\mathrm{H}_{2} \mathrm{~S}$ is increasingly being recognized as an important signaling molecule in cardiovascular and nervous systems via its ability to neutralize a variety of reactive oxygen species (ROS) (51-53), and via increased cellular GSH levels through activation of gamma-glutamylcysteine synthetase, and reduction of the disulfide bonds (5461,329). CBS and CSE are the main $\mathrm{H}_{2} \mathrm{~S}$ generating enzymes, producing $\mathrm{H}_{2} \mathrm{~S}$ from $\mathrm{Hcy}$ in the transsulfuration pathway (330). Patients with CBS deficiency tend to produce a lesser amount of $\mathrm{H}_{2} \mathrm{~S}(63,236)$, suggesting that these patients are likely more prone to oxidative stress-mediated damages due to excessive production of Hcy (54). A study revealed that endogenous $\mathrm{H}_{2} \mathrm{~S}$ could induce mRNA and protein expression of PPAR- $\gamma$, indicating that exogenous $\mathrm{H}_{2} \mathrm{~S}$ supplementation could be employed as a beneficial strategy to improve angiogenesis defect in HHcy patients (331). Hence the purpose of my study was to answer the following questions: (i) Does HHcy inhibit angiogenesis via downregulation of angiogenic signals like HIF1 $\alpha$ and VEGF in 21 days of post-FAL hindlimb of $\mathrm{CBS}^{+/-}$mice? (ii) Does HHcy inhibit PPAR-y expression which can further downregulate VEGF/eNOS signaling in the post-FAL hindlimb of $\mathrm{CBS}^{+/-}$mice? And finally, (iii) Does GYY4137 (an $\mathrm{H}_{2} \mathrm{~S}$ donor) treatment improve angiogenesis via PPARY/VEGF axis after 21 days of FAL in the hindlimb of experimental $\mathrm{CBS}^{+/-}$mice?

CBS is one of the key enzymes in the transsulfuration pathway; heterozygous CBS deficiency $\left(\mathrm{CBS}^{+/-}\right)$has proved to be a useful model for analyzing the effects of mild to a severe endogenous elevation in the levels of Hcy (64-71). Hence, in this study, I used $\mathrm{CBS}^{+/-}$mouse model to identify the effect(s) of HHcy on angiogenesis in the skeletal muscle and evaluate whether exogenous administration of GYY4137 could improve this effect(s). Results indicate that $\mathrm{H}_{2} \mathrm{~S}$ could be developed as a potential therapeutic agent to treat the neoangiogenic defects in skeletal muscle wherein HHcy is linked with a barrage of metabolic dysfunctions. 


\section{MATERIALS AND METHODS}

Animal maintenance, genotyping and diet protocol. Male WT (C57BL/6J) and $\mathrm{CBS}^{+/-}$(B6.129P2-Cbstm1Unc/J 002853) mice were purchased from the Jackson Laboratory (Bar Harbor, ME). All animals were 8-10 weeks-old and maintained in 12:12 $\mathrm{h}$ light-dark cycle with regular mouse chow diet in the animal facility of the University of Louisville. All animal protocols and care were carried out according to the guidelines of National Institute of Health (NIH Pub. No. 86-23, revised 1985) and were approved by the Institutional Animal Care and Use Committee (IACUC) of the University of Louisville, KY, USA.

After purchasing, mice were cross-bred, yielding around $10 \% \mathrm{CBS}^{-1-}, 60 \%$ $\mathrm{CBS}^{+/-}$, and $25 \% \mathrm{CBS}^{+/+}$. For genotyping, tail samples were collected, and DNA was isolated using DNeasy Blood \& Tissue Kits (Qiagen, Germantown, MD, USA). Genotypic analysis was performed using PCR by targeted disruption of the CBS gene (representative images from each group of post-FAL mice are shown in Fig. 15A and genotyping in Fig. 15B). $\mathrm{CBS}^{+/-}$heterozygote mice produced two bands (450 and 308 bp), while wild-type $\left(\mathrm{CBS}^{+/+}\right)$mice represented only one band (308bp). Animals were divided into 4 experimental groups: (1) Wild-type C57BJ/L6 mice (WT), (2) $\mathrm{CBS}^{+/-}$ heterozygous mice (CBS) (3) GYY4137-supplemented CBS ${ }^{+/}$(CBS+GYY), (4) GYY4137-supplemented wild-type mice (WT+GYY). $0.25 \mathrm{mg} \mathrm{GYY4137/kg} \mathrm{body} \mathrm{weight}$ of mouse was applied by intraperitoneal injection every day for a total 21 days after femoral artery ligation (FAL) surgery, while the WT mice were given $0.9 \%$ normal saline (vehicle control) (72).

Femoral artery ligation (FAL). To create a hypoxic condition femoral artery ligation (unilateral) was performed under intraperitoneal pentobarbital sodium $(50 \mathrm{mg} / \mathrm{kg}$ ) anesthesia $(50,51)$. Briefly, after separation of the femoral artery from the vein and 
nerve, it was ligated using 6-0 silk suture at proximal and distal places (keeping same distance in all animals). I used separate mice groups [WT, CBS, CBS+GYY, and WT+GYY] as sham control where I performed the full process of surgery except the ligation. The skin was sutured using 6-0 silk thread. After the skin closure, betadine was applied. After recovery, laser Doppler blood perfusion was carried out to confirm the induction of ischemia.

Laser-Doppler tissue perfusion imaging and flowmetry. MoorLDI (Moor Instruments) was used to measure tissue perfusion intensity and blood flow rates (332). In brief, after anesthesia, a part of skin in hind limb was opened and blood flow measurement was taken for 2 mins keeping same laser pointer in all experimental mice.

Barium angiograms. To determine angiogenesis, barium sulfate angiography was performed in mice (333). In brief, after pentobarbital anesthesia mice were infused with barium sulfate $(0.1 \mathrm{~g} / \mathrm{mL})$ in $50 \mathrm{mM}$ Tris-buffer $(\mathrm{pH} 5.0)$ at a constant flow $(\sim 1 \mathrm{ml} / \mathrm{min})$ and pressure with a syringe pump through the common carotid artery. Heparin $(20 \mathrm{U} / \mathrm{ml})$ was used along with barium sulfate to visualize the nascent angiogenesis. Angiograms were captured using the Carestream whole animal X-ray imaging system (Carestream Molecular Imaging, Woodbridge, CT) (334) and the vessel density was quantified using VesSeg tool (Institute for Signal Processing, University of Luebeck, Lübeck, Germany).

Reagents and antibodies. All reagents and chemicals were ordered from Sigma-Aldrich or available highest grade.

The antibodies for HIF1a (ab51608), VEGF (ab51745) and eNOS (ab66127) were ordered from Abcam (Cambridge, MA, USA). Whereas PPAR-ץ (sc-7273), p-eNOS $\operatorname{Ser}^{1177}$ (sc-12972), rabbit anti-mouse (sc-358914), mouse anti-rabbit (sc-2357), and mouse anti-goat (sc-2354) were from Santa Cruz Biotechnology (Dallas, TX, USA). The antibody for GAPDH (MAB374) was from EMD Millipore (Burlington, MA, USA), and 
used for Western blots analyses as per the manufacturers' protocols.

Western blotting. Protein expressions were assessed by Western blots (72). Briefly, at the time of sacrifice, gastrocnemius muscle from the ischemic leg of each mouse was quickly removed, snap-frozen, and stored at $-80^{\circ} \mathrm{C}$ until further use. Protein from samples was extracted by homogenizing in the ice-cold RIPA buffer (Boston BioProducts, Worcester, MA, USA) containing $1 \mathrm{mM}$ Phenylmethylsulfonyl fluoride (Sigma, Saint Louis, MO, USA), 1\% protease inhibitors cocktail (Sigma, Saint Louis, MO, USA) and then sonicated employing the Sonifier 450 (Branson Ultrasonics, Danbury, CT, USA) using $3 \times 5 \mathrm{sec}$ at setting " 3 ". The homogenates were centrifuged 17,400 × $g$ for $20 \mathrm{~min}$ at $4{ }^{\circ} \mathrm{C}$, and the supernatants were quickly stored at $-80^{\circ} \mathrm{C}$ until further use. The protein contents were estimated by the Bradford assay. Equal amounts of proteins $(50 \mu \mathrm{g})$ were resolved on SDS-PAGE $(8 \%, 10 \%, 12 \%)$ and then transferred to polyvinylidene difluoride (PVDF) membranes. The respective blots were incubated with primary and secondary antibodies before visualizing them using the ECL Luminata Forte (Millipore, Temecula, CA, USA) in a Bio-Rad ChemiDoc system. The intensities of the bands were normalized to the housekeeping GAPDH for all the proteins examined. The quantification was performed using Image Lab ${ }^{\text {TM }}$ Software (Bio-Rad, Hercules, CA, USA).

Reverse transcription and real-time quantitative PCR. Total RNA was extracted from muscle samples using a Trizol method as mentioned in chapter III (306). Then RNA quality was determined by NanoDrop ND-1000, and RNA with high purity (260/280 2.00 and 260/230 2.00) was used for q-PCR analysis. Reverse transcription was performed according to the manufacturer's protocol using high-capacity cDNA RT kit from Applied Biosystems (Foster City, CA, USA) for the primer sequences listed in Table 3. For RTQPCR, SYBR green-based kit was used to measure the relative expression of each mRNA specific primers. Briefly, 3 steps cycling protocol was performed using $20 \mathrm{ng}$ of 
cDNA template in a $20 \mu \mathrm{L}$ reaction volume under the following conditions: denaturation at $95^{\circ} \mathrm{C}$ for $15 \mathrm{~min}$ followed by 40 cycles of $94^{\circ} \mathrm{C}$ for $15 \mathrm{~s}, 55^{\circ} \mathrm{C}$ for $30 \mathrm{~s}$, and $70{ }^{\circ} \mathrm{C}$ for $34 \mathrm{~s}$ in which fluorescence was acquired and detected by Roche LightCycler 96 RealTime PCR System (Roche Diagnostics, Indianapolis, IN, USA). Following RT-qPCR, analysis of melt curve was performed to validate the specific generation of the expected PCR product. GAPDH was used as an endogenous control (Quanta Biosciences, Beverly, MA, USA).

\begin{tabular}{|c|c|c|}
\hline Genes & Forward Primers & Reverse Primers \\
\hline HIF1 $\alpha$ & 5'- TCAAGTCAGCAACGTGGAAG-3' & 5'- TATCGAGGCTGTGTCGACTG-3' \\
\hline VEGF & 5'- CAGGCTGCTGTAACGATGAA-3' & 5'- CAATTTGGCTCCTCCTACCA -3' \\
\hline PPAR-Y & 5'- TTTTCAAGGGTGCCAGTTTC-3' & 5'- AATCCTTGGCCCTCTGAGAT-3' \\
\hline NOS3 & 5'- GACCCTCACCGCTACAACAT-3' & 5'- TCTGGCCTTCTGCTCATTTT -3' \\
\hline
\end{tabular}

Table 3. List of primers used for RT-qPCR experiments for chapter V.

Total plasma Homocysteine, hydrogen sulfide, and nitrite measurement. Blood samples were collected in tubes containing a $1 / 10$ volume of $3.8 \%$ sodium citrate from each mouse by cardiac puncture after euthanasia. Then plasma was isolated by centrifugation at $2500 \times \mathrm{g}$ for $15 \mathrm{mins}$ at $4^{\circ} \mathrm{C}$. Total plasma Hcy concentrations were measured in samples using the homocysteine assay kit (Crystal Chem, IL, USA) as per manufacturer's instructions.

Plasma $\mathrm{H}_{2} \mathrm{~S}$ was measured as a previously described method (208). In brief, samples were centrifuged at a speed of $\times 11,000 \mathrm{~g}$ for 10 minutes. $100 \mu$ laliquots from the plasma were mixed separately with PBS $(350 \mu \mathrm{L})$ and $\mathrm{Zn}\left(\mathrm{O}_{2} \mathrm{CCH}_{3}\right) 2(1 \% \mathrm{~W} / \mathrm{V}, 250 \mu \mathrm{L})$ 
in a micro-centrifuge tube and sealed immediately. Then, N, N-dimethyl-pphenylenediamine sulfate $(20 \mathrm{mM}, 133 \mu \mathrm{L})$ in $7.2 \mathrm{M} \mathrm{HCl}$, and $\left.\mathrm{FeCl}_{3} 30 \mathrm{mM}, 133 \mu \mathrm{L}\right)$ in 1.2 $\mathrm{M} \mathrm{HCl}$ was added to the mixture, sealed and incubated at $37^{\circ} \mathrm{C}$ for $45 \mathrm{~min}$. After that, Trichloroacetic acid solution $(10 \% \mathrm{w} / \mathrm{v}, 250 \mu \mathrm{L})$ was added to the mixture to terminate the reaction. After centrifugation ( $\times 2,700 \mathrm{~g}$ for 5 mins), $200 \mu \mathrm{L}$ supernatant was transferred to a 96-well plate and the absorbance was measured at $670 \mathrm{~nm}$ in a spectrophotometer. $\mathrm{H}_{2} \mathrm{~S}$ levels were calculated against a calibration curve of known concentrations of NaHS (0.01- $100 \mu \mathrm{mol} / \mathrm{L})$.

Both nitrite and nitrate from the plasma was measured by a Griess reagent as (301). Briefly, equal volume of Griess reagent [0.1\% N-(1-naphthyl) ethylenediamine dihydrochloride, $1 \%$ sulfanilamide, and $2.5 \%$ phosphoric acid] was added to $100 \mu \mathrm{L}$ of samples and controls [sodium nitrite $\left(\mathrm{NaNo}_{2}\right)$ ] (0.01-100ug). Then samples were incubated at $37^{\circ} \mathrm{C}$ for $30 \mathrm{~min}$ and absorbance was recorded at $542 \mathrm{~nm}$ in a Spectra Max M2 plate reader.

Statistics. All values are expressed as mean \pm s.e.m. The interaction between groups was determined by one-way or two-way ANOVA, including a Tukey's post hoc analysis when significant interactions were observed. The threshold for significance was set at $p<0.05$, the total number of mice $(n)=4-5$ were subjected to experimentation from each group. For statistical analyses, GraphPad Prism (Ver 7, GraphPad Software) was used.

\section{RESULTS}

The phenotypic feature and genotype of WT and $\mathrm{CBS}^{+/-}$mice are depicted in Fig. $15 \mathrm{~A}$ and $15 \mathrm{~B}$ respectively. In this study, I noticed that CBS mice had significantly lower body weights in comparison to WT mice; however, I did not see any difference in body weights between CBS vs. WT after GYY4137 treatment for 21 days (Fig. 15C). I 
observed CBS mice had significantly higher levels of plasma tHcy compared to WT mice, GYY4137 supplemented CBS mice also had similarly higher plasma tHcy levels as that of the CBS mice (Fig. 15D). After 21 days of GYY4137 treatment, I examined the plasma $\mathrm{H}_{2} \mathrm{~S}$ concentrations in the experimental mice. Results showed that plasma $\mathrm{H}_{2} \mathrm{~S}$ levels were significantly lower in the CBS mice compared to that of the WT mice as expected (Fig. 15E). However, after administration of GYY4137 for 21 days plasma $\mathrm{H}_{2} \mathrm{~S}$ levels were significantly elevated in both the CBS and WT mice (Fig. 15E).

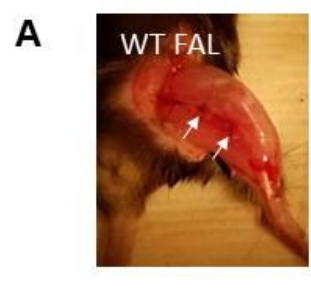

B

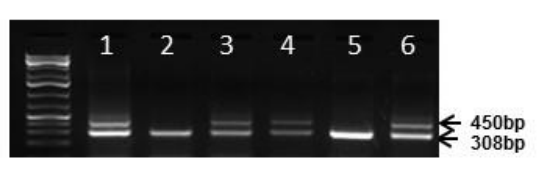
$\begin{array}{ll}\text { Wild type: } 308 \text { bp } & \text { CBS }^{+/-:}: \text {lane 1, 3,4 and } 6 \\ \text { Heterozygous: } 308 \text { and } 450 & \text { WT }\left(\mathrm{CBS}^{+++}\right) \text {: lane } 2 \text { and } 5\end{array}$

D

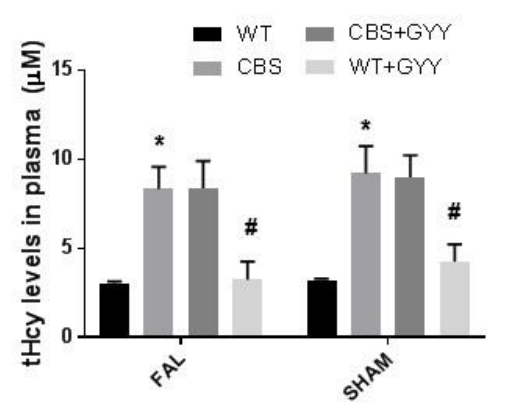

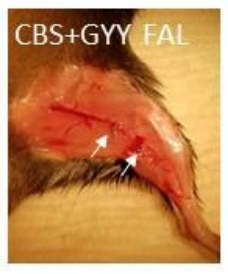
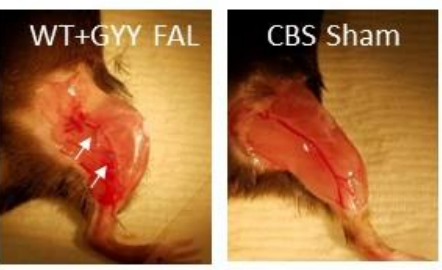

C

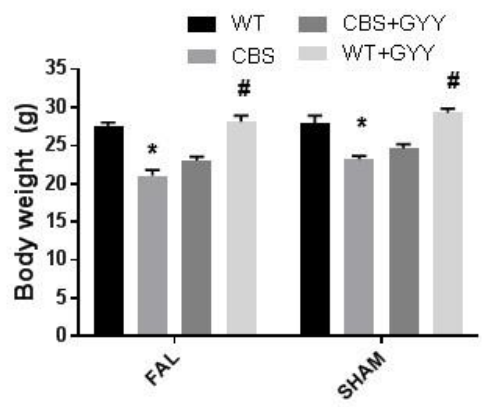

E

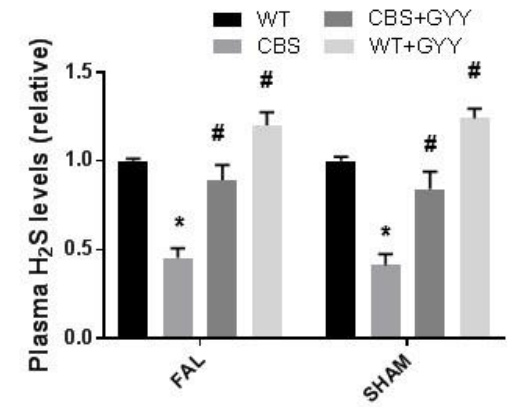

Figure 15. Phenotypic and genotypic characteristics of experimental mice groups. (A) Hindlimb images after 21 days of GYY4137 treatments; (B) Genotyping of experimental mice; (C) Body weight measurements of experimental mice, where mice number $(n)=4$ in individual group; (D) tHcy measurements from the plasma of experimental mice, where mice number $(n)=4$ in individual group; $(E) H_{2} S$ measurements from the plasma of 
experimental mice, where mice number $(n)=4$ in individual group. The overall difference between the experimental groups was determined by one-way or two-way ANOVA, and a Tukey's post hoc analysis was run when overall statistically significant difference occurred in group means. Data are shown as mean \pm s.e.m. and statistical difference ${ }^{*} \mathrm{p}<0.05$ vs. WT and ${ }^{\#} \mathrm{p}<0.05$ vs. CBS. (tHcy=total homocysteine, FAL=femoral artery ligation).

As a marker of a hypoxia induction in post-FAL hindlimb, I measured the HIF1a levels by Western blotting. I noticed that HIF1 $\alpha$ levels were downregulated in post-FAL CBS mice in comparison to WT mice, GYY4137 treatment was found to mitigate this effect (Fig. 16A). To confirm mRNA expression of HIF1a, I did qPCR analysis, and it did not show any significant decrease in mRNA levels of HIF1 $\alpha$ in post-FAL CBS mice compared to post-FAL WT ( $\mathrm{p}=0.4824)$, GYY4137 treatment could not improve the mRNA levels in post-FAL CBS mice $(p=0.9781)$ (Fig. 16B). I found that the protein expressions of VEGF and PPAR- $\gamma$ were reduced in post-FAL CBS mice as compared to post-FAL WT mice, whereas this effect was improved after GYY4137 administration (Fig. 16A). Additionally, in the qPCR analysis, I found mRNA expression of VEGF was significantly reduced in the post-FAL CBS mice compared to post-FAL WT mice $(p=0.0365)$; however, this effect was not improved upon GYY4137 administration $(p=0.2139)$. I did not notice any significant change in the mRNA expression of PPAR- $\gamma$ among the 4 experimental groups (Fig. 16B). I did not find any difference in proteins and mRNA levels for HIF1 $\alpha$ and VEGF among individual groups of sham mice as shown in Fig 16C-D. However, I did notice that the expression of PPAR-y was reduced in sham CBS mice compared to sham WT mice and that GYY4137 supplementation could not mitigate this effect. The observed reduction of PPAR-y mRNA level in sham CBS mice, 
compared to sham WT mice, was not statistically significant $(p=0.7423)$. Similarly, I did not observe any significant improvement in mRNA expression for PPAR-y in sham CBS mice after GYY4237 treatment ( $p=0.3549)$ (Fig. 16D).

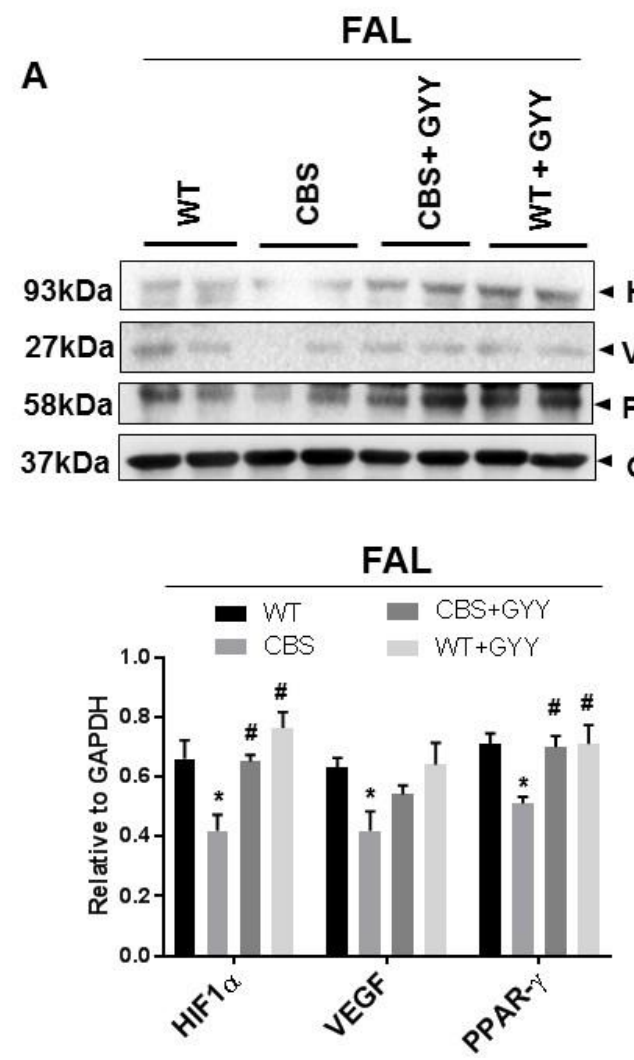

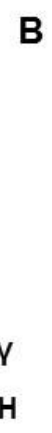

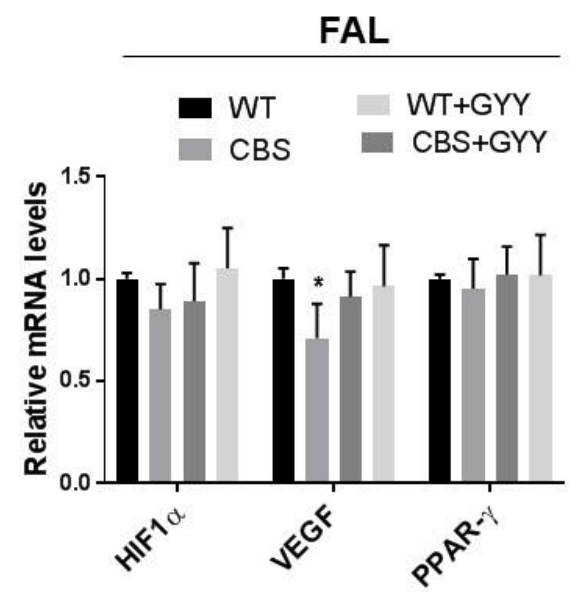

Figure 16A-B. Effect of GYY4137 on the improvement of angiogenic markers in skeletal muscle of post-FAL mice. (A) Western blot analysis is showing protein expressions: HIF1- $\alpha$, VEGF, and PPAR- $\gamma$ in the top panel and densitometric analysis of Western blot images are shown in the bottom panel, where mice number $(n)=4$ in individual group; (B) mRNA expression for HIF1- $\alpha$, VEGF, and PPAR-y (log transformed) in skeletal muscle of post-FAL mice, where mice number $(n)=4$ in individual group. The overall difference between the experimental groups was determined by one-way or two-way ANOVA, and a Tukey's post hoc analysis was run when overall statistically significant difference occurred in group means. Data are shown as mean \pm s.e.m. and statistical difference 
${ }^{*} \mathrm{p}<0.05$ vs. WT and ${ }^{\#} p<0.05$ vs. CBS ( $F A L=$ femoral artery ligation).

C

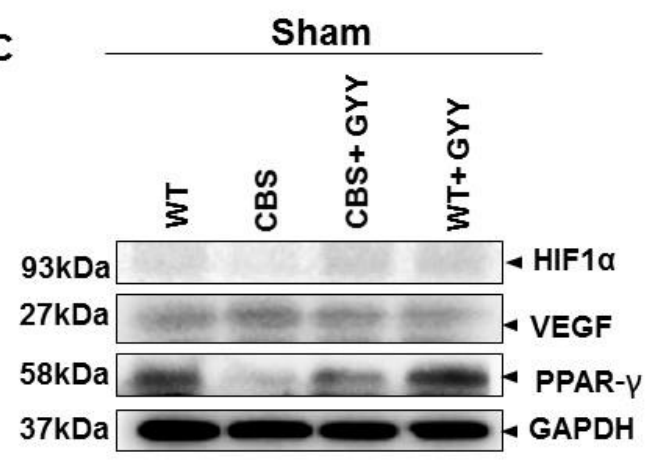

Sham

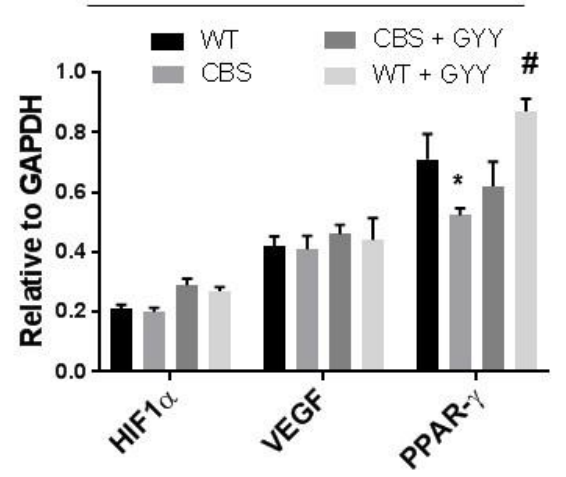

D

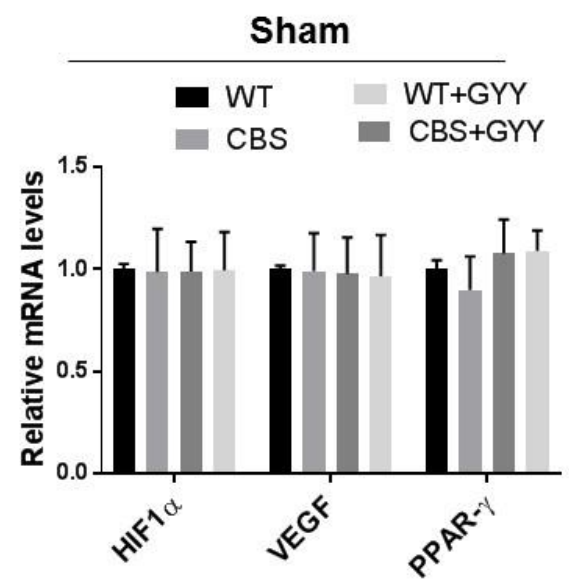

Figure 16C-D. Effect of GYY4137 on angiogenic markers in skeletal muscle in sham mice. (C) Western blot analysis is showing protein expressions: HIF1-a, VEGF, and PPAR-y in the top panel and densitometric analysis of Western blot images are shown in the bottom panel, where mice number $(n)=4$ in individual group; (D) mRNA expression for HIF1- $\alpha$, VEGF, and PPAR-y (log transformed), where mice number $(n)=4$ in individual group. The overall difference between the experimental groups was determined by oneway or two-way ANOVA, and a Tukey's post hoc analysis was run when overall statistically significant difference occurred in group means. Data are shown as mean \pm s.e.m., statistical difference ${ }^{*} p<0.05$ vs. WT and ${ }^{*} p<0.05$ vs. CBS.

Finally, I measured the vessel density employing barium sulfate angiography 
after 21 days of FAL surgery. It was noticed that total collateral vessel numbers were significantly less in the post-FAL CBS mice in comparison to post-FAL WT mice, this effect was further significantly improved upon GYY4137 treatment (Fig. 17A-B). Besides, blood flow in the hindlimb after 21 days of FAL was reduced considerably in CBS mice compared to WT mice, and it was improved by GYY4137 treatment as seen in Fig. 17CD. Although I did not notice any difference in the mRNA expression levels of NOS3, I observed that the p-eNOS levels were reduced in the post-FAL CBS mice as compared to post-FAL WT mice, interestingly, this effect was improved via GYY4137 treatment (Fig. 17E). The changes in the plasma nitrite levels in experimental mice were also monitored as the marker of nitric oxide (NO) production. Although not statistically significant, my findings revealed a reduction of plasma nitrite levels in post-FAL CBS mice as compared to post-FAL WT mice $(p=0.1050)$, and similarly, I noticed a trend of improvement of this effect with the GYY4137 administration ( $p=0.2923)$ (Fig. 17F). 

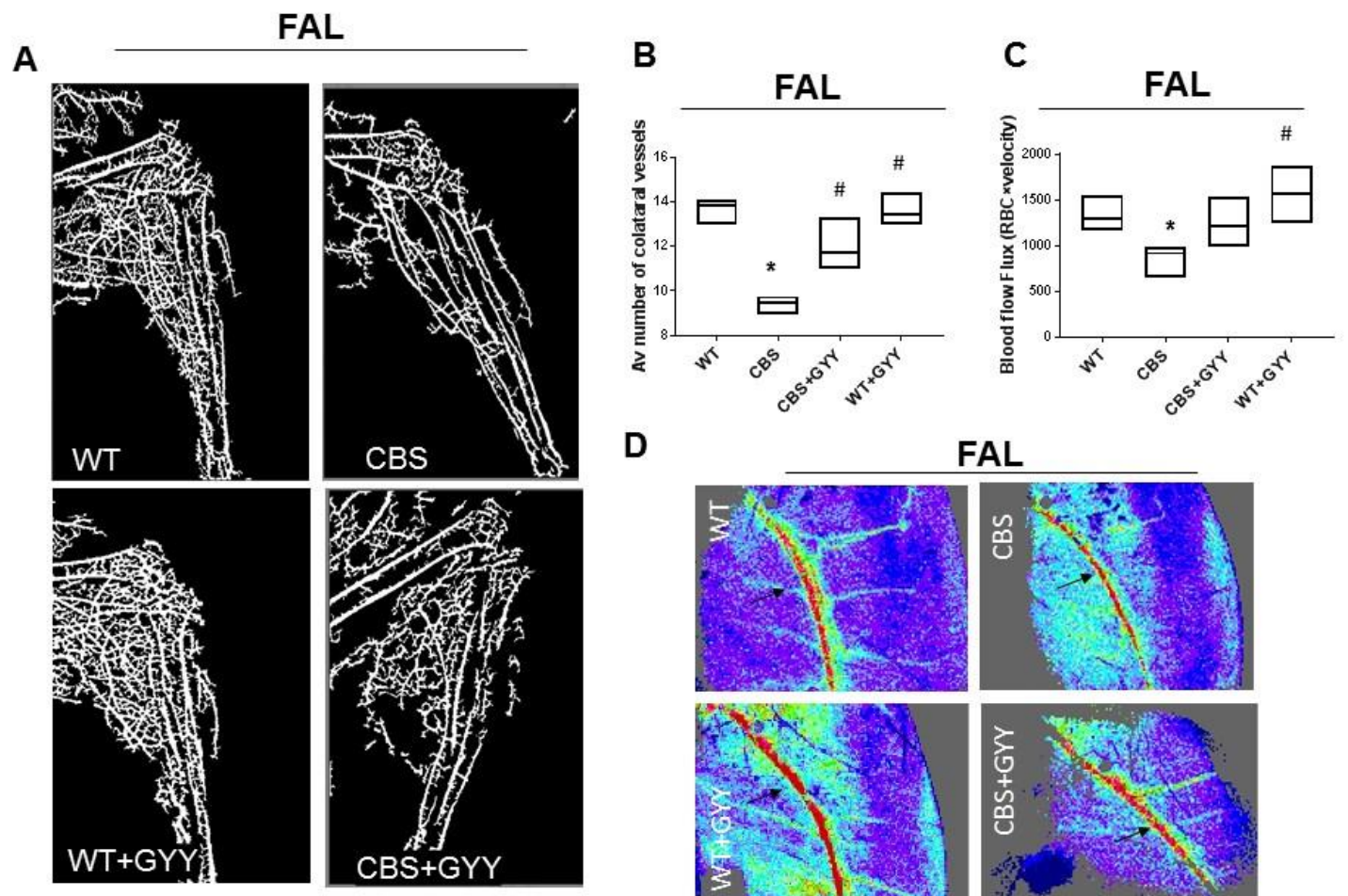

D

FAL
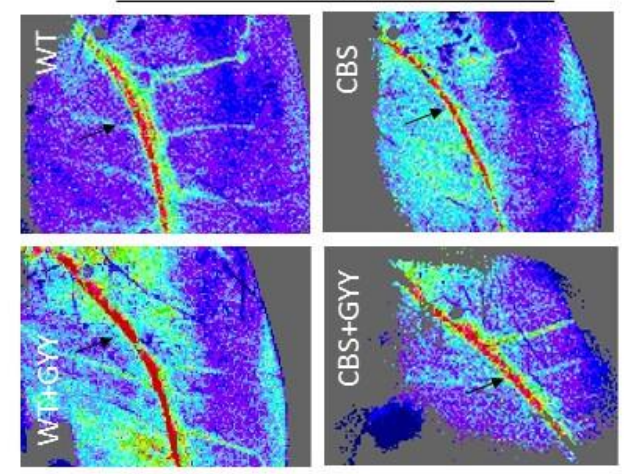

Figure 17A-D. GYY4137 supplementation improves angiogenesis in the hindlimb muscle after 21 days of $\mathrm{FAL}$ in $\mathrm{CBS}^{+/-}$mice. (A) Barium angiogram images showing vascular density in the hindlimb of skeletal muscle; (B) Quantitation of collateral vessel numbers in hindlimb post FAL mice, where mice number $(n)=4$ in individual group; $(C)$ Blood flow rate measurements in hindlimb post FAL mice, where mice number $(n)=4$ in individual group; (D) Laser-Doppler perfusion imaging showing the intensity of limb perfusion 21 days post FAL mice. The overall difference between the experimental groups was determined by one-way or two-way ANOVA, and a Tukey's post hoc analysis was run when overall statistically significant difference occurred in group means. Data are shown as mean \pm s.e.m. and statistical difference ${ }^{*} p<0.05$ vs. WT and ${ }^{\#} p<0.05$ vs. $C B S$. ( $F A L=$ femoral artery ligation). 

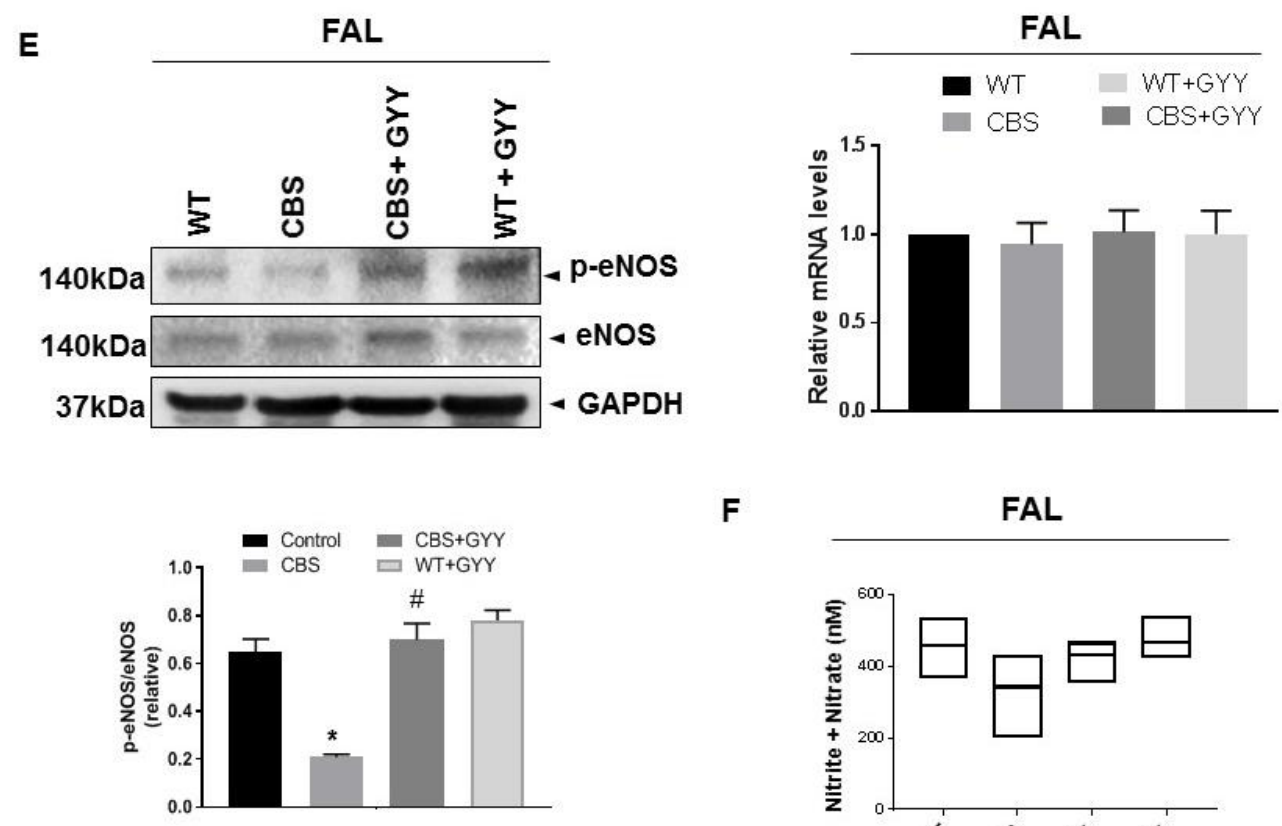

$\mathbf{F}$

FAL

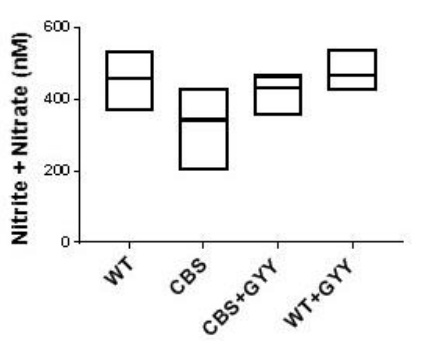

Figure 17E-F. Effect of GYY4137 supplementation on eNOS-phosphorylation and plasma nitrite levels in experimental mice. (E) Western blot analysis of protein expressions: $p$-eNOS and eNOS in the top panel and densitometric analysis of $p$ eNOS/eNOS ratio from Western blot images are shown in the right panel, and mRNA expression of NOS3 is shown in bottom panel, where mice number $(n)=4$ in individual group; (F) Nitrite+Nitate levels in plasma of post-FAL mice measured by Griess assay, where mice number $(n)=4$ in individual group; The overall difference between the experimental groups was determined by one-way or two-way ANOVA, and a Tukey's post hoc analysis was run when overall statistically significant difference occurred in group means. Data are shown as mean \pm s.e.m. and statistical difference ${ }^{*} \mathrm{p}<0.05$ vs. WT and ${ }^{\#} \mathrm{p}<0.05$ vs. CBS. (FAL=femoral artery ligation)

\section{DISCUSSION}


Earlier studies also showed that HHcy has a profound inhibitory effect on EC proliferation and their migration (269,270,335-337). In the present study, I examined whether HHcy condition could impair angiogenesis in-vivo during a chronic ischemic insult. Although there are many in-vivo models available to study angiogenesis, either by passing a flexible wire or by applying a laser or an electrical current, none of these are relevant to the clinical setting (338-340). In this study, I used the femoral artery ligation (FAL) model by employing a genetically engineered mouse model $\left(\mathrm{CBS}^{+/}\right)$mimicking HHcy conditions as seen in HHcy patients with CBS mutation to study the post-ischemic angiogenesis phenomenon during $\mathrm{HHcy}$. My results showed that $\mathrm{HHcy}$ is associated with defective angiogenesis, and exogenous $\mathrm{H}_{2} \mathrm{~S}$ supplementation has a protective role in mitigating this effect.

Angiogenesis is a natural process during chronic regional ischemia, which requires EC proliferation, migration, differentiation, and survival to form new blood vessels in order to compensate for the hypoxic environment (341). VEGF is a prototypical angiogenic cytokine that plays a vital role in this process and has been widely studied (342-344). A previous report involving hindlimb ischemia in $\mathrm{CBS}^{+/-}$mice showed no difference in VEGF levels 7 days of post-ischemia (345). However, they did notice a significant reduction in capillary density in the CBS mice compared to WT mice after 21 days of ischemia (345). Interestingly, my results demonstrate a significant decrease in PPAR- $\gamma$ and VEGF expression after 21 days of FAL, suggesting that lower expressions of post-ischemic PPAR- $\gamma$ may be responsible for delayed induction of VEGF in CBS mice compared to that of WT mice (323). I noticed that the exogenous administration of GYY4137 could improve blood flow and collateral vessel density in skeletal muscle of $\mathrm{CBS}^{+/-}$mice (Fig. 17C).

$\mathrm{H}_{2} \mathrm{~S}$ has been studied extensively for its positive effects in the cardiovascular 
system, demonstrating profound vasodilatation, vascular protection, homeostatic regulation of blood pressure and many others $(54,57-59,61)$. A previous study using chicken chorioallantoic membrane model revealed that $\mathrm{H}_{2} \mathrm{~S}$ increased the length and complexity of the vascular network (270). Likewise, in this study, I noticed that exogenous supplementation of GYY4137 could improve collateral vessel density after 21 days of FAL in the $\mathrm{CBS}^{+/-}$mice. Similar to this study, Moore and colleagues were able to show that intraperitoneal administration of NaHS induced neovascularization in an invivo mouse model using a Matrigel plug assay (269). A previous report showed that genetic deletion/silencing of CSE (another $\mathrm{H}_{2} \mathrm{~S}$-producing enzyme) in the endothelium reduced migration and sprouting of ECs in-vitro, wherein VEGF played a critical mediator (270). In the present study, I observed that PPAR-y and VEGF expressions were significantly downregulated in CBS mice compared to WT mice. I also demonstrated that these effects were mitigated via GYY4137 administration. This suggests that most likely VEGF is regulated via the PPAR-y dependent pathway, further corroborating Biscetti, et al.'s findings wherein they clearly showed that activation of PPAR-y led to endothelial tube formation and induction of VEGF in ECs (323). Similarly, other investigators revealed that inhibiting PDE activity by $\mathrm{H}_{2} \mathrm{~S}$ induces PPAR-y protein and mRNA expressions (331).

$\mathrm{NO}$ is also an endogenous gasotransmitter that, like $\mathrm{H}_{2} \mathrm{~S}$, is involved in vasorelaxation and stimulation of angiogenesis $(346,347)$. HHcy was also found to quench NO (a vasodilator) by the formation of peroxynitrite anion (ONOO) and uncoupling of eNOS, further reducing the bioavailability of NO (348-350). Similarly, I noticed nitrite levels and phosphorylation of eNOS were found to be reduced in CBS mice in comparison to WT mice. eNOS is known to produce NO during angiogenesis via VEGF axis (328); thus, it appears that impaired angiogenesis in HHcy could be due to the reduction of NO bioavailability. In this work, I demonstrated that nitrite levels and 
eNOS activation were reduced in the $\mathrm{CBS}^{+/-}$mice compared to WT mice, and their levels could be recovered via GYY4137 treatment. These findings are also highly consistent with previous reports where $\mathrm{H}_{2} \mathrm{~S}$ was shown to stimulate Akt in endothelial cells, leading to the induction of eNOS through phosphorylation of Ser1177 (activation site) and parallel dephosphorylation of Thr495 (inhibitory site) $(351,352)$.

In conclusion, my work embodies the pro-angiogenic role of the $\mathrm{H}_{2} \mathrm{~S}$ molecule during HHcy condition. I opine that additional pathways might be at work during angiogenesis (97); however, further investigation needs to be undertaken involving similar but not identical scenarios wherein muscle myopathy is the outcome of metabolic abnormalities. In brief, $\mathrm{H}_{2} \mathrm{~S}$ does hold potential ramifications towards developing it as a clinically relevant therapeutic option for chronic conditions that are implicated in a host of inflammatory and cellular stress injury, including the apparent defect in angiogenesis (97). 


\section{CHAPTER V}

\section{HYDROGEN SULFIDE MITIGATES HHCY-MEDIATED SKELETAL MUSCLE ATROPHY VIA JNK/FOXO1 AXIS}

\section{Introduction}

Homocysteine (Hcy), a sulfur-containing non-protein amino acid is generated via onecarbon metabolism in the methionine cycle. Dietary methionine is first converted to $S$ adenosyl methionine (SAM) and then to S-adenosyl homocysteine (SAH) in methionine cycle. SAH is further converted to Hcy which is remethylated to methionine via folate cycle and the cycle continues (353). Under conditions of low cysteine or saturation of Hcy remethylation, Hcy is further metabolized by the transsulfuration pathway to cysteine (2). In the rate-limiting step of the transsulfuration pathway, Hcy is first converted to cystathionine through cystathionine $\beta$-synthase (CBS) where Vitamin B6 (pyridoxine) is an essential co-factor (7). Cystathionine is further transformed to cysteine by cystathionine y-lyase (CSE), which further generate GSH. The normal range of plasma Hcy levels for young adults (0-30 years) is $4.6-8.1 \mu \mathrm{M}$ and for older adults (30 years and above) is $4-15 \mu \mathrm{M}(354)$. In healthy individuals, the generation and elimination of Hcy remain at equilibrium. However, during hyperhomocysteinemia (HHcy), the plasma Hcy levels tend to increase (moderate HHcy: 15-30 $\mu \mathrm{M}$, intermediate HHcy: 30-100 $\mu \mathrm{M}$, and severe HHcy: >100 $\mu \mathrm{M})(7,355)$. Mutations in the CBS gene causes Hcy accumulation in the blood circulation due to disruption of the transsulfuration pathway (356). 
Skeletal muscular atrophy is a pathophysiological condition that arises due to HHcy (357). It causes loss of skeletal muscle mass, leading to muscle weakness, inactivity, and increased mortality $(5,15,20,21,46,66,82)$. The molecular mechanism attributed to muscle atrophy is the upregulation of ubiquitin ligases like RING-finger protein-1 (MURF-1) and muscle atrophy F-box (MAFBx), also known as Atrogin-1, which initiates the degradation of muscular proteins and eventually cell death and muscle mass reduction $(358,359)$. Indeed, several E3 ubiquitin ligases, such as muscle MuRF1, MAFBx, Nedd4.1, TRAF6, and MUSA1, have been identified which mediate degradation of both thick and thin filaments during skeletal muscle atrophy $(214,215)$. Therefore, identification of the precise molecular mechanism(s) as to how these E3 ubiquitin ligases are regulated during $\mathrm{HHcy}$ is essential to devise future preventive strategies.

Forkhead Box (FOX) proteins are a group of transcription factors with a conserved DNA binding domain and are responsible for regulating a number of E3ubiquitin ligases upon a variety of cellular stress responses (216-219). Previous studies showed that growth factor-activated protein kinase B (Akt) and stress-activated c-Jun Nterminal kinase (JNK) have opposing effects on FOX class O (FOXO) (common in mammals): Akt inhibits FOXO activity via preventing its nuclear localization, whereas JNK increases FOXO activity by promoting its import in the nucleus $(216,220-223)$. FOXO1 and 3 are widely expressed across all tissues, FOXO4 is primarily expressed in muscle and kidney tissues, and FOXO6 is expressed in developing brain and liver tissues (360). Post-translational modifications of FOXOs determine its regulation and therefore, its cellular localization (216). FOXO1 has been shown to be the primary transcription factor regulating MuRF1 and Atrogin-1, these two proteins were reported to be highly upregulated during skeletal muscular atrophy (224).

CBS and CSE are the vital enzymes generating $\mathrm{H}_{2} \mathrm{~S}$ from Hcy via the transsulfuration pathway (330). $\mathrm{H}_{2} \mathrm{~S}$ is increasingly being recognized as an important 
signaling molecule in the cardiovascular and nervous systems because of its ability to neutralize a variety of ROS moieties $(63,236,329)$. In fact, patients with a dysregulated Hcy metabolism due to CBS deficiency are likely to be more prone to oxidative stressmediated damages $(361,362)$. Hence, the purpose of this study was to analyze whether Hcy instigates muscle atrophy via upregulation of E3 ubiquitin ligases like MURF-1 and Atrogin-1 through JNK/FOXO1 axis and $\mathrm{H}_{2} \mathrm{~S}$ attenuates these effects. My results suggest that HHcy can induce muscle atrophy via upregulation of Atrogin-1 and MuRF1, whereas $\mathrm{H}_{2} \mathrm{~S}$ could be used as a therapeutic option to restore physiological homeostasis in skeletal muscle during HHcy condition.

\section{Materials and methods}

Animal maintenance and diet protocol. Male wild-type (WT, C57BL/6J) and $\mathrm{CBS}^{+/-}$(B6.129P2-Cbstm1Unc/J 002853) mice were purchased from the Jackson Laboratory (Bar Harbor, ME, USA) (71). All animals were 8-12 weeks-old and maintained in 12:12 h light-dark cycle with regular mouse chow diet in the animal facility of the University of Louisville. All animal protocols and care were carried out according to the guidelines of National Institute of Health (NIH Pub. No. 86-23, revised 1985) and were approved by the Institutional Animal Care and Use Committee (IACUC) of the University of Louisville (KY, USA). Animals were divided into 4

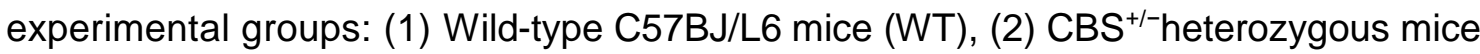
fed with methionine (CBS+Met) (3) NaHS-supplemented wild-type mice (WT+NaHS), and (4) NaHS-supplemented $\mathrm{CBS}^{+/-}+\mathrm{Met}(\mathrm{CBS}+\mathrm{Met}+\mathrm{NaHS})$. Mice were treated with NaHS for 8 weeks $(30 \mu \mathrm{M} / \mathrm{kg} /$ day, I.P. $)$ and fed with a methionine-enriched and lowfolate, low-Vitamin B6, low-B12 diet (Cat. No. TD 97345; Harlan Teklad, Madison, WI, USA) (301-304), while the WT mice were given $0.9 \%$ normal saline (vehicle control) 
and fed with normal chow (Purina Farmer's Exchange, Framingham, MA, USA). Body weights and physical activity monitoring and tissue collection. Body weights were measured at 0 week, 4 weeks and after 8 weeks intervals during $\mathrm{NaHS}$ treatment (Table 4, Appendix I). Further, the animals were routinely inspected for discomfort, body posture, skin integrity (injury), and fur appearance to monitor their physical activity. At the end of the experiment, animals were euthanized by using $2 \mathrm{X}$ tribromoethanol (TBE), and both blood and muscle samples were collected for further analysis.

Genotype analysis of the heterozygous $\mathrm{CBS}^{+-}$mouse. After purchasing, mice were cross-bred, yielding around $10 \% \mathrm{CBS}^{-/-}, 60 \% \mathrm{CBS}^{+/-}$, and $25 \% \mathrm{CBS}^{+/+}$. For genotyping, tail samples were collected, and genotypic analysis was performed using PCR by targeted disruption of the CBS gene at loci, as shown in Fig. 18A. The PCR products were run on $1.2 \%$ agarose gel (prepared in TAE buffer, $\mathrm{pH} 8.4$ ) in the presence of ethidium bromide and the images recorded in a gel documentation system (305). $\mathrm{CBS}^{+/-}$heterozygote gene-positive mice produced two bands (450 and $308 \mathrm{bp}$ ), while $\mathrm{CBS}^{+/+}$mice represented only one band (308 bp).

Cell culture and treatments. C2C12 cells (immortalized mouse myoblast cell line, ATCC) were cultured in Corning® T-75 flasks in ATCC-formulated DMEM supplemented with $10 \%$ Fetal Bovine Serum (FBS), $0.1 \%$ penicillin and streptomycin (P/S) at $37^{\circ} \mathrm{C}$ with $5 \%$ $\mathrm{CO}_{2}$. The $\mathrm{C} 2 \mathrm{C} 12$ cells were grown to $80 \%$ confluence and were plated for 4 different

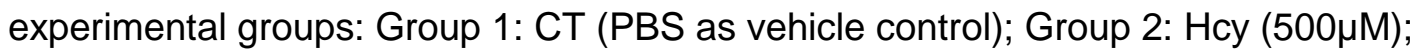
Group 3: Hcy+NaHS $(250 \mu \mathrm{M})$ and Group 4: NaHS. In this study, we used 1mM Hcy and $250 \mu \mathrm{M}$ NaHS concentrations for the individual treatments $(20,246)$. A stock solution of Hcy, NaHS were prepared by directly dissolving in basal DMEM medium (serum-free media). Following $24 \mathrm{~h}$ of treatment as mentioned earlier, cells were processed for further biochemical analysis. 
Reagents and antibodies. Dulbecco's Modified Eagle's Medium (DMEM), fetal bovine serum (FBS) were purchased from American Type Culture Collection (Manassas, VA, USA) and trypsin EDTA was from VWR (Radnor, PA, USA). ECL reagent and polyvinylidenedifluoride (PVDF) membrane were from Bio-Rad (Hercules, CA, USA). All other reagents and chemicals were ordered from Sigma-Aldrich or available highest grade.

Primary antibodies for Western blot analysis: rabbit anti-FOXO1A (1:1000, Abcam, Cat \# ab12161), rabbit anti-p-FOXO1A (Thr24) (1:1000, Millipore, Cat \#2599), mouse anti-GAPDH (1:10000, Millipore, Cat \# MAB374). Atrogin-1 (1:1000, Abcam Cat \# ab92281), myosin heavy chain-I (MHC-I) (1:1000, Abcam Cat \# ab11083), MuRF-1 (1:1000, Abcam Cat \# ab172479) and Laminin (1:1000, Abcam Cat \# ab11575). Secondary antibodies for Western Blot Analysis: mouse anti-rabbit (1:2000), rabbit antimouse (1:2000) were ordered from Santa Cruz Biotechnology (Dallas, TX, USA). Alexa Fluor 488 donkey anti-mouse (Cat \# R37114), Alexa Fluor 488 donkey anti-rabbit IgG $(H+L)(1: 1000$, Cat \# A21206), Alexa Fluor 546 donkey anti-mouse $\lg G(H+L)(1: 1000$, Cat \# A10036), Alexa Fluor 546 donkey anti-rabbit lgG $(H+L)(1: 1000$, Cat \# A10040) were ordered from Invitrogen (Eugene, OR, USA) Immunocytochemistry (ICC). For ICC, $\mathrm{C} 2 \mathrm{C} 12$ cells were grown in chamber slides (LabTek II) and treated as mentioned above. After $24 \mathrm{~h}$ of treatment, cells were washed 2 times with 1X phosphate buffer saline (PBS) and fixed with 4\% paraformaldehyde (PFA) diluted in 1 X PBS for 10 minutes at room temperature. The cells were permeabilized and blocked at the same time with a solution of $2 \%$ bovine serum albumin (BSA) and $0.5 \%$ Triton X-100 in 1X PBS for 30 minutes at room temperature. The cells incubated with primary antibodies diluted in $0.1 \%$ BSA and $0.5 \%$ Triton X-100 in 1 X PBS at the concentrations as mentioned above and kept for overnight at $4^{\circ} \mathrm{C}$. The next day the cells were washed 3 times with 1 X PBS and then incubated with secondary antibody using 
the concentrations as mentioned above for $1 \mathrm{~h}$ at room temperature. After washing the cells 5 times with 1 X PBS after secondary antibody incubation, they were mounted with mounting media containing DAPI (Vectashield Cat \# H-1200) and viewed under the confocal microscope (Olympus FluoView1000, Pittsburgh, PA, USA). Immunohistochemistry (IHC). For IHC, I used cryo-tissue sections of the gastrocnemius muscle $(7 \mu \mathrm{m})$ which were labeled for immunofluorescence following the standard protocol. Briefly, first, the tissue sections were fixed in $4 \%$ paraformaldehyde and permeabilized with $0.25 \%$ Triton X-100 in PBS. Then, the sections were incubated overnight in primary antibodies at $4{ }^{\circ} \mathrm{C}$, and after that, secondary antibodies labeled with either Alexa Fluor-488 or 594 (Invitrogen) appropriate to the primary antibody species were applied. Sections were cover-slipped with ProLong ${ }^{\mathrm{TM}}$ Gold Antifade Mountant. Stained images were visualized and analyzed for fluorescence intensity under an EVOS $^{\text {TM }}$ FL Auto Imaging System (Thermo Fisher Scientific, Waltham, MA, USA) using an appropriate filter. To study the cross-sectional area in the gastrocnemius muscle from each group, I analyzed 30-40 fields/mouse.

Western blot analysis. First, the cells or tissues were mixed with cold RIPA lysis buffer with protease inhibitor cocktail, phenylmethylsulfonyl fluoride (PMSF) and phosphatase inhibitor (sodium orthovanadate) and then sonicated employing the Sonifier 450 (Branson Ultrasonics, Danbury, CT, USA) using 3 x $5 \mathrm{sec}$ at setting "3". After that the suspensions were centrifugated at $17,400 \times \mathrm{g}$ for 20 minutes at $4^{\circ} \mathrm{C}$. The supernatants were collected in fresh tubes and were stored at $-80^{\circ} \mathrm{C}$ until further use. Protein concentration was determined using Bradford assay to ensure that the proteins were loaded in equal concentrations. Protein lysates were subjected to $8-12 \%$ sodium dodecyl sulfate-polyacrylamide gel electrophoresis (SDS-PAGE) in a tris-glycine-SDS buffer for proper protein separation and transferred on a polyvinylidene difluoride (PVDF) membrane electrophoretically overnight at $4^{\circ} \mathrm{C}$. The membrane was first blocked using 
$5 \%$ milk in $1 \mathrm{X}$ tris-buffer saline and Tween 20 (TBS-T) for $1 \mathrm{hr}$ at $4^{\circ} \mathrm{C}$ and then incubated with the primary antibodies at the concentrations as mentioned above for overnight at $4^{\circ} \mathrm{C}$. After primary antibody incubation, the membranes were washed 3 times with $1 \mathrm{X}$ TBS-T followed by incubation with secondary antibody conjugated with HRP for $1 \mathrm{hr}$ at room temperature. Membranes were again washed 3 times with TBS-T, then developed using ECL Luminata Forte reagent (Millipore, Temecula, CA, USA) and imaged using a Chemidoc (Bio-Rad, Hercules, California, USA). Band intensity was determined using densitometry analysis using Image Lab ${ }^{\text {TM }}$ Software (Bio-Rad, Hercules, CA, USA). Total Homocysteine measurement. The total thcy levels were measured from plasma of experimental mice using homocysteine assay kit (Crystal Chem, IL, USA) as per manufacturer's instructions.

Muscle fatigability tests. Muscle fatigability test was developed from recommendations listed in the Resource Book for the Design of Animal Exercise Protocols by the American Physiological Society (APS) with minor modifications. First, one mouse at a time was allowed to swim for 10 mins across 4 different days for acclimatization to the environment. I used a swimming tub with water temperature usually between 32$36^{\circ} \mathrm{C}$. The depth of water was maintained at a minimum of $30 \mathrm{~cm}$ so that mice couldn't touch the bottom with $10-15 \mathrm{~cm}$ distance left from the top to prevent animals from climbing or jumping out. On the final day, each mouse was placed in the water to swim to check their maximum swimming capacity (Table 5, Appendix I). To monitor their live motion, I used Clever Systems (Reston, VA, USA) in the previously discussed method (46). If the mice discontinue swimming for 2 secs, they were gently nudged to promote their movement, and if they were drowning in the water, then they were immediately taken out as per protocol.

To determine muscle grip strength of experimental mice, I used Rotarod instrument (San Diego Instruments, San Diego, CA, USA) and Grip strength test meter 
(Bioseb, Pinellas Park, FL, USA) $(364,365)$. Briefly, Rotarod performance was done by placing them on the rotarod and allowing them to run at a constant low speed (12 RPM) for 5 mins across 4 different days (acclimatization steps). Following acclimatization, on the final day mice were placed on the rotarod (12 RPM) and allow to run until they fall off from the apparatus (time was recorded) (Table 5, Appendix I). The grip strength of both forelimbs and hindlimbs were measured by using a grip strength meter. Each mouse was held by the base of the tail and placed in front of the grasping grid. Once the mouse grasped the grid, it was slowly pulled back until the pulling force overcame the mouse's grip strength. This process was repeated 5 times for each mouse with 15 mins gap between each repeat and all measurements of grip strength/body weights were recorded (Table 5, Appendix I).

Statistical analysis. All values were expressed as mean \pm s.e.m. One-way or two-way ANOVA was conducted for the statistical analyses for the different treatment groups using GraphPad Prism (Ver. 7) software. For all in-vivo experiments mice number $(n)=4$ 5 , in each group, and for all exercise capacity test, $(n)=9-11$ mice were used in each group. The significance threshold was set at $p<0.05$. A minimum of 3 technical replicates were conducted for each protein expression analysis and ICC experiment.

\section{Results}

HHcy causes skeletal muscle atrophy in CBS+Met mice. In this study, I noticed $C B S+$ Met mice had significantly low body weights most likely because of excessive muscle wasting in comparison to WT mice (Fig. 18A and 18B). Although I didn't observe any changes in tibial length between each group of experimental mice, gastrocnemius and quadriceps muscles weights were significantly reduced in CBS+Met compared to WT mice (Fig. 18C and 18D). Although not significant, I did notice a reduction of tibialis anterior (TA), extensor digitorium longus (EDL) and soleus muscles weights in CBS+Met 
mice compared to WT mice. After administration of NaHS for 8 weeks, I noticed an improvement in overall body weights and muscle mass as shown in Fig. 18C and 18E. I also noticed tHcy levels in plasma were significantly increased in CBS+Met than WT mice and was similar in NaHS treated CBS+Met mice (Fig. 18F).

A

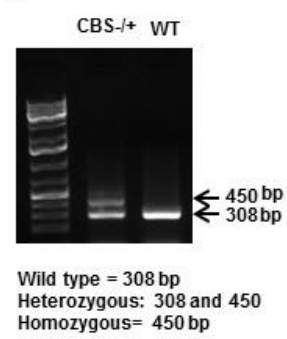

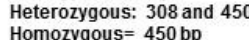
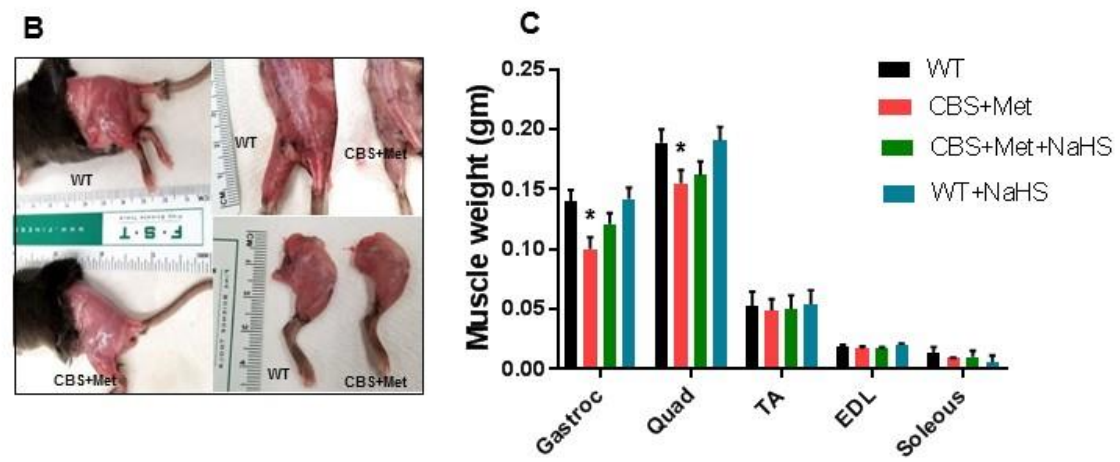

$$
\text { D }
$$

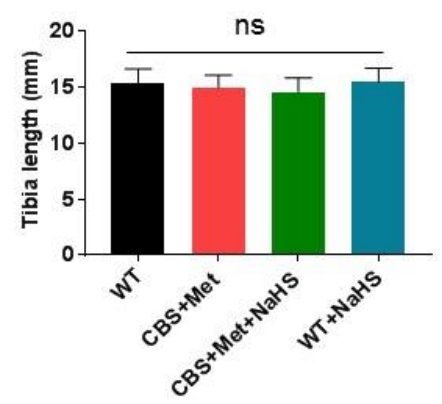

E

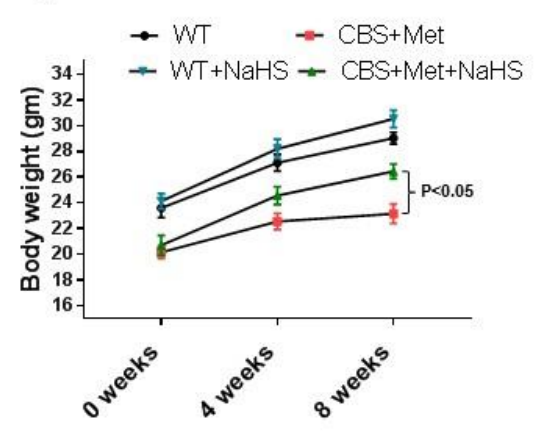

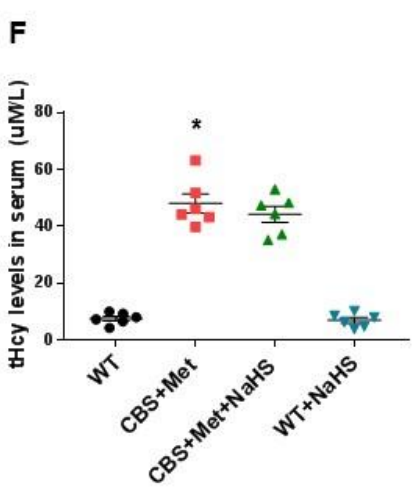

Figure 18: Genotypic and phenotypic characteristics of experimental mice groups. (A) Genotyping of CBS+/- and WT mice; (B) morphological difference of skeletal muscle in hindlimbs between CBS+Met and WT mice; (C) difference in various muscle weights such as gastrocnemius (gastroc), quadriceps (Quad), tibialis anterior (TA), extensor digitorium longus (EDL) and soleus, where mice number $(n)=4$ in individual group; (D) Tibia length measurements, where mice number $(n)=4$ in individual group; (E) Body weight measurements, where mice number $(n)=4$ in individual group; $(F)$ total homocysteine (tHcy) measurements, where mice number $(n)=4$ in individual group. The 
overall difference between the experimental groups was determined by one-way or twoway ANOVA, and a Tukey's post hoc analysis was run when overall statistically significant difference occurred in group means. Data are shown as mean \pm s.e.m. and statistical difference ${ }^{*} p<0.05$ vs. WT and ${ }^{\#} p<0.05$ vs. CBS+Met.

NaHS treatment improves muscle fatigability in $C B S+M e t$ mice. To measure $\mathrm{HH} c y$ effect on muscle fatigability, I performed swimming capacity test for all experimental groups. I noticed that CBS+Met mice moved less distance and spent less time in swimming, whereas NaHS supplementation improved their capacities significantly (Fig. 19A and 19B). Similar findings were also observed for the muscle grip strength test as shown by less latency to fall from rotarod and grip strength/BW in CBS+Met as compared to WT mice. After 8 weeks of NaHS administration, these effects were substantially improved in CBS+Met mice (Fig. 19C and 19D). 
A

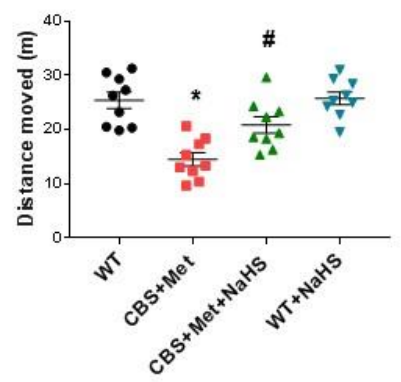

Swimming Performance Test

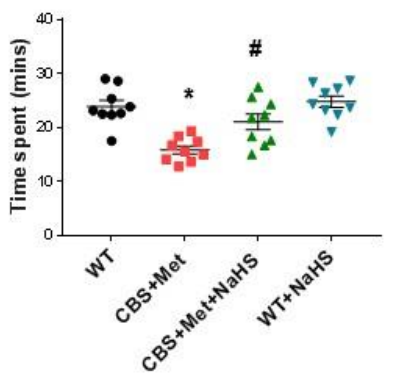

B

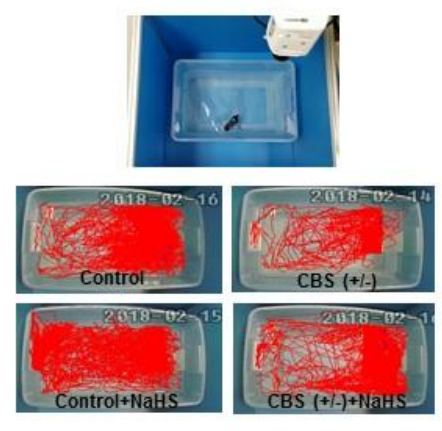

D

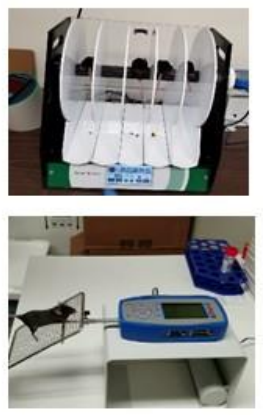

C

Grip Strength Test
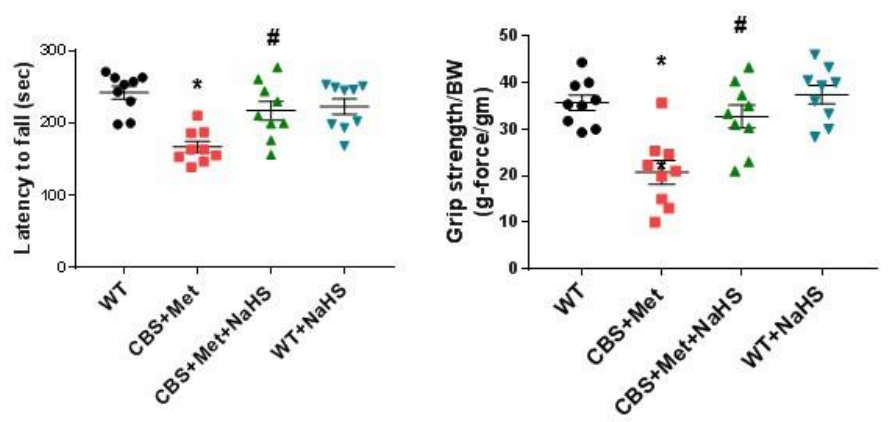

Figure 19: NaHS treatment improves muscle fatigability in CBS+Met mice compared to WT mice. (A) Measurements of muscle fatigability was done by swimming test, in the left side shows total distance moved and on the right side shows total time spent during the swimming performance test; (B) Images captured during live recording of swim test (top), representative animal tracings were shown after motion performance in swimming test for each group (bottom); (C) Images showing results of grip strength using rotarod performance test (left) and grip strength test (right). (D) Images captured in rotarod (top) and Bioseb grip strength meter (bottom) during grip strength test. The overall difference between the experimental groups was determined by one-way or two-way ANOVA, and a Tukey's post hoc analysis was run when overall statistically significant difference occurred in group means. Data are shown as mean \pm s.e.m. and mice number $(n)=9$ mice and ${ }^{*} p<0.05$ vs. WT and ${ }^{*} p<0.05$ vs. CBS+Met. 
HHcy induced morphological changes in skeletal muscle in-vivo. To understand the effect of HHcy on morphological changes in the muscle fibers, I analyzed the crosssectional areas (CSAs) by laminin staining. Results of the staining showed a significant reduction of CSAs in gastrocnemius muscle fibers isolated from CBS+Met in comparison to WT mice (Figure 20A and 20B). Although not statistically significant, I observed an improvement of CSAs of skeletal muscle fibers in CBS+Met mice after NaHS treatment for 8 weeks.

A

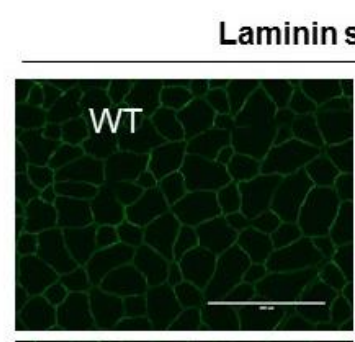

\section{WT+NaHS}

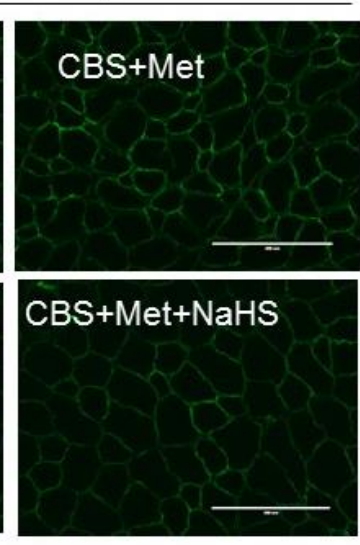

B

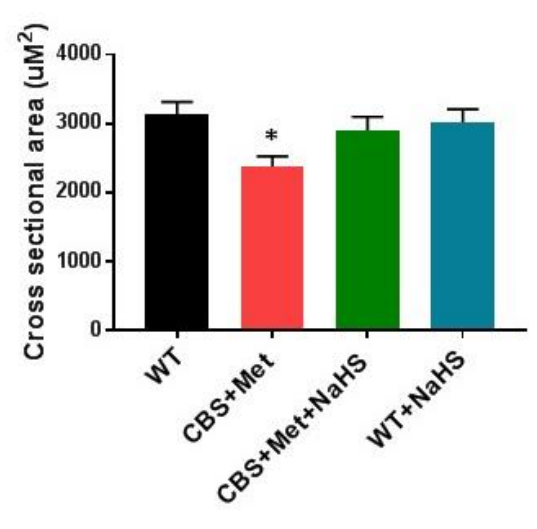

Figure 20: HHcy reduces cross-sectional areas (CSAs) of skeletal muscle fibers in CBS+Met mice compared to WT mice. (A) Laminin staining showing cross-sectional areas (CSAs) measurements are low in GA muscle of CBS+Met mice compared to WT

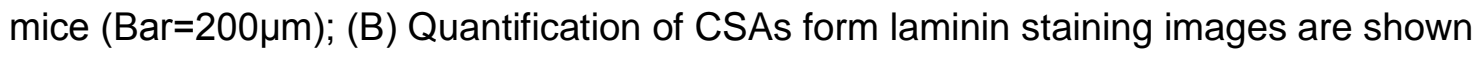
on the right. To study the CSAs of the gastrocnemius muscle fiber from each group, I analyzed 30-40 fields/mouse. Representative image from each group of mice is shown here. The overall difference between the experimental groups was determined by oneway ANOVA, and a Tukey's post hoc analysis was run when overall statistically significant difference occurred in group means. Data are shown as mean \pm s.e.m. and mice number $(n)=4$ and ${ }^{*} p<0.05$ vs. WT. 
To check whether HHcy induces fibrosis in skeletal muscle fibers, I performed H\&E and Masson trichrome staining in muscle CSAs of gastrocneous muscle. Results revealed a higher level of fibrosis and collagen deposition in muscle CSAs derived from CBS+Met as compared to WT mice. Interestingly, treatment with NaHS for 8 weeks was found to alleviate these effects in CBS+Met mice (Fig 21A-21C).

A
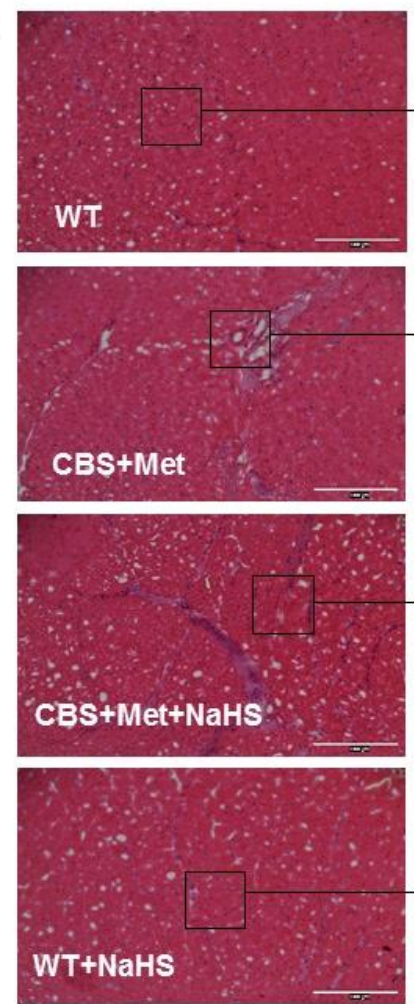
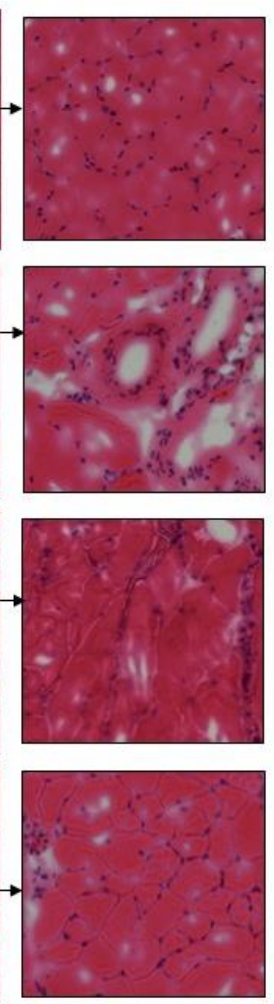

C
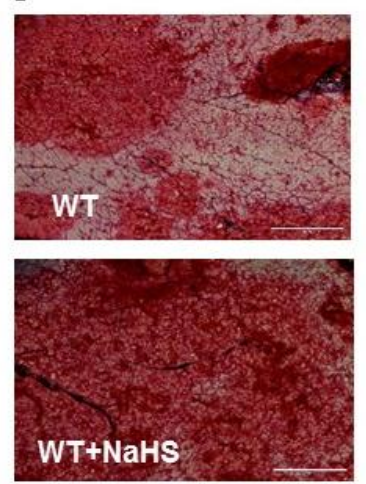

CBS+Met+NaHS
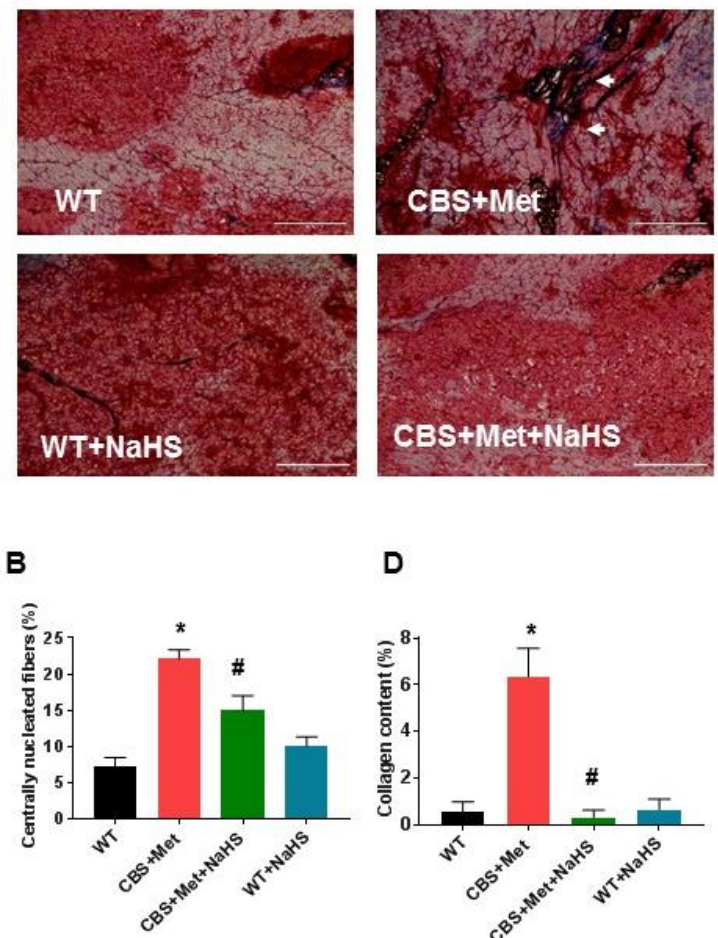

D

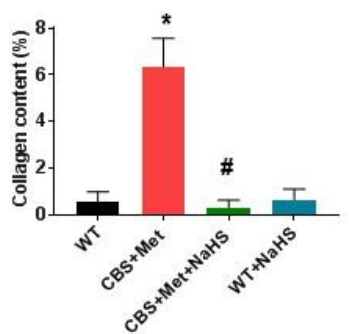

Figure 21: HHcy-induced fibrosis and collagen deposition in skeletal muscle in-vivo. (D) H\&E staining in cross sectional areas of gastrocnemius muscle showing morphological differences of muscle fibers between CBS+Met and WT mice, images are taken using EVOS $尺$ FL Auto Cell Imaging System where mice number $(n)=4$. (Bar=400 $\mu m)$, Representative areas from H\&E staining images are zoomed in to show more clear view; (E) Quantification of regenerated muscle fibers as quantified by centrally located nuclei from H\&E staining images; $(F)$ Masson's Trichrome staining in cross sectional areas 
(CSAs) of gastrocnemius muscle showing blue color stain (white arrowheads) as collagen deposition in the muscle of CBS+Met mice compared to WT mice. Images are taken using EVOS® FL Auto Cell Imaging System where mice number $(n)=4-5$.

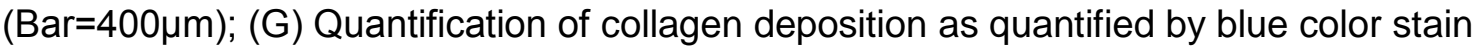
from Masson's Trichrome staining images. The overall difference between the experimental groups was determined by one-way ANOVA, and a Tukey's post hoc analysis was run when overall statistically significant difference occurred in group means. Data are shown as mean \pm s.e.m. and mice number $(n)=4-5$ and ${ }^{*} p<0.05$ vs. WT and ${ }^{\#} \mathrm{p}<0.05$ vs. CBS+Met.

Effect of Hcy on Akt-phophorylation, and subcellular localization of FOXO1. To study the effect of HHcy on Akt-phosphorylation, I did Western blots analysis; the results showed that Hcy treatment significantly reduced Akt-phosphorylation in $\mathrm{C} 2 \mathrm{C} 12$ cells as compared to vehicle controls, whereas this effect was successfully mitigated via NaHS administration (Fig. 22A).

As I noticed Akt-phophorylation is downregulated and JNK-phosphorylation is upregulated after Hcy treatment in $\mathrm{C} 2 \mathrm{C} 12$ cells (Figure 22A and 12), I tested the subcellular localization of FOXO1 using ICC analysis. My confocal images showed that p-FOXO1 is primarily localized in the nucleus and FOXO1 is primarily expressed in the cytoplasm in the cells across all the treatment groups (Fig. 22B). However, I observed that the pFOXO1/FOXO1 ratio is high for Hcy treated $\mathrm{C} 2 \mathrm{C} 12$ cells compared to controls and this effect was reversed via NaHS supplementation (Fig. 22B). 


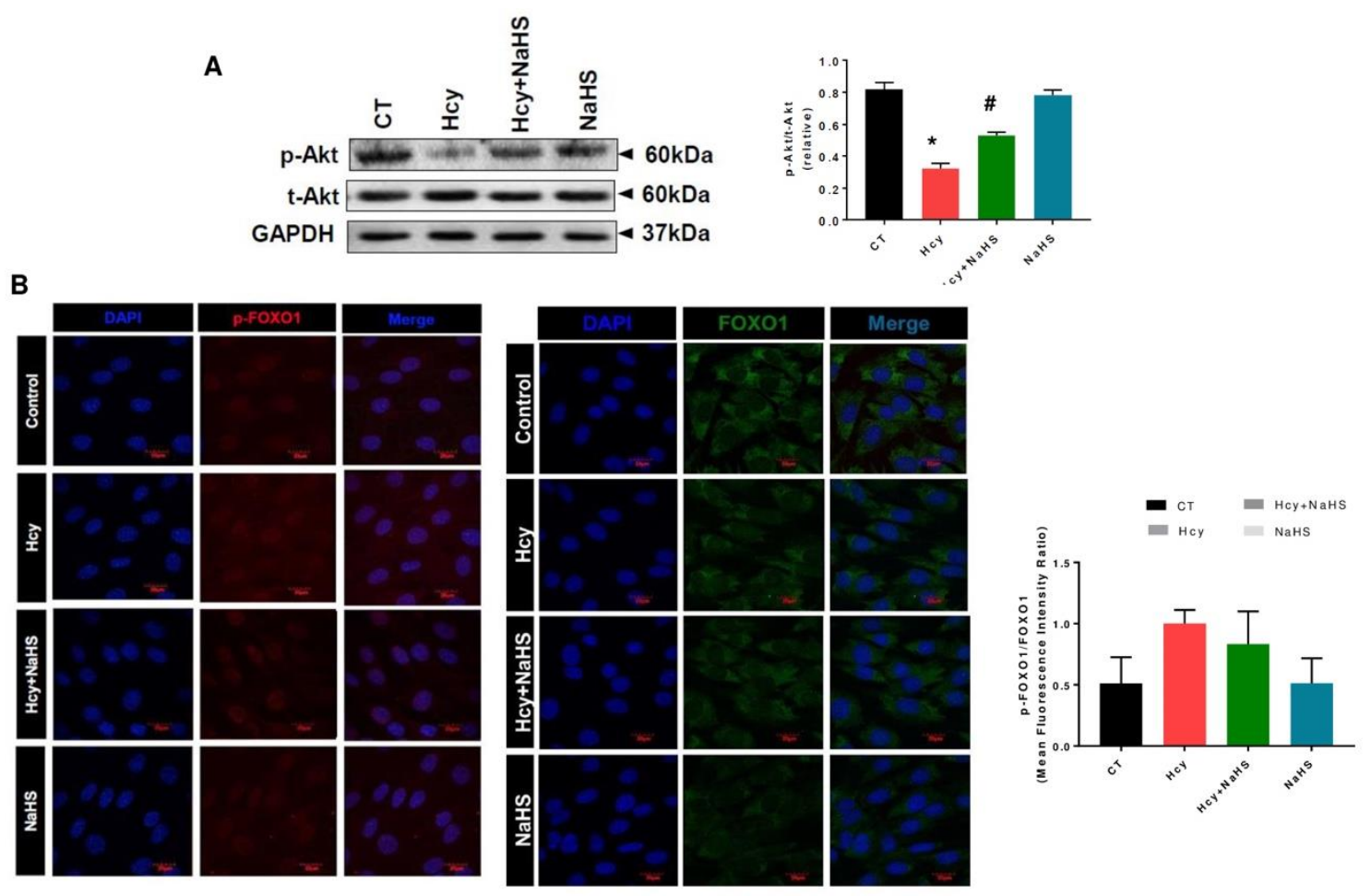

Figure 22: Hcy attenuates Akt-phosphorylation and induced nuclear localization of pFOXO1 in $\mathrm{C} 2 \mathrm{C} 12$ cells. (A) Western blots analysis showing Hcy treatment reduced Aktphosphorylation, whereas NaHS treatment mitigates this effect. Densitometric measurement of Akt-phosphorylation from above Western blots image is shown on the right, where $n=3$ technical replicates; (B) Representative images showing intracellular localization of p-FOXO1 and FOXO1 in the left. The p-FOXO1/FOXO1 ratio based on fluorescent intensity from ICC images are shown on the right, where $n=3$ technical replicates. Cells were observed and imaged under the confocal microscope at 100X magnification. Quantification of fluorescent intensity were shown on the right. The overall difference between the experimental groups was determined by one-way ANOVA, and a Tukey's post hoc analysis was run when overall statistically significant difference occurred in group means. Data are shown as mean \pm s.e.m., and ${ }^{*} \mathrm{p}<0.05$ vs. CT and \#p<0.05 vs. Hcy. 
Hcy enhanced Atrogin-1 and MuRF-1 expression in the skeletal muscle. I noticed the expression of Atrogin-1 and MuRF-1 were higher in Hcy treated C2C12 cells in comparison to vehicle controls, and this effect was attenuated by NaHS treatment (Fig. 23A). Similar findings were observed in muscle collected from experimental mice (Fig. 23B). In addition, IHC experiments confirmed higher MuRF-1 and reduced myosin heavy chain type-I (MHC-I) expression in skeletal muscle of CBS+Met mice as compared to WT mice, and this effect was alleviated by NaHS treatment (Fig. 24A-C). The Western blot results also confirmed a similar association as seen in IHC staining (Figure 24D and E).
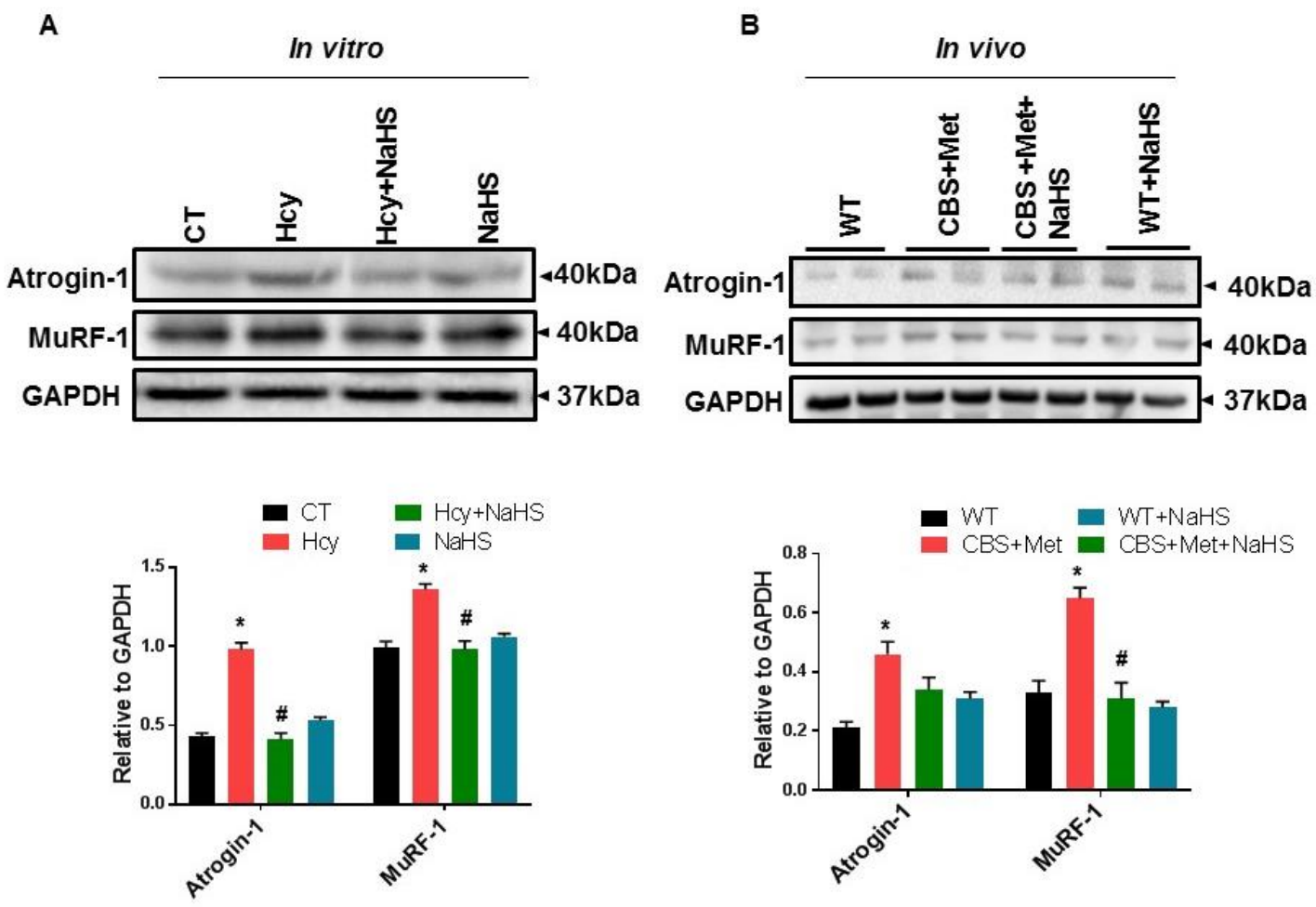

Figure 23. Hcy enhanced Atrogin-1 and MuRF-1 expressions in the skeletal muscle. (A) Western blots data showing expression of Atrogin-1 and MuRF-1 were elevated in C2C12 cells after Hcy treatment compared to controls. Densitometric measurements of Atrogin-1 and MuRF-1 expression from above Western blots images are shown on the 
bottom, where $n=3$ technical replicates. Data are shown as mean \pm s.e.m., where $n=3-4$ biological replicates and ${ }^{*} p<0.05$ vs. CT and ${ }^{\#} p<0.05$ vs. Hcy. (B) Western blots data showing expression of Atrogin-1 and MuRF-1 were elevated in the muscle of CBS+Met mice compared to WT mice. (H) Densitometric measurement of Atrogin-1 and MuRF-1 expression from above Western blots images is shown on the bottom, and where mice number $(n)=4$. The overall difference between the experimental groups was determined by one-way or two-way ANOVA, and a Tukey's post hoc analysis was run when overall statistically significant difference occurred in group means. Data are shown as mean \pm s.e.m. and ${ }^{*} p<0.05$ vs. WT and ${ }^{\#} p<0.05$ vs. CBS+Met.
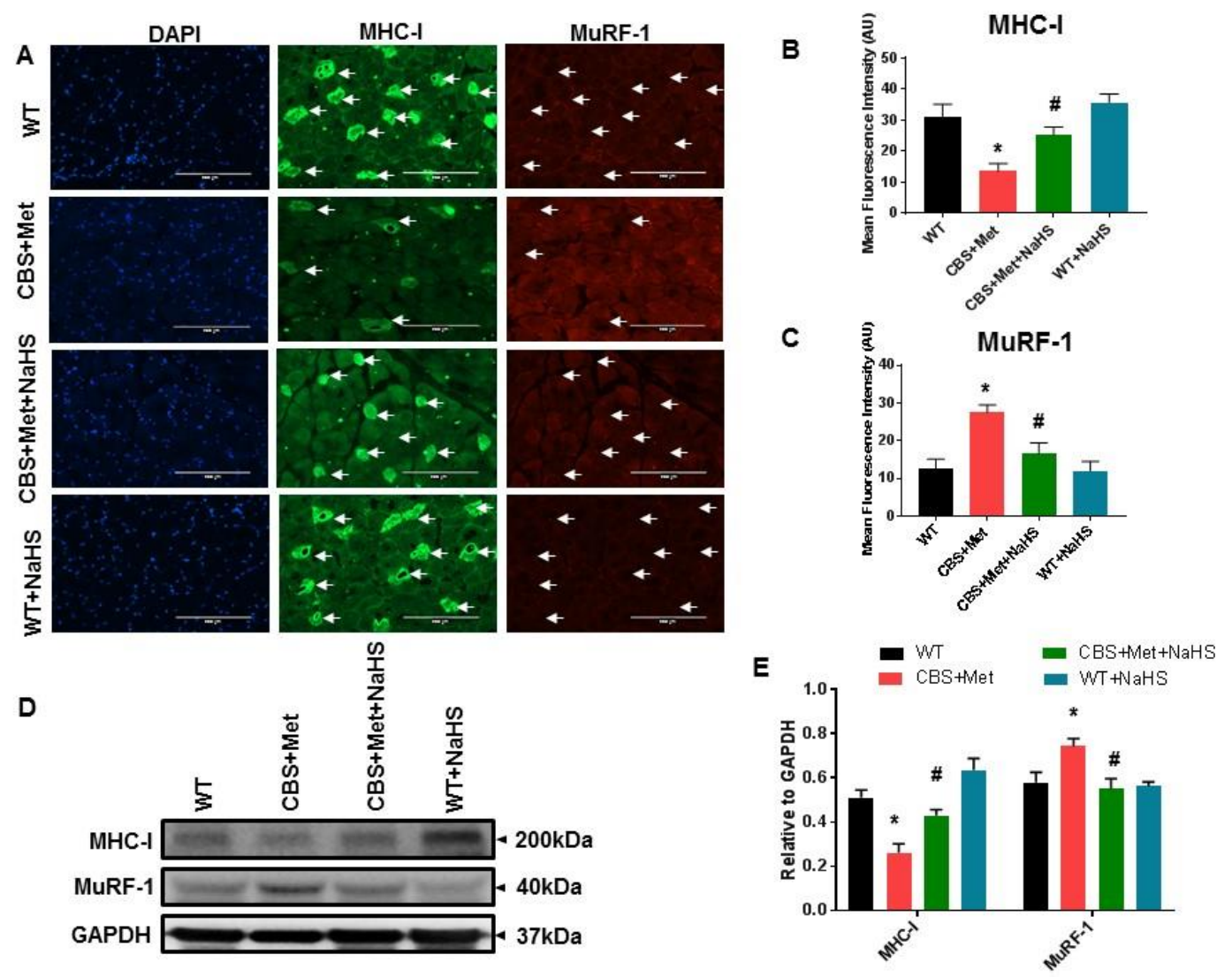

Figure 24. High Hcy mediates elevated expression of MuRF1 and degradation of its targets protein MHC-1 in skeletal muscle of CBS mice compared of WT mice. (A) 
Immunohistochemistry analysis showing representative images of elevated expression of MuRF1 and reduced expression of MHC-I in tissue cross-section of the GA muscle in CBS+Met mice compared to WT mice. The arrows indicate the respective expression levels of MuRF-1 (low) and MHC-1 (high) in the fiber content of the GA muscle

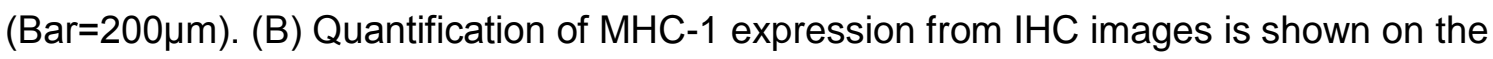
right and where mice number $(n)=4$. (C) Quantification of MuRF-1 expression from IHC images is shown on the right, and where mice number $(n)=4$. (D) Western blots data showing protein expression of MHC-1 and MuRF-1 in the muscle of CBS+Met mice compared to WT mice. (E) Densitometric measurement of MHC-I and MuRF-1 from above Western blots images is shown on the right, and where mice number $(n)=4$. The overall difference between the experimental groups was determined by one-way or twoway ANOVA, and a Tukey's post hoc analysis was run when overall statistically significant difference occurred in group means. Data are shown as mean \pm s.e.m. and ${ }^{*} \mathrm{p}<0.05$ vs. WT and ${ }^{\#} \mathrm{p}<0.05$ vs. CBS+Met.

\section{Discussion}

Skeletal muscle is the largest organ in the human body and makes up almost $40 \%$ of the body's weight (351). Muscle atrophy can occur due to result in the reduction of muscle mass, structural, and metabolic alteration of intrinsic muscle cells (138). Although HHcy was reported to cause muscle atrophy, the precise molecular mechanism of this effect was not identified before. In this study, I observed a reduction of body weights in HHcy mice (genetically engineered heterozygous CBS deficient mice) compared to age matched WT mice (Fig. 18E). As I also noticed their tibia lengths were similar with WT mice and muscle masses in hindlimb were lower in comparison to WT mice (Fig. 18C), suggesting that overall reduction of body weight may be at least partly due to the reduction of muscle mass (351). The muscle fiber is bound to connective 
tissues, nervous tissues and blood vessels for oxygen and nutrient supply $(366,367)$, so reduction of muscle mass may impair its functional capabilities. Indeed, I noticed that CBS mice have significantly poor performance in swimming and grip strength test compared to WT mice (Fig. 19). Interestingly, I also identified that NaHS administration could successfully mitigate these effects. The basic unit of the muscle is the muscle fiber, so any structural or morphological changes in muscle fiber can play havoc with it functional capabilities (368). Muscle atrophy is most often associated with fibrosis in muscle fibers, excessive accumulation of extracellular matrix (ECM) components, particularly collagen (369). This study showed that HHcy could actually significantly reduce CSAs, and induce fibrosis and collagen deposition in muscle fibers of CBS mice compared to WT mice and these effects of HHcy were improved upon NaHS administration (Fig. 20 and 21). This study is similar to previous study by Kanwar et al. [1976] found that HHcy can cause focal fragmentation, disruption and smearing of the Zdiscs, increased collagen deposition in the besal lamina and disorganization of the myofilaments in the skeletal muscles (14)

To further characterize underlying molecular changes of HHcy-mediated muscle atrophy, I analyzed intracellular localization of p-FOXO1 and FOXO1 by ICC experiment. Regulation of the subcellular localization of FOXO1 is critical to its transcriptional activity (354-356). My findings provide a new insight into the molecular background of the effect of HHcy in induced nuclear translocation of p-FOXO1. I observed induction of p-FOXO1 expression and its nuclear localization upon treatment with Hcy and mitigated upon NaHS treatment (Fig. 22) led us to conclude that possibly Hcy stabilizes FOXO1 in the nucleus and thus allows it to carry on its transcriptional activity. Recent studies indicate that JNK is responsible for FOXO activation under stress conditions $(357,358)$. As I also found high Hcy can activate JNK (chapter IV, Fig. 12), suggesting that JNK may also play a role in regulating nuclear stabilization of FOXO1. When FOXO1 is 
phosphorylated through Akt on $S^{256}$, it creates a 14-3-3 binding site (370), that masks the nuclear localization signal (NLS) and prevents nuclear translocation, thereby inhibiting the activities of FOXO1 (371-373). However, during HHcy condition, Akt is downregulated, hence in addition to JNK pathway, reduced Akt-phosphorylation in C2C12 cells after Hcy treatment can stabilize FOXO1 inside the nucleus thus allowing them to carry their transcriptional activity $(374,375)$.

Previous results suggested the role of FOXO1 transcription factors in the regulation of E3-ubiquitin ligases like Atrogin-1, and MuRF1 (376-379). Similarly, my findings suggested that expression of both MuRF1 and Atrogin-1 were upregulated in both in-vitro and in-vivo models and NaHS administration mitigated these effects. Together these results suggest that upregulation of these E3 ubiquitin ligases may be mediated through the FOXO1 transcription factors, which were found to be involved in most forms of atrophy $(377,380-384)$. The overall results from this study demonstrate that HHcy-mediated oxidative/ER-stress upregulates MuRF1 and Atrogin-1 expression through JNK-dependent FOXO1 activation, which may degrade some vital proteins in muscle, such as MHC-I and MyoD $(385,386)$. Indeed, I noticed that expression of MHC-I was decreased in skeletal muscle of CBS+Met mice (Fig. 24), which indicates that higher expression of MuRF-1 could trigger muscle atrophy via proteasomal degradation of its target proteins such as MHC-I (387). In conclusion, I have elucidated a well-defined signaling pathway for the upregulation of E3 ubiquitin ligases in HHcy-mediated skeletal muscle atrophy through activation of FOXO1 via JNK phosphorylation. I have also provided important data regarding potential mechanistic role $\mathrm{H}_{2} \mathrm{~S}$ in mitigation of $\mathrm{HHcy}$-mediated skeletal muscle atrophy. This study indicates that $\mathrm{H}_{2} \mathrm{~S}$ could be developed as a potential therapeutic target in various forms of musclulopathies wherein HHcy is linked with metabolic dysfunction. 


\section{CHAPTER VI}

\section{SUMMARY}

HHcy is a well-accepted risk factor for vascular diseases (136), and several studies reported that HHcy conditions lead to skeletal muscle weakness and functional impairment $(14,19,20)$. Results from the present study demonstrate that HHcy due to $\mathrm{CBS}^{+/-}$deficiency displays decreased bodyweight, angiogenesis and skeletal muscle mass, which ultimately lead to muscle myopathy. This is similar to neurological disorders such as amyotrophic lateral sclerosis (ALS), and multiple sclerosis, causing muscle degeneration and are also associated with HHcy (15). My dissertation elucidates the potential molecular mechanisms as to how HHcy mediates skeletal muscle myopathy via induction of oxidative and ER-stress responses, apoptosis, atrophy and reduction of angiogenesis (Fig. 25). This study also identified the apparent beneficial effects of $\mathrm{H}_{2} \mathrm{~S}$ on mitigation of HHcy effects on skeletal muscle dysfunction.

In chapter IV, I explored that high Hcy can induce intracellular oxidative and ERstress responses in skeletal muscle. Interestingly, I found that these cellular stress responses were successfully mitigated via NaHS intervention. Moreover, I found that HHcy induced JNK phosphorylation in both in-vitro and in-vivo models, however, I did notice that NaHS could not mitigate JNK-phosphorylation in the in-vitro model, but it could reduce JNK-phosphorylation in the in-vivo model. To my best knowledge, the possible explanation for this observed difference is in the in-vitro settings, I only studied a single readout (24 h post-HHcy), unlike the in-vivo $\mathrm{CBS}^{+/-}$model wherein I was able to 
observe the continuous or prolonged effect of the NaHS mediated phospho-JNK status. Previous studies have revealed that JNK-phosphorylation was able to activate c-Jun which further regulated the expression of several pro-inflammatory and proapoptotic genes (283-291). Indeed, my data showed that the apoptosis rate was induced after Hcy treatment in $\mathrm{C} 2 \mathrm{C} 12$ cells and it was mitigated by the NaHS supplementation.

Furthermore, I found inflammatory cytokines IL-6 and TNF- $\alpha$ were higher in plasma from $\mathrm{CBS}^{+/-}$as compared to wild-type mice; however, this effect was not mitigated via NaHS administration. The overall results from this chapter established the molecular mechanisms of the pathophysiological effects of HHcy in skeletal muscle myopathy via inducing cellular stress responses.

In chapter V, I elucidated that HHcy can inhibit angiogenesis via PPAR $\gamma /$ VEGF axis in a hindlimb ischemia model. This study showed that HHcy reduced the HIF1 $\alpha$, VEGF and p-eNOS levels in skeletal muscle after 21 days of chronic ischemia (generated via femoral artery ligation; FAL) in $\mathrm{CBS}^{+/-}$mice compared to WT mice. Additionally, I also noticed that plasma nitrite levels were low in $\mathrm{CBS}^{+/-}$mice in comparison to WT mice after 21 days of ischemia, suggesting that HHcy may reduce the bioavailability of $\mathrm{NO}$ during angiogenesis in $\mathrm{CBS}^{+/-}$mice compared to WT mice. Interestingly, I also observed GYY4137 (another donor of $\mathrm{H}_{2} \mathrm{~S}$ ) supplementation mitigates this $\mathrm{HHcy}$ effect in $\mathrm{CBS}^{+/-}$mice. Furthermore, I identified the collateral vessel densities, and blood flow rates were lower in the hindlimb of $\mathrm{CBS}^{+/-}$mice after 21 days of ischemia compared to WT mice, and these effects were also attenuated via GYY4137 treatment, suggesting that exogenous supplementation of GYY4137 can improve HHcymediated angiogenic defects. PPAR-y is known to regulate angiogenesis via upregulation of VEGF levels (315), and VEGF can further activate eNOS during angiogenesis (320). Similar to the previous work this study showed that $\mathrm{H}_{2} \mathrm{~S}$ could 
induce PPAR-y expression (323), which suggest that $\mathrm{H}_{2} \mathrm{~S}$ may improve angiogenesis during chronic HHcy condition via PPAR-y/VEGF axis.

In chapter VI, I demonstrated the beneficial effect of $\mathrm{H}_{2} \mathrm{~S}$ in the mitigation of HHcy-mediated skeletal muscle atrophy via JNK/FOXO axis. I found a reduction of body weights in $\mathrm{CBS}^{+/-}$mice compared to age-matched WT mice and since their tibia lengths were similar, thus this effect may partly be due to the reduction of muscle mass. Then, to study whether this reduction of muscle mass can impair muscle function, I did whole animal muscle fatigability tests (by swim performance and grip strength test). The findings showed that HHcy induced muscle fatigability in $\mathrm{CBS}^{+/-}$mice compared to WT mice and it was noticed that NaHS supplementation for 8 weeks was able to improve this effect. I also demonstrated that HHcy induced fibrosis, collagen deposition and reduced CSAs of skeletal muscle fibers in the gastrocnemius muscle of $\mathrm{CBS}^{+/-}$mice compared to WT mice; interestingly NaHS supplementation improved this condition. I elucidated that actually HHcy induced muscle atrophy through upregulation of MuRF-1 and Atrogin-1 expressions via JNK/FOXO1 axis. Previous studies showed that Akt inhibits FOXO1 activity via preventing its nuclear localization, whereas JNK increases FOXO1 activity by promoting its import into the nucleus $(352,356-359)$. This study showed that Akt-phosphorylation was attenuated, and JNK-phosphorylation was induced after Hcy treatment in $\mathrm{C} 2 \mathrm{C} 12$ cells compared to vehicle controls, which possibly stabilizes FOXO1 in the nucleus and allows it to promote the expression of Atrogin-1 and MuRF-1 (E3 ubiquitin ligases). Whereas the upregulation of MuRF1 and Atrogin-1 due to HHcy condition may degrade their target proteins in the muscle fibers as reported previously for being responsible for muscle atrophy (361). Indeed, in this study, I noticed that upregulation of MuRF-1 degraded MHC-1 in $\mathrm{CBS}^{+/-}$mice compared to WT mice and this effect was substantially mitigated via NaHS treatment for 8 weeks.

The overall results from this study demonstrated that HHcy can cause skeletal 
muscle myopathy via cellular stress responses, apoptosis, inflammation, atrophy, and poor angiogenesis and identified a novel mechanism as to how $\mathrm{H}_{2} \mathrm{~S}$ could mitigate these effects.

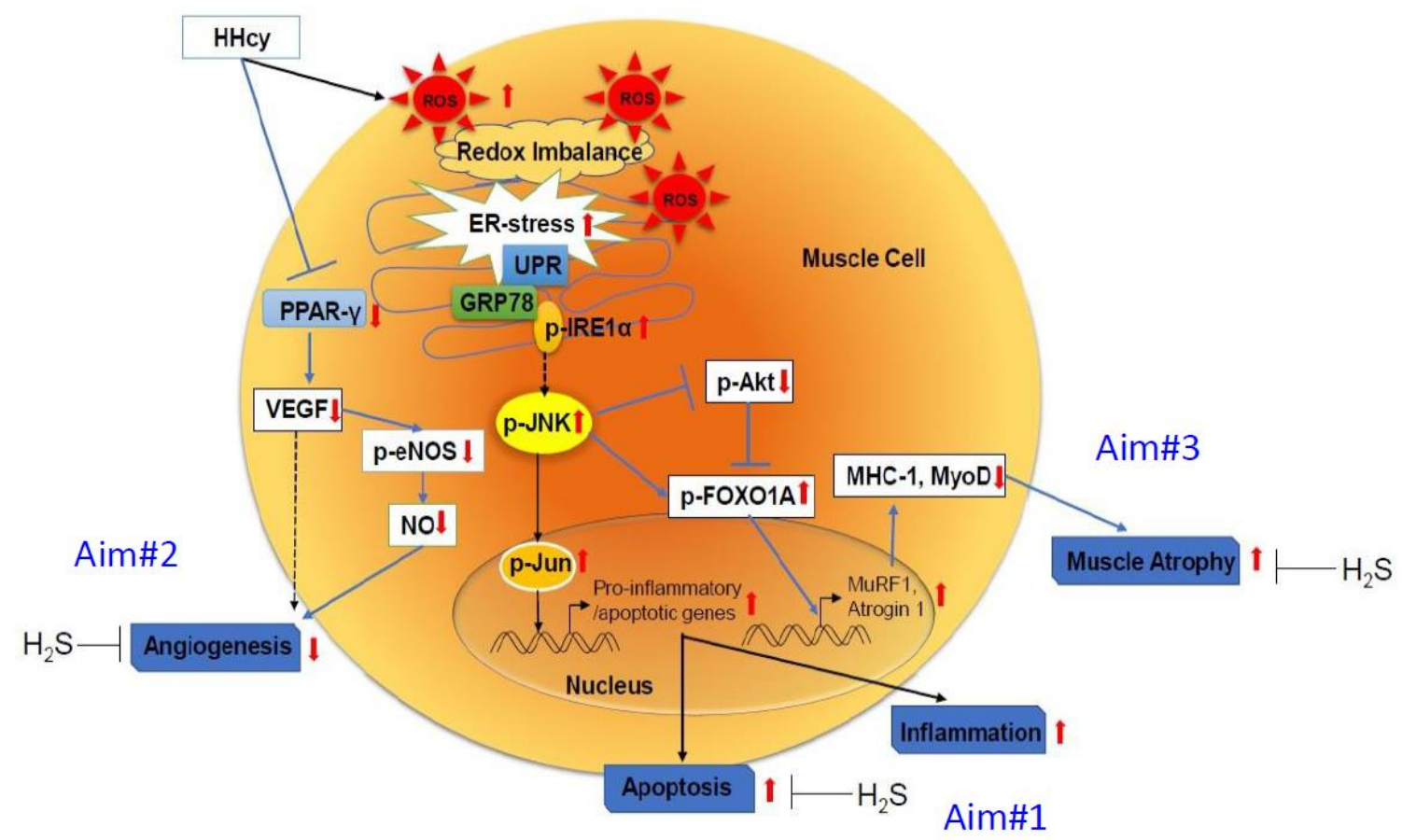

Figure 25. Proposed model. Based upon the above findings, I have proposed a model that I firmly believe to be involved in HHcy-mediated skeletal muscle dysfunction.

However, there is still a lot more left to clarify in this regard, such as (i) people develop HHcy due to many reasons; mutation of the $\mathrm{CBS}^{+-}$gene is one of them. I studied the effects of HHcy on skeletal muscle using $\mathrm{CBS}^{+/-}$mouse model that is similar to the physiological conditions but not identical as observed in HHcy patients, so a suitable animal model is still lacking. The half-life of NaHS is short, therefore most of the effects of $\mathrm{H}_{2} \mathrm{~S}$ vary in different studies. Moreover, delivery methods of $\mathrm{H}_{2} \mathrm{~S}$ donors (NaHS, Na2S, GYY4137, etc.) are also a crucial factor because of changes in the pharmacokinetics and pharmacodynamics of these compounds. Also, there is a lot of hurdles to overcome with regard to identifying a proper delivery method for $\mathrm{H}_{2} \mathrm{~S}$. Skeletal 
muscle dysfunction ranges from general muscle weakness and soreness to severe myopathy, muscle wasting and cachexia. Some of the complex dysfunctions do not necessarily originate from skeletal muscle and there are many variables and signaling pathways that need to be determined for devising the strategies to alleviate these pathological effects of HHcy on skeletal muscle myopathy. Although many studies have been devoted to clarifying the molecular mechanisms involved in E-C coupling (excitation-contraction coupling) during skeletal muscle atrophy this was not assessed in my study due to limited resources and complexity associated with testing under in-vivo settings. I analyzed the FOXO1A localization via ICC experiment, however, I didn't confirm my findings via western blot analysis using fractioned samples (nuclear and cytoplasmic extracts); (vi) Finally, from this study it is not still confirmed whether $\mathrm{H}_{2} \mathrm{~S}$ is working via oxidative stress mechanisms or something else and whether other antioxidants (like $\mathrm{N}$-acetylcysteine) has similar effects like $\mathrm{H}_{2} \mathrm{~S}$. So, future work should address these questions to devise potential therapeutic options.

In brief, this work provides evidence that $\mathrm{H}_{2} \mathrm{~S}$ is beneficial towards mitigating the HHcy-mediated skeletal injuries incited by oxidative/ER-stress responses. The findings from my study suggest that HHcy can inhibit angiogenesis via antagonizing the angiogenic signal pathways encompassing the PPAR- $\gamma$ /VEGF axis, and $\mathrm{H}_{2} \mathrm{~S}$ might be employed as a potential therapeutic option to alleviate the harmful metabolic effects of HHcy conditions. The findings from this study have been summarized in Fig. 23 highlighting the sequential events during $\mathrm{HHcy}$ and its effects on skeletal muscle atrophy. 


\section{FUTURE DIRECTIONS}

HHcy-mediated skeletal muscle dysfunction involves a great number of cytokines, DNA methylation, metabolic alterations, hormonal regulation, etc. (7). The primary focus of this study was to determine how HHcy promotes skeletal muscle myopathy through induction of oxidative and ER-stress responses, apoptosis, atrophy, and impairment of angiogenesis, and to study whether or not $\mathrm{H}_{2} \mathrm{~S}$ could mitigate the effects of HHcy during skeletal muscle myopathy. Although this study was able to demonstrate the above effects, there are many other factors and signaling pathways that need to be determined to devise any effective and potential treatment to improve these detrimental effects of HHcy in skeletal muscle dysfunction.

As I showed that NaHS improved skeletal muscle functions via mitigation of cellular stress responses using $\mathrm{CBS}^{+/-}$mouse model. However, people develop HHcy due to various other reasons. The western diet contains high levels of methionine (such as in red meat), and studies showed that an excessive intake of the high methionine diet could elevate Hcy levels $(79,246)$. So, to elaborate further, I wish to study and extend my hypothesis to in a diet-induced HHcy model.

As ER is considered as a storehouse of $\mathrm{Ca}^{2+}$ and alterations of $\mathrm{Ca}^{2+}$ release from ER contributes to dysregulation of muscle's contractility. In this study, I found HHcy mediated ER-stress in muscle, however, the effects of HHcy on sarcoplasmic reticulum's $\mathrm{Ca}^{2+}$ handling and whether that has any role in skeletal muscle fatigability remains unresolved. In the future, I want to study the effects of HHcy on E-C coupling, $\mathrm{Ca}^{2+}$ 
release, ATP/ADP ratios, mitochondrial biogenesis in the skeletal muscle.

It has been known that aging is associated with a decline in skeletal muscle mass and strength and a slowing of muscle contraction impact the quality of life for the older people. Previous studies demonstrated that older people have significantly higher levels of Hcy compared to younger ones $(387,388)$. It is also reported that HHcy can significantly lower physical functions in older people compared to age-matched healthy subjects (138), which suggests that aging is one of the potential risk factors to be considered in this process (139). In the future, I also want to study the role of Hcy in decline in physical function during the aging process.

Loss of skeletal muscle mass (i.e., muscle wasting) is a major component of cachexia; in particular, malnutrition is considered a critical factor $(389,390)$. Whereas plasma total Hcy levels are dependent on nutritional status (391), deficiency of folic acid and vitamins B6, B12 and B9 are also the causal factors for the induction of HHcy (391). Since the effect of Hcy for causation of cachexia is not studied. So, in the future, I want to examine my hypothesis in light of muscle cachexia. 


\section{REFERENCES}

1. Majumder A, Singh M, George AK, Tyagi SC. Restoration of skeletal muscle homeostasis by hydrogen sulfide during hyperhomocysteinemia-mediated oxidative/ER-stress condition. Canadian journal of physiology and pharmacology 2018;

2. Stipanuk MH, Ueki I. Dealing with methionine/homocysteine sulfur: cysteine metabolism to taurine and inorganic sulfur. Journal of inherited metabolic disease 2011; 34:17-32

3. Hankey GJ, Eikelboom JW. Homocysteine and vascular disease. Lancet (London, England) 1999; 354:407-413

4. Narayanan N, Pushpakumar SB, Givvimani S, Kundu S, Metreveli N, James D, Bratcher AP, Tyagi SC. Epigenetic regulation of aortic remodeling in hyperhomocysteinemia. FASEB journal : official publication of the Federation of American Societies for Experimental Biology 2014; 28:3411-3422

5. Veeranki S, Tyagi SC. Defective homocysteine metabolism: potential implications for skeletal muscle malfunction. International journal of molecular sciences 2013; 14:15074-15091

6. Cascella M, Arcamone M, Morelli E, Viscardi D, Russo V, De Franciscis S, Belli A, Accardo R, Caliendo D, De Luca E, Di Caprio B, Di Sauro F, Giannoni G, lermano C, Maciariello M, Marracino M, Cuomo A. Erratum to: Multidisciplinary approach and anesthetic management of a surgical cancer patient with methylene tetrahydrofolate reductase deficiency: a case report and review of the literature. Journal of medical case reports 2015; 9:218

7. Majumder A, Behera J, Jeremic N, Tyagi SC. Hypermethylation: Causes and Consequences in Skeletal Muscle Myopathy. Journal of cellular biochemistry 2017; 118:2108-2117

8. Lehotsky J, Tothova B, Kovalska M, Dobrota D, Benova A, Kalenska D, Kaplan P. Role of Homocysteine in the Ischemic Stroke and Development of Ischemic Tolerance. Frontiers in neuroscience 2016; 10:538

9. Haulrik N, Toubro S, Dyerberg J, Stender S, Skov AR, Astrup A. Effect of protein and methionine intakes on plasma homocysteine concentrations: a 6-mo randomized controlled trial in overweight subjects. The American journal of clinical nutrition 2002; 76:1202-1206

10. Al-Maskari MY, Waly MI, Ali A, Al-Shuaibi YS, Ouhtit A. Folate and vitamin B12 deficiency and hyperhomocysteinemia promote oxidative stress in adult type 2 diabetes. Nutrition (Burbank, Los Angeles County, Calif) 2012; 28:e23-26 
11. Jhee $\mathrm{KH}$, Kruger WD. The role of cystathionine beta-synthase in homocysteine metabolism. Antioxidants \& redox signaling 2005; 7:813-822

12. Sen U, Mishra PK, Tyagi N, Tyagi SC. Homocysteine to hydrogen sulfide or hypertension. Cell biochemistry and biophysics 2010; 57:49-58

13. Voskoboeva E, Semyachkina A, Yablonskaya M, Nikolaeva E. Homocystinuria due to cystathionine beta-synthase (CBS) deficiency in Russia: Molecular and clinical characterization. Molecular genetics and metabolism reports 2018; 14:47-54

14. Brustolin S, Giugliani R, Felix TM. Genetics of homocysteine metabolism and associated disorders. Brazilian journal of medical and biological research $=$ Revista brasileira de pesquisas medicas e biologicas 2010; 43:1-7

15. Kanwar YS, Manaligod JR, Wong PW. Morphologic studies in a patient with homocystinuria due to 5, 10-methylenetetrahydrofolate reductase deficiency. Pediatric research 1976; 10:598-609

16. Valentino F, Bivona G, Butera D, Paladino P, Fazzari M, Piccoli T, Ciaccio M, La Bella V. Elevated cerebrospinal fluid and plasma homocysteine levels in ALS. Eur J Neurol 2010; 17:84-89

17. Zoccolella S, Simone IL, Lamberti P, Samarelli V, Tortelli R, Serlenga L, Logroscino G. Elevated plasma homocysteine levels in patients with amyotrophic lateral sclerosis. Neurology 2008; 70:222-225

18. Kolling J, Scherer EB, Siebert C, Hansen F, Torres FV, Scaini G, Ferreira G, de Andrade RB, Goncalves CA, Streck EL, Wannmacher CM, Wyse AT. Homocysteine induces energy imbalance in rat skeletal muscle: is creatine a protector? Cell biochemistry and function 2013; 31:575-584

19. Miller A, Mujumdar V, Shek E, Guillot J, Angelo M, Palmer L, Tyagi SC. Hyperhomocyst(e)inemia induces multiorgan damage. Heart and vessels 2000; 15:135-143

20. Veeranki S, Winchester $\amalg$, Tyagi SC. Hyperhomocysteinemia associated skeletal muscle weakness involves mitochondrial dysfunction and epigenetic modifications. Biochimica et biophysica acta 2015; 1852:732-741

21. Kalra BR, Ghose S, Sood NN. Homocystinuria with bilateral absolute glaucoma. Indian journal of ophthalmology 1985; 33:195-197

22. Dupont-Versteegden EE. Apoptosis in muscle atrophy: relevance to sarcopenia. Experimental gerontology 2005; 40:473-481

23. Powers SK, Lynch GS, Murphy KT, Reid MB, Zijdewind I. Disease-Induced Skeletal Muscle Atrophy and Fatigue. Medicine and science in sports and exercise 2016; 48:2307-2319

24. Tyagi N, Sedoris KC, Steed M, Ovechkin AV, Moshal KS, Tyagi SC. Mechanisms of homocysteine-induced oxidative stress. American journal of physiology Heart and circulatory physiology 2005; 289:H2649-2656

25. Yan SK, Chang T, Wang H, Wu L, Wang R, Meng QH. Effects of hydrogen sulfide on homocysteine-induced oxidative stress in vascular smooth muscle cells. Biochemical and biophysical research communications 2006; 351:485-491

26. Matte C, Stefanello FM, Mackedanz V, Pederzolli CD, Lamers ML, Dutra-Filho CS, Dos Santos MF, Wyse AT. Homocysteine induces oxidative stress, inflammatory 
infiltration, fibrosis and reduces glycogen/glycoprotein content in liver of rats. International journal of developmental neuroscience : the official journal of the International Society for Developmental Neuroscience 2009; 27:337-344

27. George AK, Behera J, Kelly KE, Mondal NK, Richardson KP, Tyagi N. Exercise Mitigates Alcohol Induced Endoplasmic Reticulum Stress Mediated Cognitive Impairment through ATF6-Herp Signaling. Scientific reports 2018; 8:5158

28. Plaisance V, Brajkovic S, Tenenbaum M, Favre D, Ezanno $H$, Bonnefond A, Bonner C, Gmyr V, Kerr-Conte J, Gauthier BR, Widmann C, Waeber G, Pattou F, Froguel P, Abderrahmani A. Endoplasmic Reticulum Stress Links Oxidative Stress to Impaired Pancreatic Beta-Cell Function Caused by Human Oxidized LDL. PloS one 2016; 11:e0163046

29. Merad-Boudia M, Nicole A, Santiard-Baron D, Saille C, Ceballos-Picot I. Mitochondrial impairment as an early event in the process of apoptosis induced by glutathione depletion in neuronal cells: relevance to Parkinson's disease. Biochemical pharmacology 1998; 56:645-655

30. Nakamura T, Lipton SA. Molecular mechanisms of nitrosative stress-mediated protein misfolding in neurodegenerative diseases. Cellular and molecular life sciences : CMLS 2007; 64:1609-1620

31. Uehara T, Nakamura T, Yao D, Shi ZQ, Gu Z, Ma Y, Masliah E, Nomura Y, Lipton SA. S-nitrosylated protein-disulphide isomerase links protein misfolding to neurodegeneration. Nature 2006; 441:513-517

32. Zhang K, Kaufman RJ. From endoplasmic-reticulum stress to the inflammatory response. Nature 2008; 454:455-462

33. Werstuck GH, Lentz SR, Dayal S, Hossain GS, Sood SK, Shi YY, Zhou J, Maeda N, Krisans SK, Malinow MR, Austin RC. Homocysteine-induced endoplasmic reticulum stress causes dysregulation of the cholesterol and triglyceride biosynthetic pathways. The Journal of clinical investigation 2001; 107:1263-1273

34. Lei $\mathrm{K}$, Davis RJ. JNK phosphorylation of Bim-related members of the Bcl2 family induces Bax-dependent apoptosis. Proceedings of the National Academy of Sciences of the United States of America 2003; 100:2432-2437

35. Deng X, Xiao L, Lang W, Gao F, Ruvolo P, May WS, Jr. Novel role for JNK as a stressactivated Bcl2 kinase. The Journal of biological chemistry 2001; 276:23681-23688

36. Sano R, Reed JC. ER stress-induced cell death mechanisms. Biochimica et biophysica acta 2013; 1833:3460-3470

37. Manning BD, Cantley LC. AKT/PKB signaling: navigating downstream. Cell 2007; 129:1261-1274

38. Bodine SC, Stitt TN, Gonzalez M, Kline WO, Stover GL, Bauerlein R, Zlotchenko E, Scrimgeour A, Lawrence JC, Glass DJ, Yancopoulos GD. Akt/mTOR pathway is a crucial regulator of skeletal muscle hypertrophy and can prevent muscle atrophy in vivo. Nature cell biology 2001; 3:1014-1019

39. Romanino K, Mazelin L, Albert V, Conjard-Duplany A, Lin S, Bentzinger CF, Handschin C, Puigserver P, Zorzato F, Schaeffer L, Gangloff YG, Ruegg MA. Myopathy caused by mammalian target of rapamycin complex 1 (mTORC1) inactivation is not reversed by restoring mitochondrial function. Proceedings of 
the National Academy of Sciences of the United States of America 2011; 108:20808-20813

40. Aguirre V, Uchida T, Yenush L, Davis R, White MF. The c-Jun NH(2)-terminal kinase promotes insulin resistance during association with insulin receptor substrate-1 and phosphorylation of Ser(307). The Journal of biological chemistry 2000; 275:9047-9054

41. Aguirre V, Werner ED, Giraud J, Lee YH, Shoelson SE, White MF. Phosphorylation of Ser307 in insulin receptor substrate-1 blocks interactions with the insulin receptor and inhibits insulin action. The Journal of biological chemistry 2002; 277:1531-1537

42. Ogata M, Hino $S$, Saito A, Morikawa $K$, Kondo $S$, Kanemoto $S$, Murakami T, Taniguchi M, Tanii I, Yoshinaga K, Shiosaka S, Hammarback JA, Urano F, Imaizumi K. Autophagy is activated for cell survival after endoplasmic reticulum stress. Molecular and cellular biology 2006; 26:9220-9231

43. Xie CM, Chan WY, Yu S, Zhao J, Cheng CH. Bufalin induces autophagy-mediated cell death in human colon cancer cells through reactive oxygen species generation and JNK activation. Free radical biology \& medicine 2011; 51:1365-1375

44. Dhanasekaran DN, Reddy EP. JNK signaling in apoptosis. Oncogene 2008; 27:62456251

45. McCully KS. Homocysteine, vitamins, and vascular disease prevention. The American journal of clinical nutrition 2007; 86:1563s-1568s

46. Veeranki S, Givvimani S, Pushpakumar S, Tyagi SC. Hyperhomocysteinemia attenuates angiogenesis through reduction of HIF-1alpha and PGC-1alpha levels in muscle fibers during hindlimb ischemia. Am J Physiol Heart Circ Physiol 2014; 306:H1116-1127

47. Chen Y, Zhao S, Wang Y, Li Y, Bai L, Liu R, Fan J, Liu E. Homocysteine reduces protein S-nitrosylation in endothelium. International journal of molecular medicine 2014; 34:1277-1285

48. Yu Z, Wang AM, Adachi H, Katsuno M, Sobue G, Yue Z, Robins DM, Lieberman AP. Macroautophagy is regulated by the UPR-mediator CHOP and accentuates the phenotype of SBMA mice. PLoS genetics 2011; 7:e1002321

49. Clavel S, Siffroi-Fernandez S, Coldefy AS, Boulukos K, Pisani DF, Derijard B. Regulation of the intracellular localization of Foxo3a by stress-activated protein kinase signaling pathways in skeletal muscle cells. Molecular and cellular biology 2010; 30:470-480

50. Shimizu H, Langenbacher AD, Huang J, Wang K, Otto G, Geisler R, Wang Y, Chen $J N$. The Calcineurin-FoxO-MuRF1 signaling pathway regulates myofibril integrity in cardiomyocytes. eLife 2017; 6

51. Mironov A, Seregina T, Nagornykh M, Luhachack LG, Korolkova N, Lopes LE, Kotova V, Zavilgelsky G, Shakulov R, Shatalin K, Nudler E. Mechanism of H2S-mediated protection against oxidative stress in Escherichia coli. Proceedings of the National Academy of Sciences of the United States of America 2017; 114:6022-6027 
52. Kimura $\mathrm{Y}$, Goto $\mathrm{Y}$, Kimura H. Hydrogen sulfide increases glutathione production and suppresses oxidative stress in mitochondria. Antioxidants \& redox signaling 2010; 12:1-13

53. Yang G, An SS, Ji Y, Zhang W, Pei Y. Hydrogen Sulfide Signaling in Oxidative Stress and Aging Development. Oxidative medicine and cellular longevity 2015; 2015:357824

54. Szabo C. Hydrogen sulphide and its therapeutic potential. Nature reviews Drug discovery 2007; 6:917-935

55. Wang R. The gasotransmitter role of hydrogen sulfide. Antioxidants \& redox signaling 2003; 5:493-501

56. Fiorucci S, Distrutti E, Cirino G, Wallace JL. The emerging roles of hydrogen sulfide in the gastrointestinal tract and liver. Gastroenterology 2006; 131:259-271

57. Calvert JW, Coetzee WA, Lefer DJ. Novel insights into hydrogen sulfide--mediated cytoprotection. Antioxidants \& redox signaling 2010; 12:1203-1217

58. Elsey DJ, Fowkes RC, Baxter GF. Regulation of cardiovascular cell function by hydrogen sulfide (H(2)S). Cell biochemistry and function 2010; 28:95-106

59. Gadalla MM, Snyder SH. Hydrogen sulfide as a gasotransmitter. Journal of neurochemistry 2010; 113:14-26

60. Kimura H. Hydrogen sulfide: from brain to gut. Antioxidants \& redox signaling 2010; 12:1111-1123

61. Predmore BL, Lefer DJ. Development of hydrogen sulfide-based therapeutics for cardiovascular disease. Journal of cardiovascular translational research 2010; 3:487-498

62. Kabil $O$, Vitvitsky $V$, Xie $P$, Banerjee $R$. The quantitative significance of the transsulfuration enzymes for $\mathrm{H} 2 \mathrm{~S}$ production in murine tissues. Antioxidants \& redox signaling 2011; 15:363-372

63. Beard RS, Jr., Bearden SE. Vascular complications of cystathionine beta-synthase deficiency: future directions for homocysteine-to-hydrogen sulfide research. American journal of physiology Heart and circulatory physiology 2011; 300:H1326

64. Nandi SS, Mishra PK. H2S and homocysteine control a novel feedback regulation of cystathionine beta synthase and cystathionine gamma lyase in cardiomyocytes. Scientific reports 2017; 7:3639

65. Yang A, Jiao Y, Yang S, Deng M, Yang X, Mao C, Sun Y, Ding N, Li N, Zhang M, Jin S, Zhang $H$, Jiang $Y$. Homocysteine activates autophagy by inhibition of CFTR expression via interaction between DNA methylation and H3K27me3 in mouse liver. Cell death \& disease 2018; 9:169

66. Winchester LJ, Veeranki S, Pushpakumar S, Tyagi SC. Exercise mitigates the effects of hyperhomocysteinemia on adverse muscle remodeling. Physiological reports 2018; 6:e13637

67. Tyagi N, Qipshidze N, Sen U, Rodriguez W, Ovechkin A, Tyagi SC. Cystathionine beta synthase gene dose dependent vascular remodeling in murine model of hyperhomocysteinemia. International journal of physiology, pathophysiology and pharmacology 2011; 3:210-222 
68. Narayanan N, Tyagi N, Shah A, Pagni S, Tyagi SC. Hyperhomocysteinemia during aortic aneurysm, a plausible role of epigenetics. International journal of physiology, pathophysiology and pharmacology 2013; 5:32-42

69. Tyagi N, Qipshidze N, Munjal C, Vacek JC, Metreveli N, Givvimani S, Tyagi SC. Tetrahydrocurcumin ameliorates homocysteinylated cytochrome-c mediated autophagy in hyperhomocysteinemia mice after cerebral ischemia. Journal of molecular neuroscience : MN 2012; 47:128-138

70. Familtseva A, Kalani A, Chaturvedi P, Tyagi N, Metreveli N, Tyagi SC. Mitochondrial mitophagy in mesenteric artery remodeling in hyperhomocysteinemia. Physiological reports 2014; 2:e00283

71. Watanabe M, Osada J, Aratani Y, Kluckman K, Reddick R, Malinow MR, Maeda N. Mice deficient in cystathionine beta-synthase: animal models for mild and severe homocyst(e)inemia. Proceedings of the National Academy of Sciences of the United States of America 1995; 92:1585-1589

72. John A, Kundu S, Pushpakumar S, Fordham M, Weber G, Mukhopadhyay M, Sen U. GYY4137, a Hydrogen Sulfide Donor Modulates miR194-Dependent Collagen Realignment in Diabetic Kidney. Scientific reports 2017; 7:10924

73. Ishihara $H$, Kubota $H$, Lindberg RL, Leppert D, Gloor SM, Errede M, Virgintino D, Fontana A, Yonekawa Y, Frei K. Endothelial cell barrier impairment induced by glioblastomas and transforming growth factor beta2 involves matrix metalloproteinases and tight junction proteins. Journal of neuropathology and experimental neurology 2008; 67:435-448

74. Lan X, Field MS, Stover PJ. Cell cycle regulation of folate-mediated one-carbon metabolism. Wiley interdisciplinary reviews Systems biology and medicine 2018:e1426

75. Tiahou G, Dupuy AM, Jaussent I, Sees D, Cristol JP, Badiou S. Determinants of homocysteine levels in Ivorian rural population. International journal for vitamin and nutrition research Internationale Zeitschrift fur Vitamin- und Ernahrungsforschung Journal international de vitaminologie et de nutrition 2009; 79:319-327

76. Verhoef $P$, van Vliet $T$, Olthof $M R$, Katan MB. A high-protein diet increases postprandial but not fasting plasma total homocysteine concentrations: a dietary controlled, crossover trial in healthy volunteers. The American journal of clinical nutrition 2005; 82:553-558

77. Kharb S, Aggarwal D, Bala J, Nanda S. Evaluation of Homocysteine, Vitamin B12 and Folic Acid Levels During all the Trimesters in Pregnant and Preeclamptic Womens. Current hypertension reviews 2016; 12:234-238

78. Iqbal MP, Lindblad BS, Mehboobali N, Yusuf FA, Khan AH, Iqbal SP. Folic acid and vitamin B6 deficiencies related hyperhomocysteinemia in apparently healthy Pakistani adults; is mass micronutrient supplementation indicated in this population? Journal of the College of Physicians and Surgeons--Pakistan : JCPSP 2009; 19:308-312

79. Diakoumopoulou E, Tentolouris N, Kirlaki E, Perrea D, Kitsou E, Psallas M, Doulgerakis D, Katsilambros N. Plasma homocysteine levels in patients with type 
2 diabetes in a Mediterranean population: relation with nutritional and other factors. Nutrition, metabolism, and cardiovascular diseases : NMCD 2005; 15:109117

80. Maddocks OD, Labuschagne CF, Adams PD, Vousden KH. Serine Metabolism Supports the Methionine Cycle and DNA/RNA Methylation through De Novo ATP Synthesis in Cancer Cells. Molecular cell 2016; 61:210-221

81. Laha A, Majumdar A, Singh M, Tyagi SC. Connecting homocysteine and obesity through pyroptosis, gut microbiome, epigenetics, peroxisome proliferatoractivated receptor gamma, and zinc finger protein 407. Canadian journal of physiology and pharmacology 2018:1-6

82. Veeranki S, Tyagi SC. Mechanisms of hyperhomocysteinemia induced skeletal muscle myopathy after ischemia in the CBS-/+ mouse model. International journal of molecular sciences 2015; 16:1252-1265

83. Winchester L, Veeranki S, Givvimani S, Tyagi SC. Exercise mitigates the adverse effects of hyperhomocysteinemia on macrophages, MMP-9, skeletal muscle, and white adipocytes. Canadian journal of physiology and pharmacology 2014; 92:575-582

84. Ishii I, Akahoshi N, Yamada H, Nakano S, Izumi T, Suematsu M. Cystathionine gamma-Lyase-deficient mice require dietary cysteine to protect against acute lethal myopathy and oxidative injury. The Journal of biological chemistry 2010; 285:26358-26368

85. Orendac M, Muskova B, Richterova E, Zvarova J, Stefek M, Zaykova E, Kraus JP, Stribrny J, Hyanek J, Kozich V. Is the common 844ins68 polymorphism in the cystathionine beta-synthase gene associated with atherosclerosis? Journal of inherited metabolic disease 1999; 22:674-675

86. Ding $R$, Lin S, Chen D. The association of cystathionine beta synthase (CBS) T833C polymorphism and the risk of stroke: a meta-analysis. Journal of the neurological sciences 2012; 312:26-30

87. de Franchis R, Fermo I, Mazzola G, Sebastio G, Di Minno G, Coppola A, Andria G, D'Angelo $A$. Contribution of the cystathionine beta-synthase gene (844ins68) polymorphism to the risk of early-onset venous and arterial occlusive disease and of fasting hyperhomocysteinemia. Thrombosis and haemostasis 2000; 84:576-582

88. McGimpsey SJ, Woodside JV, Cardwell C, Cahill M, Chakravarthy U. Homocysteine, methylenetetrahydrofolate reductase $\mathrm{C677T}$ polymorphism, and risk of retinal vein occlusion: a meta-analysis. Ophthalmology 2009; 116:1778-1787.e1771

89. Banerjee I, Gupta V, Ganesh S. Association of gene polymorphism with genetic susceptibility to stroke in Asian populations: a meta-analysis. Journal of human genetics 2007; 52:205-219

90. Gao S, Li H, Xiao H, Yao G, Shi Y, Wang Y, Zhou X, Yu H. Association of MTHFR 677T variant allele with risk of intracerebral haemorrhage: a meta-analysis. Journal of the neurological sciences 2012; 323:40-45

91. Kang S, Zhao X, Liu L, Wu W, Zhang D. Association of the C677T polymorphism in the MTHFR gene with hemorrhagic stroke: a meta-analysis. Genetic testing and molecular biomarkers 2013; 17:412-417 
92. $\mathrm{Yu} H \mathrm{H}$, Zhang $\mathrm{WL}$, Shi JP. [Relationship between methylenetetrahydrofolate reductase gene C677T polymorphism and susceptibility of ischemic stroke: a meta-analysis]. Zhonghua yi xue za zhi 2011; 91:2060-2064

93. Ray JG, Shmorgun D, Chan WS. Common C677T polymorphism of the methylenetetrahydrofolate reductase gene and the risk of venous thromboembolism: meta-analysis of 31 studies. Pathophysiology of haemostasis and thrombosis 2002; 32:51-58

94. Cai W, Yin L, Yang F, Zhang L, Cheng J. Association between Hcy levels and the CBS844ins68 and MTHFR C677T polymorphisms with essential hypertension. Biomedical reports 2014; 2:861-868

95. Heifetz EM, Birk RZ. MTHFR C677T polymorphism affects normotensive diastolic blood pressure independently of blood lipids. American journal of hypertension 2015; 28:387-392

96. Yang KM, Jia J, Mao LN, Men C, Tang KT, Li YY, Ding HX, Zhan YY. Methylenetetrahydrofolate reductase C677T gene polymorphism and essential hypertension: A meta-analysis of 10,415 subjects. Biomedical reports 2014; 2:699708

97. Hua $\mathrm{Y}$, Zhao $\mathrm{H}$, Kong $\mathrm{Y}, \mathrm{Ye} \mathrm{M}$. Association between the MTHFR gene and Alzheimer's disease: a meta-analysis. The International journal of neuroscience 2011; 121:462-471

98. Ibrahim S, Maqbool S, Azam M, lqbal MP, Qamar R. CBS mutations and MTFHR SNPs causative of hyperhomocysteinemia in Pakistani children. Molecular biology reports 2018; 45:353-360

99. Castro R, Rivera I, Blom HJ, Jakobs C, Tavares de Almeida I. Homocysteine metabolism, hyperhomocysteinaemia and vascular disease: an overview. Journal of inherited metabolic disease 2006; 29:3-20

100. White $\mathrm{RH}$. The epidemiology of venous thromboembolism. Circulation 2003; 107:14-8

101. Liang R, Zhou Y, Xie J, Lv W, Kang B, Liang Y, Chen Y, Li Y. [Association of C677T gene polymorphisms of methylenetetrahydrofolate reductase and plasma homocysteine level with hyperlipidemia]. Nan fang yi ke da xue xue bao = Journal of Southern Medical University 2014; 34:1195-1198

102. Al-Rubeaan K, Siddiqui K, Saeb AT, Nazir N, Al-Naqeb D, Al-Qasim S. ACE I/D and MTHFR C677T polymorphisms are significantly associated with type 2 diabetes in Arab ethnicity: a meta-analysis. Gene 2013; 520:166-177

103. Chang WW, Zhang L, Yao YS, Su H, Jin YL, Chen Y. Methylenetetrahydrofolate reductase (MTHFR) C677T polymorphism and susceptibility to diabetic nephropathy in Chinese type 2 diabetic patients: a meta-analysis. Renal failure 2013; 35:1038-1043

104. Yang S, Zhang J, Feng C, Huang G. MTHFR 677T variant contributes to diabetic nephropathy risk in Caucasian individuals with type 2 diabetes: a meta-analysis. Metabolism: clinical and experimental 2013; 62:586-594 
105. Zhang D, Zhou Y, Han L, Ji H, Li J. The effect of MTHFR C677T polymorphism on type 2 diabetes mellitus with vascular complications in Chinese Han population: a meta-analysis. Endocrine journal 2014; 61:717-726

106. Gouveia LO, Canhao P. MTHFR and the risk for cerebral venous thrombosis--a meta-analysis. Thrombosis research 2010; 125:e153-158

107. Wu CY, Yang M, Lin M, Li LP, Wen XZ. MTHFR C677T polymorphism was an ethnicity-dependent risk factor for cervical cancer development: evidence based on a meta-analysis. Archives of gynecology and obstetrics 2013; 288:595-605

108. Wu YL, Ding XX, Sun YH, Yang HY, Sun L. Methylenetetrahydrofolate reductase (MTHFR) C677T/A1298C polymorphisms and susceptibility to Parkinson's disease: a meta-analysis. Journal of the neurological sciences 2013; 335:14-21

109. Laster L, Mudd SH, Finkelstein JD, Irreverre F. Homocystinuria due to cystathionine synthase deficiency: the metabolism of L-methionine. The Journal of clinical investigation 1965; 44:1708-1719

110. Finkelstein JD, Martin JJ. Methionine metabolism in mammals. Distribution of homocysteine between competing pathways. The Journal of biological chemistry 1984; 259:9508-9513

111. Finkelstein JD, Kyle WE, Martin JL, Pick AM. Activation of cystathionine synthase by adenosylmethionine and adenosylethionine. Biochemical and biophysical research communications $1975 ; 66: 81-87$

112. Stabler SP, Steegborn C, Wahl MC, Oliveriusova J, Kraus JP, Allen RH, Wagner C, Mudd SH. Elevated plasma total homocysteine in severe methionine adenosyltransferase I/III deficiency. Metabolism: clinical and experimental 2002; 51:981-988

113. Mudd SH, Finkelstein JD, Irreverre F, Laster L. Transsulfuration in mammals. Microassays and tissue distributions of three enzymes of the pathway. The Journal of biological chemistry 1965; 240:4382-4392

114. Finkelstein JD. Methionine metabolism in mammals. The Journal of nutritional biochemistry 1990; 1:228-237

115. Stolzenberg-Solomon RZ, Miller ER, 3rd, Maguire MG, Selhub J, Appel U. Association of dietary protein intake and coffee consumption with serum homocysteine concentrations in an older population. The American journal of clinical nutrition 1999; 69:467-475

116. Selhub J. Homocysteine metabolism. Annual review of nutrition 1999; 19:217-246

117. Selhub J, Jacques PF, Rosenberg IH, Rogers G, Bowman BA, Gunter EW, Wright JD, Johnson CL. Serum total homocysteine concentrations in the third National Health and Nutrition Examination Survey (1991-1994): population reference ranges and contribution of vitamin status to high serum concentrations. Annals of internal medicine 1999; 131:331-339

118. Eloranta TO, Martikainen V, Smith TK. Adaptation of adenosylmethionine metabolism and methionine recycling to variations in dietary methionine in the rat. Proceedings of the Society for Experimental Biology and Medicine Society for Experimental Biology and Medicine (New York, NY) 1990; 194:364-371 
119. Gupta A, Moustapha A, Jacobsen DW, Goormastic M, Tuzcu EM, Hobbs R, Young J, James K, McCarthy P, van Lente F, Green R, Robinson K. High homocysteine, low folate, and low vitamin B6 concentrations: prevalent risk factors for vascular disease in heart transplant recipients. Transplantation 1998; 65:544-550

120. Pedersen BK. Muscle as a secretory organ. Comprehensive Physiology 2013; 3:1337-1362

121. Frontera WR, Ochala J. Skeletal muscle: a brief review of structure and function. Calcified tissue international 2015; 96:183-195

122. Smith IC, Bombardier E, Vigna C, Tupling AR. ATP consumption by sarcoplasmic reticulum $\mathrm{Ca}(2)(+)$ pumps accounts for $40-50 \%$ of resting metabolic rate in mouse fast and slow twitch skeletal muscle. PloS one 2013; 8:e68924

123. Talbot J, Maves L. Skeletal muscle fiber type: using insights from muscle developmental biology to dissect targets for susceptibility and resistance to muscle disease. Wiley interdisciplinary reviews Developmental biology 2016; 5:518-534

124. Allen DG, Westerblad $\mathrm{H}$. Understanding muscle from its length. The Journal of physiology 2007; 583:3-4

125. Mi-Mi L, Pruyne D. Loss of Sarcomere-associated Formins Disrupts Z-line Organization, but does not Prevent Thin Filament Assembly in Caenorhabditis elegans Muscle. Journal of cytology \& histology 2015; 6

126. Gehlert S, Bloch W, Suhr F. Ca2+-dependent regulations and signaling in skeletal muscle: from electro-mechanical coupling to adaptation. International journal of molecular sciences 2015; 16:1066-1095

127. Deschenes MR. Motor unit and neuromuscular junction remodeling with aging. Current aging science $2011 ; 4: 209-220$

128. Kuo IY, Ehrlich BE. Signaling in muscle contraction. Cold Spring Harbor perspectives in biology 2015; 7:a006023

129. Mauro A. Satellite cell of skeletal muscle fibers. The Journal of biophysical and biochemical cytology 1961; 9:493-495

130. Mitchell PO, Pavlath GK. A muscle precursor cell-dependent pathway contributes to muscle growth after atrophy. American journal of physiology Cell physiology 2001; 281:C1706-1715

131. Rosenblatt JD, Yong D, Parry DJ. Satellite cell activity is required for hypertrophy of overloaded adult rat muscle. Muscle \& nerve 1994; 17:608-613

132. Charge SB, Rudnicki MA. Cellular and molecular regulation of muscle regeneration. Physiological reviews 2004; 84:209-238

133. Hawke TJ, Garry DJ. Myogenic satellite cells: physiology to molecular biology. Journal of applied physiology (Bethesda, Md : 1985) 2001; 91:534-551

134. Miyake M, Hayashi S, Iwasaki S, Uchida T, Watanabe K, Ohwada S, Aso H, Yamaguchi T. TIEG1 negatively controls the myoblast pool indispensable for fusion during myogenic differentiation of C2C12 cells. Journal of cellular physiology 2011; 226:1128-1136

135. Tidball JG, Albrecht DE, Lokensgard BE, Spencer MJ. Apoptosis precedes necrosis of dystrophin-deficient muscle. Journal of cell science 1995; 108 ( Pt 6):2197-2204 
136. Tews DS, Goebel HH. DNA-fragmentation and expression of apoptosis-related proteins in muscular dystrophies. Neuropathology and applied neurobiology 1997; 23:331-338

137. Ganguly P, Alam SF. Role of homocysteine in the development of cardiovascular disease. Nutrition journal 2015; 14:6

138. Kado DM, Bucur A, Selhub J, Rowe JW, Seeman T. Homocysteine levels and decline in physical function: MacArthur Studies of Successful Aging. The American journal of medicine 2002; 113:537-542

139. Westerblad H, Place N, Yamada T. Mechanisms of skeletal muscle weakness. Advances in experimental medicine and biology 2010; 682:279-296

140. Swart KM, van Schoor NM, Heymans MW, Schaap LA, den Heijer M, Lips P. Elevated homocysteine levels are associated with low muscle strength and functional limitations in older persons. The journal of nutrition, health \& aging 2013; 17:578-584

141. Yamamoto M, Yang G, Hong C, Liu J, Holle E, Yu X, Wagner T, Vatner SF, Sadoshima J. Inhibition of endogenous thioredoxin in the heart increases oxidative stress and cardiac hypertrophy. The Journal of clinical investigation 2003; 112:1395-1406

142. Zima AV, Copello JA, Blatter LA. Effects of cytosolic NADH/NAD(+) levels on sarcoplasmic reticulum $\mathrm{Ca}(2+)$ release in permeabilized rat ventricular myocytes. The Journal of physiology 2004; 555:727-741

143. Gasparetto C, Malinverno A, Culacciati D, Gritti D, Prosperini PG, Specchia G, Ricevuti G. Antioxidant vitamins reduce oxidative stress and ventricular remodeling in patients with acute myocardial infarction. International journal of immunopathology and pharmacology 2005; 18:487-496

144. Lubos $E$, Handy DE, Loscalzo J. Role of oxidative stress and nitric oxide in atherothrombosis. Frontiers in bioscience : a journal and virtual library 2008; 13:5323-5344

145. Lentz SR. Mechanisms of homocysteine-induced atherothrombosis. J Thromb Haemost 2005; 3:1646-1654

146. Hayden MR, Tyagi SC. Homocysteine and reactive oxygen species in metabolic syndrome, type 2 diabetes mellitus, and atheroscleropathy: the pleiotropic effects of folate supplementation. Nutrition journal 2004; 3:4

147. Pacifici RE, Davies KJ. Protein, lipid and DNA repair systems in oxidative stress: the free-radical theory of aging revisited. Gerontology 1991; 37:166-180

148. Mishra PK, Tyagi N, Sen U, Joshua IG, Tyagi SC. Synergism in hyperhomocysteinemia and diabetes: role of PPAR gamma and tempol. Cardiovascular diabetology 2010; 9:49

149. Malhotra JD, Miao H, Zhang K, Wolfson A, Pennathur S, Pipe SW, Kaufman RJ. Antioxidants reduce endoplasmic reticulum stress and improve protein secretion. Proceedings of the National Academy of Sciences of the United States of America 2008; 105:18525-18530

150. Kokame K, Kato H, Miyata T. Homocysteine-respondent genes in vascular endothelial cells identified by differential display analysis. GRP78/BiP and novel genes. The Journal of biological chemistry 1996; 271:29659-29665 
151. Panayi GS, Corrigall VM. Immunoglobulin heavy-chain-binding protein (BiP): a stress protein that has the potential to be a novel therapy for rheumatoid arthritis. Biochemical Society transactions 2014; 42:1752-1755

152. Oslowski CM, Urano F. Measuring ER stress and the unfolded protein response using mammalian tissue culture system. Methods in enzymology 2011; 490:71-92

153. Kaufman RJ. Orchestrating the unfolded protein response in health and disease. The Journal of clinical investigation 2002; 110:1389-1398

154. Mori K. Tripartite management of unfolded proteins in the endoplasmic reticulum. Cell 2000; 101:451-454

155. Ron D. Translational control in the endoplasmic reticulum stress response. The Journal of clinical investigation 2002; 110:1383-1388

156. Rutkowski DT, Kaufman RJ. A trip to the ER: coping with stress. Trends in cell biology 2004; 14:20-28

157. Adham IM, Eck TJ, Mierau K, Muller N, Sallam MA, Paprotta I, Schubert S, HoyerFender S, Engel W. Reduction of spermatogenesis but not fertility in Creb3/4deficient mice. Molecular and cellular biology 2005; 25:7657-7664

158. Kondo S, Murakami T, Tatsumi K, Ogata M, Kanemoto S, Otori K, Iseki K, Wanaka A, Imaizumi K. OASIS, a CREB/ATF-family member, modulates UPR signalling in astrocytes. Nature cell biology 2005; 7:186-194

159. Zhang K, Shen X, Wu J, Sakaki K, Saunders T, Rutkowski DT, Back SH, Kaufman RJ. Endoplasmic reticulum stress activates cleavage of CREBH to induce a systemic inflammatory response. Cell 2006; 124:587-599

160. Cao SS. Endoplasmic reticulum stress and unfolded protein response in inflammatory bowel disease. Inflammatory bowel diseases 2015; 21:636-644

161. Shamu CE, Walter P. Oligomerization and phosphorylation of the Ire1p kinase during intracellular signaling from the endoplasmic reticulum to the nucleus. The EMBO journal 1996; 15:3028-3039

162. Hassler J, Cao SS, Kaufman RJ. IRE1, a double-edged sword in pre-miRNA slicing and cell death. Developmental cell 2012; 23:921-923

163. Gardner BM, Pincus D, Gotthardt K, Gallagher CM, Walter P. Endoplasmic reticulum stress sensing in the unfolded protein response. Cold Spring Harbor perspectives in biology 2013; 5:a013169

164. Hollien J, Lin JH, Li H, Stevens N, Walter P, Weissman JS. Regulated Ire1-dependent decay of messenger RNAs in mammalian cells. The Journal of cell biology 2009; 186:323-331

165. Maurel M, Chevet E, Tavernier J, Gerlo S. Getting RIDD of RNA: IRE1 in cell fate regulation. Trends in biochemical sciences 2014; 39:245-254

166. Rasool RU, Nayak D, Chakraborty S, Faheem MM, Rah B, Mahajan P, Gopinath V, Katoch A, Iqra Z, Yousuf SK, Mukherjee D, Kumar LD, Nargotra A, Goswami A. AKT is indispensable for coordinating Par-4/JNK cross talk in p21 downmodulation during ER stress. Oncogenesis 2017; 6:e341

167. Park GB, Jeong JY, Kim D. Ampelopsin-induced reactive oxygen species enhance the apoptosis of colon cancer cells by activating endoplasmic reticulum stressmediated AMPK/MAPK/XAF1 signaling. Oncology letters 2017; 14:7947-7956 
168. Darling NJ, Cook SJ. The role of MAPK signalling pathways in the response to endoplasmic reticulum stress. Biochimica et biophysica acta 2014; 1843:21502163

169. Renehan AG, Booth C, Potten CS. What is apoptosis, and why is it important? BMJ (Clinical research ed) 2001; 322:1536-1538

170. Thompson CB. Apoptosis in the pathogenesis and treatment of disease. Science (New York, NY) 1995; 267:1456-1462

171. Kerr JF, Winterford CM, Harmon BV. Apoptosis. Its significance in cancer and cancer therapy. Cancer 1994; 73:2013-2026

172. Lockshin RA, Zakeri Z. Cell death in health and disease. Journal of cellular and molecular medicine 2007; 11:1214-1224

173. Elmore S. Apoptosis: a review of programmed cell death. Toxicologic pathology 2007; 35:495-516

174. Zamzami N, Susin SA, Marchetti P, Hirsch T, Gomez-Monterrey I, Castedo M, Kroemer G. Mitochondrial control of nuclear apoptosis. The Journal of experimental medicine 1996; 183:1533-1544

175. Green DR, Reed JC. Mitochondria and apoptosis. Science (New York, NY) 1998; 281:1309-1312

176. Morel Y, Barouki R. Repression of gene expression by oxidative stress. The Biochemical journal 1999; 342 Pt 3:481-496

177. Richter C. Pro-oxidants and mitochondrial $\mathrm{Ca} 2+$ : their relationship to apoptosis and oncogenesis. FEBS letters 1993; 325:104-107

178. Okamoto A, Tanaka M, Sumi C, Oku K, Kusunoki M, Nishi K, Matsuo Y, Takenaga K, Shingu K, Hirota K. The antioxidant N-acetyl cysteine suppresses lidocaine-induced intracellular reactive oxygen species production and cell death in neuronal $\mathrm{SH}$ SY5Y cells. BMC anesthesiology 2016; 16:104

179. Kannan K, Jain SK. Oxidative stress and apoptosis. Pathophysiology : the official journal of the International Society for Pathophysiology 2000; 7:153-163

180. Bessede G, Miguet C, Gambert P, Neel D, Lizard G. Efficiency of homocysteine plus copper in inducing apoptosis is inversely proportional to gamma-glutamyl transpeptidase activity. FASEB journal : official publication of the Federation of American Societies for Experimental Biology 2001; 15:1927-1940

181. Sipkens JA, Hahn N, van den Brand CS, Meischl C, Cillessen SA, Smith DE, Juffermans LJ, Musters RJ, Roos D, Jakobs C, Blom HJ, Smulders YM, Krijnen PA, Stehouwer CD, Rauwerda JA, van Hinsbergh VW, Niessen HW. Homocysteineinduced apoptosis in endothelial cells coincides with nuclear NOX2 and perinuclear NOX4 activity. Cell biochemistry and biophysics 2013; 67:341-352

182. Zhang C, Cai Y, Adachi MT, Oshiro S, Aso T, Kaufman RJ, Kitajima S. Homocysteine induces programmed cell death in human vascular endothelial cells through activation of the unfolded protein response. The Journal of biological chemistry 2001; 276:35867-35874

183. Di Simone N, Maggiano N, Caliandro D, Riccardi P, Evangelista A, Carducci B, Caruso A. Homocysteine induces trophoblast cell death with apoptotic features. Biology of reproduction 2003; 69:1129-1134 
184. Cai B, Li X, Wang Y, Liu Y, Yang F, Chen H, Yin K, Tan X, Zhu J, Pan Z, Wang B, Lu Y. Apoptosis of bone marrow mesenchymal stem cells caused by homocysteine via activating JNK signal. PloS one 2013; 8:e63561

185. Hsu HC, Chiou JF, Wang YH, Chen $\mathrm{CH}$, Mau SY, Ho CT, Chang PJ, Liu TZ, Chen CH. Folate deficiency triggers an oxidative-nitrosative stress-mediated apoptotic cell death and impedes insulin biosynthesis in RINm5F pancreatic islet beta-cells: relevant to the pathogenesis of diabetes. PloS one 2013; 8:e77931

186. Kang SS, Wong PW, Norusis M. Homocysteinemia due to folate deficiency. Metabolism: clinical and experimental 1987; 36:458-462

187. Shastry S, Ingram AJ, Scholey JW, James LR. Homocysteine induces mesangial cell apoptosis via activation of p38-mitogen-activated protein kinase. Kidney international 2007; 71:304-311

188. Kruman, II, Culmsee C, Chan SL, Kruman Y, Guo Z, Penix L, Mattson MP. Homocysteine elicits a DNA damage response in neurons that promotes apoptosis and hypersensitivity to excitotoxicity. The Journal of neuroscience : the official journal of the Society for Neuroscience 2000; 20:6920-6926

189. Familtseva A, Chaturvedi P, Kalani A, Jeremic N, Metreveli N, Kunkel GH, Tyagi SC. Toll-like receptor 4 mutation suppresses hyperhomocysteinemia-induced hypertension. American journal of physiology Cell physiology 2016; 311:C596c606

190. Tang $X Q$, Chen RQ, Ren YK, Soldato PD, Sparatore A, Zhuang YY, Fang HR, Wang CY. ACS6, a Hydrogen sulfide-donating derivative of sildenafil, inhibits homocysteine-induced apoptosis by preservation of mitochondrial function. Medical gas research 2011; 1:20

191. Olfert IM, Baum O, Hellsten Y, Egginton S. Advances and challenges in skeletal muscle angiogenesis. American journal of physiology Heart and circulatory physiology 2016; 310:H326-336

192. Yan Z, Okutsu M, Akhtar YN, Lira VA. Regulation of exercise-induced fiber type transformation, mitochondrial biogenesis, and angiogenesis in skeletal muscle. Journal of applied physiology (Bethesda, Md : 1985) 2011; 110:264-274

193. Evans WJ, Paolisso G, Abbatecola AM, Corsonello A, Bustacchini S, Strollo F, Lattanzio F. Frailty and muscle metabolism dysregulation in the elderly. Biogerontology 2010; 11:527-536

194. Refsum H, Ueland PM, Nygard O, Vollset SE. Homocysteine and cardiovascular disease. Annual review of medicine 1998; 49:31-62

195. Loscalzo J. Homocysteine-mediated thrombosis and angiostasis in vascular pathobiology. The Journal of clinical investigation 2009; 119:3203-3205

196. Logsdon EA, Finley SD, Popel AS, Mac Gabhann F. A systems biology view of blood vessel growth and remodelling. Journal of cellular and molecular medicine 2014; 18:1491-1508

197. Duan J, Murohara T, Ikeda H, Sasaki K, Shintani S, Akita T, Shimada T, Imaizumi T. Hyperhomocysteinemia impairs angiogenesis in response to hindlimb ischemia. Arteriosclerosis, thrombosis, and vascular biology 2000; 20:2579-2585 
198. Jacovina AT, Deora AB, Ling $Q$, Broekman MJ, Almeida D, Greenberg CB, Marcus AJ, Smith JD, Hajjar KA. Homocysteine inhibits neoangiogenesis in mice through blockade of annexin A2-dependent fibrinolysis. The Journal of clinical investigation 2009; 119:3384-3394

199. Nagai Y, Tasaki H, Takatsu H, Nihei S, Yamashita K, Toyokawa T, Nakashima Y. Homocysteine inhibits angiogenesis in vitro and in vivo. Biochemical and biophysical research communications 2001; 281:726-731

200. Oosterbaan AM, Steegers EA, Ursem NT. The effects of homocysteine and folic acid on angiogenesis and VEGF expression during chicken vascular development. Microvascular research 2012; 83:98-104

201. Zhang $Q$, Li Q, Chen $Y$, Huang X, Yang IH, Cao L, Wu WK, Tan HM. Homocysteineimpaired angiogenesis is associated with VEGF/VEGFR inhibition. Frontiers in bioscience (Elite edition) 2012; 4:2525-2535

202. Johnson KE, Wilgus TA. Vascular Endothelial Growth Factor and Angiogenesis in the Regulation of Cutaneous Wound Repair. Advances in wound care 2014; 3:647661

203. Handy DE, Zhang Y, Loscalzo J. Homocysteine down-regulates cellular glutathione peroxidase (GPX1) by decreasing translation. The Journal of biological chemistry 2005; 280:15518-15525

204. Galasso G, Schiekofer S, Sato K, Shibata R, Handy DE, Ouchi N, Leopold JA, Loscalzo $\mathrm{J}$, Walsh K. Impaired angiogenesis in glutathione peroxidase-1-deficient mice is associated with endothelial progenitor cell dysfunction. Circulation research 2006; 98:254-261

205. Upchurch GR, Jr., Welch GN, Fabian AJ, Freedman JE, Johnson JL, Keaney JF, Jr., Loscalzo J. Homocyst(e)ine decreases bioavailable nitric oxide by a mechanism involving glutathione peroxidase. The Journal of biological chemistry 1997; 272:17012-17017

206. Eberhardt RT, Forgione MA, Cap A, Leopold JA, Rudd MA, Trolliet M, Heydrick S, Stark R, Klings ES, Moldovan NI, Yaghoubi M, Goldschmidt-Clermont PJ, Farber HW, Cohen R, Loscalzo J. Endothelial dysfunction in a murine model of mild hyperhomocyst(e)inemia. The Journal of clinical investigation 2000; 106:483-491

207. Kundu S, Kumar M, Sen U, Mishra PK, Tyagi N, Metreveli N, Lominadze D, Rodriguez W, Tyagi SC. Nitrotyrosinylation, remodeling and endothelial-myocyte uncoupling in iNOS, cystathionine beta synthase (CBS) knockouts and iNOS/CBS double knockout mice. Journal of cellular biochemistry 2009; 106:119-126

208. Kundu S, Pushpakumar S, Sen U. MMP-9- and NMDA receptor-mediated mechanism of diabetic renovascular remodeling and kidney dysfunction: hydrogen sulfide is a key modulator. Nitric oxide : biology and chemistry 2015; 46:172-185

209. Wang H, Yoshizumi M, Lai K, Tsai JC, Perrella MA, Haber E, Lee ME. Inhibition of growth and p21ras methylation in vascular endothelial cells by homocysteine but not cysteine. The Journal of biological chemistry 1997; 272:25380-25385 
210. Lentz SR, Sobey CG, Piegors DJ, Bhopatkar MY, Faraci FM, Malinow MR, Heistad DD. Vascular dysfunction in monkeys with diet-induced hyperhomocyst(e)inemia. The Journal of clinical investigation 1996; 98:24-29

211. Lee $\mathrm{ME}$, Wang $\mathrm{H}$. Homocysteine and hypomethylation. A novel link to vascular disease. Trends in cardiovascular medicine 1999; 9:49-54

212. Harker LA, Harlan JM, Ross R. Effect of sulfinpyrazone on homocysteine-induced endothelial injury and arteriosclerosis in baboons. Circulation research 1983; 53:731-739

213. Jamaluddin MD, Chen I, Yang F, Jiang X, Jan M, Liu X, Schafer Al, Durante W, Yang $X$, Wang $H$. Homocysteine inhibits endothelial cell growth via DNA hypomethylation of the cyclin A gene. Blood 2007; 110:3648-3655

214. Bodine SC, Baehr LM. Skeletal muscle atrophy and the E3 ubiquitin ligases MuRF1 and MAFbx/atrogin-1. American journal of physiology Endocrinology and metabolism 2014; 307:E469-484

215. Bonaldo $P$, Sandri M. Cellular and molecular mechanisms of muscle atrophy. Disease models \& mechanisms 2013; 6:25-39

216. Carter ME, Brunet A. FOXO transcription factors. Curr Biol 2007; 17:R113-114

217. Kang $\mathrm{SH}$, Lee HA, Kim M, Lee E, Sohn UD, Kim I. Forkhead box $\mathrm{O} 3$ plays a role in skeletal muscle atrophy through expression of E3 ubiquitin ligases MuRF-1 and atrogin-1 in Cushing's syndrome. American journal of physiology Endocrinology and metabolism 2017; 312:E495-e507

218. Gross DN, van den Heuvel AP, Birnbaum MJ. The role of FoxO in the regulation of metabolism. Oncogene 2008; 27:2320-2336

219. Huang $\mathrm{H}$, Tindall DJ. Dynamic FoxO transcription factors. Journal of cell science 2007; 120:2479-2487

220. Brunet A, Bonni A, Zigmond MJ, Lin MZ, Juo P, Hu LS, Anderson MJ, Arden KC, Blenis J, Greenberg ME. Akt promotes cell survival by phosphorylating and inhibiting a Forkhead transcription factor. Cell 1999; 96:857-868

221. Essers MA, Weijzen S, de Vries-Smits AM, Saarloos I, de Ruiter ND, Bos JL, Burgering BM. FOXO transcription factor activation by oxidative stress mediated by the small GTPase Ral and JNK. The EMBO journal 2004; 23:4802-4812

222. Wang $M C$, Bohmann $D$, Jasper $H$. JNK extends life span and limits growth by antagonizing cellular and organism-wide responses to insulin signaling. Cell 2005; 121:115-125

223. Brunet A, Bonni A, Zigmond MJ, Lin MZ, Juo P, Hu LS, Anderson MJ, Arden KC, Blenis J, Greenberg ME. Akt Promotes Cell Survival by Phosphorylating and Inhibiting a Forkhead Transcription Factor. Cell 1999; 96:857-868

224. Sandri M, Sandri C, Gilbert A, Skurk C, Calabria E, Picard A, Walsh K, Schiaffino S, Lecker SH, Goldberg AL. Foxo Transcription Factors Induce the Atrophy-Related Ubiquitin Ligase Atrogin-1 and Cause Skeletal Muscle Atrophy. Cell 2004; 117:399412

225. Filler K, Lyon D, Bennett J, McCain N, Elswick R, Lukkahatai N, Saligan LN. Association of Mitochondrial Dysfunction and Fatigue: A Review of the Literature. BBA clinical 2014; 1:12-23 
226. Myhill S, Booth NE, McLaren-Howard J. Chronic fatigue syndrome and mitochondrial dysfunction. International journal of clinical and experimental medicine 2009; 2:1-16

227. Osian G, Procopciuc L, Vlad L, lancu C, Mocan T, Mocan L. C677T and A1298C mutations in the MTHFR gene and survival in colorectal cancer. Journal of gastrointestinal and liver diseases : JGLD 2009; 18:455-460

228. Givvimani S, Narayanan N, Armaghan F, Pushpakumar S, Tyagi SC. Attenuation of conducted vasodilation in skeletal muscle arterioles during hyperhomocysteinemia. Pharmacology 2013; 91:287-296

229. Polhemus DJ, Lefer DJ. Emergence of hydrogen sulfide as an endogenous gaseous signaling molecule in cardiovascular disease. Circulation research 2014; 114:730737

230. Jiang $H$, Xiao J, Kang $B$, Zhu X, Xin N, Wang Z. PI3K/SGK1/GSK3beta signaling pathway is involved in inhibition of autophagy in neonatal rat cardiomyocytes exposed to hypoxia/reoxygenation by hydrogen sulfide. Experimental cell research 2016; 345:134-140

231. Sun X, Wang W, Dai J, Jin S, Huang J, Guo C, Wang C, Pang L, Wang Y. A Long-Term and Slow-Releasing Hydrogen Sulfide Donor Protects against Myocardial Ischemia/Reperfusion Injury. Scientific reports 2017; 7:3541

232. Saglam K, Alhan E, Turkyilmaz S, Vanizor BK, Ercin C. The anti-inflammatory effect of hydrogen sulphide on acute necrotizing pancreatitis in rats. Turkish journal of surgery 2017; 33:158-163

233. Gemici B, Elsheikh W, Feitosa KB, Costa SK, Muscara MN, Wallace JL. H2S-releasing drugs: anti-inflammatory, cytoprotective and chemopreventative potential. Nitric oxide : biology and chemistry 2015; 46:25-31

234. Xie ZZ, Liu Y, Bian JS. Hydrogen Sulfide and Cellular Redox Homeostasis. Oxidative medicine and cellular longevity 2016; 2016:6043038

235. Doeller JE, Isbell TS, Benavides G, Koenitzer J, Patel H, Patel RP, Lancaster JR, Jr., Darley-Usmar VM, Kraus DW. Polarographic measurement of hydrogen sulfide production and consumption by mammalian tissues. Analytical biochemistry 2005; 341:40-51

236. Kozich V, Krijt J, Sokolova J, Melenovska P, Jesina P, Vozdek R, Majtan T, Kraus JP. Thioethers as markers of hydrogen sulfide production in homocystinurias. Biochimie 2016; 126:14-20

237. Whiteman M, Armstrong JS, Chu SH, Jia-Ling S, Wong BS, Cheung NS, Halliwell B, Moore PK. The novel neuromodulator hydrogen sulfide: an endogenous peroxynitrite 'scavenger'? Journal of neurochemistry 2004; 90:765-768

238. Whiteman M, Cheung NS, Zhu YZ, Chu SH, Siau JL, Wong BS, Armstrong JS, Moore PK. Hydrogen sulphide: a novel inhibitor of hypochlorous acid-mediated oxidative damage in the brain? Biochemical and biophysical research communications 2005; 326:794-798

239. Greiner R, Palinkas Z, Basell K, Becher D, Antelmann H, Nagy P, Dick TP. Polysulfides link H2S to protein thiol oxidation. Antioxidants \& redox signaling 2013; 19:1749-1765 
240. Al-Magableh MR, Kemp-Harper BK, Ng HH, Miller AA, Hart JL. Hydrogen sulfide protects endothelial nitric oxide function under conditions of acute oxidative stress in vitro. Naunyn-Schmiedeberg's archives of pharmacology 2014; 387:67-74

241. Predmore BL, Lefer DJ, Gojon G. Hydrogen sulfide in biochemistry and medicine. Antioxidants \& redox signaling 2012; 17:119-140

242. Whiteman M, Li L, Kostetski I, Chu SH, Siau JL, Bhatia M, Moore PK. Evidence for the formation of a novel nitrosothiol from the gaseous mediators nitric oxide and hydrogen sulphide. Biochemical and biophysical research communications 2006; 343:303-310

243. Geng B, Chang L, Pan C, Qi Y, Zhao J, Pang Y, Du J, Tang C. Endogenous hydrogen sulfide regulation of myocardial injury induced by isoproterenol. Biochemical and biophysical research communications 2004; 318:756-763

244. Kimura $\mathrm{Y}$, Kimura $\mathrm{H}$. Hydrogen sulfide protects neurons from oxidative stress. FASEB journal : official publication of the Federation of American Societies for Experimental Biology 2004; 18:1165-1167

245. Lu M, Hu LF, Hu G, Bian JS. Hydrogen sulfide protects astrocytes against $\mathrm{H}(2) \mathrm{O}(2)$ induced neural injury via enhancing glutamate uptake. Free radical biology \& medicine 2008; 45:1705-1713

246. Tyagi N, Moshal KS, Sen U, Vacek TP, Kumar M, Hughes WM, Jr., Kundu S, Tyagi SC. $\mathrm{H} 2 \mathrm{~S}$ protects against methionine-induced oxidative stress in brain endothelial cells. Antioxidants \& redox signaling 2009; 11:25-33

247. Kesherwani V, Nelson KS, Agrawal SK. Effect of sodium hydrosulphide after acute compression injury of spinal cord. Brain research 2013; 1527:222-229

248. Huang C, Kan J, Liu X, Ma F, Tran BH, Zou Y, Wang S, Zhu YZ. Cardioprotective effects of a novel hydrogen sulfide agent-controlled release formulation of Spropargyl-cysteine on heart failure rats and molecular mechanisms. PloS one 2013; 8:e69205

249. Wang C, Wang HY, Liu ZW, Fu Y, Zhao B. Effect of endogenous hydrogen sulfide on oxidative stress in oleic acid-induced acute lung injury in rats. Chinese medical journal 2011; 124:3476-3480

250. Sen U, Basu P, Abe OA, Givvimani S, Tyagi N, Metreveli N, Shah KS, Passmore JC, Tyagi SC. Hydrogen sulfide ameliorates hyperhomocysteinemia-associated chronic renal failure. American journal of physiology Renal physiology 2009; 297:F410-419

251. Guo C, Liang F, Shah Masood W, Yan X. Hydrogen sulfide protected gastric epithelial cell from ischemia/reperfusion injury by Keap1 s-sulfhydration, MAPK dependent anti-apoptosis and NF-kappaB dependent anti-inflammation pathway. European journal of pharmacology 2014; 725:70-78

252. Cui J, Liu L, Zou J, Qiao W, Liu H, Qi Y, Yan C. Protective effect of endogenous hydrogen sulfide against oxidative stress in gastric ischemia-reperfusion injury. Experimental and therapeutic medicine 2013; 5:689-694

253. Zhu X, Tang Z, Cong B, Du J, Wang C, Wang L, Ni X, Lu J. Estrogens increase cystathionine-gamma-lyase expression and decrease inflammation and oxidative 
stress in the myocardium of ovariectomized rats. Menopause (New York, NY) 2013; 20:1084-1091

254. Fu Z, Liu X, Geng B, Fang L, Tang C. Hydrogen sulfide protects rat lung from ischemia-reperfusion injury. Life sciences 2008; 82:1196-1202

255. Wen YD, Wang H, Kho SH, Rinkiko S, Sheng X, Shen HM, Zhu YZ. Hydrogen sulfide protects HUVECs against hydrogen peroxide induced mitochondrial dysfunction and oxidative stress. PloS one 2013; 8:e53147

256. Liu YY, Nagpure BV, Wong PT, Bian JS. Hydrogen sulfide protects SH-SY5Y neuronal cells against $d$-galactose induced cell injury by suppression of advanced glycation end products formation and oxidative stress. Neurochemistry international 2013; 62:603-609

257. Benetti LR, Campos D, Gurgueira SA, Vercesi AE, Guedes CE, Santos KL, Wallace JL, Teixeira SA, Florenzano J, Costa SK, Muscara MN, Ferreira HH. Hydrogen sulfide inhibits oxidative stress in lungs from allergic mice in vivo. European journal of pharmacology 2013; 698:463-469

258. Su YW, Liang C, Jin HF, Tang XY, Han W, Chai LJ, Zhang CY, Geng B, Tang CS, Du JB. Hydrogen sulfide regulates cardiac function and structure in adriamycin-induced cardiomyopathy. Circulation journal : official journal of the Japanese Circulation Society 2009; 73:741-749

259. Wei $H$, Zhang $R$, Jin $H$, Liu D, Tang $X$, Tang C, Du J. Hydrogen sulfide attenuates hyperhomocysteinemia-induced cardiomyocytic endoplasmic reticulum stress in rats. Antioxidants \& redox signaling 2010; 12:1079-1091

260. Li C, Hu M, Wang Y, Lu H, Deng J, Yan X. Hydrogen sulfide preconditioning protects against myocardial ischemia/reperfusion injury in rats through inhibition of endo/sarcoplasmic reticulum stress. International journal of clinical and experimental pathology 2015; 8:7740-7751

261. Li X, Zhang KY, Zhang P, Chen LX, Wang L, Xie M, Wang CY, Tang XQ. Hydrogen sulfide inhibits formaldehyde-induced endoplasmic reticulum stress in PC12 cells by upregulation of SIRT-1. PloS one 2014; 9:e89856

262. Sun H, Qi L, Wang S, Li X, Li C. Hydrogen sulfide is expressed in the human and the rat cultured nucleus pulposus cells and suppresses apoptosis induced by hypoxia. PloS one 2018; 13:e0192556

263. Lin F, Liao C, Sun Y, Zhang J, Lu W, Bai Y, Liao Y, Li M, Ni X, Hou Y, Qi Y, Chen Y. Hydrogen Sulfide Inhibits Cigarette Smoke-Induced Endoplasmic Reticulum Stress and Apoptosis in Bronchial Epithelial Cells. Frontiers in pharmacology 2017; 8:675

264. Wei HJ, Xu JH, Li MH, Tang JP, Zou W, Zhang P, Wang L, Wang CY, Tang XQ. Hydrogen sulfide inhibits homocysteine-induced endoplasmic reticulum stress and neuronal apoptosis in rat hippocampus via upregulation of the BDNF-TrkB pathway. Acta pharmacologica Sinica 2014; 35:707-715

265. Luo Y, Liu X, Zheng Q, Wan X, Ouyang S, Yin Y, Sui X, Liu J, Yang X. Hydrogen sulfide prevents hypoxia-induced apoptosis via inhibition of an $\mathrm{H} 2 \mathrm{O} 2$-activated calcium signaling pathway in mouse hippocampal neurons. Biochemical and biophysical research communications 2012; 425:473-477 
266. Wu D, Luo N, Wang L, Zhao Z, Bu H, Xu G, Yan Y, Che X, Jiao Z, Zhao T, Chen J, Ji A, Li Y, Lee GD. Hydrogen sulfide ameliorates chronic renal failure in rats by inhibiting apoptosis and inflammation through ROS/MAPK and NF-kappaB signaling pathways. Scientific reports $2017 ; 7: 455$

267. Shi S, Li QS, Li H, Zhang L, Xu M, Cheng JL, Peng CH, Xu CQ, Tian Y. Anti-apoptotic action of hydrogen sulfide is associated with early JNK inhibition. Cell biology international 2009; 33:1095-1101

268. Yin $W L, H$ e JQ, Hu B, Jiang ZS, Tang XQ. Hydrogen sulfide inhibits MPP(+)-induced apoptosis in PC12 cells. Life sciences 2009; 85:269-275

269. Cai WJ, Wang MJ, Moore PK, Jin HM, Yao T, Zhu YC. The novel proangiogenic effect of hydrogen sulfide is dependent on Akt phosphorylation. Cardiovascular research 2007; 76:29-40

270. Papapetropoulos A, Pyriochou A, Altaany Z, Yang G, Marazioti A, Zhou Z, Jeschke MG, Branski LK, Herndon DN, Wang R, Szabo C. Hydrogen sulfide is an endogenous stimulator of angiogenesis. Proceedings of the National Academy of Sciences of the United States of America 2009; 106:21972-21977

271. Szabo C, Papapetropoulos A. Hydrogen sulphide and angiogenesis: mechanisms and applications. British journal of pharmacology 2011; 164:853-865

272. Wang R, Szabo C, Ichinose F, Ahmed A, Whiteman M, Papapetropoulos A. The role of $\mathrm{H} 2 \mathrm{~S}$ bioavailability in endothelial dysfunction. Trends in pharmacological sciences 2015; 36:568-578

273. Minamishima S, Bougaki M, Sips PY, Yu JD, Minamishima YA, Elrod JW, Lefer DJ, Bloch KD, Ichinose F. Hydrogen sulfide improves survival after cardiac arrest and cardiopulmonary resuscitation via a nitric oxide synthase 3-dependent mechanism in mice. Circulation 2009; 120:888-896

274. Yusof M, Kamada K, Kalogeris T, Gaskin FS, Korthuis RJ. Hydrogen sulfide triggers late-phase preconditioning in postischemic small intestine by an NO- and p38 MAPK-dependent mechanism. American journal of physiology Heart and circulatory physiology 2009; 296:H868-876

275. Bauer CC, Boyle JP, Porter KE, Peers C. Modulation of $\mathrm{Ca}(2+)$ signalling in human vascular endothelial cells by hydrogen sulfide. Atherosclerosis 2010; 209:374-380

276. Du JT, Li W, Yang JY, Tang CS, Li Q, Jin HF. Hydrogen sulfide is endogenously generated in rat skeletal muscle and exerts a protective effect against oxidative stress. Chinese medical journal 2013; 126:930-936

277. Suzuki K, Olah G, Modis K, Coletta C, Kulp G, Gero D, Szoleczky P, Chang T, Zhou Z, Wu L, Wang R, Papapetropoulos A, Szabo C. Hydrogen sulfide replacement therapy protects the vascular endothelium in hyperglycemia by preserving mitochondrial function. Proceedings of the National Academy of Sciences of the United States of America 2011; 108:13829-13834

278. Veeranki S, Tyagi SC. Role of hydrogen sulfide in skeletal muscle biology and metabolism. Nitric oxide : biology and chemistry 2015; 46:66-71

279. Lemle MD. Hypothesis: chronic fatigue syndrome is caused by dysregulation of hydrogen sulfide metabolism. Medical hypotheses 2009; 72:108-109 
280. Zhao W, Zhang J, Lu Y, Wang R. The vasorelaxant effect of $H(2) S$ as a novel endogenous gaseous K(ATP) channel opener. The EMBO journal 2001; 20:60086016

281. Tang $G$, Wu L, Liang $W$, Wang R. Direct stimulation of K(ATP) channels by exogenous and endogenous hydrogen sulfide in vascular smooth muscle cells. Molecular pharmacology 2005; 68:1757-1764

282. Matar W, Nosek TM, Wong D, Renaud J. Pinacidil suppresses contractility and preserves energy but glibenclamide has no effect during muscle fatigue. American journal of physiology Cell physiology 2000; 278:C404-416

283. Maclntosh BR, Holash RJ, Renaud JM. Skeletal muscle fatigue--regulation of excitation-contraction coupling to avoid metabolic catastrophe. Journal of cell science 2012; 125:2105-2114

284. Majumder A, Singh M, Behera J, Theilen NT, George AK, Tyagi N, Metreveli N, Tyagi SC. Hydrogen sulfide alleviates hyperhomocysteinemia-mediated skeletal muscle atrophy via mitigation of oxidative and endoplasmic reticulum stress injury. American journal of physiology Cell physiology 2018;

285. Sente $T$, Van Berendoncks AM, Fransen E, Vrints CJ, Hoymans VY. Tumor necrosis factor-alpha impairs adiponectin signalling, mitochondrial biogenesis, and myogenesis in primary human myotubes cultures. American journal of physiology Heart and circulatory physiology 2016; 310:H1164-1175

286. Schalinske KL, Smazal AL. Homocysteine imbalance: a pathological metabolic marker. Advances in nutrition (Bethesda, Md) 2012; 3:755-762

287. Derijard B, Hibi M, Wu IH, Barrett T, Su B, Deng T, Karin M, Davis RJ. JNK1: a protein kinase stimulated by UV light and Ha-Ras that binds and phosphorylates the c-Jun activation domain. Cell 1994; 76:1025-1037

288. Hibi M, Lin A, Smeal T, Minden A, Karin M. Identification of an oncoprotein- and UV-responsive protein kinase that binds and potentiates the c-Jun activation domain. Genes \& development 1993; 7:2135-2148

289. Davis RJ. Signal transduction by the JNK group of MAP kinases. Cell 2000; 103:239252

290. Chang L, Karin M. Mammalian MAP kinase signalling cascades. Nature 2001; 410:37-40

291. Dhanasekaran DN, Johnson GL. MAPKs: function, regulation, role in cancer and therapeutic targeting. Oncogene 2007; 26:3097-3099

292. Raman $M$, Chen $W$, Cobb MH. Differential regulation and properties of MAPKs. Oncogene 2007; 26:3100-3112

293. Turjanski AG, Vaque JP, Gutkind JS. MAP kinases and the control of nuclear events. Oncogene 2007; 26:3240-3253

294. Johnson GL, Nakamura K. The c-jun kinase/stress-activated pathway: regulation, function and role in human disease. Biochimica et biophysica acta 2007; 1773:1341-1348

295. Kaminska B. Molecular characterization of inflammation-induced JNK/c-Jun signaling pathway in connection with tumorigenesis. Methods in molecular biology (Clifton, NJ) 2009; 512:249-264 
296. Fan M, Chambers TC. Role of mitogen-activated protein kinases in the response of tumor cells to chemotherapy. Drug resistance updates : reviews and commentaries in antimicrobial and anticancer chemotherapy 2001; 4:253-267

297. Ishizuka T, Terada N, Gerwins P, Hamelmann E, Oshiba A, Fanger GR, Johnson GL, Gelfand EW. Mast cell tumor necrosis factor alpha production is regulated by MEK kinases. Proceedings of the National Academy of Sciences of the United States of America 1997; 94:6358-6363

298. Nishina $H$, Bachmann $M$, Oliveira-dos-Santos AJ, Kozieradzki I, Fischer $K D$, Odermatt B, Wakeham A, Shahinian A, Takimoto H, Bernstein A, Mak TW, Woodgett JR, Ohashi PS, Penninger JM. Impaired CD28-mediated interleukin 2 production and proliferation in stress kinase SAPK/ERK1 kinase (SEK1)/mitogenactivated protein kinase kinase 4 (MKK4)-deficient T lymphocytes. The Journal of experimental medicine 1997; 186:941-953

299. Whitmarsh AJ, Davis RJ. Transcription factor AP-1 regulation by mitogen-activated protein kinase signal transduction pathways. Journal of molecular medicine (Berlin, Germany) 1996; 74:589-607

300. Londhe P, Guttridge DC. Inflammation induced loss of skeletal muscle. Bone 2015; 80:131-142

301. Kalani A, Kamat PK, Givvimani S, Brown K, Metreveli N, Tyagi SC, Tyagi N. Nutriepigenetics ameliorates blood-brain barrier damage and neurodegeneration in hyperhomocysteinemia: role of folic acid. Journal of molecular neuroscience : $\mathrm{MN}$ 2014; 52:202-215

302. Kamat PK, Kalani A, Givvimani S, Sathnur PB, Tyagi SC, Tyagi N. Hydrogen sulfide attenuates neurodegeneration and neurovascular dysfunction induced by intracerebral-administered homocysteine in mice. Neuroscience 2013; 252:302319

303. Kamath AF, Chauhan AK, Kisucka J, Dole VS, Loscalzo J, Handy DE, Wagner DD. Elevated levels of homocysteine compromise blood-brain barrier integrity in mice. Blood 2006; 107:591-593

304. Sudduth TL, Powell DK, Smith CD, Greenstein A, Wilcock DM. Induction of hyperhomocysteinemia models vascular dementia by induction of cerebral microhemorrhages and neuroinflammation. Journal of cerebral blood flow and metabolism : official journal of the International Society of Cerebral Blood Flow and Metabolism 2013; 33:708-715

305. Kumar M, Tyagi N, Moshal KS, Sen U, Kundu S, Mishra PK, Givvimani S, Tyagi SC. Homocysteine decreases blood flow to the brain due to vascular resistance in carotid artery. Neurochemistry international 2008; 53:214-219

306. Rio DC, Ares M, Jr., Hannon GJ, Nilsen TW. Purification of RNA using TRIzol (TRI reagent). Cold Spring Harbor protocols 2010; 2010:pdb.prot5439

307. Chaturvedi P, Kalani A, Givvimani S, Kamat PK, Familtseva A, Tyagi SC. Differential regulation of DNA methylation versus histone acetylation in cardiomyocytes during $\mathrm{HHcy}$ in vitro and in vivo: an epigenetic mechanism. Physiological genomics $2014 ; 46: 245-255$ 
308. Puntel RL, Roos DH, Grotto D, Garcia SC, Nogueira CW, Rocha JB. Antioxidant properties of Krebs cycle intermediates against malonate pro-oxidant activity in vitro: a comparative study using the colorimetric method and HPLC analysis to determine malondialdehyde in rat brain homogenates. Life sciences 2007 ; 81:5162

309. Mosmann T. Rapid colorimetric assay for cellular growth and survival: application to proliferation and cytotoxicity assays. Journal of immunological methods 1983; 65:55-63

310. Lentz SR, Sadler JE. Homocysteine inhibits von Willebrand factor processing and secretion by preventing transport from the endoplasmic reticulum. Blood 1993; 81:683-689

311. Outinen PA, Sood SK, Liaw PC, Sarge KD, Maeda N, Hirsh J, Ribau J, Podor TJ, Weitz $\mathrm{JI}$, Austin RC. Characterization of the stress-inducing effects of homocysteine. The Biochemical journal 1998; 332 ( Pt 1):213-221

312. Roy PK, Rashid F, Bragg J, Ibdah JA. Role of the JNK signal transduction pathway in inflammatory bowel disease. World journal of gastroenterology 2008; 14:200-202

313. Oudi ME, Aouni Z, Mazigh C, Khochkar R, Gazoueni E, Haouela H, Machghoul S. Homocysteine and markers of inflammation in acute coronary syndrome. Experimental and clinical cardiology 2010; 15:e25-28

314. Gori AM, Corsi AM, Fedi S, Gazzini A, Sofi F, Bartali B, Bandinelli S, Gensini GF, Abbate $R$, Ferrucci L. A proinflammatory state is associated with hyperhomocysteinemia in the elderly. The American journal of clinical nutrition 2005; 82:335-341

315. Holven KB, Aukrust P, Retterstol K, Hagve TA, Morkrid L, Ose L, Nenseter MS. Increased levels of C-reactive protein and interleukin-6 in hyperhomocysteinemic subjects. Scandinavian journal of clinical and laboratory investigation 2006; 66:4554

316. Ponugoti B, Dong G, Graves DT. Role of forkhead transcription factors in diabetesinduced oxidative stress. Experimental diabetes research 2012; 2012:939751

317. Majumder A, Singh M, George AK, Behera J, Tyagi N, Tyagi SC. Hydrogen sulfide improves postischemic neoangiogenesis in the hind limb of cystathionine-betasynthase mutant mice via PPAR-gamma/VEGF axis. Physiological reports 2018; 6:e13858

318. Zou T, Liu WJ, Li SD, Zhou W, Yang JF, Zou CG. TRB3 mediates homocysteineinduced inhibition of endothelial cell proliferation. Journal of cellular physiology 2011; 226:2782-2789

319. Upchurch GR, Jr., Welch GN, Fabian AJ, Pigazzi A, Keaney JF, Jr., Loscalzo J. Stimulation of endothelial nitric oxide production by homocyst(e)ine. Atherosclerosis 1997; 132:177-185

320. Singh M, George AK, Homme RP, Majumder A, Laha A, Sandhu HS, Tyagi SC. Circular RNAs profiling in the cystathionine-beta-synthase mutant mouse reveals novel gene targets for hyperhomocysteinemia induced ocular disorders. Experimental eye research 2018; 174:80-92 
321. Carmeliet $P$, Jain RK. Molecular mechanisms and clinical applications of angiogenesis. Nature 2011; 473:298-307

322. Duran CL, Howell DW, Dave JM, Smith RL, Torrie ME, Essner JJ, Bayless KJ. Molecular Regulation of Sprouting Angiogenesis. Comprehensive Physiology 2017; 8:153-235

323. Biscetti F, Gaetani E, Flex A, Aprahamian T, Hopkins T, Straface G, Pecorini G, Stigliano E, Smith RC, Angelini F, Castellot JJ, Jr., Pola R. Selective activation of peroxisome proliferator-activated receptor (PPAR)alpha and PPAR gamma induces neoangiogenesis through a vascular endothelial growth factor-dependent mechanism. Diabetes 2008; 57:1394-1404

324. Chawla A, Schwarz EJ, Dimaculangan DD, Lazar MA. Peroxisome proliferatoractivated receptor (PPAR) gamma: adipose-predominant expression and induction early in adipocyte differentiation. Endocrinology 1994; 135:798-800

325. Tontonoz P, Hu E, Spiegelman BM. Regulation of adipocyte gene expression and differentiation by peroxisome proliferator activated receptor gamma. Curr Opin Genet Dev 1995; 5:571-576

326. Murphy GJ, Holder JC. PPAR-gamma agonists: therapeutic role in diabetes, inflammation and cancer. Trends Pharmacol Sci 2000; 21:469-474

327. Costa V, Gallo MA, Letizia F, Aprile M, Casamassimi A, Ciccodicola A. PPARG: Gene Expression Regulation and Next-Generation Sequencing for Unsolved Issues. PPAR Res 2010; 2010

328. Kroll J, Waltenberger J. VEGF-A induces expression of eNOS and iNOS in endothelial cells via VEGF receptor-2 (KDR). Biochemical and biophysical research communications 1998; 252:743-746

329. George AK, Singh M, Homme RP, Majumder A, Sandhu HS, Tyagi SC. A hypothesis for treating inflammation and oxidative stress with hydrogen sulfide during agerelated macular degeneration. International journal of ophthalmology 2018; 11:881-887

330. Shefa U, Kim MS, Jeong NY, Jung J. Antioxidant and Cell-Signaling Functions of Hydrogen Sulfide in the Central Nervous System. Oxidative medicine and cellular longevity 2018; 2018:1873962

331. Cai J, Shi X, Wang H, Fan J, Feng Y, Lin X, Yang J, Cui Q, Tang C, Xu G, Geng B. Cystathionine gamma lyase-hydrogen sulfide increases peroxisome proliferatoractivated receptor gamma activity by sulfhydration at C139 site thereby promoting glucose uptake and lipid storage in adipocytes. Biochimica et biophysica acta 2016; 1861:419-429

332. Bhargava S, Pushpakumar S, Metreveli N, Givvimani S, Tyagi SC. MMP-9 gene ablation mitigates hyperhomocystenemia-induced cognition and hearing dysfunction. Molecular biology reports 2014; 41:4889-4898

333. Givvimani S, Sen U, Tyagi N, Munjal C, Tyagi SC. X-ray imaging of differential vascular density in MMP-9-/-, PAR-1-/+, hyperhomocysteinemic (CBS-/+) and diabetic (Ins2-/+) mice. Archives of physiology and biochemistry 2011; 117:1-7

334. Machens HG, Grzybowski S, Bucsky B, Spanholtz T, Niedworok C, Maichle A, Stockelhuber B, Condurache A, Liu F, Egana JT, Kaun M, Mailander P, Aach T. A 
technique to detect and to quantify fasciocutaneous blood vessels in small laboratory animals ex vivo. The Journal of surgical research 2006; 131:91-96

335. Tan H, Jiang X, Yang F, Li Z, Liao D, Trial J, Magera MJ, Durante W, Yang X, Wang H. Hyperhomocysteinemia inhibits post-injury reendothelialization in mice. Cardiovascular research 2006; 69:253-262

336. Li HB, Ge YK, Zhang L, Zheng XX. Astragaloside IV improved barrier dysfunction induced by acute high glucose in human umbilical vein endothelial cells. Life sciences 2006; 79:1186-1193

337. Ozsvari B, Puskas LG, Nagy LI, Kanizsai I, Gyuris M, Madacsi R, Feher LZ, Gero D, Szabo C. A cell-microelectronic sensing technique for the screening of cytoprotective compounds. International journal of molecular medicine 2010; 25:525-530

338. Lindner V, Fingerle J, Reidy MA. Mouse model of arterial injury. Circulation research 1993; 73:792-796

339. Rosen ED, Raymond S, Zollman A, Noria F, Sandoval-Cooper M, Shulman A, Merz $\mathrm{JL}$, Castellino FJ. Laser-induced noninvasive vascular injury models in mice generate platelet- and coagulation-dependent thrombi. The American journal of pathology 2001; 158:1613-1622

340. Carmeliet $P$, Moons $L$, Collen D. Mouse models of angiogenesis, arterial stenosis, atherosclerosis and hemostasis. Cardiovascular research 1998; 39:8-33

341. Norton KA, Popel AS. Effects of endothelial cell proliferation and migration rates in a computational model of sprouting angiogenesis. Scientific reports 2016; 6:36992

342. Carmeliet P. Mechanisms of angiogenesis and arteriogenesis. Nature medicine 2000; 6:389-395

343. Liu W, Ahmad SA, Reinmuth N, Shaheen RM, Jung YD, Fan F, Ellis LM. Endothelial cell survival and apoptosis in the tumor vasculature. Apoptosis : an international journal on programmed cell death 2000; 5:323-328

344. Isner JM, Asahara T. Angiogenesis and vasculogenesis as therapeutic strategies for postnatal neovascularization. The Journal of clinical investigation 1999; 103:12311236

345. Bosch-Marce M, Pola R, Wecker AB, Silver M, Weber A, Luedemann C, Curry C, Murayama T, Kearney M, Yoon YS, Malinow MR, Asahara T, Isner JM, Losordo DW. Hyperhomocyst(e)inemia impairs angiogenesis in a murine model of limb ischemia. Vascular medicine (London, England) 2005; 10:15-22

346. Mustafa AK, Gadalla MM, Snyder SH. Signaling by gasotransmitters. Science signaling 2009; 2:re2

347. Szabo C. Gaseotransmitters: new frontiers for translational science. Science translational medicine 2010; 2:59ps54

348. Morbidelli L, Donnini S, Ziche M. Role of nitric oxide in the modulation of angiogenesis. Current pharmaceutical design 2003; 9:521-530

349. Dimmeler S, Fleming I, Fisslthaler B, Hermann C, Busse R, Zeiher AM. Activation of nitric oxide synthase in endothelial cells by Akt-dependent phosphorylation. Nature 1999; 399:601-605 
350. Topal G, Brunet A, Millanvoye E, Boucher JL, Rendu F, Devynck MA, David-Dufilho $M$. Homocysteine induces oxidative stress by uncoupling of NO synthase activity through reduction of tetrahydrobiopterin. Free radical biology \& medicine 2004; 36:1532-1541

351. Osipov RM, Robich MP, Feng J, Liu Y, Clements RT, Glazer HP, Sodha NR, Szabo C, Bianchi C, Sellke FW. Effect of hydrogen sulfide in a porcine model of myocardial ischemia-reperfusion: comparison of different administration regimens and characterization of the cellular mechanisms of protection. Journal of cardiovascular pharmacology 2009; 54:287-297

352. Coletta C, Papapetropoulos A, Erdelyi K, Olah G, Modis K, Panopoulos P, Asimakopoulou A, Gero D, Sharina I, Martin E, Szabo C. Hydrogen sulfide and nitric oxide are mutually dependent in the regulation of angiogenesis and endotheliumdependent vasorelaxation. Proceedings of the National Academy of Sciences of the United States of America 2012; 109:9161-9166

353. Laha A, Majumder A, Singh M, Tyagi SC. Connecting homocysteine and obesity through pyroptosis, gut microbiome, epigenetics, peroxisome proliferatoractivated receptor gamma, and zinc finger protein 407. Can J Physiol Pharmacol 2018:1-6

354. Elhomsy

G. Homocysteine. 2014; https://emedicine.medscape.com/article/2085682overview?pa=vl23pqAfDmEG7n\%2BdAxiOPJ3jrJV0smIW60Pa51FEXjPCmCecxkcQ Stf4KAEFW\%2FEgLY6wvQDulPoJO6m19fR0Z\%2BejCO3Rk4DWsD37DrSZWvU\%3D $\#$ \#1.

355. Gonin JM, Wilcox CS. Chapter 64 - Hyperhomocysteinemia. In: Wilcox CS, ed. Therapy in Nephrology \& Hypertension (Third Edition). Philadelphia: W.B. Saunders; 2008:725-730.

356. Yudkoff M. Chapter 42 - Disorders of Amino Acid Metabolism. In: Brady ST, Siegel GJ, Albers RW, Price DL, eds. Basic Neurochemistry (Eighth Edition). New York: Academic Press; 2012:737-754.

357. Kalra B, Ghose S, Sood N. Homocystinuria with bilateral absolute glaucoma. Indian Journal of Ophthalmology 1985; 33:195-197

358. Bodine SC, Latres E, Baumhueter S, Lai VK-M, Nunez L, Clarke BA, Poueymirou WT, Panaro FJ, Na E, Dharmarajan K, Pan Z-Q, Valenzuela DM, DeChiara TM, Stitt TN, Yancopoulos GD, Glass DJ. Identification of Ubiquitin Ligases Required for Skeletal Muscle Atrophy. Science 2001; 294:1704-1708

359. Brooks N, Myburgh K. Skeletal muscle wasting with disuse atrophy is multidimensional: the response and interaction of myonuclei, satellite cells and signaling pathways. Frontiers in Physiology 2014; 5

360. Wang Y, Zhou Y, Graves DT. FOXO Transcription Factors: Their Clinical Significance and Regulation. BioMed Research International 2014; 2014:13

361. Lagoutte E, Mimoun S, Andriamihaja M, Chaumontet C, Blachier F, Bouillaud F. Oxidation of hydrogen sulfide remains a priority in mammalian cells and causes reverse electron transfer in colonocytes. Biochim Biophys Acta 2010; 1797:15001511 
362. Morris AA, Kozich V, Santra S, Andria G, Ben-Omran TI, Chakrapani AB, Crushell E, Henderson MJ, Hochuli M, Huemer M, Janssen MC, Maillot F, Mayne PD, McNulty J, Morrison TM, Ogier H, O'Sullivan S, Pavlikova M, de Almeida IT, Terry A, Yap S, Blom HJ, Chapman KA. Guidelines for the diagnosis and management of cystathionine beta-synthase deficiency. J Inherit Metab Dis 2017; 40:49-74

363. Hwee DT, Kennedy A, Ryans J, Russell AJ, Jia Z, Hinken AC, Morgans DJ, Malik FI, Jasper JR. Fast skeletal muscle troponin activator tirasemtiv increases muscle function and performance in the B6SJL-SOD1G93A ALS mouse model. PloS one 2014; 9:e96921

364. Matsuo N, Takao K, Nakanishi K, Yamasaki N, Tanda K, Miyakawa T. Behavioral profiles of three C57BL/6 substrains. Frontiers in behavioral neuroscience 2010; 4:29

365. Damjanov I. Chapter 21 - Skeletal Muscles. Pathology Secrets (Third Edition). Philadelphia: Mosby; 2009:434-447.

366. Health P. Skeletal Muscle.

367. Britannica TEoE. Skeletal muscle. [Website]. 2018; https://www.britannica.com/science/skeletal-muscle. Accessed March 07, 2018, 2018.

368. Mann CJ, Perdiguero E, Kharraz Y, Aguilar S, Pessina P, Serrano AL, Munoz-Canoves $P$. Aberrant repair and fibrosis development in skeletal muscle. Skeletal muscle 2011; $1: 21$

369. Hay N. Interplay between FOXO, TOR, and Akt. Biochimica et biophysica acta 2011; 1813:1965-1970

370. Matsuzaki H, Daitoku H, Hatta M, Tanaka K, Fukamizu A. Insulin-induced phosphorylation of FKHR (Foxo1) targets to proteasomal degradation. Proceedings of the National Academy of Sciences 2003; 100:11285-11290

371. Aoki $\mathrm{M}$, Jiang $\mathrm{H}$, Vogt PK. Proteasomal degradation of the FoxO1 transcriptional regulator in cells transformed by the P3k and Akt oncoproteins. Proceedings of the National Academy of Sciences of the United States of America 2004; 101:13613-13617

372. Greer EL, Brunet A. FOXO transcription factors in ageing and cancer. Acta physiologica (Oxford, England) 2008; 192:19-28

373. van der Horst $A$, de Vries-Smits $A M M$, Brenkman $A B$, van Triest $M H$, van den Broek $\mathrm{N}$, Colland F, Maurice MM, Burgering BMT. FOXO4 transcriptional activity is regulated by monoubiquitination and USP7/HAUSP. Nature Cell Biology 2006; 8:1064

374. Huang $\mathrm{H}$, Tindall DJ. Regulation of FOXO protein stability via ubiquitination and proteasome degradation. Biochim Biophys Acta 2011; 1813:1961-1964

375. McLoughlin TJ, Smith SM, DeLong AD, Wang H, Unterman TG, Esser KA. FoxO1 induces apoptosis in skeletal myotubes in a DNA-binding-dependent manner. American journal of physiology Cell physiology 2009; 297:C548-555

376. Sandri M, Lin J, Handschin C, Yang W, Arany ZP, Lecker SH, Goldberg AL, Spiegelman BM. PGC-1alpha protects skeletal muscle from atrophy by suppressing 
FoxO3 action and atrophy-specific gene transcription. Proceedings of the National Academy of Sciences of the United States of America 2006; 103:16260-16265

377. Senf SM, Dodd SL, McClung JM, Judge AR. Hsp70 overexpression inhibits NFkappaB and Foxo3a transcriptional activities and prevents skeletal muscle atrophy. FASEB journal : official publication of the Federation of American Societies for Experimental Biology 2008; 22:3836-3845

378. Waddell DS, Baehr LM, van den Brandt J, Johnsen SA, Reichardt HM, Furlow JD, Bodine SC. The glucocorticoid receptor and FOXO1 synergistically activate the skeletal muscle atrophy-associated MuRF1 gene. American journal of physiology Endocrinology and metabolism 2008; 295:E785-797

379. Baehr LM, Furlow JD, Bodine SC. Muscle sparing in muscle RING finger 1 null mice: response to synthetic glucocorticoids. The Journal of physiology 2011; 589:47594776

380. Cho JE, Fournier M, Da X, Lewis MI. Time course expression of Foxo transcription factors in skeletal muscle following corticosteroid administration. Journal of applied physiology (Bethesda, Md : 1985) 2010; 108:137-145

381. Furuyama T, Kitayama K, Yamashita H, Mori N. Forkhead transcription factor FOXO1 (FKHR)-dependent induction of PDK4 gene expression in skeletal muscle during energy deprivation. The Biochemical journal 2003; 375:365-371

382. Lecker SH, Jagoe RT, Gilbert A, Gomes M, Baracos V, Bailey J, Price SR, Mitch WE, Goldberg AL. Multiple types of skeletal muscle atrophy involve a common program of changes in gene expression. FASEB journal : official publication of the Federation of American Societies for Experimental Biology 2004; 18:39-51

383. Sacheck JM, Hyatt JP, Raffaello A, Jagoe RT, Roy RR, Edgerton VR, Lecker SH, Goldberg AL. Rapid disuse and denervation atrophy involve transcriptional changes similar to those of muscle wasting during systemic diseases. FASEB journal : official publication of the Federation of American Societies for Experimental Biology 2007; 21:140-155

384. Lagirand-Cantaloube J, Cornille K, Csibi A, Batonnet-Pichon S, Leibovitch MP, Leibovitch SA. Inhibition of atrogin-1/MAFbx mediated MyoD proteolysis prevents skeletal muscle atrophy in vivo. PloS one 2009; 4:e4973

385. Clarke BA, Drujan D, Willis MS, Murphy LO, Corpina RA, Burova E, Rakhilin SV, Stitt TN, Patterson C, Latres E, Glass DJ. The E3 Ligase MuRF1 degrades myosin heavy chain protein in dexamethasone-treated skeletal muscle. Cell metabolism 2007; 6:376-385

386. Moriscot AS, Baptista IL, Bogomolovas J, Witt C, Hirner S, Granzier H, Labeit S. MuRF1 is a muscle fiber-type II associated factor and together with MuRF2 regulates type-II fiber trophicity and maintenance. Journal of structural biology 2010; 170:344-353

387. Garcia A, Zanibbi K. Homocysteine and cognitive function in elderly people. CMAJ : Canadian Medical Association journal = journal de l'Association medicale canadienne 2004; 171:897-904

388. Kuo HK, Sorond FA, Chen JH, Hashmi A, Milberg WP, Lipsitz LA. The role of homocysteine in multisystem age-related problems: a systematic review. The 
journals of gerontology Series A, Biological sciences and medical sciences 2005; 60:1190-1201

389. Kovesdy CP, Anderson JE, Kalantar-Zadeh K. Inverse association between lipid levels and mortality in men with chronic kidney disease who are not yet on dialysis: effects of case mix and the malnutrition-inflammation-cachexia syndrome. Journal of the American Society of Nephrology : JASN 2007; 18:304311

390. Kalantar-Zadeh K. Recent advances in understanding the malnutritioninflammation-cachexia syndrome in chronic kidney disease patients: What is next? Seminars in dialysis 2005; 18:365-369

391. Kumar A, Palfrey HA, Pathak R, Kadowitz PJ, Gettys TW, Murthy SN. The metabolism and significance of homocysteine in nutrition and health. Nutrition \& metabolism 2017; 14:78 


\section{LIST OF ABBREVIATIONS AND SYMBOLS}

\begin{tabular}{|c|c|}
\hline HHcy: & Hyperhomocysteinemia \\
\hline CBS: & Cystathionine- $\beta$-synthase \\
\hline CSE: & Cystathionine $\mathrm{Y}$-lyase \\
\hline WT: & Wild-type \\
\hline Met: & Methionine \\
\hline NaHS: & Sodium hydrogen sulfide \\
\hline UPR: & Unfolded protein response \\
\hline IRE1a: & Inositol-requiring enzyme-1 $\alpha$ \\
\hline XBP-1: & X-box binding protein-1 \\
\hline PERK: & PRKR-like ER kinase \\
\hline ATF6: & Activating transcription factor- 6 \\
\hline TRAF2: & TNF receptor-associated factor-2 \\
\hline JNK: & C-Jun N-terminal kinase \\
\hline ASK1: & Apoptosis signal-regulating kinase- 1 \\
\hline FOXO: & Forkhead box protein $\mathrm{O}$ \\
\hline MuRF1: & Muscle RING-finger protein-1 \\
\hline MAFBx: & Muscle atrophy F-box \\
\hline MDA: & Malondialdehyde \\
\hline ROS: & Reactive oxygen species \\
\hline ER: & Endoplasmic reticulum \\
\hline DCFDA: & 2', 7'-dichlorofluorescin diacetate \\
\hline ELISA: & Enzyme-linked immunosorbent assay \\
\hline MHC-I: & Myosin heavy chain isoform (MHC)-I \\
\hline
\end{tabular}




$\begin{array}{ll}\mathrm{H}_{2} \mathrm{~S}: & \text { Hydrogen sulfide } \\ \text { FAL: } & \text { Femoral artery ligation } \\ \text { HIF1a: } & \text { Hypoxia-Inducible Factor } 1 \alpha \\ \text { PPAR-Y: } & \text { Peroxisome proliferator-activated receptor-y } \\ \text { VEGF: } & \text { Vascular endothelial growth factor } \\ \text { eNOS: } & \text { Endothelial nitric oxide synthase } \\ \text { EC: } & \text { Endothelial cell } \\ \text { DNMT: } & \text { DNA methyltransferase } \\ \text { MS: } & \text { Methionine synthase } \\ \text { SAH: } & \text { S-adenosyl Hcy } \\ \text { THF: } & \text { Tetrahydrofolate } \\ \text { GPx: } & \text { Glutathione peroxidase } \\ \text { SOD: } & \text { Superoxide dismutase } \\ \text { CAT: } & \text { Catalase }\end{array}$




\section{Appendix I}

Table 4. Physiological parameters of experimental mice.

\begin{tabular}{|c|c|c|c|c|}
\hline $\begin{array}{c}\text { Parameters } \\
\text { (mean+/-s.e.m) }\end{array}$ & WT & CBS+Met & CBS+Met+NaHS & WT+NaHS \\
\hline BW 0 weeks $(\mathrm{gm})$ & $23.614+0.7566$ & $20.16045+0.4921$ & $20.70098+0.7799$ & $24.13964+0.5933$ \\
\hline BW 4 weeks $(\mathrm{gm})$ & $27.125+0.6500$ & $22.563+0.6325$ & $24.568+0.6852$ & $28.214+0.7481$ \\
\hline BW 8 weeks $(\mathrm{gm})$ & $29.03981+0.4557$ & $23.15864+0.7504$ & $26.45705+0.5716$ & $30.54376+0.6692$ \\
\hline Tibia length (mm) & $15.36+1.2923$ & $14.98+1.1214$ & $14.59+1.1241$ & $15.45+1.2832$ \\
\hline Gastroc (gm) & $0.14+0.0094$ & $0.1+0.0098$ & $0.121+0.0094$ & $0.142+0.0064$ \\
\hline Quad (gm) & $0.189+0.0111$ & $0.155+0.0111$ & $0.162+0.0111$ & $0.191+0.0111$ \\
\hline TA (gm) & $0.053+0.0116$ & $0.049+0.0094$ & $0.05+0.0116$ & $0.054+0.0116$ \\
\hline EDL (gm) & $0.019+0.0013$ & $0.017+0.0018$ & $0.017+0.0013$ & $0.02+0.0013$ \\
\hline Soleus (gm) & $0.013+0.0053$ & $0.009+0.0004$ & $0.01+0.0054$ & $0.006+0.0054$ \\
\hline
\end{tabular}

Note: BW: Body weight

Table 5: Results from exercise capacity test.

\begin{tabular}{|c|c|c|c|c|}
\hline \multicolumn{5}{|c|}{ Distance traveled in swimming test (m) } \\
\hline Srl\# & WT & CBS+Met & CBS+Met+NaHS & WT+NaHS \\
\hline 1 & 26.28 & 12.36 & 18.33 & 25.36 \\
\hline 2 & 20.53 & 9.63 & 23.34 & 28.45 \\
\hline 3 & 30.54 & 17.33 & 19.34 & 19.52 \\
\hline 4 & 31.33 & 13.33 & 16.33 & 31.03 \\
\hline 5 & 29.36 & 18.35 & 22.33 & 29.37 \\
\hline 6 & 20.37 & 10.35 & 15.36 & 24.32 \\
\hline 7 & 27.33 & 13.03 & 29.65 & 26.35 \\
\hline 8 & 23.21 & 15.33 & 24.33 & 24.99 \\
\hline 9 & 19.85 & 20.64 & 18.63 & 22.75 \\
\hline Mean+/-s.e.m. & $25.4216+/-1.5198$ & $14.4820+/-1.2372$ & $20.8475+/-1.5003$ & $25.7928+/-1.1734$ \\
\hline \multicolumn{5}{|c|}{ Time spent in swimming test (mins) } \\
\hline \\
\hline 1 & WT & CBS+Met & CBS+Met+NaHS & WT+NaHS \\
\hline 2 & 23.15 & 15.55 & 16.67 & 23.67 \\
\hline 3 & 17.52 & 14.10 & 21.79 & 27.17 \\
\hline 4 & 29.02 & 16.69 & 15.00 & 19.16 \\
\hline 5 & 22.53 & 13.63 & 17.65 & 28.37 \\
\hline 6 & 22.63 & 17.32 & 18.36 & 22.37 \\
\hline 7 & 25.36 & 18.36 & 22.30 & 24.30 \\
\hline
\end{tabular}




\begin{tabular}{|c|c|c|c|c|}
\hline 8 & 28.63 & 19.33 & 25.65 & 26.33 \\
\hline 9 & 22.37 & 12.75 & 27.52 & 23.20 \\
\hline Mean+/-s.e.m. & $23.90+/-1.1657$ & $15.86+/-0.74266$ & $21.03+/-1.44570$ & $24.80+/-1.03671$ \\
\hline \multicolumn{5}{|c|}{ Latency to fall in Rolla rod grip test (Secs) } \\
\hline & WT & CBS+Met & $\mathrm{CBS}+\mathrm{Met}+\mathrm{NaHS}$ & WT+NaHS \\
\hline 1 & 253 & 153 & 200 & 251 \\
\hline 2 & 263 & 163 & 210 & 249 \\
\hline 3 & 230 & 186 & 199 & 246 \\
\hline 4 & 198 & 210 & 176 & 198 \\
\hline 5 & 200 & 146 & 230 & 202 \\
\hline 6 & 263 & 163 & 156 & 193 \\
\hline 7 & 257 & 155 & 277 & 245 \\
\hline 8 & 243 & 187 & 244 & 168 \\
\hline 9 & 271 & 139 & 261 & 253 \\
\hline Mean+/-s.e.m. & $242.36+/-9.08560$ & $167.21+/-7.65148$ & $217.27+/-13.1677$ & $223.14+/-10.7941$ \\
\hline \multicolumn{5}{|c|}{ Bioseb Grip strength test-strength/BW (g-force/gm) } \\
\hline & WT & CBS+Met & $\mathrm{CBS}+\mathrm{Met}+\mathrm{NaHS}$ & $\mathrm{WT}+\mathrm{NaHS}$ \\
\hline 1 & 35.10 & 15.15 & 23.10 & 40.42 \\
\hline 2 & 30.02 & 10.15 & 35.12 & 46.15 \\
\hline 3 & 40.12 & 21.12 & 31.45 & 30.13 \\
\hline 4 & 35.36 & 13.85 & 21.14 & 36.45 \\
\hline 5 & 39.37 & 25.36 & 30.30 & 40.52 \\
\hline 6 & 44.35 & 35.63 & 40.37 & 39.33 \\
\hline 7 & 36.33 & 19.87 & 33.36 & 33.33 \\
\hline 8 & 31.79 & 24.63 & 37.32 & 28.33 \\
\hline 9 & 29.32 & 22.32 & 43.33 & 43.27 \\
\hline Mean+/-s.e.m. & $35.75+/-1.65373$ & $20.90+/-2.50639$ & $32.83+/-2.44996$ & $37.55+/-1.99293$ \\
\hline
\end{tabular}




\title{
Appendix II
}

\section{PUBLICATIONS DURING MY PHD THESIS WORK}

Am J Physiol Cell Physiol 315: C609-C622, 2018. First published August 15, 2018; doi:10.1152/ajpcell.00147.2018.

\section{RESEARCH ARTICLE $\mid$ Endoplasmic Reticulum Functions in Cell Physiology and}

\section{Disease}

\section{Hydrogen sulfide alleviates hyperhomocysteinemia-mediated skeletal muscle atrophy via mitigation of oxidative and endoplasmic reticulum stress injury}

\author{
Avisek Majumder, ${ }^{1,2}$ Mahavir Singh, ${ }^{1}$ Jyotirmaya Behera, ${ }^{1}$ Nicholas T. Theilen, ${ }^{1}$ Akash K. George, \\ Neetu Tyagi, ${ }^{1}$ Naira Metreveli, ${ }^{1}$ and Suresh C Tyagi ${ }^{1}$ \\ ${ }^{1}$ Department of Physiology, University of Louisville School of Medicine, Louisville, Kentucky; and ${ }^{2}$ Department of \\ Biochemistry and Molecular Genetics, University of Louisville School of Medicine, Louisville, Kentucky \\ Submitted 17 April 2018; accepted in final form 1 August 2018
}

Majumder A, Singh M, Behera J, Theilen NT, George AK, Tyagi N, Metreveli N, Tyagi SC. Hydrogen sulfide alleviates hyperhomocysteinemia-mediated skeletal muscle atrophy via mitigation of oxidative and endoplasmic reticulum stress injury. Am J Physiol Cell Physiol 315: C609-C622, 2018. First published August 15, 2018; doi:10.1152/ajpcell.00147.2018. - Although hyperhomocysteinemia (HHcy) occurs because of the deficiency in cystathionine- $\beta$-synthase (CBS) causing skeletal muscle dysfunction, it is still unclear whether (ER) stress, or both. Nevertheless, there is no treatment option available to improve HHcy-mediated muscle injury. Hydrogen sulfide $\left(\mathrm{H}_{2} \mathrm{~S}\right)$ is an antioxidant compound, and patients with CBS mutation do not produce $\mathrm{H}_{2} \mathrm{~S}$. In this study, we hypothesized that $\mathrm{H}_{2} \mathrm{~S}$ mitigates $\mathrm{HH}$ cy-induced redox imbalance/ER stress during skeletal muscle atrophy via JNK phosphorylation. We used $\mathrm{CBS}^{+/-}$mice to study atrophy via JN pl HHcy-mediated muscle atrophy, and treated them with sodium hydrogen sulfide (NaHS; an $\mathrm{H}_{2} \mathrm{~S}$ donor). Proteins and mRNAs were examined by Western blots and quantitative PCR. Proinflammatory cytokines were also measured. Muscle mass and strength were studied via fatigue susceptibility test. Our data revealed that HHcy was detrimental to skeletal mass, particularly gastrocnemius and quadriceps muscle weight. We noticed that oxidative stress was reversed by NaHS in homocysteine (Hcy)-treated C2C12 cells. Interestingly, ER stress markers (GRP78, ATF6, pIRE1 $\alpha$, and pJNK) were elevated in vivo and in vitro, and NaHS mitigated these effects. Additionally, we observed that JNK phosphorylation was upregulated in $\mathrm{C} 2 \mathrm{C} 12$ afte Hcy treatment, but NaHS could not reduce this effect. Furthermore, inflammatory cytokines IL-6 and TNF- $\alpha$ were higher in plasma from CBS as compared with wild-type mice. FOXO1-mediated Atroginand MuRF-1 upregulation were attenuated by NaHS. Functiona studies revealed that NaHS administration improved muscle fatigability in $\mathrm{CBS}^{+/-}$mice. In conclusion, our work provides evidence that $\mathrm{NaHS}$ is beneficial in mitigating HHcy-mediated skeletal injury incited by oxidative/ER stress responses.

cellular stress; cystathionine- $\beta$-synthase; inflammation; muscle atrophy; reactive oxygen species

\section{INTRODUCTION}

Homocysteine (Hcy) is a sulfur-containing non-proteinogenic amino acid that is generated during methionine metabo-

Address for reprint requests and other correspondence: A. Majumder, Dep of Biochemistry and Molecular Genetics, Univ. of Louisville School of Medicine, Louisville, KY 40202 (e-mail: avisek.majumder@louisville.edu). lism via the methionine cycle (57). In healthy subjects, synthesis and elimination of Hcy are balanced; however, if Hcy metabolism is disturbed, then its plasma levels are elevated, leading to hyperhomocysteinemia (HHcy) $(13,53,64,65,73)$. Children born with HHcy due to cystathionine- $\beta$-synthase (CBS) deficiency die shortly after birth, but children heterozygous for CBS mutation (CBS+/-) can survive $(22,65,66)$. How HHcy triggers such pathological effects in skeletal muscle are not fully understood.

Previous studies revealed that Hcy contains an - $\mathrm{SH}$ group like thiols (RSH), which can undergo oxidation to form a disulfide (RSSR) even at physiological $\mathrm{pH}$ in the presence of metal catalysts and molecular oxygen $\left[\mathrm{O}_{2}{ }^{\circ}\right](18)$. Further, Hcy can also produce hydrogen peroxide $\left(\mathrm{H}_{2} \mathrm{O}_{2}\right.$, a pro-oxidant molecule) during metal-catalyzed oxidation step and peroxynitrite $\left(\mathrm{ONOO}^{-}\right.$, a powerful oxidant $)$in the presence of nitric oxide (NO) and superoxide anion $\left(\mathrm{O}_{2}{ }^{-}\right)$(29). Although these phenomena have been studied in multiple tissue types, whether HHcy exerts its detrimental effects on muscle through these mechanisms is not yet elucidated. Oxidative stress has been implicated in many diseases associated with protein misfolding $(36,39,62)$. Likewise, studies have also reported that HHcy could induce endoplasmic reticulum (ER) stress in hepatocytes as well as in vascular endothelial and aortic smooth muscle cells, but the cellular pathways that are involved in these stress-related conditions are not adequately studied $(69,76)$. It is well known that after translation the protein folding occurs inside the ER, but during stress conditions misfolded proteins can accumulate inside ER lumen, inducing the unfolded protein response (UPR) (42). In mammals, there are three branches of UPR: inositol-requiring enzyme-1 (IRE1), PRKRlike ER kinase (PERK), and activating transcription factor-6 (ATF6) $(23,37,50)$. During severe ER stress conditions, activated IRE1 $\alpha$ recruits TNF receptor-associated factor-2 (TRAF2) and apoptosis signal-regulating kinase-1 (ASK1), which further activate c-Jun N-terminal kinase (JNK) $(6,26$, 52). Activation of JNK phosphorylates c-Jun at Ser63 and 73 residues in $\mathrm{NH}_{2}$-terminal $(7,10)$. JNK along with c-Jun makes up the activator protein-1 (AP-1) transcription factor, which regulates the expression of several proinflammatory genes $(16$, 21 ). JNK also regulates maturation and activity of $\mathrm{T}$ cells in addition to the synthesis of proinflammatory cytokines, such as interleukin-2 (IL-2), IL-6, and TNF- $\alpha(14,40,72)$. Whether 


\title{
Physiological Reports
}

ORIGINAL RESEARCH

Physiological Reports ISSN 2051-817X

\section{Hydrogen sulfide improves postischemic neoangiogenesis in the hind limb of cystathionine- $\beta$-synthase mutant mice via PPAR- $\gamma /$ VEGF axis}

\author{
Avisek Majumder ${ }^{1,2}$, Mahavir Singh ${ }^{1}$, Akash K. George ${ }^{1}$, Jyotirmaya Behera ${ }^{1}$, Neetu Tyagi ${ }^{1}$ \\ \& Suresh C. Tyagi ${ }^{1}$ \\ 1 Department of Physiology, University of Louisville School of Medicine, Louisville, Kentucky 40202, USA \\ 2 Department of Biochemistry and Molecular Genetics, University of Louisville School of Medicine, Louisville, Kentucky 40202, USA
}

Keywords

Angiogenesis, hydrogen sulfide, stress response.

\section{Correspondence}

Avisek Majumder or Mahavir Singh, Department of Biochemistry and Molecular Genetics, University of Louisville School of Medicine, Louisville, KY 40202, USA. Tel: $502-852-4425$ Fax: 502-852-6239 E-mails: avisek.majumder@louisville.edu mahavir.singh@louisville.ed

Funding Information

The work was supported by grants from the National Institute of Health (Heart, Lung, and Blood Institute; No. HL-74815, HL-107640) and the Institute of Neurological Disorders and Stroke (No. NS-084823).

Received: 5 May 2018; Revised: 10 August 2018; Accepted: 13 August 2018

doi: $10.14814 /$ phy2.13858

Physiol Rep, 6 (17), 2018, e13858, https://doi.org/10.14814/phy2.13858

\begin{abstract}
Neoangiogenesis is a fundamental process which helps to meet energy requirements, tissue growth, and wound healing. Although previous studies showed that Peroxisome proliferator-activated receptor (PPAR- $\gamma$ ) regulates neoangiogenesis via upregulation of vascular endothelial growth factor (VEGF), and both VEGF and PPAR- $\gamma$ expressions were inhibited during hyperhomocysteinemic (HHcy), whether these two processes could trigger pathological effects in skeletal muscle via compromising neoangiogenesis has not been studied yet. Unfortunately, there are no treatment options available to date for ameliorating HHcy-mediated neoangiogenic defects. Hydrogen sulfide $\left(\mathrm{H}_{2} \mathrm{~S}\right)$ is a novel gasotransmitter that can induce PPAR- $\gamma$ levels. However, patients with cystathionine- $\beta$-synthase (CBS) mutation(s) cannot produce a sufficient amount of $\mathrm{H}_{2} \mathrm{~S}$. We hypothesized that exogenous supplementation of $\mathrm{H}_{2} \mathrm{~S}$ might improve $\mathrm{HHcy}$-mediated poor neoangiogenesis via the PPAR- $\gamma /$ VEGF axis. To examine this, we created a hind limb femoral artery ligation (FAL) in $\mathrm{CBS}^{+/-}$mouse model and treated them with GYY4137 (a long-acting $\mathrm{H}_{2} \mathrm{~S}$ donor compound) for 21 days. To evaluate neoangiogenesis, we used barium sulfate angiography and laser Doppler blood flow measurements in the ischemic hind limbs of experimental mice post-FAL to assess blood flow. Proteins and mRNAs levels were studied by Western blots and qPCR analyses. HIF1- $\alpha$, VEGF, PPAR- $\gamma$ and p-eNOS expressions were attenuated in skeletal muscle of $\mathrm{CBS}^{+/-}$mice after 21 days of FAL in comparison to wild-type (WT) mice, that were improved via GYY4137 treatment. We also found that the collateral vessel density and blood flow were significantly reduced in postFAL $\mathrm{CBS}^{+/-}$mice compared to WT mice and these effects were ameliorated by GYY4137. Moreover, we found that plasma nitrite levels were decreased in post-FAL $\mathrm{CBS}^{+/-}$mice compared to WT mice, which were mitigated by GYY4137 supplementation. These results suggest that HHcy can inhibit neoangiogenesis via antagonizing the angiogenic signal pathways encompassing PPAR- $\gamma /$ VEGF axis and that GYY4137 could serve as a potential therapeutic to alleviate the harmful metabolic effects of HHcy conditions.
\end{abstract}

\section{Introduction}

Homocysteine (Hcy) has been studied extensively for over 30 years for its unique involvement in an increasing number of human diseases (Hankey and Eikelboom 1999;
Narayanan et al. 2014; Stipanuk and Ueki 2011). The Hcy level is controlled by two major processes: around $50 \%$ of Hcy enters the transsulfuration pathway to produce cysteine, and the other half is remethylated back to methionine (Met) via the folate 1-carbon cycle (Cascella et al 


\title{
Rem ote ischemic conditioning as a cytoprotective strategy in vasculopathies during hyperhom ocysteinemia: An emerging research perspective
}

\author{
Avisek Majumder ${ }^{1,3}$ (1) | Mahavir Singh ${ }^{1,2} \mid$ Akash K. George ${ }^{1,2} \mid$ \\ Rubens Petit Homme ${ }^{1,2} \mid$ Anwesha Laha ${ }^{1}$ Suresh C. Tyagi ${ }^{1}$
}

${ }^{1}$ Department of Physiology, School of Medicine, University of Louisville

Louisville, Kentucky

${ }^{2}$ Eye and Vision Science Laboratory University of Louisville, Louisville, Kentucky

${ }^{3}$ Department of Biochemistry and Molecular Genetics, School of Medicine University of Louisville, Louisville, Kentucky

Corr respondence Avisek Majumder and Mahavir Singh, Department of Physiology, School of

Medicine, University of Louisville Louisville 40202, KY.

Email: avisek.majumder@louisville.edu; mahavir.singh@louisville.edu

Funding information National Institute of Health, Grant/Award Numbers: HL-74815, AR071789, and HL-139047

\section{Abstract}

Higher levels of nonprotein amino acid homocysteine (Hcy), that is, hyperhomocysteinemia ( $\mathrm{HHcy}$ ) ( $-5 \%$ of general population) has been associated with severe vasculopathies in different organs; however, precise molecular mechanism(s) as to how HHcy plays havoc with body's vascular networks are largely unknown. Interventional modalities have not proven beneficial to counter multifactorial HHcy's effects on the vascular system. An ancient Indian form of exercise called 'yoga' causes transient ischemia as a result of various body postures however the cellular mechanisms are not clear. We discuss a novel perspective wherein we argue that application of remote ischemic conditioning (RIC) could, in fact, deliver anticipated results to patients who are suffering from chronic vascular dysfunction due to HHcy. RIC is the mechanistic phenomenon whereby brief episodes of ischemia-reperfusion events are applied to distant tissues/organs; that could potentially offer a powerful tool in mitigating chronic lethal ischemia in target organs during HHcy condition via simultaneous reduction of inflammation, oxidative and endoplasmic reticulum stress, extracellular matrix remodeling, fibrosis, and angiogenesis. We opine that during ischemic conditioning our organs cross talk by releasing cellular messengers in the form of exosomes containing messenger RNAs, circular RNAs, anti-pyroptotic factors, protective cytokines like musclin, transcription factors, small molecules, anti-inflammatory, antiapoptotic factors, antioxidants, and vasoactive gases. All these could help mobilize the bone marrow-derived stem cells (having tissue healing properties) to target organs. In that context, we argue that RIC could certainly play a savior's role in an unfortunate ischemic or adverse event in people who have higher levels of the circulating $\mathrm{Hcy}$ in their systems.

KEYWORDS

cardio protection, inflammation, ischemia, ischemic preconditioning, oxidative stress 
www.impactjournals.com/oncotarget/ Oncotarget, 2017, Vol. 8, (No. 60), pp: 102739-102758

Review

\title{
Post-menopausal breast cancer: from estrogen to androgen receptor
}

\author{
Avisek Majumder ${ }^{1,2}$, Mahavir Singh ${ }^{2}$ and Suresh C. Tyagi ${ }^{2}$ \\ ${ }^{1}$ Department of Biochemistry and Molecular Genetics, University of Louisville School of Medicine, Louisville 40202, Kentucky, \\ USA \\ ${ }^{2}$ Department of Physiology, University of Louisville School of Medicine, Louisville 40202, Kentucky, USA \\ Correspondence to: Avisek Majumder, email: avisek.majumder@lovisville.edu \\ Mahavir Singh, email: mahavir.singh@lovisville.edu \\ Keywords: breast cancer; androgen receptor; estrogen receptor; metastasis; post-menopausal women \\ Received: July 06, $2017 \quad$ Accepted: September 29, $2017 \quad$ Published: October 27, 2017 \\ Copyright: Majumder et al. This is an open-access article distributed under the terms of the Creative Commons Attribution License \\ 3.0 ( $C$ C BY 3.0), which permits unrestricted use, distribution, and reproduction in any medium, provided the original author and \\ source are credited.
}

ABSTRACT

In the United States, breast cancer is the second leading cause of death among women, and even though different therapies can treat primary breast tumors, most breast cancer-related deaths ( $>95 \%$ ) occur due to metastasis. A majority ( $70 \%)$ of breast tumors are found to express estrogen receptor, and a significant portion ( $~ 90 \%)$ of ER-positive $\left(E R^{+}\right.$) breast tumors are also androgen receptor-positive $\left(A R^{+}\right)$. Although ER is known to promote tumorigenesis, the role and underlying mechanism(s) of AR in these closely knit processes remain controversial. Endocrine therapies are the most commonly used treatment for patients with $E R^{+}$breast tumors; but, $\sim 30 \%-50 \%$ of initially responsive patients develop resistance to these therapies. Whereas $\mathbf{7 0} \%-\mathbf{9 0} \%$ of all breast tumors are $\mathbf{A R}^{+}$and $\mathbf{A R}$ overexpression is correlated with endocrine resistance, but the precise molecular mechanism(s) for this association is yet to be studied. Multiple mechanisms have been proposed to show AR and $E R$ interactions, which indicate that $A R$ may preferentially regulate expression of a subset of ER-responsive genes and that may be responsible for breast cancer and its progression in affected patients. On the other hand, most of the $E R^{+}$breast tumors found in post-menopausal women ( $~ 80 \%)$; and they have very low $17 \beta$-estradiol and high androgen levels, but how these hormonal changes make someone more prone to cancer phenotype has long been a disputed issue. In this study, we have discussed multiple molecular mechanisms that we believe are central to the understanding of the overall contributions of $A R$ in breast cancer and its metastasis in post-menopausal women.

\section{INTRODUCTION}

In the United States, breast cancer is the second leading cause of death among women (40,610 deaths and 252,710 new cancer cases are estimated in 2017) [1]. There are different therapies (surgery, radiotherapy, chemotherapy, endocrine therapies, or combination of these therapies) available which can treat primary tumor; however, most breast cancer-related deaths occur due to distance organ metastasis (mainly lung, brain and liver). Whereas, most epidemiological studies showed inconsistent finding in a correlation between serum androgen levels and breast cancer risk; but whether this association is consistent in post-menopausal women with breast cancers (who have high androgens levels and low $17 \beta$-estradiol) is not understood [2-11]. Previous studies have implicated a possible role for AR in breast cancers [12-14], and AR is found to be predominantly expressed in in-situ, invasive and metastatic breast cancers ( 90\% of primary tumors and $75 \%$ of metastases) [15-18]; however, the precise molecular mechanism(s) for AR's contribution to breast cancer is largely unknown. Hence, a 


\title{
Hypermethylation: Causes and Consequences in Skeletal Muscle Myopathy
}

\author{
Avisek Majumder, ${ }^{1,2}$ Jyotirmaya Behera, ${ }^{2}$ Nevena Jeremic, ${ }^{2}$ and Suresh C. Tyagi ${ }^{1,2 *}$ \\ ${ }^{1}$ Department of Biochemistry and Molecular Genetics, School of Medicine, University of Louisville, Louisville, \\ Kentucky 40202 \\ ${ }^{2}$ Department of Physiology, School of Medicine, University of Louisville, Louisville, Kentucky 40202
}

\section{ABSTRACT}

A detrimental consequence of hypermethylation is hyperhomocysteinemia (HHcy), that causes oxidative stress, inflammation, and matrix degradation, which leads to multi-pathology in different organs. Although, it is well known that hypermethylation leads to overall gene silencing and hypomethylation leads to overall gene activation, the role of such process in skeletal muscle dysfunction during HHcy condition is unclear. In this study, we emphasized the multiple mechanisms including epigenetic alteration by which HHcy causes skeletal muscle myopathy. This review also highlights possible role of methylation, histone modification, and RNA interference in skeletal muscle dysfunction during HHcy condition and potential therapeutic molecules, putative challenges, and methodologies to deal with HHcy mediated skeletal muscle dysfunction. We also highlighted that B vitamins (mainly B12 and B6), with folic acid supplementation, could be useful as an adjuvant therapy to reverse these consequences associated with this HHcy conditions in skeletal muscle. However, we would recommend to further study involving long-term trials could help to assess efficacy of the use of these therapeutic agents. J. Cell. Biochem. 9999: 1-10, $2017 . \quad$ () 2017 Wiley Periodicals, Inc.

\section{KEY WORDS: HYPERMETHYLATION; HOMOCYSTEINE; OXIDATIVE STRESS; MUSCLE DYSFUNCTION; ECM REMODELING}

$\mathrm{H}$

yperhomocysteinemia (HHcy/HHCY) is a complex metabolic multifactorial disorder with defects in Hcy (thiol-containing amino acid) metabolism with high prevalence in the general population $(\sim 5 \%)$ [Brustolin et al., 2010]. HHcy is typically caused either by genetic defects in the enzymes involved in Hcy metabolism (e.g., $677 \mathrm{C}>\mathrm{T}$ and $1298 \mathrm{~A}>\mathrm{C}$ polymorphism in methylene tetrahydrofolate reductase MTHFR gene) or by nutritional deficiencies in vitamin B12, B6, and folate [Brustolin et al., 2010; Picker and Levy, 2014]. There are also several factors such as age, sex, physical activity, alcohol intake, certain medications, and different disease conditions that can modulate the Hcy level in blood [Ames et al., 2002; Van Guldener, 2006; Signorello et al., 2007; Neuman et al., 2013; Choi et al., 2015]. Depending upon severity of this HHcy conditions it can be classified as follows: (1) moderate HHcy: 15 and $30 \mu \mathrm{mol} / \mathrm{L}$; (2) intermediate HHcy: 30-100 $\mu \mathrm{mol} / \mathrm{L}$; and (3) severe HHcy: $>100 \mu \mathrm{mol} / \mathrm{L}$ [Tiahou et al., 2009]. Hcy is produced during the methionine (MET) cycle, where dietary MET is first converts to S-adenosylmethionine (SAM), then SAM converts to S-adenosylhomocysteine(SAH), which then, finally converts to Hcy [Veeranki and Tyagi, 2013]. The plasma level of Hcy is controlled by two processes: either Hcy is degraded to cysteine via transsulfuration reaction using cystathionine beta synthase (CBS), cystathionine $\gamma$-lyase (CSE) enzyme, and B6 as a cofactor or Hcy can be re-methylated to MET by MET-synthase using vitamin B12 as a cofactor and MTHF as the methyl donor through folate cycle [Veeranki and Tyagi, 2013]. In normal conditions, the synthesis and elimination of Hcy are in balance $(\sim 50 \%$ being re-methylated and $\sim 50 \%$ eliminated) [Cascella et al., 2015], however, in diseased state the rate of cellular Hcy synthesis is faster than the elimination, which leads to excess release of Hcy into the blood circulation, and this condition is known as HHcy (Fig. 1) [Cascella et al., 2015].

HHcy is well accepted risk factor for vascular diseases [Markand et al., 2015], however, several studies also reported that HHcy conditions leads to skeletal muscle weakness and functional impairment [Veeranki and Tyagi, 2013; Picker and Levy, 2014]. Children born with severe homocystinuria due to CBS deficiency exhibit poor body weight, skeletal muscle myopathy, and die in teenage [Picker and Levy, 2014], whereas HHcy condition displays decreased bodyweight and skeletal muscle mass, which ultimately leads to muscle myopathy [Veeranki and Tyagi, 2013]. Results from our laboratory in this field, previously, showed that the expression of CBS and CSE were very less in skeletal muscles, which increases the

Grant sponsor: National Institutes of Health grants; Grant number: HL-74185.

"Correspondence to: Suresh C. Tyagi, PhD, Department of Physiology, Health Sciences Center, University of Louisville, RM-1215, Louisville, KY 40202. E-mail: suresh.tyagi@louisville.edu

Manuscript Received: 13 December 2016; Manuscript Accepted: 14 December 2016

Accepted manuscript online in Wiley Online Library (wileyonlinelibrary.com): 16 December 2016

DOI 10.1002/jcb.25841 • (ㅇ 2017 Wiley Periodicals, Inc. 

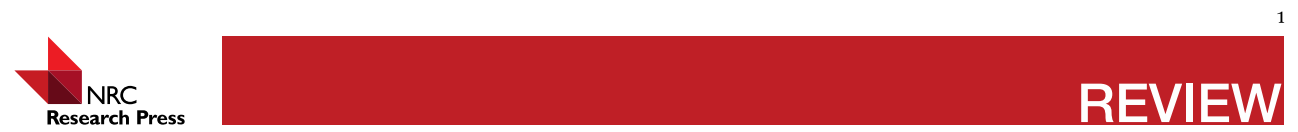

\title{
Connecting homocysteine and obesity through pyroptosis, gut microbiome, epigenetics, peroxisome proliferator-activated receptor $\gamma$, and zinc finger protein $407^{1}$
}

Anwesha Laha, Avisek Majumdar, Mahavir Singh, and Suresh C. Tyagi

\begin{abstract}
Although homocysteine (Hcy), a part of the epigenome, contributes to cell death by pyroptosis and decreases peroxisome proliferator-activated receptor $\gamma$ (PPAR $\gamma$ ) levels, the mechanisms are unclear. Hcy is found in high concentrations in the sera of obese individuals, which can elicit an immune response as well by hypermethylating $C P G$ islands of specific gene promoters, a marker of epigenetics. Hcy has also been established to chelate divalent metal ions like $\mathrm{Cu}^{2+}$ and $\mathrm{Zn}^{2+}$, but this role of Hcy has not been established in relationship with obesity. It has been known for a while that PPAR $\gamma$ dysregulation results in various metabolic disorders including glucose and lipid metabolism. Recently, zinc finger protein 407 (Zfp407) is reported to regulate PPAR $\gamma$ target gene expression without affecting PPAR $\gamma$ transcript and protein levels by synergistically working with PPAR $\gamma$. However, the mechanism(s) of this synergy, as well as other factors contributing to or inhibiting this synergism, have not been proven. This review suggests that Hcy contributes to pyroptosis, changes gut microbiome, and alters PPAR $\gamma$-dependent mechanism(s) via Zfp407-mediated upregulated adipogenesis and misbalanced fatty acid metabolism, which can predispose to obesity and, consequently, obesity-related metabolic disorders.

Key words: PGC-1 $\alpha$, zinc finger protein, DNA methylation, lipopolysaccharide, fatty acid metabolism, type 2 diabetes, insulin.

Résumé : Bien que l'on sache que l'homocystéine (Hcy), une partie de l'épigénome, contribue à la mort cellulaire par la pyroptose et qu'elle entraîne une diminution des taux de récepteurs $\gamma$ activés par les proliférateurs de peroxysomes (ou $\operatorname{PPAR} \gamma$ pour " peroxisome proliferator-activated receptor $\gamma$ »), les modes d'action demeurent à éclaircir. On trouve de l'Hcy à concentration élevée dans le sérum de personnes obèses, ce qui peut aussi provoquer une réaction immunitairepa l'hyperméthylation des îlots $\mathrm{CpG}$ de promoteurs de gènes spécifiques, un marqueur de l'épigénétique. On a aussi établi que l'Hcy entre en chélation avec des ions métalliques divalents comme $\mathrm{Cu}^{2+}$ et $\mathrm{Zn}^{2+}$, mais ce rôle de l'Hcy n'a pas été établi en relation avec l'obésité. On sait depuis un certain temps que la dérégulation des PPAR $\gamma$ entraîne diverses maladies d'ordre métabolique mettant en jeu le métabolisme du glucose et des lipides. On a rapporté récemment que la protéine à doigt de zinc 407 (Zfp407) participe à la régulation de l'expression de gènes cibles des PPAR $\gamma$ sans modifier les taux de transcrits et de protéines des PPAR $\gamma$ en agissant en synergie avec les PPAR $\gamma$. Cependant, le ou les modes d'action de cette synergie, ainsi que d'autres facteurs contribuant à cette en synergie avec les PPAR $\gamma$. Cependant, le ou les modes d'action de cette synergie, ainsi que d'autres facteurs contribuant à cette
synergie ou à son inhibition ne sont pas appuyés par des données probantes. Cet article de synthèse laisse entrevoir que l'Hcy participe à la pyroptose et entraîne des modifications du microbiome entérique et d'éventuellement plusieurs modes d'action dépendants des PPAR $\gamma$ par l'intermédiaire de la régulation à la hausse de l'adipogenèse et d'un déséquilibre de la régulation du métabolisme des acides gras, tous deux médiés par la Zfp407, ce qui peut prédisposer à l'obésité et consécutivement aux maladies d'ordre métabolique liées à l'obésité. [Traduit par la Rédaction]
\end{abstract}

Mots-clés : PGC-1 $\alpha$, protéine à doigt de zinc, méthylation de l'ADN, lipopolysaccharide, métabolisme des acides gras, diabète de type 2 , insuline.

Homocysteine (Hcy) and obesity

Obesity is one of the leading causes of health complications like type 2 diabetes, stroke, hypertension, cardiovascular diseases, and even some cancers. As of 2014, the World Health Organization even some cancers. As of 2014, the World Health Organization stated that 1 in 5 individuals in the United States died due to
obesity-related health disorders. Per Center for Disease Control $20 \%$ of the population in every state in the United States is obese (World Health Organization, Center for Disease Control). Obesity is responsible for causing the insulin resistance leading to dysregulated lipid metabolism by reducing adipogenesis and increasing free fatty acids in the blood (Harmancey and Smih 2007: Kim et al. 2000; Odegaard and Chawla 2013).
Hcy is a non-protein amino acid that is generated during methionine metabolism. The fate of Hcy is either remethylation to methionine or conversion to cysteine through the transsulfuration pathway. Folic acid works as the cofactor essential for remethylation of Hcy to methionine (Barshop 2012). The 2 essential enzymes required for Hcy to cysteine transsulfuration are cystathionine $\beta$-synthase and cystathionine $\gamma$-lyase (Fig. 1). In the rate-limiting step, cystathionine $\beta$-synthase catalyzes the synthesis of the intermediate, cystathionine from Hcy, and cystathionine $\gamma$-lyase converts cystathionine to cysteine. Hyperhomocysteinemia (HHcy) is a medical condition that is characterized by the $\mathrm{Hcy}$ level that is more than $15 \mu \mathrm{mol} / \mathrm{L} \mathrm{Hcy}$ in the blood due to the

Received 19 January 2018. Accepted 1 May 2018.

A. Laha, A. Majumdar, M. Singh, and S.C. Tyagi. Department of Physiology, University of Louisville, Louisville, KY 40202, USA

Corresponding authors: Anwesha Laha (email: anwesha.laha@louisville.edu) and Mahavir Singh (email: mahavir.singh@louisville.edu).

'This Review is part of a Special Issue of selected papers from the 4th European Section Meeting of the International Academy of Cardiovascular Sciences held in Pécs, Hungary, on 28-30 September 2017.

Copyright remains with the author(s) or their institution(s). Permission for reuse (free in most cases) can be obtained from RightsLink. 


\section{Remodeling of Retinal Architecture in Diabetic Retinopathy: Disruption of Ocular Physiology and Visual Functions by Inflammatory Gene Products and Pyroptosis}

Rubens $P$. Homme ${ }^{1,2+}$, Mahavir Singh ${ }^{1,2 * t}$, Avisek Majumder ${ }^{1,3}$, Akash K. George ${ }^{1,2}$ Kavya Nair ${ }^{1,2}$, Harpal S. Sandhu ${ }^{4,5}$, Neetu Tyagi ${ }^{2}$, David Lominadze ${ }^{2}$ and Suresh C Tyagi ${ }^{2}$

'Eye and Vision Science Laboratory, Department of Physiology, University of Louisville School of Medicine, Louisville, KY, United States, ${ }^{2}$ Department of Physiology, University of Louisville School of Medicine, Louisville, KY, United States, ${ }^{3}$ Department of Biochemistry and Molecular Genetics, University of Louisville School of Medicine, Louisville, KY,

OPEN ACCESS KY, United St, Department of Ophthalmology and Visual Sciences, University of Louisville School of Medicine, Louisville

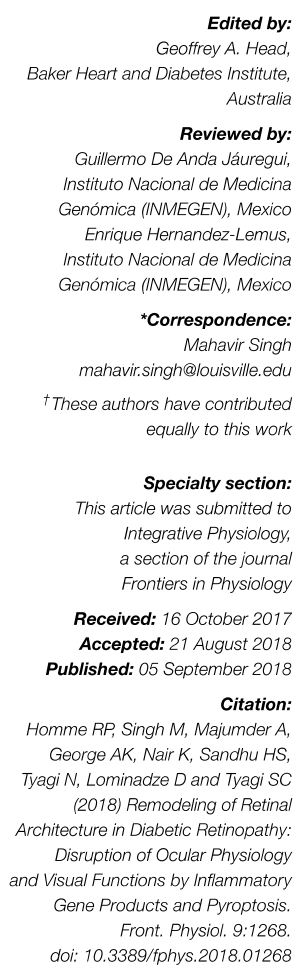

Edited by: Geoffrey A. Head, Baker Heart and Diabetes Institute Australia

Reviewed by: Guillermo De Anda Jáuregui, Instituto Nacional de Medicina Genómica (INMEGEN), Mexico

Enrique Hernandez-Lemus, Instituto Nacional de Medicina Genómica (INMEGEN), Mexico

${ }^{*}$ Correspondence: Mahavir Singh mahavir.singh@/ouisville.edu tThese authors have contributed equally to this work

Specialty section: This article was submitted to Integrative Physiology, a section of the journa Frontiers in Physiology

Received: 16 October 2017 Accepted: 21 August 2018 Published: 05 September 2018

Citation:

Homme RP, Singh M, Majumder A, George AK, Nair K, Sandhu HS, Tyagi N, Lominadze D and Tyagi SC (2018) Remodeling of Retina Architecture in Diabetic Retinopathy: Disruption of Ocular Physiology and Visual Functions by Inflammatory Gene Products and Pyroptosis. Front Physiol 9:1268. doi: 10.3389/fphys.2018.01268

Diabetic patients suffer from a host of physiological abnormalities beyond just those of glucose metabolism. These abnormalities often lead to systemic inflammation via modulation of several inflammation-related genes, their respective gene products, homocysteine metabolism, and pyroptosis. The very nature of this homeostatic disruption re-sets the overall physiology of diabetics via upregulation of immune responses, enhanced retinal neovascularization, upregulation of epigenetic events, and disturbances in cells' redox regulatory system. This altered pathophysiological milieu can lead to the development of diabetic retinopathy (DR), a debilitating vision-threatening eye condition with microvascular complications. DR is the most prevalent cause of irreversible blindness in the working-age adults throughout the world as it can lead to severe structural and functional remodeling of the retina, decreasing vision and thus diminishing the quality of life. In this manuscript, we attempt to summarize recent developments and new insights to explore the very nature of this intertwined crosstalk between components of the immune system and their metabolic orchestrations to elucidate the pathophysiology of DR. Understanding the multifaceted nature of the cellular and molecular factors that are involved in DR could reveal new targets for effective diagnostics, therapeutics, prognostics, preventive tools, and finally strategies to combat the development and progression of DR in susceptible subjects.

Keywords: chemokines, cytokines, diabetic retinopathy, epigenomics, homocysteine, inflammation, pyroptosis, signaling pathways Abbreviations: AR, aldose reductase; $B R B$, blood-retinal barrier; $C D C$, center for disease control; CVDs, cardiovascular diseases, DM, diabetes mellitus, DME, diabetic macular edema; DR, diabetic retinopathy; ECM, extracellular matri HbA1C, hemoglobin AIC, Hey, homocysteine, HNE, 4-hydroxynonenal; MI, myocardial infarction; MMPs, matrix metalloproteinases; $\mathrm{NV}$, heovascularzaton, $\mathrm{PDR}$, prolierative diabetic rethopathy; PRP, pan-retinal photocoagulation; PUFAs, poly unsaturated fatty acids, RPE, retinal pigment epithelium, TID, type I diabetes, T2D, type II diabetes; TGF- $\beta$, transforming growth factor $-\beta$; TRD, tractional retinal detachment; VEGF, vascular endothelial growth factor; WHO, Worl Health Organization 
Circular RNAs profiling in the cystathionine- $\beta$-synthase mutant mouse reveals novel gene targets for hyperhomocysteinemia induced ocular disorders

Mahavir Singh ${ }^{\mathrm{a}, \mathrm{b}, *, 1}$, Akash K. George $\mathrm{e}^{\mathrm{a}, \mathrm{b}, 1}$, Rubens Petit Homme ${ }^{\mathrm{a}, \mathrm{b}}$, Avisek Majumder ${ }^{\mathrm{b}}$, Anwesha Laha ${ }^{b}$, Harpal S. Sandhu ${ }^{c, d}$, Suresh C. Tyagi ${ }^{b}$

a Eye and Vision Science Laboratory, Department of Physiology, University of Louisville School of Medicine, Louisville, KY 40202, USA

b Department of Physiology, University of Louisville School of Medicine, Louisville, KY 40202, USA

${ }^{\mathrm{c}}$ Department of Ophthalmology and Visual Sciences, University of Louisville School of Medicine, Louisville, KY 40202, USA

d Kentucky Lions Eye Center, University of Louisville School of Medicine, Louisville, KY 40202, USA

A R T I C L E I N F O

Keywords:

Keywords:
Circular RNA

Circular RNA

Epigenetics

Eye diseases

Homocysteine

Oxidative-stress

\begin{abstract}
A B S T R A C T
Cystathionine- $\beta$-synthase (CBS) gene encodes L-serine hydrolyase which catalyzes $\beta$-reaction to condense serine with homocysteine (Hcy) by pyridoxal-5'-phosphate helps to form cystathionine which in turn is converted to with homocysteine (Hcy) by pyridoxal-5'-phosphate helps to form cystathionine which in turn is converted to
cysteine. CBS resides at the intersection of transmethylation, transsulfuration, and remethylation pathways, thus lack of CBS fundamentally blocks Hcy degradation; an essential step in glutathione synthesis. Redox homeostasis, free-radical detoxification and one-carbon metabolism (Methionine-Hcy-Folate cycle) require CBS and its deficiency leads to hyperhomocysteinemia (HHcy) causing retinovascular thromboembolism and eye-lens dislocation along with vascular cognitive impairment and dementia. HHcy results in retinovascular, coronary, cerebral and peripheral vessels' dysfunction and how it causes metabolic dysregulation predisposing patients to serious eye conditions remains unknown. HHcy orchestrates inflommation and redox imbalance via epigenetic serious eye conditions remains innown. remodeling leading to neurovascular pathologies. Although circular RNAs (circRNAs) are dominant players
regulating their parental genes' expression dynamics, their importance in ocular biology has not been appreregulating their parental genes' expression dynamics, their importance in ocular biology has not been appre-
ciated. Progress in gene-centered analytics via improved microarray and bioinformatics are enabling dissection of genomic pathways however there is an acute under-representation of circular RNAs in ocular disorders. This study undertook circRNAs' analysis in the eyes of CBS deficient mice identifying a pool of 12532 circRNAs, 74 exhibited differential expression profile, $\_27 \%$ were down-regulated while most were up-regulated $(\sim 73 \%)$ Findings also revealed several microRNAs that are specific to each circRNA suggesting their roles in HHcy induced ocular disorders. Further analysis of circRNAs helped identify novel parental genes that seem to influence certain eye disease phenotypes.
\end{abstract}

\section{Introduction}

Our current understanding of circular RNAs (circRNAs) mediated cellular gene expression phenomena that are responsible for a multitude of molecular pathways is still naïve partly because their putative roles in the context of health and disease are under intense investigations. In comparison to other tissues, circRNAs appears to be highly enriched in the central nervous system (CNS) (Rybak-Wolf et al., 2015; You et al., 2015). Regarding their roles in eyes, the limited availability of literature about their abundance makes it difficult to study their structural and functional aspects pertaining to important ocular disorders (Han et al., 2017; Luo et al., 2017; Zhang et al, 2017). CircRNAs are considered as new class of non-coding entities but endowed with significant regulatory abilities through their protein or miRNA "sponging" abilities thereby effectively controlling mRNA transcription and translation machineries (Hansen et al., 2013; Memczak et al., 2013).

In the past, many microRNAs (miRNAs) have been identified and subsequently characterized inside cells and tissues of the eye, however; the expression levels of the circRNAs that are responsible for regulating them have not been fully studied. Although public databases provide some information about circRNAs abundance in other tissues of mouse

\footnotetext{
* Corresponding author. Eye and Vision Science Laboratory, Department of Physiology, University of Louisville School of Medicine, Louisville, KY 40202, USA

E-mail addresses: mahavir.singh@louisville.edu, gene2genetics@gmail.com (M. Singh).

${ }^{1}$ Denotes equal contribution

https://doi.org/10.1016/j.exer.2018.05.026

Received 28 March 2018; Received in revised form 9 May 2018; Accepted 23 May 2018

Available online 25 May 2018

0014-4835/@ 2018 Elsevier Ltd. All rights reserved.
} 


\title{
A hypothesis for treating inflammation and oxidative stress with hydrogen sulfide during age-related macular degeneration
}

\author{
Akash K George ${ }^{1,2}$, Mahavir Singh ${ }^{1,2}$, Rubens Petit Homme ${ }^{1,2}$, Avisek Majumder ${ }^{2,3}$, Harpal S Sandhu ${ }^{4,5}$, \\ Suresh C Tyagi ${ }^{2}$
}

${ }^{1}$ Eye and Vision Science Laboratory, Department of Physiology, University of Louisville School of Medicine, Louisville, Kentucky 40202, USA

${ }^{2}$ Department of Physiology, University of Louisville School of Medicine, Louisville, Kentucky 40202, USA

${ }^{3}$ Department of Biochemistry and Molecular Genetics, University of Louisville School of Medicine, Louisville, Kentucky 40202, USA

${ }^{4}$ Department of Ophthalmology and Visual Sciences, University of Louisville School of Medicine, Louisville, Kentucky 40202, USA

${ }^{5}$ Kentucky Lions Eye Center, University of Louisville School of Medicine, Louisville, Kentucky 40202, USA

Co-first authors: Akash K George and Mahavir Singh

Correspondence to: Mahavir Singh. Eye and Vision Science Laboratory, Department of Physiology, University of Louisville School of Medicine, Louisville, Kentucky 40202, USA. mahavir.singh@louisville.edu; gene2genetics@gmail.com Received: 2017-11-23 Accepted: 2018-03-12

\footnotetext{
Abstract

- Age-related macular degeneration (AMD) is a leading cause of blindness and is becoming a global crisis since affected people will increase to 288 million by 2040 . Genetics, age, diabetes, gender, obesity, hypertension, race, hyperopia, iris-color, smoking, sun-light and pyroptosis have varying roles in AMD, but oxidative stress-induced inflammation remains a significant driver of pathobiology. Eye is a unique organ as it contains a remarkable oxygengradient that generates reactive oxygen species (ROS) which upregulates inflammatory pathways. ROS becomes a source of functional and morphological impairments in retinal pigment epithelium (RPE), endothelial cells and retinal ganglion cells. Reports demonstrated that hydrogen sulfide $\left(\mathrm{H}_{2} \mathrm{~S}\right)$ acts as a signaling molecule and that it may treat ailments. Therefore, we propose a novel hypothesis that $\mathrm{H}_{2} \mathrm{~S}$ may restore homeostasis in the eyes thereby reducing damage caused by oxidative injury and inflammation. Since $\mathrm{H}_{2} \mathrm{~S}$ has been shown to be a powerful antioxidant because of its free-radicals' inhibition properties in addition to its beneficial effects in age-related
}

conditions, therefore, patients may benefit from $\mathrm{H}_{2} \mathrm{~S}$ salubrious effects not only by minimizing their oxidant and inflammatory injuries to retina but also by lowering retinal glutamate excitotoxicity.

- KEYWORDS: eye diseases; hydrogen sulfide treatment; inflammation; macula; oxidative stress; pyroptosis; retinal degeneration

DOI:10.18240/ijo.2018.05.26

Citation: George AK, Singh M, Homme RP, Majumder A, Sandhu HS, Tyagi SC. A hypothesis for treating inflammation and oxidative stress with hydrogen sulfide during age-related macular degeneration. Int J Ophthalmol 2018;11(5):881-887

\section{INTRODUCTION}

imilar to many aging diseases such as neurodegeneration, $\checkmark$ diabetes, cancer and atherosclerosis, role of ocular inflammation mediated by the disruption of redox homeostasis has been studied extensively in age-related macular degeneration (AMD) ${ }^{[1]}$. Since retina is one of the highest oxygen consuming tissues in our body, it generates significant reactive oxygen species (ROS) moieties and related radical contents (Figure 1), which makes it vulnerable to oxidative injury over time ${ }^{[2-3]}$. A large amount of oxygen resides in the choroid and as oxygen tension falls across retinal pigment epithelium (RPE) and outer retina, it creates a vast oxygen gradient towards inner segments of the eyes' photoreceptor components. Also, photoreceptors in the retina contain relatively high levels of polyunsaturated fatty acids (PUFA) in comparison to other tissues. ROS-initiated lipid peroxidation reactions also generate reactive carbonyl compounds (RCC) from these biological lipids which further adds fuel to chronic neurodegenerative conditions such retinal degeneration ${ }^{[4]}$. Due to continuous accumulation of lipofuscin, which causes photooxidative damage (lipofuscin is a product of oxidation of lipids and lipoproteins containing photo-oxidative fluorophores such as green light-emitting retinol and retinyl esters), together with other photosensitizers. Abundant light exposure and a high metabolic demand make retina a prime location for the oxidative damage (Figure 1). The non-degradable 


\title{
Expression Analysis of the Circular RNA Molecules in the Human Retinal Cells Treated with Homocysteine
}

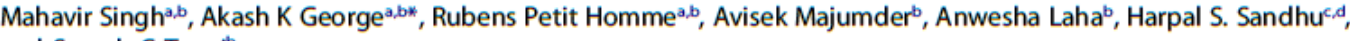 \\ and Suresh C Tyagi
}

'Eye and Vision Science Laboratory, Department of Physiology, University of Louisville School of Medicine, Louisville, Kentucky, USA; 'Department of Physiology, University of Louisville School of Medicine, Louisville, Kentucky, USA; 'Department of Ophthalmology and Visual Sciences, University of Louisville School of Medicine, Louisville, Kentucky, USA; 'Kentucky Lions Eye Center, University of Louisville School of Medicine, Louisville, Kentucky, USA

ABSTRACT

Purpose: To characterize the global profile of circular RNAs (circRNAs) and their differential expression levels in homocysteine (Hcy)-treated ARPE-19 cells, a line of human retinal pigment epithelial (RPE) cells. Materials and Methods: We treated ARPE-19 cells with and without Hcy to investigate the influence of Hcy on circRNA expression levels using dedicated human circRNA microarrays.

Results: A total of 12,233 circRNAs were identified out of them 54 were differentially expressed (17 were down-regulated, and 37 were up-regulated) with a fold change $>2.0(p<0.05)$ in Hcy-treated versus untreated cells.

Conclusions: To our knowledge, this is the first report profiling circRNAs in human RPE cells post-Hcy treatment mimicking hyperhomocysteinemic (HHcy) conditions that negatively affect retinal biology and vision. These findings are of potential clinical significance as it will help understand Hcy metabolism and $\mathrm{HHcy}$-mediated diseases and identify potential diagnostic and therapeutic targets for eye diseases that are caused by elevated Hcy concentrations.

\section{Introduction}

Retinovascular disease, comprised primarily of diabetic retinopathy (DR) and retinal vein occlusion (RVO), is a major cause of vision loss in the working age population. Its cardina features are vascular leakage, inflammation, thrombosis, and angiogenesis. ${ }^{1-5}$ Despite advances in anti-angiogenic treatment in ophthalmology, the retinovascular disease still cause significant morbidity. Recently, circRNAs have been shown to regulate RNA and protein expression in the retina. ${ }^{6}$ Circular RNAs (circRNAs) are a recently re-discovered class of endogenous RNAs that are generated from exons, introns, or both via a diverse number of cellular mechanisms. Rapid progress in high-throughput sequencing and bioinformatics has revealed that many circRNAs can modulate expression of microRNAs (miRNAs or miRs). ${ }^{7-10}$ More importantly, circRNAs fine-tune post-transcriptional expression via their interactions with miRNAs or with single or multiple RNAbinding proteins. ${ }^{8}$

In the eye, circRNAs can either promote ocular homeostasis and normal function or contribute to disease. For example, circRNA-ZNF609 can rescue vascular endothelial dysfunction in an oxygen-induced retinopathy model whereas CircHIIPK3 plays a negative role in DR. ${ }^{11,12}$ Identification of circRNAs that are expressed in retinal pigment epithelial
(RPE) cells could shed light on their role in the eye, especially under hyperhomocysteinemic (HHcy) conditions. This could also assist in finding clinical biomarkers for treatment of retinal disorders. To the best of our knowledge, no study has examined circRNAs under HHcy conditions in the human eyes. This study sought to characterize the global profile of circRNAs in RPE cells under HHcy conditions. The RPE is an essential monolayer of cells immediately deep to the neurosensory retina which provides it with nutrition, allows the diffusion of oxygen to the outer retina, and phagocytoses outer photoreceptor segments to keep these cells functional. The proper function of the RPE is also critical to the maintenance of the blood-retina barrier (BRB). An intact BRB prevents the accumulation of serous fluid under or potentially within the outer retina, which are common structural complications of retinovascular disease and the most common cause of vision loss in both DR and RVO. ${ }^{13}$ Thus, a better understanding of RPE pathobiology is instrumental to better understanding of retinovascular disease in general. Diabetics are known to have increased levels of serum homocysteine (Hcy), which correlates with the level of DR severity. ${ }^{14}$ Moreover, HHcy conditions disrupt the BRB in retinal endothelial cells and disrupt RPE cell structure and function. ${ }^{4,15}$ Thus, HHcy-treated ARPE-19 cells are a model for studying the molecular and cellular effects of the

CONTACT Mahavir Singh 2 mahavirsingh@louisville.edu (-) Eye and Vision Science Laboratory, Department of Physiology, University of Louisville School of Medicine, Louisville, KY 40202, USA

"These authors contributed equally to this work.

Color versions of one or more of the figures in the artide can be found online at www.tandfonline.com/icey.

- 2018 Taylor \& Francis Group, uC 


\title{
Canadian Journal of Physiology and Pharmacology
}

\author{
Home About Us Journals Books Compilations Open Access Authors Librarians Societies
}

$\underline{\text { Home }}>\underline{\text { Journals }}>\underline{\text { Canadian Journal of Physiology and Pharmacology }}>$ List of Issues $>$ Volume Q. Number ja

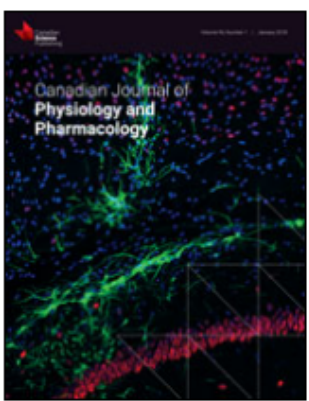

Browse the journal

" List of issues

we-First articles

"Just-IN articles

" Current issue

* Special issues

Most read articles

"Most cited articles

"Sample issue

* Author index

Restoration of skeletal muscle homeostasis by hydrogen sulfide during hyperhomocysteinemiamediated oxidative/ER-stress condition

A) $\underline{P D F}(1586 \mathrm{~K})$

A* PDF-Plus (1545 K)

Avisek Majumder; , Mahavir Singh, DVM, PhD, Akash K George, Suresh C. Tyagi ;

Published on the web 13 November 2018.

Received August 29, 2018.

Canadian Journal of Physiology and Pharmacology, https://doi.org/10.1139/cjpp-2018-0501

\section{ABSTRACT}

Elevated homocysteine (Hcy), i.e., hyperhomocysteinemia (HHcy) causes skeletal muscle myopathy. Among many cellular and metabolic alterations caused by $\mathrm{HHcy}$, oxidative and endoplasmic reticulum (ER)-stress are considered the major ones; however, the precise molecular mechanism(s) in this process is unclear. Nevertheless, there is no treatment option available to treat $\mathrm{HHcy}$-mediated muscle injury. Hydrogen sulfide $(\mathrm{H} 2 \mathrm{~S})$ is increasingly recognized as a potent anti-oxidant, anti-apoptotic/necrotic/pyroptotic, and anti-inflammatory compound and also has been shown to improve angiogenesis during ischemic injury. Patients with CBS mutation produce less $\mathrm{H} 2 \mathrm{~S}$, making them vulnerable to Hcy-mediated cellular damage. Many studies reported bidirectional regulation of ER-stress in apoptosis through JNK activation, and concomitant attenuation of cell proliferation and protein synthesis via PI3KJAKT axis. Whether $\mathrm{H} 2 \mathrm{~S}$ mitigates these detrimental effects of $\mathrm{HHcy}$ on muscle remains unexplored. In this review, we discuss molecular mechanisms of HHcy-mediated oxidative/ER-stress responses, apoptosis, angiogenesis, and atrophic changes in skeletal muscle and how $\mathrm{H} 2 \mathrm{~S}$ can restore skeletal muscle homeostasis during HHcy condition. This review also highlights the molecular mechanisms on how $\mathrm{H} 2 \mathrm{~S}$ could be developed as a clinically relevant therapeutic option for chronic conditions that are

\section{For authors}

" About the journal

* Open Access 


\section{Canadian Journal of Physiology and Pharmacology}

Home About Us Journals Books Compilations Open Access Authors Librarians Societies

$\underline{\text { Home }}>\underline{\text { Journals }}>$ Canadian Journal of Physiology and Pharmacology $>$ List of Issues $>$ Volume 0. Number ja, $>$ CircRNAs constitute an inherent gene-regulatc

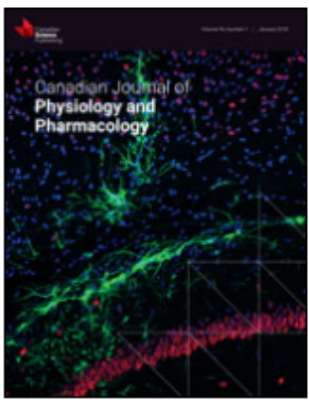

Browse the journal

List of issues

e-First articles

"Just-IN articles

* Current issue

"Special issues

Most read articles

" Most cited articles

"Sample issue

"Author index

For authors

"About the journal

* Open Access

\section{CircRNAs constitute an inherent gene- regulatory axis in the mammalian eye and brain}

A) $\underline{P D F}(1346 \mathrm{~K})$.

L* PDF-Plus (1349 K)

Akash K George, Kruyanshi Master, Avisek Majumder; , Rubens Petit

Homme, Anwesha Laha, Harpal Singh Sandhu, Suresh C. Tyagi ; , Mahavir Singh, DVM, PhD

Published on the web 16 November 2018.

Received August 30, 2018.

Canadian Journal of Physiology and Pharmacology, https://doi.org/10.1139/cjpp-2018-0505

\section{ABSTRACT}

CircRNAs are a new class of covalently closed transcripts that are produced via back-splicing. These molecules have been identified in organisms ranging from worm to plants. Research on circRNAs is an active area because of their diverse roles in health and in diseases. Their circularity makes them resistant to degradation thus they hold great promise as unique biomarkers. We believe that further work on their applications could help in developing them as "first-in-class" diagnostics, therapeutics, and prognostic targets for eye conditions. Many circRNAs play roles in transcriptional regulation by acting as miRNAs sponges. Since retina is an extension of brain and is part of CNS, we highlight the current state of circRNA biogenesis, properties and function and we review the crucial roles they play in the eye and brain. We also discuss their roles as miRNA sponges, regulation of genes or linear mRNAs, translation into micro-peptides/proteins, and responses to cellular stress. We posit that future advances will provide newer insights in the fields of RNA metabolism in general and diseases of the aging eye and brain. Furthermore, in keeping pace with the rapidly evolving discipline of RNA'omics'-centered metabolism and to achieve uniformity among researchers, we recently introduced the term "cromics" 
Avisek Majumder

PRESENT ADDRESS

1800 S $2^{\text {nd }}$ St., Apt A15

Louisville, KY-40204, USA
Email : avisek.majumder@louisville.edu avisek.biochem@gmail.com

Mobile : +1-573-200-5978

\section{Currently position: PhD student at Biochemistry and Molecular Genetics department (Completing on December $14^{\text {th }}, 2018$ ) \\ University of Louisville, Louisville, Kentucky 40202, USA}

\section{Academic Qualifications:}

a) Master of Science from West Bengal University of Technology (2008-2010) in Genetic Engineering

b) Bachelor of Science from Vidyasagar University (2005-2008) in Biotechnology (Academic Achievement: University $2^{\text {nd }}$ position in Bachelor's degree)

Research Experience: Molecular Biology and Biochemistry (1st Nov 2011 to now)

Current Employment: Graduate Research Assistant, Thesis title: Mechanism of hyperhomocysteinemia mediated skeletal muscle myopathy; Funding agency: NIH. Institution: University of Louisville. Guide: Suresh C Tyagi (Prof, UofL)

\section{List of Publications:}

1. Majumder A, Singh M, Behera J, et al. Hydrogen sulfide alleviates hyperhomocysteinemiamediated skeletal muscle atrophy via mitigation of oxidative and endoplasmic reticulum stress injury. Am J Physiol Cell Physiol. 2018; doi:10.1152/ajpcell.00147.2018

2. Majumder A, Singh M, George AK, et al. Hydrogen sulfide improves post ischemic neoangiogenesis in hindlimb of cystathionine- $\beta$-synthase mutant mice via PPAR- $\gamma /$ VEGF axis. Physiological Reports 2018; DOI: 10.14814/phy2.13858

3. Majumder A, Singh M, George AK, et al. Restoration of skeletal muscle homeostasis by hydrogen sulfide during hyperhomocysteinemia-mediated oxidative/ER-stress condition. Can J Physiol Pharmacol. 2018; doi: 10.1139/cjpp-2018-0501. 
4. Majumder A, Singh M, George AK, et al. Remote Ischemic Conditioning as a Cytoprotective Strategy in Vasculopathies during Hyperhomocysteinemia: An Emerging Research Perspective. Journal of Cellular Biochemistry 2018; DOI: 10.1002/jcb.27603

5. Homme RP, Singh M, Majumder A, et al. Remodeling of Retinal Architecture in Diabetic Retinopathy: Disruption of Ocular Physiology and Visual Functions by Inflammatory Gene Products and Pyroptosis. Front. Physiol. 2018; doi: 10.3389/fphys.2018.01268

6. Laha A, Majumder A, Singh M, Tyagi SC, et al. Connecting homocysteine and obesity through pyroptosis, gut microbiome, epigenetics, peroxisome proliferator-activator receptor $\gamma$ (PPAR $\gamma$ ) and zinc finger protein 407 (Zfp407); Can J Physiol Pharmacol. 2018; doi: 10.1139/cjpp-20180037

7. Singh M, George AK, Homme RP, Majumder A, et al. Circular RNAs profiling in the cystathionine- $\beta$-synthase mutant mouse reveals novel gene targets for hyperhomocysteinemia induced ocular disorders; Experimental Eye Research 2018, doi: 10.1016/j.exer.2018.05.026

8. George AK, Master K, Majumder A, et al. CircRNAs constitute an inherent gene-regulatory axis in the mammalian eye and brain, Can J Physiol Pharmacol. 2018; doi: 10.1139/cjpp2018-0505.

9. George AK, Singh M, Homme RP, Majumder A, et al. A hypothesis for treating inflammation and oxidative stress with hydrogen sulfide during age-related macular degeneration; Int $\mathbf{J}$ Ophthalmol 2018; doi: 10.18240/ijo.2018.05.26

10. Singh M, George AK, Homme RP, Majumder A, et al. Expression Analysis of the Circular RNA Molecules in the Human Retinal Cells Treated with Homocysteine. Current Eye Research. 2018 doi: 10.1080/02713683.2018.1542005.

11. Majumder A, Singh M, Tyagi SC, et al. Post-menopausal breast cancer: from estrogen to androgen receptor; Oncotarget 2017; doi.org/10.18632/oncotarget.22156

12. Majumder A, Behera J, Jeremic N, Tyagi SC, et al. Hypermethylation: Causes and Consequences in Skeletal Muscle Myopathy; Journal of Cellular Biochemistry 2017; DOI: 10.1002/jcb.25841.

13. Majumder A, Sheth F, Patel M, et al. Effect of PPAR- $\gamma 2$ Gene Pro12Ala Polymorphism (Rs1801282) and Vitamin D3 on Glucose Homeostasis in Type 2 Diabetic Subjects from Gujarat-India; Molecular Cytogenetics 2014, 7(Suppl 1): P37

14. Majumder A, Doshi B, Sheth F, et al. Association of Vitamin D3 levels with Glycemic Control in Type 2 Diabetic Subjects from Gujarati population-India; Molecular Cytogenetics 2014, 7(Suppl 1): P36

15. Shah A, Sheth F, Majumder A, et al. Effect of PPAR- $\gamma 2$ gene Pro12Ala and ADR- $\beta 3$ gene 
Trp64AArg polymorphism on glucose homeostasis in Type 2 diabetic subjects from GujaratIndia; Molecular Cytogenetics 2014, 7(Suppl 1): P101

16. Majumder A, Sheth J, Sheth F, et al. Effect of PPAR- $\gamma 2$ gene polymorphism (Pro12Ala) on HbA1C and its association with BMI in Type 2 diabetes subjects from Western India; Endocr Rev 2013; Vol. 34

\section{List of papers under way:}

1. Laha A, Majumder A, Singh M, et al. Regulation of Forkhead Box class O and Cell Death in Skeletal Muscle by Hydrogen Sulfide treatment during Hyperhomocysteinemia. Can J

Physiol Pharmacol. 2018; MS-ID: cjpp-2018-0496. [under review]

2. George AK, Homme RP, Majumder A, et al. Hydrogen sulfide intervention in cystathionine- $\beta$ synthase mutant mouse helps restore ocular homeostasis. Int J Ophthalmol 2018; MS ID: IJO-2018-0569. [under review]

3. Homme RP, Laha A, Majumder A, et al. Modulation of Inflammatory Pathway via inhibition of Nuclear Factor-Kappa Beta to Mitigate Vasculopathy in Diabetic Retinopathy. Int J Ophthalmol 2018; MS ID: IJO-2018-8814. [under review]

4. Master K, Majumder A, George AK, et al. Circular RNAs as the master genome regulators in the eye genome. Molecular Vision. [will be submitted soon]

5. George AK, Majumder A. Homme RP, et al. Genetics of Hyperhomocystenemia and ocular diseases. Ophthalmic Genetics. [will be submitted soon]

\section{Travel grant recommended:}

1. AHA predoctoral fellowship 2018 [submitted]

2. ASBMB travel award $\$ 1000$ for Annual Meeting of Experimental Biology 2018 (from ASBMB) held at San Diego, CA.

3. Early career forum travel grant, 2013: (from Endocrine Society, USA) $\$ 400$ \& complementary registration fees.

4. Department of Biotechnology; Ministry of Science and Technology, 2013: full travel award for attending ENDO 2013: The 95th Annual Meeting \& Expo. held at San Francisco, CA.

\section{$\underline{\text { Abstract Accepted in International Conference: }}$}


1. Evaluation of hydrogen sulfide $\left(\mathrm{H}_{2} \mathrm{~S}\right)$ effects on visual functions and pyroptosis in a mouse model of hyperhomocysteinemia. ARVO Annual Meeting Abstract Honolulu, Hawaii, April 29 - May 3, 2018:

2. Hyperhomocysteinemia-Mediated Endoplasmic Reticulum Stress in Skeletal Muscle Dysfunction via JNK/pro-inflammatory Pathway, FASEB Journal, Vol. 32, No. 1_supplement April 2018, Annual meeting of Experimental Biology-2018, San Diego, CA.

3. Hydrogen Sulfide Improves Hyperhomocysteinemia-Mediated Impairment of Angiogenesis in Skeletal Muscle; FASEB Journal, Vol. 32, No. 1_supplement, April 2018, Annual meeting of Experimental Biology-2018, San Diego, CA.

4. Ciglitazone Prevent Inflammation in Skeletal Muscle Induced by Hyperhomocysteinemia; FASEB Journal, Vol. 31, No. 1_supplement April 2017; Annual meeting of Experimental Biology-2017, Chicago, IL.

5. Role of Hydrogen Sulfide $\left(\mathrm{H}_{2} \mathrm{~S}\right)$ on Homocysteine Mediated Glutamate Excitotoxicity, Endoplasmic Reticulum Stress and Pyroptosis in Retina; FASEB Journal, Vol. 32, No. 1_supplement April 2018, Annual meeting of Experimental Biology-2018, San Diego, CA.

6. Effect of $P P A R-\gamma 2$ gene Pro12Ala polymorphism (rs1801282), vitamin $\mathrm{D}_{3}$ and $\mathrm{BMI}$ in type 2 diabetic subjects; its association with hemoglobin A1c level: a study on 902 subjects from Western India; ENDO 2014: The 96th Annual Meeting \& Expo. International conference to be held at Chicago, IL.

7. Effect of PPAR2 gene polymorphism (Pro12Ala) on HbA1C and its association with BMI in Type 2 diabetes subjects from Western India; ENDO 2013: The 95th Annual Meeting \& Expo. International conference held at San Francisco, CA from June 14-18, 2013.

8. Recommended for Young Scientist Award for present full paper entitled "Effect of PPAR- $\gamma 2$ Gene Pro12Ala Polymorphism (Rs1801282) and Vitamin D3 on Glucose Homeostasis in Type 2 Diabetic Subjects from Gujarat-India" 39th Annual Meeting of the Indian Society of Human Genetics; International seminar held at the Ahmedabad, India, from 22nd - 25th January 2014.

9. Antenatal screening of HbF levels show gender-biasness in haemoglobin disorders $(\mathrm{HbE} \beta, \beta)$ : A study in age-matched patients in Eastern Indian Population" International seminar on 10th Annual Conference of Indian Society for Prenatal Diagnosis \& Therapy held at Mumbai, India.

\section{PREVIOUS RESEARCH EXPERIANCES:}


Last Employed: Research Fellow at Institute of Human Genetics, Genetic Centre, India (2nd April 2012 to $31^{\text {st }}$ March 2014)

Project Title: "Effect of genetic variations in $P P A R \gamma-2$ and $A D R \beta-3$ gene in type 2 diabetic subjects of Gujarat, in relation to drug response"; Funding agency: Gujarat Institute of Chemical Technology (GICT). Institution: Foundation for Research in Human Genetics and Endocrinology, Institute of Human Genetics. Guide: Jayesh J Sheth, Ph.D., Director, Institute of Human Genetics (MS University affiliated)

\section{1) From $2^{\text {nd }}$ November 2011 to $28^{\text {th }}$ March, 2012:}

Project Title: "A Polymorphism in the Gene for $I G F-1$ and its Functional Properties and Risk for Type 2 Diabetes and Myocardial Infarction"

Institution: Molecular biology Dept, Galaxy Concepts Private Limited (ISO 9001-2008 certified), Kolkata, India. Guide: Dr. Prianka Pusp Research associate and faculty in Galaxy Concepts Private Limited.

Brief Description: There are many evidences that low levels of $I G F-1$ play a role in the pathogenesis of type 2 diabetes and cardiovascular diseases. I studied the role of a genetic polymorphism in the promoter region of the $I G F-1$ gene and measured anthropometric indices and other biochemical parameters along with genotyping. Conclusion: My study suggests that a genetically determined exposure to relatively low $I F G-1$ levels is associated with an increased risk for type 2 diabetes and myocardial infarction.

\section{2) From $1^{\text {st }}$ October 2009 to $30^{\text {th }}$ June, 2010:}

Project Title: "Study of IVS 1-5 G $\rightarrow$ C mutation by ARMS-PCR method in E $\beta$-thalassaemia patients to determine genotype-phenotype correlation". Institution: Institute of Genetic Engineering (Affiliated to WBUT), West Bengal, India. Guide: Dr. Sudipa Chakravarty, Vice Principal of Institute of Genetic Engineering.

Brief Description: I studied E $\beta$-thalassaemia patients with age ranging from 11 months to 50years. Studied Hemoglobin parameters (HbF, HbA0, HbA2) by HPLC method and CBC parameters (MCV, MCH, MCHC, HCT etc.) by Automated Cell Counter and genetic analysis was performed by ARMS-PCR for IVS $1 \rightarrow 5$ GC. Conclusion: a) Patients having IVS 1-5 G $\rightarrow$ C mutation are clinically less severe/ better i.e. most of these patients require either occasional or no blood transfusion. b) The Severity of the haemoglobinopathy disease process is related to genetically predetermined rate of either deficient globin chain production or complete cessation of its synthesis. 


\section{3) From $2^{\text {nd }}$ October 2008 to $30^{\text {th }}$ April, 2009:}

Project Title: "Phenotypic analysis of SNP variants of the alpha chain of Globin protein related to haemoglobinopathy". Institution: Institute of Genetic Engineering (Affiliated to WBUT), West Bengal, India. Guide: Dr. Amit Chakravarty, director of Institute of Genetic Engineering.

Brief Description: The PDB file of deoxy-hemoglobin was collected from RCSB-PDB database. The studies of SNPs were done by inducing previously collected mutations in deoxy hemoglobin structure by using Swiss-PDB Viewer software. Then the energy of the mutated structures was minimized using GROWMACS and RMSD values were calculated using VMD. Finally, a graph was plotted against RMSD values vs. type mutations. Conclusion: Non-polar to polar or polar to non-polar mutations are more severe than non-polar to non-polar or polar to polar mutations.

\section{Extra Qualifications (Certificates):}

- 3 months training in Assisted Reproductive Technology in human (IVF, ICSI, IUI).

- Training in Biotechnology techniques and its application.

- Workshop on Metabolic and Molecular Diagnosis- clinical \& laboratory approach.

- Certification Course in computer languages $(\mathrm{C}, \mathrm{C}++)$

- Technical Expertise - O.S. Platforms: WIN; LINUX; Mac; Scripting languages: HTML; M.S. office and other Software's.

\section{Seminars / Conference attained (Award \& Certificates):}

- Annual meeting of Experimental Biology-2018, San Diego, CA, USA.

- Annual meeting of Experimental Biology-2017, Chicago, IL, USA.

- The 95th Annual Meeting \& Expo Endocrine Society 2013, San Francisco, CA, USA.

- National Symposium on 10th Annual Conference of "Indian Society for Prenatal Diagnosis \& Therapy" in Mumbai, India.

- Diabetes \& its Complications: Search for Prevention \& Cure, National Seminar, Ahmedabad, India.

- Award \& Certificate for Best Project presentation on Post Genome impact from "1st Annual DNA Day contest” in Institute of Genetic Engineering (WBUT), West Bengal, India.

- $4^{\text {th }}$ World Congress on "Mild Approaches in Assisted Reproduction" arranged by International Society for Mild Approaches in Assisted Reproduction (ISMAAR), Kolkata, India. 